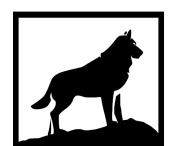

Michigan Technological

18 8 5 University
Michigan Technological University Digital Commons @ Michigan Tech

DEGRADABLE ZINC MATERIAL CHARACTERISTICS AND ITS INFLUENCE ON BIOCOMPATIBILITY IN AN IN-VIVO MURINE MODEL

Roger J. Guillory II

Michigan Technological University, rjguillo@mtu.edu

Copyright 2019 Roger J. Guillory II

Recommended Citation

Guillory II, Roger J., "DEGRADABLE ZINC MATERIAL CHARACTERISTICS AND ITS INFLUENCE ON BIOCOMPATIBILITY IN AN IN-VIVO MURINE MODEL", Open Access Dissertation, Michigan Technological University, 2019.

https://doi.org/10.37099/mtu.dc.etdr/967

Follow this and additional works at: https://digitalcommons.mtu.edu/etdr

Part of the Biomaterials Commons 


\title{
DEGRADABLE ZINC MATERIAL CHARACTERISTICS AND ITS INFLUENCE ON BIOCOMPATIBILITY IN AN IN-VIVO MURINE MODEL
}

\author{
By \\ Roger J. Guillory II \\ A DISSERTATION \\ Submitted in partial fulfillment of the requirements for the degree of \\ DOCTOR OF PHILOSOPHY \\ In Biomedical Engineering \\ MICHIGAN TECHNOLOGICAL UNIVERSITY \\ 2019 \\ (C) 2019 Roger J. Guillory
}


This dissertation has been approved in partial fulfillment of the requirements for the Degree of DOCTOR OF PHILOSOPHY in Biomedical Engineering.

Department of Biomedical Engineering

\author{
Dissertation Co-Advisor: Jeremy Goldman, $\mathrm{PhD}$ \\ Dissertation Co-Advisor: Jaroslaw Drelich, PhD \\ Committee Member: $\quad$ Feng Zhao, PhD \\ Committee Member: $\quad$ Megan Frost, PhD \\ Committee Member: $\quad$ Shu Q. Liu, PhD
}

Department Chair: $\quad$ Sean J. Kirkpatrick, PhD 
To my parents Roger J. Guillory Sr., \&

\section{Lillie D. Guillory,}

And my sisters

Deandrea C. Wright, \& Deangela P. Guillory 


\section{Table of Contents}

Preface

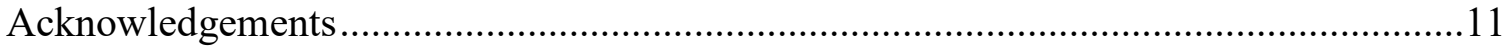

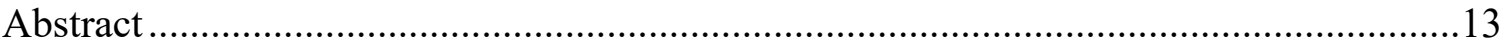

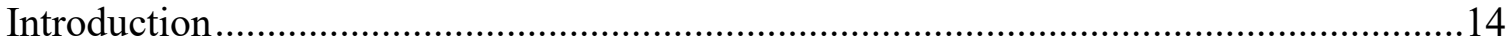

1.2 Heart disease and the state of the art ..........................................................

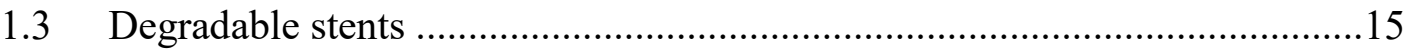

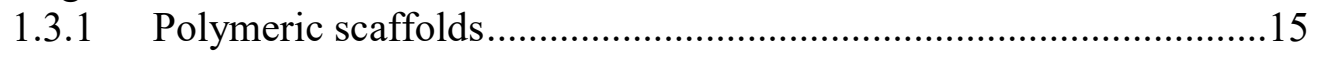

1.3.2 Degradable metals..........................................................................15

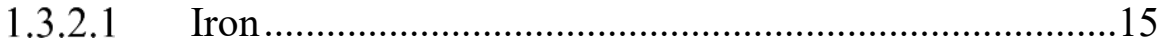

1.3.2.2 Magnesium...............................................................

1.3.2.3 Zinc .........................................................................

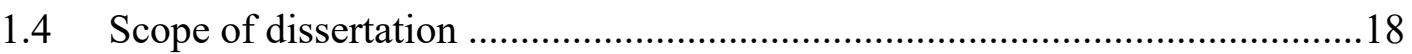

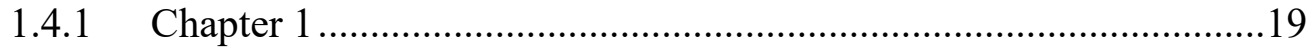

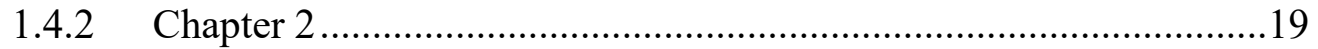

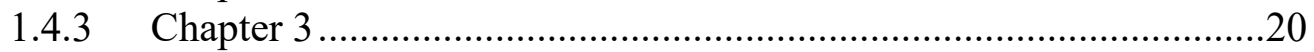

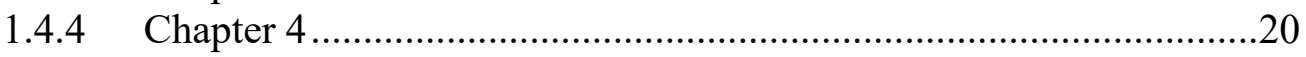

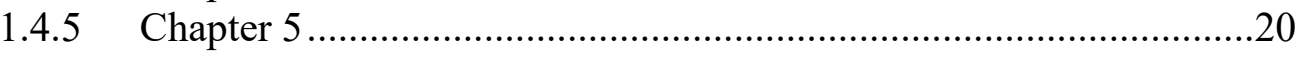

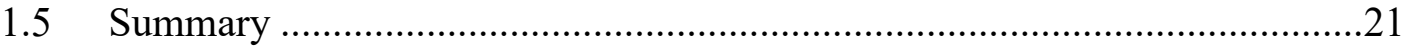

1 Understanding zinc material corrosion characteristics and the foreign body response

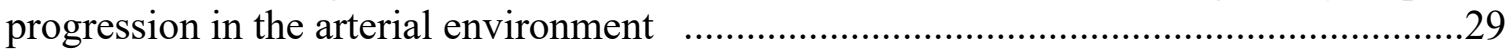

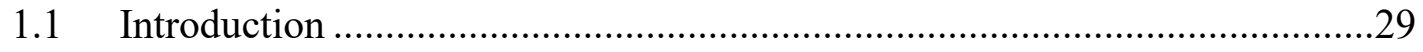

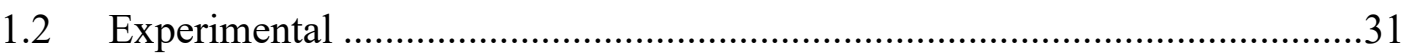

1.2.1 Materials and reagents ....................................................................

1.2.2 In vivo implantation and cryo-sectioning ..........................................33

1.2.3 SEM preparation and backscattered imaging ....................................34

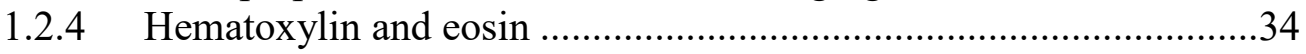

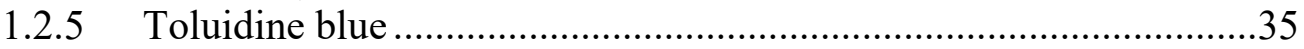

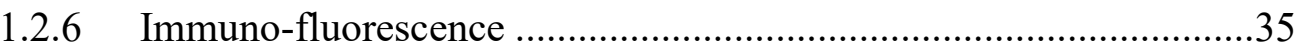

1.2.7 Immunofluorescent Quantification ..................................................36

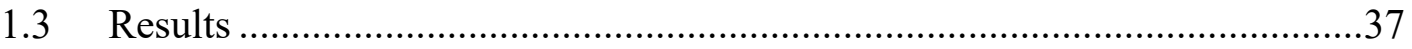

1.3.1 Backscattered imaging and cross sectional area reduction

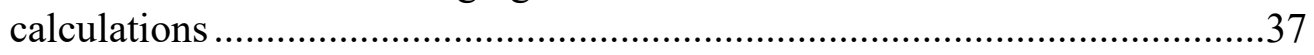

1.3.2 Hematoxylin and Eosin...................................................................

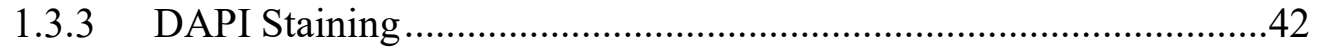

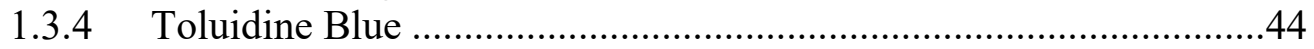

1.3.5 CD68,CD11b, and CD163 fluorescence ......................................46 


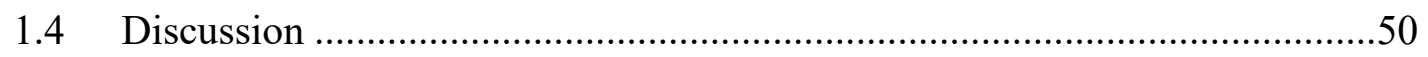

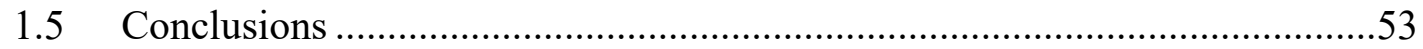

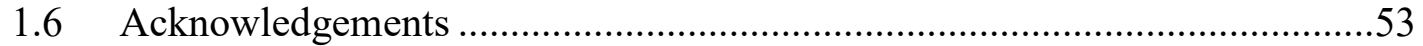

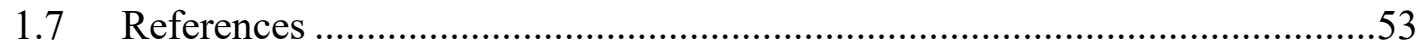

2 Development of novel in vivo methodology to screen degradable materials for

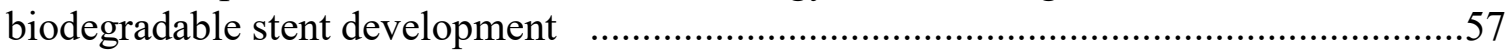

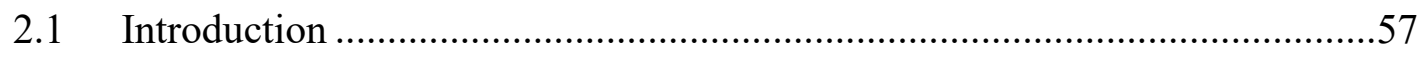

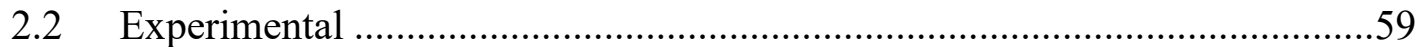

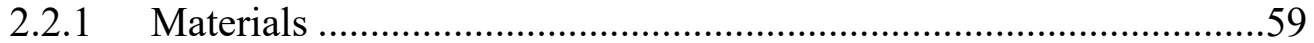

2.2.2 Surgical Implantation.....................................................................59

2.2.3 Implant removal and gross examination ............................................62

2.2.4 Tissue preparation and histological evaluation...................................62

2.2.4.1 Histological Staining and Morphometric Analysis..........64

2.2.4.2 Zn Accumulation in Organs............................................65

2.2.5 Statistical Analysis......................................................................66

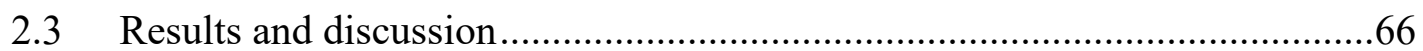

2.3.1.1 General histological presentation....................................67

2.3.1.2 Identification of optimal performance .............................70

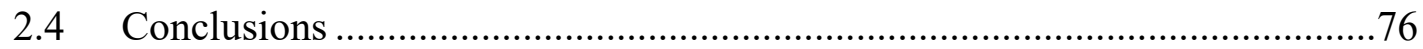

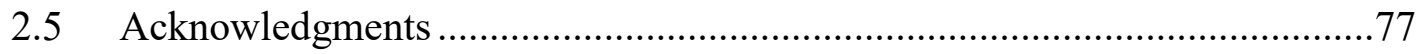

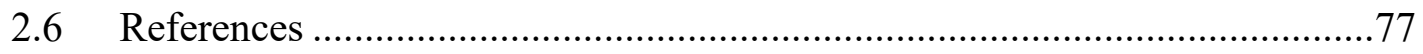

3 Contributions of the surface characteristics of degradable zinc implants to the neointimal response and subsequent biocompatibility ..................................................79

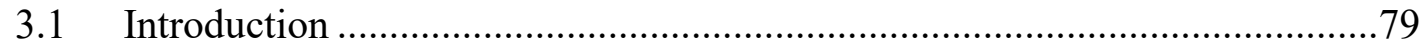

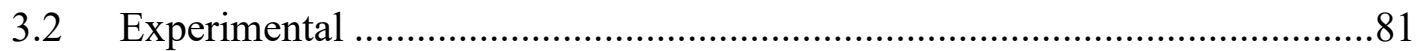

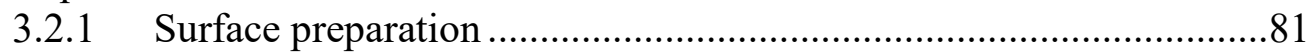

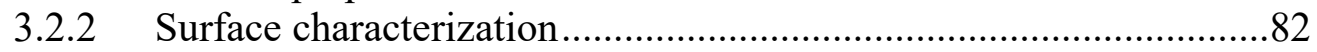

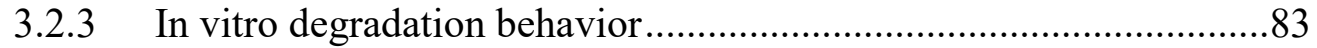

3.2.4 In vivo wire implantation............................................................... 84

3.2.5 Tissue preparation, histological, and morphometric analysis ............84

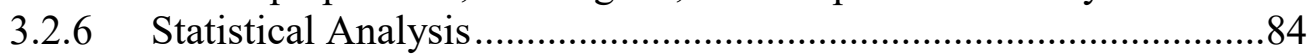

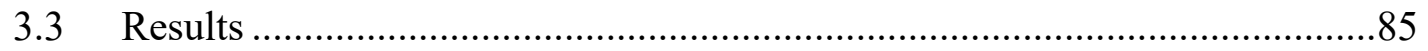

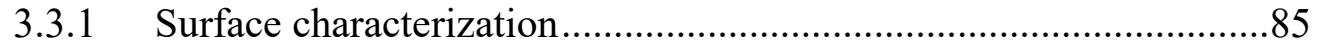

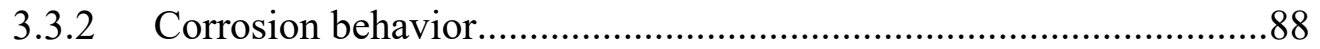

3.3.3 Histomorphometry and biocompatibility ……………......................94

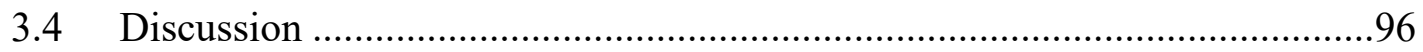




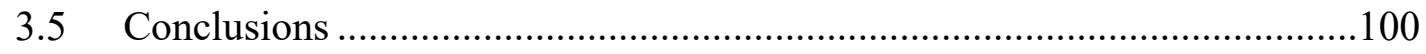

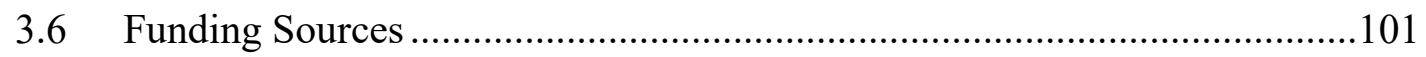

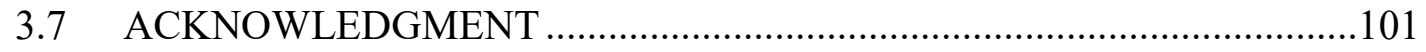

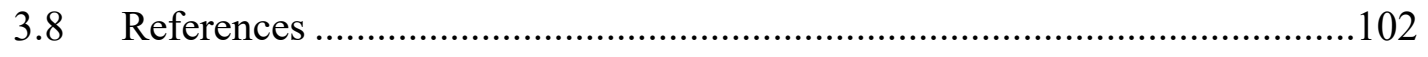

4 In-Vivo Biocompatibility of Next Generation Zn-Ag Based Stent Materials .......107

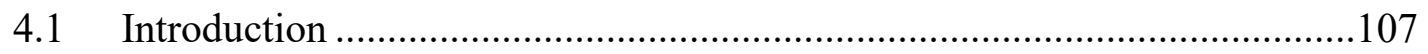

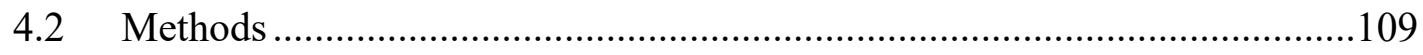

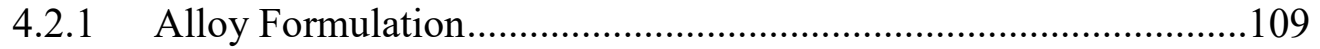

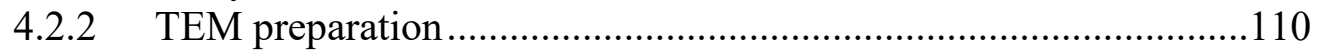

4.2.3 Implantation and collection...........................................................110

4.2.4 Statistical analysis ......................................................................111

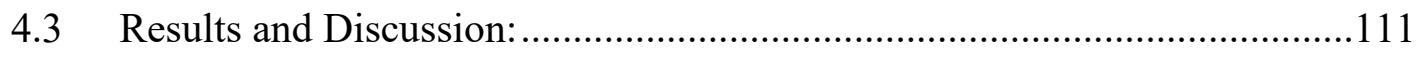

4.3.1 Microstructural Characterization ..................................................111

4.3.2 General Histologic Presentation ...................................................113

4.3.3 Effects of alloying......................................................................... 115

4.3.4 Effects of nano-precipitate phases ………………........................118

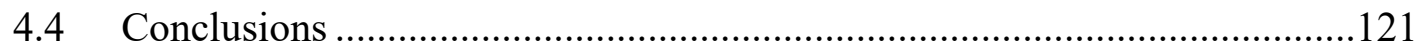

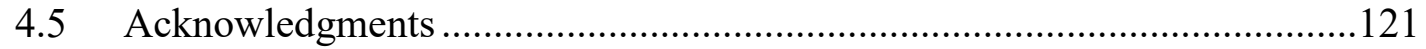

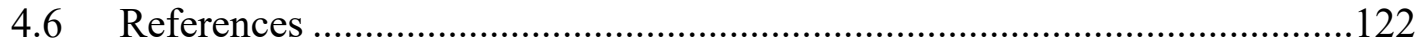

5 Zinc Based Materials Regulate the Vascular Biologic Response to Implants by Releasing Therapeutic Transition Metal Ions ............................................................125

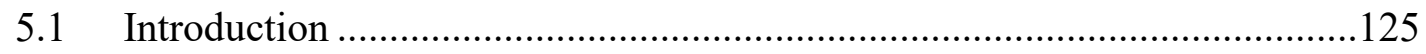

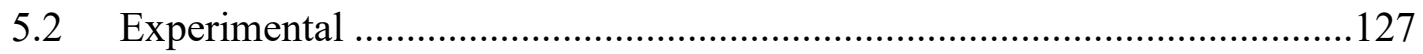

5.2.1 Materials ………………………………..............................127

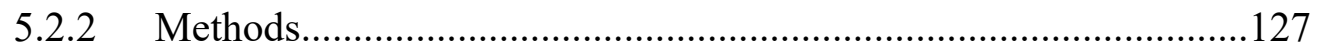

5.2.2.1 In vivo Implantation...................................................127

5.2.2.2 General Histology, immunofluorescence and in-situ

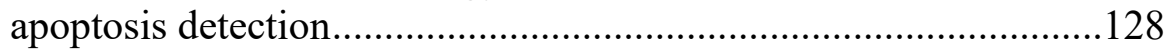

5.2.2.3 Ex-vivo arterial ring tissue culture.................................128

5.2.2.4 Multi-caspase activity assay ………………….............129

5.2.2.5 Confocal microscopy ..................................................129

5.2.2.6 Corrosion of alloys and ICP-OES analysis ....................130

5.2.2.7 Statistical Methods ........................................................130

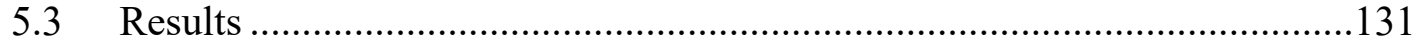

5.3.1 Morphometric presentation ............................................................131

5.3.2 TUNEL labeling of NI and $\alpha$-SM / active caspase-3 presence ......132

5.3.3 Dose dependent caspase $-3,-8$, and -9 activation by $\mathrm{Zn}$.................134 
5.3.4 Effect of transition metal ions on caspase activity.......................134

5.3.5 Addition of $\mathrm{Cu}$ to bulk zinc materials..........................................136

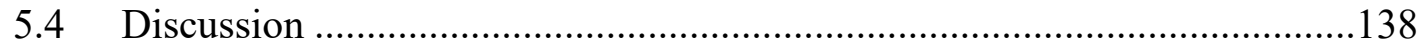

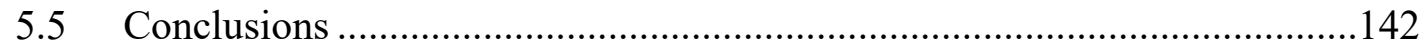

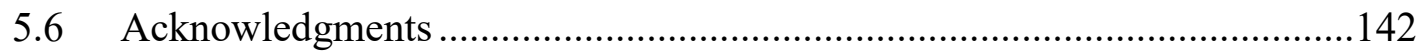

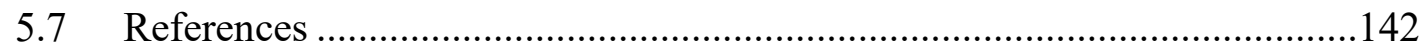

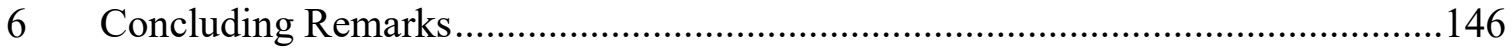

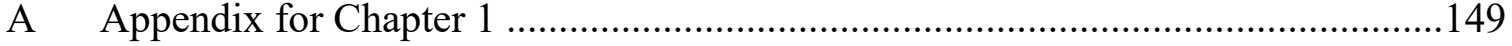

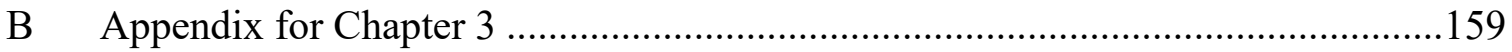

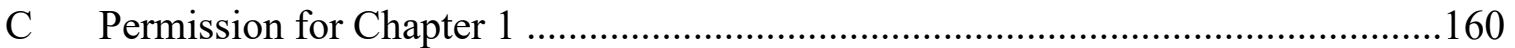

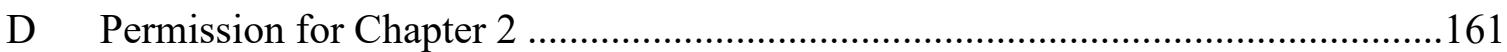

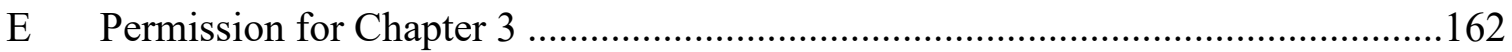




\section{Preface}

This dissertation is the presented works of 5 interdependent chapters, three of which are published in journals and two in preparation for publication. The contributions of each body of work will be explained rigorously in this section. In all works, Dr. Jeremy Goldman and Dr. Jaroslaw Drelich provided guidance, editing, and final approval of the manuscript. Dr. Jeremy Goldman conducted most surgical in vivo implantations.

The first chapter investigates first-generation zinc materials and relates host inflammatory reaction to corrosion characteristics. It is under the full citation:

1. Guillory, Roger J., Patrick K. Bowen, Sean P. Hopkins, Emily R. Shearier, Elisha J. Earley, Amani A. Gillette, Eli Aghion, Martin Bocks, Jaroslaw W. Drelich, and Jeremy Goldman. "Corrosion characteristics dictate the long-term inflammatory profile of degradable zinc arterial implants." ACS Biomaterials Science \& Engineering 2, no. 12 (2016): 2355-2364.

The experimental design, execution, writing, and data analysis was mostly performed by Roger J. Guillory II. Dr. Patrick Bowen contributed to SEM and backscattered electron imaging of the implants, and writing/editing sections of the manuscript. Dr. Sean P. Hopkins contributed to data analysis of the macrophage staining and writing. The other co-authors contributed to histological staining procedures and final review of the manuscript.

The second chapter discusses the development of a novel preclinical model to screen degradable metal candidates for cardiovascular stents. The full citation is provided below:

2. Guillory, Roger J., Alexander A. Oliver, Emma K. Davis, Elisha J. Earley, Jaroslaw W. Drelich, and Jeremy Goldman. "Preclinical In Vivo Evaluation and Screening of ZincBased Degradable Metals for Endovascular Stents." JOM 71, no. 4 (2019): 1436-1446.

The design, execution and data analysis were primarily performed by Roger J. Guillory. Roger J. Guillory wrote the entire manuscript, and all authors contributed to revisions prior to final publication. Emma k. Davis created one of the 7 figures in the manuscript, and Alexander Oliver and Elisha Earley helped with histological sectioning and staining. 
The third chapter investigates the relationship between the surface of degradable zinc materials and the subsequent vascular response, using the model described in the second chapter. The full citation is provided below:

3. Guillory II, Roger John, Malgorzata Sikora-Jasinska, Jaroslaw W. Drelich, and Jeremy Goldman. "In Vitro Corrosion and In Vivo Response to Zinc Implants with Electropolished and Anodized Surfaces." ACS applied materials \& interfaces(2019).

Roger J. Guillory designed the experiments, performed all histology and sectioning, and processed all in-vivo data. Dr. Malgortza Sikora-Jasinska performed all in vitro corrosion experiments, with both authors developing the surface treatments used throughout the study. XPS was performed by Dr. Timothy Leftwhich, with sample preparation performed by Roger J. Guillory and Dr. Malgorzata Sikora-Jasinska. Dr. Malgorzata Sikora-Jasinska participated in writing sections of the manuscript and prepared 2 figures fully and one figure jointly. Roger J. Guillory wrote most of the manuscript and prepared 2 figures fully, and one figure jointly.

The fourth chapter describes the newest generation of zinc-based materials developed by our research group. This chapter is in preparation to be submitted to an academic journal. Roger J. Guillory designed the experiments, performed data analysis and wrote the entire manuscript. Dr. Ehsan Mostaed developed the novel material and produced wires from castings. Roger J. Guillory constructed the figures, with the exception of the TEM figure produced by Dr. Ali Mostaed, which was made by Dr. Ehsan Mostaed. The other coauthors participated in histological staining and data collection.

The last chapter investigates an exciting phenomenon seen at the interface of zinc-based materials in the vascular environment. This work is in preparation to be submitted to an academic journal. Roger J. Guillory designed the experiments and performed histological processing, enzymatic assays, data analysis, constructed figures, and wrote the entire manuscript. Alexander Oliver participated in histological data collection and analysis. Timothy Kolesar and Lea Morath performed enzyme activity assays and histological 
staining. Dr. Malgorzata-Sikora Jasinsaka performed ICP analysis from in vitro corrosion studies. 


\section{Acknowledgements}

I would first like to thank to two advisors, Dr. Jeremy Goldman and Dr. Jarek Drelich. My journey into the scientific arena was solely facilitated by these two. For nearly 8 years, they have consistently supported me, and have had a genuine interest and desire for my success as a scientist. For that, I am grateful.

My dissertation committee, Dr. Feng Zhao, Dr. Megan Frost, and Dr. Shu Q. Liu, have provided me with guidance throughout my journey as a Ph.D. student. The wisdom provided by these exceptional scientist and engineers has helped me develop, and reach my full potential here at Michigan Tech.

To Amani Gillette, thank you for your patience and support throughout this journey. I would like to thank Dr. Patrick Bowen and Dr. Emily Shearier for their guidance and mentorship throughout my training and for their unwavering support.

My dissertation work largely benefitted from the direct help and support from co-authors and fellow colleagues. In no particular order, Alexander Oliver, Maria P. Kwesigia, Jeffery M. Brookins, Morteza Shaker, Avishan Arab-Shomali, Elisha Earley, Adam Drelich, Dr. Shan Zhao, Lea Morath, Dr. Sean P. Hopkins, Dr. Connor McCarthy, Katie Flonn, Emma K. Davis, Kevin Sunderland, Pegah Forooshani, Dr. Timothy Kolesar, Dr. Patrick Bowen, Dr. Ehsan Mostaed, Dr. Emily Shearier, Dr. Malgorzata- Sikora Janinska, Jennifer Eikenberry, Courtney Perreault,

A special thanks to ACMAL, Owen Mills, Daniel Seguin, Dr. Timothy Leftwich for assistance with material characterization. To our Biomedical Engineering Department staff, Corey Dompier and Stacey Sedar for all of their hard work and efforts to make graduate school administratively easy.

There are many people, while not contributing to my dissertation directly, that have had substantial influence on me throughout my years here at Michigan Tech. Dr. Tarun Dam, 
Dr. Melissa Baird, Susan Libeau, Darnisha Slade Kellie Rafaelli, Dr. Pushpalatha Murthy, Karmen Markham, 


\section{Abstract}

Biodegradable stents based on zinc have been under development since their introduction in 2013. While metallic zinc is highly ductile, it unfortunately lacks the mechanical strength required for arterial stents. This has led to the development of an abundance of novel zinc-based materials, with the aim of improving the mechanical strength without sacrificing too much ductility. Although these materials are intended to function and slowly degrade within an artery, most zinc-based materials have been developed without deep consideration for their biological effects.

The present work explores the biological effects elicited by zinc-based materials implanted within the arterial system. The biological effects of degradable arterial implants were characterized in terms of quantifiable metrics, including neointimal area, implant to lumen thickness, and base neointimal length. These metrics were used to clarify relationships between material characteristics, including surface oxide film stability, elemental composition, and microstructure, with biological responses. The metrics were also used to compare materials in terms of their biocompatibility. In addition to evaluating biocompatibility, beneficial elements identified by these approaches can be further investigated for their therapeutic value, since all the elements in the implant will be released due to implant degradation. The combined work makes it possible to screen materials in terms of their biocompatibility and provides fundamental insights that impact the metallurgical design of materials. 


\section{Introduction}

\subsection{Heart disease and the state of the art}

Heart disease poses an extreme health and monetary risk for Americans. According to the most recent update by the American Heart Association (2017), 92.1 million US adults have at least one type of cardiovascular disease. The average annual cost from heart disease to Americans in 2013 was 199.6 billion USD. Additionally, myocardial infarction and coronary heart disease were 2 of the 10 most expensive hospital diagnoses[1]. The most predominant underlying pathology of myocardial infarction is atherosclerosis. Atherosclerosis is a complex, progressive disease characterized by events such as lipid deposition, intra-arterial plaque formation and buildup, inflammatory mediation and subsequent thrombogenic events emanating from unstable ruptured plaques, all of which contribute to luminal obstruction[2-4].

Presently, the gold standard for treatment of atherosclerosis-induced arterial stenosis is balloon catheter stenting. These metal scaffolds increase the cross-sectional area of the arterial lumen, enhancing downstream blood flow to vital organs (e.g. heart or brain). While the early term effects of stenting diseased arteries are generally positive, the permanent residence of the metal scaffold within the vasculature elicits chronic negative effects. These deleterious effects can include smooth muscle cell intimal hyperplasia due to persistent inflammation[4], stent thrombosis[5], and de novo neoartherosclerosis[2, 6-8].

Over the past 15 years, advances within the interventional cardiology sector have aimed to mitigate early negative outcomes with paclitaxel and sirolimus polymer loaded drug eluting stents[9] (DES), which successfully reduce restenosis rates compared to bare metal stents[10]. Although first generation DESs were effective initially, delayed healing/ endothelialization and an increased risk of very late stent thrombosis have plagued first generation DESs[11]. Second generation everolimus DESs are superior to first generation DESs[10], yet still succumb to 
neoatherosclerosis after a long term residence, which appears to be an end term failure mode of any implanted stent regardless of drug elution[7].

\subsection{Degradable stents}

\subsubsection{Polymeric scaffolds}

Inadequate mitigation of negative long-term permanent stent outcomes has generated an intense interest for exploring alternative solutions. The most promising alternative approach is to develop a stent that is completely and safely absorbed over time by local vascular tissue. This concept was pioneered by Tami et al, who deployed 25 poly-L-lactic acid stents in 19 lesions across 15 patients with a 6 month follow up time. The study concluded that no significant progressive intimal hyperplasia or lumen loss over 3 and 6 months were provoked by the degradable PLLA based stents[12]. A ten year follow up of the Igaki- Tami stents proved the efficacy of a degradable stent, with mean complete degradation of around 3 years[13]. The 2014 Absorb trials of fully degradable PLLA- everolimus eluting stents showcased the efficacy of drug eluting degradable stents[14]. Although the success of degradable polymeric materials has been clearly shown, this class of materials cannot feasibly achieve the mechanical benchmarks required for arterial stents. This inherent limitation restricts polymeric materials to treating simple lesions of short length. Additionally, the lower radial strength of polymers increases the chances of acute stent recoil and strut malapposition and necessitates an enlarged polymer strut thickness. The excessive strut size and surface area increases arterial injury and thrombogenesis. These serious limitations argue for a degradable metallic alternative.

\subsubsection{Degradable metals}

\subsubsection{Iron}

Peuster and others were some of the first pioneers to investigate iron as a potential degradable metallic stent material [15]. They investigated the efficacy of 16 iron stents in 
the descending aorta of New Zealand white rabbits. While these stents performed well with no apparent toxicity and inflammatory response, they did not investigate the ability of the stent to fully corrode. A long term (12 month) follow up study by the authors determined that there was a need to increase the degradation rate of iron materials, as most of the stent remained after the 1 year follow up period [16]. Many investigations after these landmark studies focused on modifications of the bulk iron material to increase the degradation rate [17-25]. More recent absorbable metal candidates have overtaken the use of iron as a base material.

\subsubsection{Magnesium}

The most obvious degradable metal candidate, and perhaps the most successful have been materials with a base composition of magnesium. An early study by Heublein and colleagues demonstrated the feasibility of using a non commercial magnesium based alloy to serve as a degradable scaffold, in swine[26]. This initial success for magnesium generated excitement within the degradable metals field, prompting implantations of fully degradable magnesium stents in newborns to treat congenital heart defects, with some degree of success[27, 28]. Unfortunately, the corrosion of magnesium suffers from excessive degradation rates, which is the opposite problem of iron-based materials. With corrosion rates between $19-44 \mathrm{mg} / \mathrm{cm}^{2} /$ day, it was estimated that a $5 \mathrm{~g}$ magnesium implant would lose functionality in 10-20 days, and be completely dissolved within 20-45 days[29]. Magnesium is also known to generate hydrogen gas through the following reaction;

$$
\mathrm{Mg}+2 \mathrm{H}_{2} \mathrm{O} \rightleftarrows \mathrm{Mg}^{2+}+\mathrm{H}_{2}+2 \mathrm{OH}^{-}
$$

Since magnesium degradation generates around $1 \mathrm{~L}$ of $\mathrm{H}_{2}$ per gram of pure material, processing and alloying approaches have received an intense focus from engineers intending to slow the degradation rate of magnesium. A lower degradation rate would be beneficial from an arterial healing standpoint and serve to reduce the rate of potentially 
damaging hydrogen gas evolution [30-32]. Although these challenges have precluded the translation of degradable magnesium into safe and effective arterial stents, magnesium still remains the most successful degradable metal material in terms of commercialization progression. A number of clinical trials have demonstrated the efficacy of metal-based degradable stents, including the DREAMS 2G (MAGMARIS) BIOSOLVE II and BIOSOLVE III $[33,34]$. Although there has been some success with Mg based scaffolds, problems include mechanical properties that do not yet approach that of conventional metal stents, yielding large strut sizes[35]. Another critical limitation of Mg based stents is the need for a drug eluting coating, which is a known contributor to late term stent thrombosis and delayed endothelialization[36]. Finally, despite extensive metallurgical modifications reported in the literature, $\mathrm{Mg}$ stents still degrade too rapidly ( $<12$ months) to support the proper healing of diseased vasculature[35].

\subsubsection{Zinc}

Bowen and colleagues introduced zinc in 2013 as a promising degradable metal candidate for coronary stenting[37]. With a penetration rate near the proposed benchmark of 20 $\mu \mathrm{m} /$ year, along with compact corrosion products, zinc materials quickly became a favored candidate for degradable metallic stents[38]. As with the other two degradable metal candidates, the potential upsides come with substantial limitations.

Pure zinc does not possess adequate mechanical properties for stenting, with a tensile strength around $120 \mathrm{MPa}$, although it has a rather pronounced elongation to failure of $60 \%-80 \%[38]$. Stenting materials should possess mechanical properties that are approximated in the table below. 
Table 1. Mechanical characteristics of Zn compared to stent material benchmark values

\begin{tabular}{|l|l|l|l|l|}
\hline \multirow{4}{*}{ Metal } & \multicolumn{3}{|l|}{ Mechanical Properties } & \multirow{2}{*}{ Grain } \\
\cline { 2 - 5 } & $\begin{array}{l}\text { Yield } \\
\text { Strength } \\
{[\mathbf{M P a}]}\end{array}$ & $\begin{array}{l}\text { Tensile } \\
\text { Strength } \\
{[\mathrm{MPa}]}\end{array}$ & $\begin{array}{l}\text { Elongation } \\
\text { to Failure } \\
{[\%]}\end{array}$ & $\begin{array}{l}\text { Size } \\
{[\boldsymbol{\mu} \mathbf{m}]}\end{array}$ \\
\hline $\begin{array}{l}\text { Benchmark } \\
\text { Value }\end{array}$ & $>200$ & $>300$ & $>15$ & $<30$ \\
\hline $\begin{array}{l}\text { Pure Zn } \\
\text { (reference) }\end{array}$ & $<80$ & $<120$ & $60-80$ & $>50-$ \\
\hline
\end{tabular}

With the introduction of zinc in 2013, questions surfaced regarding the biocompatibility of the metal. Bowen et. al. demonstrated the exceptional biocompatibility of pure zinc within the abdominal aorta of rats[39], using a wire implantation model developed by Pierson et. al. in 2012[40]. Zinc provoked relatively small neointima formations, with an notable gradient of smooth muscle cells receding towards the implant[39]. With the introduction of zinc materials and their optimal biocompatibility, a new wave of material development from metallurgists began to improve the mechanical properties through elemental additions and advanced processing [41-55].

\subsection{Scope of dissertation}

The excitement generated in the field of degradable $\mathrm{Zn}$ based stents led to a bottleneck of novel materials that required biocompatibility evaluations. The in vivo evaluation of degradable materials required a shift in the framework of modern biomaterials development and testing. Our group at Michigan Tech first developed an in-vivo "wire model" to simplify the characterization of the host response to degradable materials 
intended for use as cardiovascular stents[40]. This model provides a unique platform to study degradable materials in a fashion that allows detailed examination of the tissue material interaction. In order to develop successful zinc-based materials, a thorough understanding of the material-arterial cell interactions and the resulting biologic response is essential. We outline these new approaches and investigations in the chapters of this dissertation.

\subsubsection{Chapter 1}

This chapter investigates multiple zinc materials in an arterial wall implant model. The wall implant model was chosen initially as a simplified environment relative to the luminal space due to the avoidance of blood interaction. This work begins to clarify the relationship between material corrosion character and the ensuing host response. Classically, the foreign body response is a progressive reaction to an implanted biomaterial that eventually leads to fibrotic encapsulation. This classic understanding of the biological response to implanted materials, although widely accepted, must be adapted to incorporate the dynamic and complex biointeractive properties of degradable materials. Understanding the contribution of corrosion products and behavior to the vascular response and overall biocompatibility is of critical importance when designing biodegradable materials.

\subsubsection{Chapter 2}

Alloying of degradable zinc materials has become a standard approach to improve the mechanical properties. The primary evaluation of materials is a straightforward mechanical testing to compare novel materials to stent material benchmark values. When biocompatibility is evaluated, the primary start point is an insult of corrosion media supernatant extract to 2-D cultured cells. This approach, while useful for understanding basic toxicity, is not appropriate to clarify the in vivo performance of degradable metals. To clarify the biocompatibility of a degradable material, a realistic application environment is an essential start point. Here we develop a quantitative, engineering based 
approach to evaluate the performance of degradable materials in an in-vivo system. This approach, while possessing both strengths and weaknesses, can nevertheless help identify the best performing materials using a low cost, physiologically relevant animal model.

\subsubsection{Chapter 3}

A critical component of material design, particularly in metals that will eventually degrade, are the surface features. Long-term material corrosion characteristics of zinc materials can be directed by the material's initial surface conditions. Since the potential exists for the control of surface properties to make possible the "tunability" of corrosion dynamics, the relationship of surface characteristics to the vascular response must be clarified. This chapter describes an investigation to relate varied surface features of degradable zinc-based implants to the vascular response.

\subsubsection{Chapter 4}

Our group at Michigan tech has steadily developed a suite of degradable metal candidates for cardiovascular stents. Previously, it has been challenging to relate differences in neointimal character to specific modifications of materials. This chapter evaluates a new generation of degradable zinc alloys and provides insight into the relationship between elemental compositions and neointimal formation. Understanding the interplay between metallic elemental additions and vascular responses is critical to designing top performing alloys. In parallel, we have also discovered that changes to the microstructure affect neointimal responses. These two components of alloy design not only alter the mechanical properties of materials, but also critically impact biological outcomes.

\subsubsection{Chapter 5}

To date, we have examined zinc materials from a performance standpoint in order to select the best performing materials for continuation in the stent development pipeline. While this approach is useful from an engineering and commercialization standpoint, there is tremendous value in understanding the basic interactions between the material 
and arterial cells. Recently, Bowen and colleagues described an exciting phenomenon of neointimal smooth muscle cells organizing peripherally from the degradable zinc implant and avoiding the interface. In this contribution, the neointima of pure zinc materials is probed for smooth muscle cell content and compared to a non-degrading control. Molecules involved with the regulation and initiation of apoptosis are monitored. A material that can exert an anti-proliferative/pro-apoptotic effect on the migratory smooth muscle cells is ideal within the realm of vascular materials. Current generation stents require a drug eluting polymer coating to prevent excessive neointimal growth and limit harmful outcomes. Here, we find that the byproducts of zinc implant degradation may impart a suppressive effect against vascular smooth muscle cells and anti-inflammatory pro-healing effects on endothelial cells. This paradigm shift that emerges with degradable zinc materials within the vasculature may shift the way scientists think about degradation as a whole; not just as a peripheral feature of the material, but as central means of imparting continuous therapy to the treated vessel for the entire lifetime of the implant.

\subsection{Summary}

This dissertation provides new insights into the biocompatibility of degradable zinc implants for use in the cardiovascular system. Chapter 1 provides a rigorous and detailed histopathological analysis of zinc and zinc alloys in an in vivo implant model. The methodology used allows for a multifaceted approach to understanding the dynamic foreign body response to degrading materials. From this work, we are able to determine that the inflammatory response not only depends on the elemental additions, but manifests primarily from the inherent corrosion characteristics dictated by the elemental additions. These results demonstrate that tissue reactions towards degrading zinc materials can be regulated by controlling corrosion behavior.

With this newfound knowledge, a natural hypothesis arises that different degradable zinc materials (ie. Zn-Li vs. Zn-Mg) with different inherent corrosion properties will evoke different responses in the vascular system. To test this hypothesis, an analysis framework with appropriate design and outcome metrics needs to be established. Chapter 2 
accomplishes this by developing a reliable method for the quantification of biocompatibility metrics within the rodent aorta. This standardized methodology makes it possible to compare materials in terms of biocompatibility and relate the differences in biocompatibility of materials to material characteristics. By using histomorphometry parameters that are similar to those used when evaluating stents in the clinic, the results from the characterization of neointimal responses to wire implants can be compared to stents that are in clinical use.

Since it is apparent that corrosion characteristics affect neointimal growth and development, variations in the initiation of corrosion would be expected to dramatically alter the vascular response. In chapter 3 we vary the surface oxide film character of pure $\mathrm{Zn}$ substrates to investigate the effect of surface oxide film properties on early term neointimal responses. Interestingly, standard surface treatments used for permanent metallic materials may not be optimal for zinc implants. This is due to the initiation of harmful corrosion events that exacerbate neointimal progression when using zinc implants with electropolished surfaces.

Chapter 4 probes deeper into the neointimal responses of a wide variety of new generation alloys, using the established framework presented in Chapter 2. Complex alloy systems (elemental additions $>3$ ) provide unique changes at a microstructural level, which are found to directly impact the neointimal response/ performance of the material. Understanding the relationship between the microstructure and neointimal response provides predictive power that aids in the design of future materials.

Even though this body of work rigorously demonstrates that bulk corrosion, surface characteristics, and microstructural changes in $\mathrm{Zn}$ materials impact biocompatibility, the role of the corrosion byproducts in the biocompatibility of the implant remains a critical open question. Chapter 5 probes the cellular and protein content in the neointimal tissue surrounding $\mathrm{Zn}$ based implants. The results of the analyses demonstrate that ionic $\mathrm{Zn}$ initiates apoptotic death in vascular smooth muscle cells. Importantly, this chapter shows that metallic zinc may serve not only as structural support for the vessel, but its release in 
an ionic form profoundly affects neointimal cells through its interaction with proteinmediated cell signaling pathways.

Together, this body of work evaluates the impact of zinc-based materials as potential cardiovascular stents on biocompatibility by integrating a large spectrum of fields ranging from materials science, physiology, and cell and molecular biology. During the course of this multifaceted investigation, novel approaches are developed and material properties are related to neointimal growth and device performance.

[1] W.G. MEMBERS, E.J. Benjamin, M.J. Blaha, S.E. Chiuve, M. Cushman, S.R. Das, R. Deo, S.D. de Ferranti, J. Floyd, M. Fornage, Heart disease and stroke statistics - 2017 update: a report from the American Heart Association, Circulation 135(10) (2017) e146.

[2] C. Weber, H. Noels, Atherosclerosis: current pathogenesis and therapeutic options, Nature medicine 17(11) (2011) 1410.

[3] R. Kornowski, M.K. Hong, F.O. Tio, O. Bramwell, H. Wu, M.B. Leon, In-stent restenosis: contributions of inflammatory responses and arterial injury to neointimal hyperplasia, Journal of the American College of Cardiology 31(1) (1998) 224-230.

[4] P. Libby, Inflammation in atherosclerosis, Arteriosclerosis, thrombosis, and vascular biology 32(9) (2012) 2045-2051.

[5] I. Moussa, C. Di Mario, B. Reimers, T. Akiyama, J. Tobis, A. Colombo, Subacute stent thrombosis in the era of intravascular ultrasound-guided coronary stenting without anticoagulation: frequency, predictors and clinical outcome, Journal of the American College of Cardiology 29(1) (1997) 6-12.

[6] F. Otsuka, R.A. Byrne, K. Yahagi, H. Mori, E. Ladich, D.R. Fowler, R. Kutys, E. Xhepa, A. Kastrati, R. Virmani, Neoatherosclerosis: overview of histopathologic findings and implications for intravascular imaging assessment, European heart journal 36(32) (2015) 2147-2159.

[7] S.-J. Park, S.-J. Kang, R. Virmani, M. Nakano, Y. Ueda, In-stent neoatherosclerosis: a final common pathway of late stent failure, Journal of the American College of Cardiology 59(23) (2012) 2051-2057. 
[8] K. Yamaji, K. Inoue, T. Nakahashi, M. Noguchi, T. Domei, M. Hyodo, Y. Soga, S. Shirai, K. Ando, K. Kondo, Bare metal stent thrombosis and in-stent neoatherosclerosis, Circulation: Cardiovascular Interventions 5(1) (2012) 47-54.

[9] J.W. Moses, M.B. Leon, J.J. Popma, P.J. Fitzgerald, D.R. Holmes, C. O'shaughnessy, R.P. Caputo, D.J. Kereiakes, D.O. Williams, P.S. Teirstein, Sirolimus-eluting stents versus standard stents in patients with stenosis in a native coronary artery, New England Journal of Medicine 349(14) (2003) 1315-1323.

[10] E. Kedhi, K.S. Joesoef, E. McFadden, J. Wassing, C. Van Mieghem, D. Goedhart, P.C. Smits, Second-generation everolimus-eluting and paclitaxel-eluting stents in real-life practice (COMPARE): a randomised trial, The Lancet 375(9710) (2010) 201-209.

[11] M. Joner, A.V. Finn, A. Farb, E.K. Mont, F.D. Kolodgie, E. Ladich, R. Kutys, K. Skorija, H.K. Gold, R. Virmani, Pathology of drug-eluting stents in humans: delayed healing and late thrombotic risk, Journal of the American College of Cardiology 48(1) (2006) 193-202.

[12] H. Tamai, K. Igaki, E. Kyo, K. Kosuga, A. Kawashima, S. Matsui, H. Komori, T. Tsuji, S. Motohara, H. Uehata, Initial and 6-month results of biodegradable poly-1-lactic acid coronary stents in humans, Circulation 102(4) (2000) 399-404.

[13] S. Nishio, K. Kosuga, K. Igaki, M. Okada, E. Kyo, T. Tsuji, E. Takeuchi, Y. Inuzuka, S. Takeda, T. Hata, Long-term ( $>10$ years) clinical outcomes of first-in-human biodegradable poly1-lactic acid coronary stents: Igaki-Tamai stents, Circulation 125(19) (2012) 2343-2353. [14] R. Gao, Y. Yang, Y. Han, Y. Huo, J. Chen, B. Yu, X. Su, L. Li, H.-C. Kuo, S.-W. Ying, Bioresorbable vascular scaffolds versus metallic stents in patients with coronary artery disease: ABSORB China trial, Journal of the American College of Cardiology 66(21) (2015) 2298-2309. [15] M. Peuster, P. Wohlsein, M. Brügmann, M. Ehlerding, K. Seidler, C. Fink, H. Brauer, A. Fischer, G. Hausdorf, A novel approach to temporary stenting: degradable cardiovascular stents produced from corrodible metal—results 6-18 months after implantation into New Zealand white rabbits, Heart 86(5) (2001) 563-569.

[16] M. Peuster, C. Hesse, T. Schloo, C. Fink, P. Beerbaum, C. von Schnakenburg, Long-term biocompatibility of a corrodible peripheral iron stent in the porcine descending aorta, Biomaterials 27(28) (2006) 4955-4962.

[17] Q. Feng, D. Zhang, C. Xin, X. Liu, W. Lin, W. Zhang, S. Chen, K. Sun, Characterization and in vivo evaluation of a bio-corrodible nitrided iron stent, Journal of Materials Science: Materials in Medicine 24(3) (2013) 713-724. 
[18] H. Hermawan, H. Alamdari, D. Mantovani, D. Dube, Iron-manganese: new class of metallic degradable biomaterials prepared by powder metallurgy, Powder Metallurgy 51(1) (2008) 38-45. [19] A. Francis, Y. Yang, S. Virtanen, A. Boccaccini, Iron and iron-based alloys for temporary cardiovascular applications, Journal of Materials Science: Materials in Medicine 26(3) (2015) 138.

[20] M. Moravej, A. Purnama, M. Fiset, J. Couet, D. Mantovani, Electroformed pure iron as a new biomaterial for degradable stents: In vitro degradation and preliminary cell viability studies, Acta Biomaterialia 6(5) (2010) 1843-1851.

[21] M. Moravej, F. Prima, M. Fiset, D. Mantovani, Electroformed iron as new biomaterial for degradable stents: Development process and structure-properties relationship, Acta biomaterialia 6(5) (2010) 1726-1735.

[22] F. Nie, Y. Zheng, S. Wei, C. Hu, G. Yang, In vitro corrosion, cytotoxicity and hemocompatibility of bulk nanocrystalline pure iron, Biomedical Materials 5(6) (2010) 065015. [23] Y. Qi, H. Qi, Y. He, W. Lin, P. Li, L. Qin, Y. Hu, L. Chen, Q. Liu, H. Sun, Strategy of metal-polymer composite stent to accelerate biodegradation of iron-based biomaterials, ACS applied materials \& interfaces 10(1) (2017) 182-192.

[24] J. Cheng, T. Huang, Y. Zheng, Relatively uniform and accelerated degradation of pure iron coated with micro-patterned Au disc arrays, Materials Science and Engineering: C 48 (2015) 679687.

[25] W. Lin, L. Qin, H. Qi, D. Zhang, G. Zhang, R. Gao, H. Qiu, Y. Xia, P. Cao, X. Wang, Longterm in vivo corrosion behavior, biocompatibility and bioresorption mechanism of a bioresorbable nitrided iron scaffold, Acta biomaterialia 54 (2017) 454-468.

[26] B. Heublein, R. Rohde, V. Kaese, M. Niemeyer, W. Hartung, A. Haverich, Biocorrosion of magnesium alloys: a new principle in cardiovascular implant technology?, Heart 89(6) (2003) 651-656.

[27] P. Zartner, R. Cesnjevar, H. Singer, M. Weyand, First successful implantation of a biodegradable metal stent into the left pulmonary artery of a preterm baby, Catheterization and Cardiovascular Interventions 66(4) (2005) 590-594.

[28] D. Schranz, P. Zartner, I. Michel-Behnke, H. Akintürk, Bioabsorbable metal stents for percutaneous treatment of critical recoarctation of the aorta in a newborn, Catheterization and Cardiovascular Interventions 67(5) (2006) 671-673. 
[29] D. Persaud-Sharma, A. McGoron, Biodegradable magnesium alloys: a review of material development and applications, Journal of Biomimetics, Biomaterials and Tissue Engineering, Trans Tech Publ, 2011, pp. 25-39.

[30] F. Witte, N. Hort, C. Vogt, S. Cohen, K.U. Kainer, R. Willumeit, F. Feyerabend, Degradable biomaterials based on magnesium corrosion, Current opinion in solid state and materials science 12(5-6) (2008) 63-72.

[31] Y. Chen, Z. Xu, C. Smith, J. Sankar, Recent advances on the development of magnesium alloys for biodegradable implants, Acta biomaterialia 10(11) (2014) 4561-4573.

[32] X. Gu, Y. Zheng, Y. Cheng, S. Zhong, T. Xi, In vitro corrosion and biocompatibility of binary magnesium alloys, Biomaterials 30(4) (2009) 484-498.

[33] M. Haude, R. Erbel, P. Erne, S. Verheye, H. Degen, D. Böse, P. Vermeersch, I. Wijnbergen, N. Weissman, F. Prati, Safety and performance of the drug-eluting absorbable metal scaffold (DREAMS) in patients with de-novo coronary lesions: 12 month results of the prospective, multicentre, first-in-man BIOSOLVE-I trial, The Lancet 381(9869) (2013) 836-844.

[34] G.B. Lim, DREAMS of a bioabsorbable stent coming true, Nature Reviews Cardiology 10(3) (2013).

[35] R. Waksman, Promise and challenges of bioabsorbable stents, Catheterization and Cardiovascular Interventions 70(3) (2007) 407-414.

[36] C. Indolfi, S. De Rosa, A. Colombo, Bioresorbable vascular scaffolds - basic concepts and clinical outcome, Nature Reviews Cardiology 13(12) (2016) 719.

[37] P.K. Bowen, J. Drelich, J. Goldman, Zinc exhibits ideal physiological corrosion behavior for bioabsorbable stents, Advanced materials 25(18) (2013) 2577-2582.

[38] P.K. Bowen, E.R. Shearier, S. Zhao, R.J. Guillory, F. Zhao, J. Goldman, J.W. Drelich, Biodegradable metals for cardiovascular stents: from clinical concerns to recent $\mathrm{Zn}$-Alloys, Advanced healthcare materials 5(10) (2016) 1121-1140.

[39] P.K. Bowen, R.J. Guillory II, E.R. Shearier, J.-M. Seitz, J. Drelich, M. Bocks, F. Zhao, J. Goldman, Metallic zinc exhibits optimal biocompatibility for bioabsorbable endovascular stents, Materials Science and Engineering: C 56 (2015) 467-472.

[40] D. Pierson, J. Edick, A. Tauscher, E. Pokorney, P. Bowen, J. Gelbaugh, J. Stinson, H. Getty, C.H. Lee, J. Drelich, A simplified in vivo approach for evaluating the bioabsorbable behavior of candidate stent materials, Journal of Biomedical Materials Research Part B: Applied Biomaterials 100(1) (2012) 58-67. 
[41] S. Zhao, C.T. McNamara, P.K. Bowen, N. Verhun, J.P. Braykovich, J. Goldman, J.W.

Drelich, Structural characteristics and in vitro biodegradation of a novel Zn-Li alloy prepared by induction melting and hot rolling, Metallurgical and Materials Transactions A 48(3) (2017) 12041215.

[42] J. Niu, Z. Tang, H. Huang, J. Pei, H. Zhang, G. Yuan, W. Ding, Research on a Zn-Cu alloy as a biodegradable material for potential vascular stents application, Materials Science and Engineering: C 69 (2016) 407-413.

[43] E. Mostaed, M. Sikora-Jasinska, A. Mostaed, S. Loffredo, A. Demir, B. Previtali, D.

Mantovani, R. Beanland, M. Vedani, Novel Zn-based alloys for biodegradable stent applications: design, development and in vitro degradation, journal of the mechanical behavior of biomedical materials 60 (2016) 581-602.

[44] X. Liu, J. Sun, F. Zhou, Y. Yang, R. Chang, K. Qiu, Z. Pu, L. Li, Y. Zheng, Micro-alloying with $\mathrm{Mn}$ in $\mathrm{Zn}-\mathrm{Mg}$ alloy for future biodegradable metals application, Materials \& Design 94 (2016) 95-104.

[45] C. Wang, Z. Yu, Y. Cui, Y. Zhang, S. Yu, G. Qu, H. Gong, Processing of a novel Zn alloy micro-tube for biodegradable vascular stent application, Journal of Materials Science \& Technology 32(9) (2016) 925-929.

[46] E. Mostaed, M. Sikora-Jasinska, J.W. Drelich, M. Vedani, Zinc-based alloys for degradable vascular stent applications, Acta biomaterialia 71 (2018) 1-23.

[47] S. Zhao, J.-M. Seitz, R. Eifler, H.J. Maier, R.J. Guillory II, E.J. Earley, A. Drelich, J. Goldman, J.W. Drelich, Zn-Li alloy after extrusion and drawing: structural, mechanical characterization, and biodegradation in abdominal aorta of rat, Materials Science and Engineering: C 76 (2017) 301-312.

[48] T. Nguyen, A. Cipriano, R.G. Guan, Z.Y. Zhao, H. Liu, In vitro interactions of blood, platelet, and fibroblast with biodegradable magnesium-zinc-strontium alloys, Journal of Biomedical Materials Research Part A 103(9) (2015) 2974-2986.

[49] A. Kafri, S. Ovadia, J. Goldman, J. Drelich, E. Aghion, The suitability of Zn-1.3\% Fe alloy as a biodegradable implant material, Metals 8(3) (2018) 153.

[50] P.K. Bowen, J.M. Seitz, R.J. Guillory, J.P. Braykovich, S. Zhao, J. Goldman, J.W. Drelich, Evaluation of wrought $\mathrm{Zn}-\mathrm{Al}$ alloys $(1,3$, and $5 \mathrm{wt} \% \mathrm{Al})$ through mechanical and in vivo testing for stent applications, Journal of Biomedical Materials Research Part B: Applied Biomaterials 106(1) (2018) 245-258. 
[51] A. Jarzębska, M. Bieda, J. Kawałko, P. Koprowski, K. Sztwiertnia, W. Pachla, M. Kulczyk, A new approach to plastic deformation of biodegradable zinc alloy with magnesium and its effect on microstructure and mechanical properties, Materials Letters 211 (2018) 58-61.

[52] M. Sikora-Jasinska, E. Mostaed, A. Mostaed, R. Beanland, D. Mantovani, M. Vedani, Fabrication, mechanical properties and in vitro degradation behavior of newly developed $\mathrm{ZnAg}$ alloys for degradable implant applications, Materials Science and Engineering: C 77 (2017) 11701181.

[53] L. Mao, G. Yuan, S. Wang, J. Niu, G. Wu, W. Ding, A novel biodegradable Mg-Nd-Zn-Zr alloy with uniform corrosion behavior in artificial plasma, Materials Letters 88 (2012) 1-4.

[54] H. Jin, S. Zhao, R. Guillory, P.K. Bowen, Z. Yin, A. Griebel, J. Schaffer, E.J. Earley, J.

Goldman, J.W. Drelich, Novel high-strength, low-alloys Zn-Mg $(<0.1 \mathrm{wt} \% \mathrm{Mg})$ and their arterial biodegradation, Materials Science and Engineering: C 84 (2018) 67-79.

[55] M. Krystýnová, P. Doležal, S. Fintová, M. Březina, J. Zapletal, J. Wasserbauer, Preparation and Characterization of Zinc Materials Prepared by Powder Metallurgy, Metals 7(10) (2017) 396. 


\section{Understanding zinc material corrosion characteristics and the foreign body response progression in the arterial environment ${ }^{12}$}

\subsection{Introduction}

The concept of constructing fully bioresorbable stents (BRSs) to open occluded arteries using non-toxic degradable materials has inspired extensive research over the past two decades, with an emphasis placed on polymer-based materials $\stackrel{1}{*}$. While multiple polymerbased fully degradable BRS have proceeded through early clinical trials in the US and are beginning to obtain FDA approval ${ }^{2}$, the development of stents manufactured from metallic materials has largely been restricted to pre-clinical studies $\stackrel{1 b}{ }$. This has been the case despite the order-of-magnitude mechanical strength advantage that metals derive over polymers and their similarity to conventional stents in terms of deployment and mechanical characteristics.

Magnesium has been extensively investigated for endovascular stenting and is the only biometallic stent studied in the clinical setting. The benefits of magnesium-based stents include low thrombogenicity, excellent biocompatibility to neighboring cells and tissue, and good target lesion failure rates at 6 months, especially when coated with an antiproliferative drug 3 . However, early generation magnesium stents were found to have high degradation rates and even the $2^{\text {nd }}$ generation stents have late lumen loss rates much higher than standard DESs and some of the polymer-based BRSs $\stackrel{4}{*}$. Though studied less in recent years, iron-based materials have the favorable profile of producing low to moderate inflammatory responses and possess excellent mechanical properties $\underline{5}$, but produce voluminous corrosion products that build up in the artery and repel neighboring cells and biological matrices ${ }^{6}$. The serious limitations of iron and magnesium and the intense focus on surmounting them with incremental material modifications have delayed

\footnotetext{
${ }^{1}$ This chapter contains material previously published in ACS Biomaterials Science and Engineering

${ }^{2}$ References in Section 1 follow the ACS author guidelines.
} 
developmental efforts for bioabsorbable metallic stents relative to polymers. Recently, zinc and its alloys were introduced as stent materials that do not experience the

limitations seen with iron and magnesium ${ }^{7}$. Zinc could potentially break through the considerable developmental hurdles facing absorbable biometals (for a recent review of zinc and its progress towards becoming a candidate material for endovascular stents, refer to $\stackrel{1 b}{)}$.

In contrast to an inflammatory response generated around a biostable material, which is directed primarily against the base material — metals and their stable oxide layer in the case of bare metal stents, or polymers in drug-eluting variants - the inflammatory response to a corrodible metal may also be impacted by the corrosion behavior and the products thereby generated. To date, most studies have focused on the in vitro

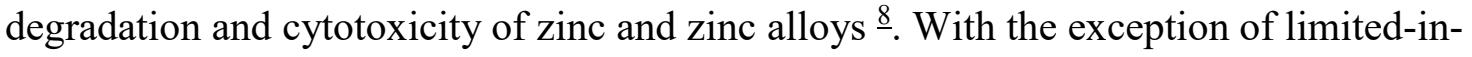
scope in vivo work performed by our group $\underline{1 b}, \underline{7}, \underline{9}$, a detailed investigation into the cellular and tissue interactions with metallic based corrosion products for a degradable metal remains largely unexplored $\stackrel{1 \mathrm{~b}}{ }$. Due to the complex biochemical and cellular constituents present in the physiological environment that impact the generation and progression of corrosion products, as well as their integration and ultimate break down and clearance, a long-term in vivo evaluation is essential to clarify the biological response.

The present work was undertaken to characterize the chronic inflammatory response to zinc by drawing detailed comparisons between different zinc-based materials displaying a range of corrosion behaviors. We describe the cell/tissue remodeling events that take place on the active tissue-material interface of degrading zinc implants in order to understand the inflammatory progression. Through a direct comparison it may be possible to gauge the inflammation response severity and clarify how corrosion behavior may regulate inflammatory pathways. This type of analysis may eventually allow for an improved design of zinc-based stent materials with the goal of minimizing the severity of chronic inflammatory responses. 


\subsection{Experimental}

\subsubsection{Materials and reagents}

$0.25 \mathrm{~mm}$ diameter wire of $99.99+$ wt.\% (4N) zinc was purchased from Goodfellow Corporation. $\mathrm{Zn}-\mathrm{Al}$ alloy ingots were purchased from Metropolitan Alloy Co. (Detroit, MI) at concentrations of $0,1,3$, and $5.5 \mathrm{wt}$. \% Al. The $\mathrm{Zn}$ stock used for fabrication was special high grade (SHG) $\sim 99.7 \%$ zinc per ASTM B6-13. Ingots were gravity cast, in air, into flat steel molds. The pancake ingots received measured approximately $100 \times 70 \times$ $10 \mathrm{~mm}$. Alloying with aluminum substantially improved the mechanical properties of the zinc material (the detailed microstructure and mechanical properties characterization are included in a separate contribution ${ }^{10}$ ). Table 1-1 provides the list of impurities and their concentrations in both $4 \mathrm{~N}$ and SHG Zn.

Table 1-1 - Impurity levels in 4N and SHG Zn

\begin{tabular}{|c|c|c|}
\hline \multirow{2}{*}{ Impurity } & \multicolumn{2}{|c|}{ Concentration (ppm) } \\
\hline & $4 \mathrm{~N}^{\mathrm{a}}$ & $\mathrm{SHG}^{\mathrm{b}}$ \\
\hline $\mathrm{Ag}$ & 5 & $<2$ \\
\hline $\mathrm{Al}$ & $\mathrm{n} / \mathrm{s}$ & $<2$ \\
\hline As & $\mathrm{n} / \mathrm{s}$ & $<2$ \\
\hline $\mathrm{Cd}$ & 7 & $<2$ \\
\hline $\mathrm{Cu}$ & 5 & 7 \\
\hline $\mathrm{Fe}$ & 25 & 371 \\
\hline $\mathrm{Mg}$ & $\mathrm{n} / \mathrm{s}$ & $<2$ \\
\hline $\mathrm{Mn}$ & $<1$ & 3 \\
\hline $\mathrm{Pb}$ & 15 & 16 \\
\hline $\mathrm{Si}$ & $<1$ & $\mathrm{n} / \mathrm{a}$ \\
\hline
\end{tabular}

${ }^{a}$ Per specification; non-specified impurities are labeled " $\mathrm{n} / \mathrm{s}$ "

${ }^{\mathrm{b}}$ Measured via inductively coupled plasma optical emission spectroscopy; non-analyzed impurities are labeled "n/a"

Electro-discharge machining was used to fabricate SHG and Zn-Al strips measuring approximately $12 \mathrm{~mm}$ long $\times 300 \mu \mathrm{m}$ wide $\times 300 \mu \mathrm{m}$ thick from the rolled foil. The 
strips were electropolished using a stirred bath of $120 \mathrm{~mL}$ deionized water, $885 \mathrm{~mL}$ ethyl alcohol (200-proof - Pharmco Aaaper, Brookfield, CT), 100 mL n-butyl alcohol (99.9\% Fisher Scientific, Fair Lawn, NJ), $250 \mathrm{~g}$ anhydrous zinc chloride (reagent grade Avantor, Center Valley, PA), and $109 \mathrm{~g}$ aluminum trichloride hexahydrate $(97+\%$ Sigma Aldrich, St. Louis, MO) at a constant potential of $10 \mathrm{~V}$ for 90 seconds 11 . Due to the electropolishing, the thickness of strips was reduced to $220-270 \mu \mathrm{m}$ and their rectangular cross-sectional dimension aligned closely with that of a wire and are hereafter referred to as wires.

Staining reagents included eosin Y disodium salt, acetic acid (99.7\%), toluidine blue (powdered), phosphotungstic acid solution 10\% w/v, phosphomolybdic acid hydrate solution, biebrich scarlet acid-fuchsin solution, analine blue solution, absolute ethanol, xylene substitute, Gill's No. 3 hematoxylin solution, hematoxylin (powdered), anhydrous iron (III) chloride (powder 99.99\%), Lugol's iodine Solution phosphate buffered saline (PBS), phosphate buffered saline with bovine serum albumin (PBS-BSA), and Eukitt quick-hardening mounting medium; all were obtained from Sigma Aldrich. Hydrochloric acid ( $\mathrm{HCl})(37 \% ; 12.2 \mathrm{M})$ was obtained from EMPROVE (Darmstadt, Germany). Reagent grade acetone was obtained from Pharmco-Aaper.

Primary antibodies included anti-CDd68 (ab125212), anti-Cd11b (ab8879) all purchased from Abcam (Cambridge, Massachussetts). Secondary antibodies include goat anti-rabbit IgG alexa fluor 488 (ab150077), donkey anti-rabbit IgG alexa fluor 555 (ab150074) purchased from Abcam, and anti-CD163 (sc-58965) purchased from Santa Cruz Biotechnology (Dallas, Texas). Streptavidin alexa fluor 633 conjugate (S-21375) was purchased from Life Technologies (Carlsbad, California). Biotinylated goat anti rabbit IgG (ab64256), biotinylated goat anti mouse IgG, and endogenous avidin/biotin blocking kit (ab64212) were all purchased from Abcam. Goat serum (G9023), 4', 6-diamidino-2phenylindole dihydrochloride (DAPI), Triton X-100, phosphate buffered saline (pH 7.4 containing $1 \% \mathrm{BSA} w / \mathrm{v}$ ) were all purchased from Sigma-Aldrich. PermaFluor mountant was purchased from Thermo-Scientific (Waltham, Massachussetts). 


\subsubsection{In vivo implantation and cryo-sectioning}

The zinc implant wires including 4N, SHG, Zn-1Al, Zn-3Al, and Zn-5Al were cleaned in acetone and then sterilized in $70 \%$ (v/v) ethyl alcohol for 20 to $30 \mathrm{~min}$ prior to implantation. A surgical protocol developed recently was employed to implant two wires of the same material composition into the abdominal aorta wall of an adult SpragueDawley rat $\underline{6}$. Briefly, the wires were used to penetrate the adventitial layer of the abdominal aorta, after which the wire was advanced within the arterial wall for the full length of the specimen. We have found that the wall environment is more conducive to biocorrosion activity whereas blood contact from a luminal implantation tends to passivate the metal surface and reduce corrosion. Thus, the wall implantation was employed to increase the bioactivity of the metal surface and elicit the very inflammatory responses that we were interested in investigating. The actual inflammatory response to a stent made from the same material may therefore be less severe than what has been found in the present study. The diameter of the wires is approximately twice that of a stent strut of the same material, thereby introducing a greater challenge to the local arterial environment in terms of material degradation and corrosion product clearance. The SHG, $\mathrm{Zn}-1 \mathrm{Al}$, and $\mathrm{Zn}-3 \mathrm{Al}$ alloy wires were extracted at four time points, including 1.5, 3, 4.5, and 6 Months. The $\mathrm{Zn}-5 \mathrm{Al}$ alloy wires were extracted at 1.5, 3, 3.5, and 6 months. The $4 \mathrm{~N}$ wires were extracted at 13 time points, including 1-10, 12, 15, and 20 months. One of the two wires implanted into each rat was used for metallographic and corrosion rate analysis, while the second wire was used for inflammatory characterization work $(\mathrm{n}=28$ rats). All animals survived the wire implantation surgery and made a full recovery. All animal experiments were approved by the animal care and use committee (IACUC) of Michigan Technological University.

At the indicated time points, wires were excised for histological evaluation and immunofluorescent labeling by collecting the entire segment of host artery that contained the implant. To facilitate cross-sectioning, the lumen of the collected artery was filled with PolyFreeze mounting medium (Sigma Aldrich) by syringe perfusion. Explanted arteries were placed into plastic molds, surrounded with PolyFreeze mounting medium, and snap- 
frozen in liquid nitrogen. Samples were then stored at $-80^{\circ} \mathrm{C}$ until cryo-sectioning. Frozen samples were cross-sectioned at a $10 \mu \mathrm{m}$ thickness with a Thermo-Scientific HM $550 \mathrm{P}$ cryostat. Tissue sections were placed on warm VWR Histobond (Radnor, PA) slides and fixed in $-20^{\circ} \mathrm{C}$ absolute ethanol for $60 \mathrm{~s}$ or in $4 \%$ formaldehyde (Sigma Aldrich) and washed three times in PBS (Sigma Aldrich) for 5 min prior to staining.

\subsubsection{SEM preparation and backscattered imaging}

A JEOL JSM-6400 (Peabody, MA) scanning electron microscope equipped with a dSpec automation system (Geller MicroÅnalytical Laboratory; Topsfield, MA) was used for examining the explant cross sections. Imaging of the carbon-coated specimens was conducted at $10 \mathrm{kV}$ accelerating voltage at a reduced working distance using a backscattered electron detector. The acquired backscattered electron images were analyzed with imageJ (National Institute of Mental Health; Bethesda, Maryland) to yield cross sectional area measurements. The bright zinc portion of the image was selected by thresholding, in which only the area containing the brightest pixels was measured. From these cross sectional area measurements, a metal cross sectional area reduction was calculated for each image. The resulting measurements were averaged to yield an estimated area reduction.

\subsubsection{Hematoxylin and eosin}

Slides with fixed tissue sections were rinsed in deionized water for 5 min to clear any residual PBS. Gills-3 hematoxylin solution was pipetted onto each slide for 2-5 min, or until over-stained (determined by visual examination). Slides were then dipped in a 900 $\mathrm{mL}$ bath of distilled water that had been acidified to $\mathrm{pH}=1.8-2.0$ by adding $\mathrm{HCl}$. After 5 dips in the acidic bath, the samples were rinsed in distilled water for 1-5 min. Slides were then washed twice with 95\% ethanol (v/v). The slides were counterstained with Eosin Y working solution (0.25\%), which was micro-pipetted onto each slide for $45 \mathrm{~s}$. immediately after counterstaining, the slides were rinsed and dehydrated in two changes of absolute ethanol for $5 \mathrm{~min}$ apiece. The slides were then cleared twice with xylene 
substitute for $5 \mathrm{~min}$, and mounted under a cover slip using Eukitt mounting medium. Samples were imaged using an Olympus BX51, DP70 bright-field microscope (Upper Saucon Township, PA).

\subsubsection{Toluidine blue}

A Toluidine Blue stock solution was prepared by dissolving $1 \mathrm{~g}$ of toluidine blue in $70 \%$ ethanol. A working solution was prepared by using $5 \mathrm{~mL}$ of the stock solution and $45 \mathrm{~mL}$ of a $1 \%$ sodium chloride solution with the $\mathrm{pH}$ adjusted to 2.4 using $\mathrm{HCl}$ or $\mathrm{NaOH}$. The slides were then hydrated in three changes of DiH20 for 5 min each and stained in the working solution for $5 \mathrm{~min}$. The slides were then dipped 3 times in $95 \%$ ethanol and 5 times in $100 \%$ ethanol to quickly dehydrate. Slides were then dipped 20 times in each of two clearing solutions - a 1:1 xylene substitute/acetone and a pure xylene substitute mixture - mounted in resinous mounting medium, and imaged with an Olympus BX51, DP70 bright-field microscope.

\subsubsection{Immuno-fluorescence}

Biological tissue surrounding the metal implant became impregnated with an autofluorescent corrosion product. To remove the background fluorescence due to the corrosion product and improve the overall quality of immuno-fluorescent labeling, slides were immersed in a $1 \%(\mathrm{v} / \mathrm{v})$ acetic acid solution for $1 \mathrm{~h}$ to dissolve the corrosion product. Slides were then rinsed in 4 changes of PBS for 5 min each and incubated in a $0.2 \%$ Triton X-100 solution in PBS for 30 min, followed by immersion in PBS-BSA and a $0.2 \%$ Triton X-100 solution (blocking buffer) for $30 \mathrm{~min}$. If biotinylated secondaries were used, avidin/biotin blocking was performed for 15 min each with the endogenous avidin/biotin blocking kit purchased from Abcam, as well as the appropriate IgG. Primaries were then incubated overnight on the slides at $4^{\circ} \mathrm{C}$ in a $1: 100$ dilution, followed by 3 washes in blocking buffer for 5 min each. For CD163 and CD11b antibodies, a concentration of 1:500 was used. Secondaries were incubated on the slides at room temperature in 1:200 concentrations for $1 \mathrm{~h}$. Three washes in blocking buffer were 
performed for 5 min each and the slides were incubated with a DAPI working solution for $1 \mathrm{~min}(1 \mu \mathrm{l} / \mathrm{mL}$ DAPI solution). Slides were then rinsed in 2 changes of PBS and mounted with Perma-Fluor aqueous mounting medium and imaged with appropriate fluorescent filters.

\subsubsection{Immunofluorescent Quantification}

For quantification of immunofluorescent staining, a minimum of 3 tissue sections were taken of each sample separated by at least $100 \mu \mathrm{m}$, and stained with the aforementioned procedures. Sections were then imaged using the appropriate filters at 100X normal magnification, and uploaded to MetaMorph imaging software. The entire inflammatory exudate was included in the area selected for automatic pixel counting. Data was recorded and graphed using Excel 2013. 


\subsection{Results}

\subsubsection{Backscattered imaging and cross sectional area reduction calculations}
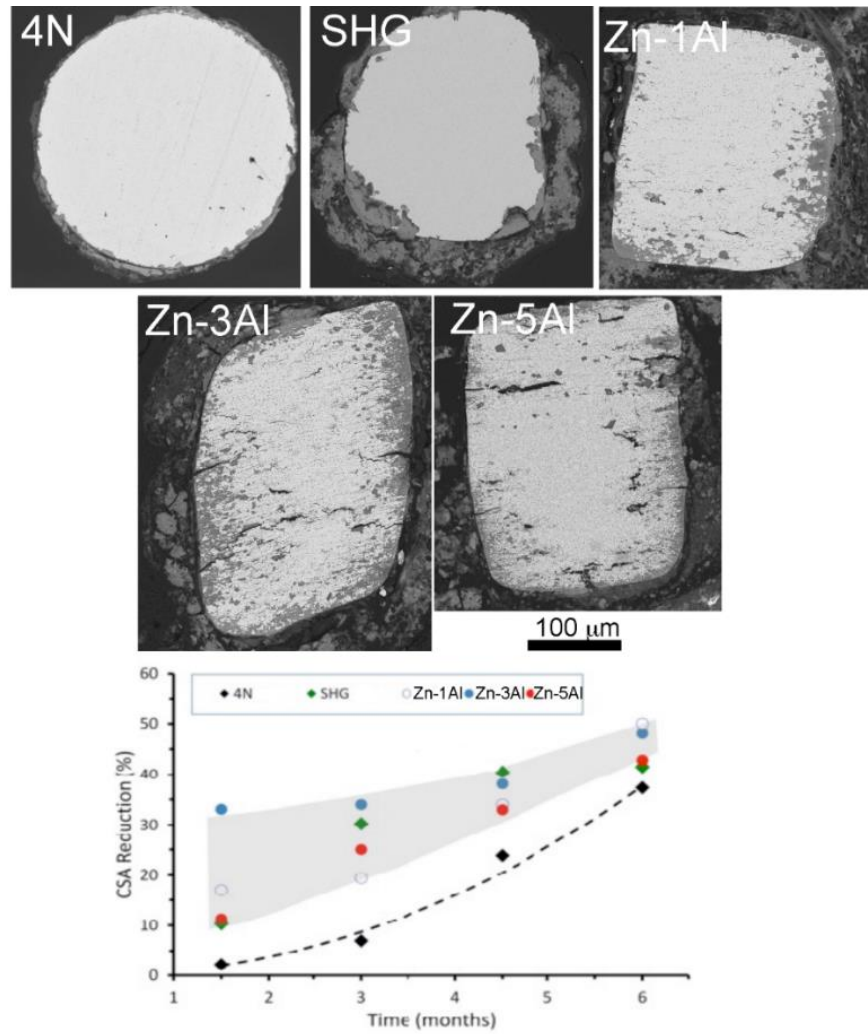

Figure 1-1. Backscattered imaging and cross-sectional area reduction calculation for the five evaluated zinc metal compositions, $4 \mathrm{~N}$ wire and the four strip materials. The backscattered images shown are for the 5 compositions at 1.5 months post-implantation, while the cross sectional area reductions shown in the graph include samples at $1.5,3,4.5$, and 6 months.

The SHG, Zn-1Al, Zn-3Al and Zn-5Al metal wires had the same surface finish after electropolishing, with oxide film thickness between 100 and $200 \mathrm{~nm}$. Only the $4 \mathrm{~N}$ wire had a native oxide film, produced during wire manufacturing, but this film had a similar thickness as presented earlier ${ }^{12}$. Quality of the surface finish influences the early stage of 
biodegradation in the first few weeks after implantation as discussed in the cited reference, but has no effect on corrosion of the $\mathrm{Zn}$ implant in later stages, as discussed in this contribution.

Fig. 1-1 shows examples of metallographic cross sections of 1.5-month specimens. Metallic portion of the explant is represented by the brightest area whereas oxidized metal is in a grey color. These metallographic cross sections revealed sharply varying corrosion behavior between the five zinc compositions that were evaluated. The $4 \mathrm{~N}$ wire experienced a high resistance to corrosion at this time. The SHG experienced a similar mode of corrosion, with a significantly higher cross sectional area reduction of metallic portion relative to the $4 \mathrm{~N}$ material. The $\mathrm{Al}$ alloys experienced a similar cross sectional area reduction as the SHG, but the surface corrosion mode shifted to intergranular attack.

\subsubsection{Hematoxylin and Eosin}
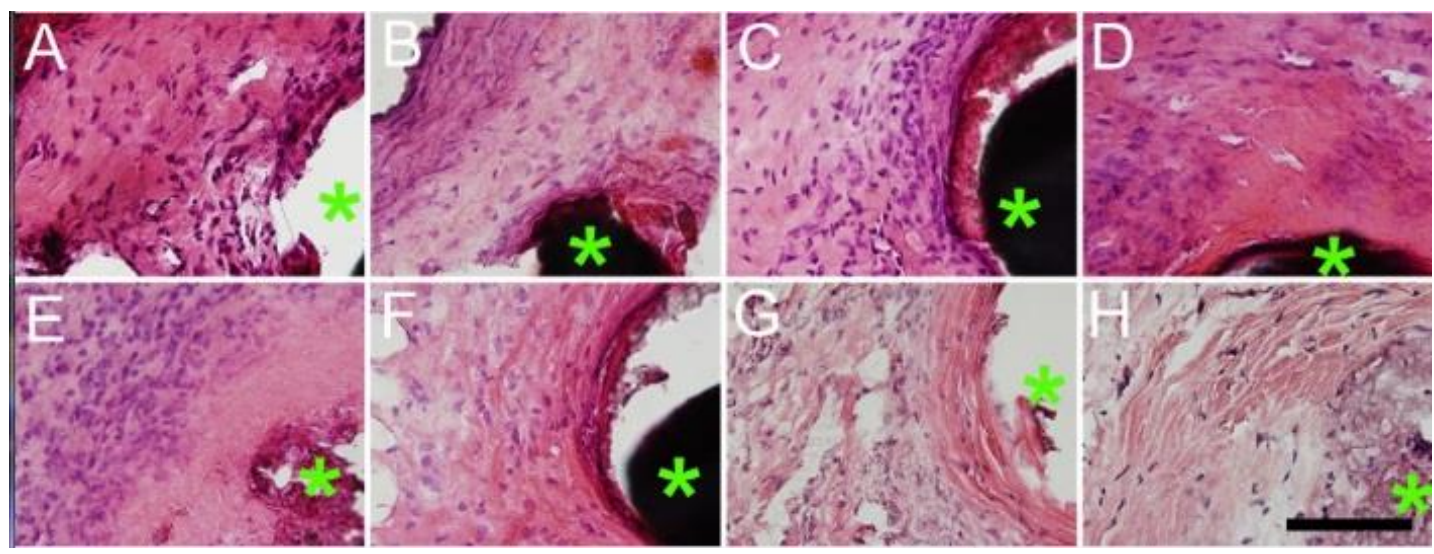

Fig. 1-2. Representative H\&E stains of cross-sections containing the explanted artery with the $4 \mathrm{~N}$ wire implant. Panels $\mathrm{A}-\mathrm{H}$ represent months $1,2,4,5,6,8,9$, 20 , respectively. The green asterisk in each image denotes the wire or corrosion layer location. The scale bar at lower right is $100 \mu \mathrm{m}$. 
The inflammatory response progression against the 4N wire implantations from 1 to 20 months is shown in Fig. 1-2. Panel A shows a small amount of cellular alignment around the metallic implant with mononuclear infiltrates into the surrounding tissue and negligible corrosion of the metallic implant. Panels B and C (2 and 4 months) show progression towards a dense, non-porous corrosion product occupying the space at the original interface between biological tissue and metallic wire, as well as an increase in cellular density at the material interface. Panels D and E (5 and 6 months) show areas near the interface of the implant that have become devoid of nuclei and contain irregular nuclear features. Irregular nuclear features decrease dramatically in the peripheral tissue, with no abnormalities in the media or intima of the artery for each time point. Panels F, $\mathrm{G}$ and $\mathrm{H}(8,9$, and 20 months respectively) depict an increase in fibrotic deposition around the implant, as well as localized chronic inflammation inside the fibrotic capsule and around the interface Increased image size and identification of important cells/tissue remodeling events are shown in Fig.s1-S3 and 1-S4. 


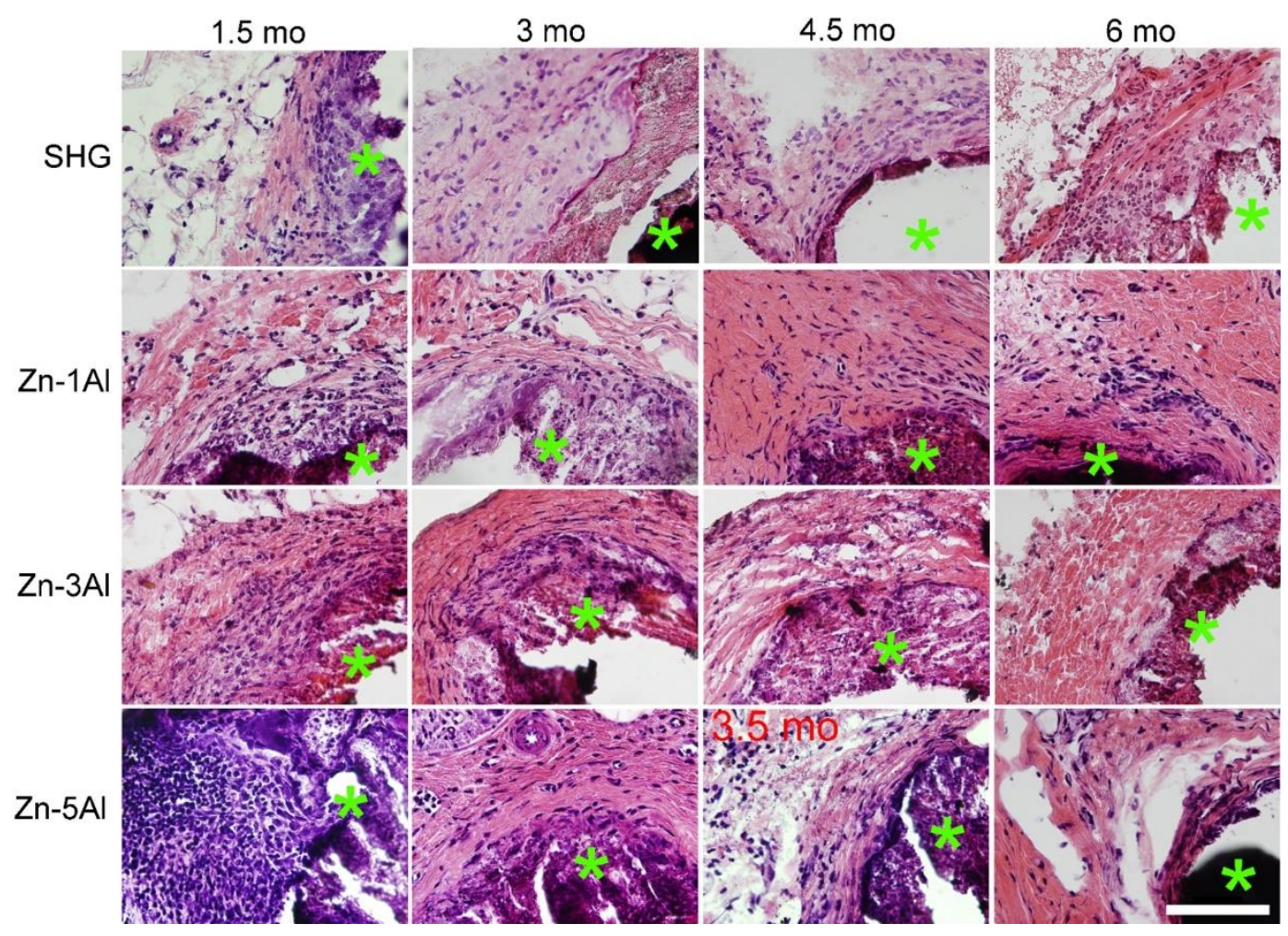

Fig. 1-3. Representative $H \& E$ stains of cross-sections containing the explanted SHG, Zn-1Al, Zn-3Al, and Zn-5Al wire implants within the arterial wall over 1.5 6 months, and $\mathrm{Zn}-5 \mathrm{Al}$ at 1.5, 3, 3.5, 6 months post implantation. The green asterisk denotes the wire or corrosion layer position. The scale bar at lower-right is $100 \mu \mathrm{m}$.

The progression of the inflammatory response against SHG, $\mathrm{Zn}-1 \mathrm{Al}$, and $\mathrm{Zn}-3 \mathrm{Al}$, implants at $1.5,3,4.5$, and 6 months, as well as at $1.5,3$, and 3.5 months for Zn- $8 \mathrm{~A} 1$ is shown in Fig. 1-3. A large corrosion layer is observed for the SHG specimens at 1.5 and 3 months, replete with macrophages and an irregular material - tissue interface. An increase in flocculent corrosion product infiltrated with viable cells was identified on H\&E stains for SHG at 4.5 and 6 months. Tissue deposition is within the original collagenous capsule at 6 months. An increased amount of corrosion relative to $4 \mathrm{~N}$ and a more intense cellular infiltration relative to SHG is observed within the corrosion layer for $\mathrm{Zn}-1 \mathrm{Al}$ at 1.5 months. At 3 months, an increase in capsule density is seen for $\mathrm{Zn}-1 \mathrm{Al}$ 
in Fig. 1-3, as well as infiltrating cells with fragmented nuclei. For $\mathrm{Zn}-1 \mathrm{Al}$ at 4.5 months, an increase in fibroblasts and cellular organization is evident, with active chronic inflammatory cells present inside the corrosion layer. A highly organized collagenous capsule is apparent at 6 months for $\mathrm{Zn}-1 \mathrm{Al}$, with neovascularization and localized chronic inflammation. For the Zn-3Al series of implants, a high degree of corrosion and cellular infiltration with an ill-defined tissue material interface at 1.5 months is shown in Fig. 1-3. At 3 months, a highly organized and cellularized collagenous capsule formed around $\mathrm{Zn}$ $3 \mathrm{Al}$, with fibroblasts present at the interface and inflammatory cells inside the interface and corrosion layer. Some nuclear fragmentation is also visible in the cells present within the corrosion layer. At 4.5 months for $\mathrm{Zn}-3 \mathrm{Al}$, an increase in nuclear disparity was noticed inside the corrosion layer. Collagen bundles of fibrotic character surround the implant, with an increasing amount of acellularity relative to the 3 month specimen. At 6 months for $\mathrm{Zn}-3 \mathrm{Al}$, a dense collagenous capsule with sparse amounts of cells surrounds the implant. Macrophages still impregnate the corrosion layer and an active tissuematerial interface is still evident. The Zn-5Al 1.5 month specimen displayed the most intense initial inflammatory reaction of all the specimens, with a high cell density at and around the material tissue interface. At 3 months, concentric cellular alignment was formed around the corrosion layer interface, which increased in its regularity at 3.5 months. A dense, fibrous capsule was built around the corrosion layer for the 3.5 month time point. For more detailed descriptions, see Fig.s 1-S5-1-S8. 

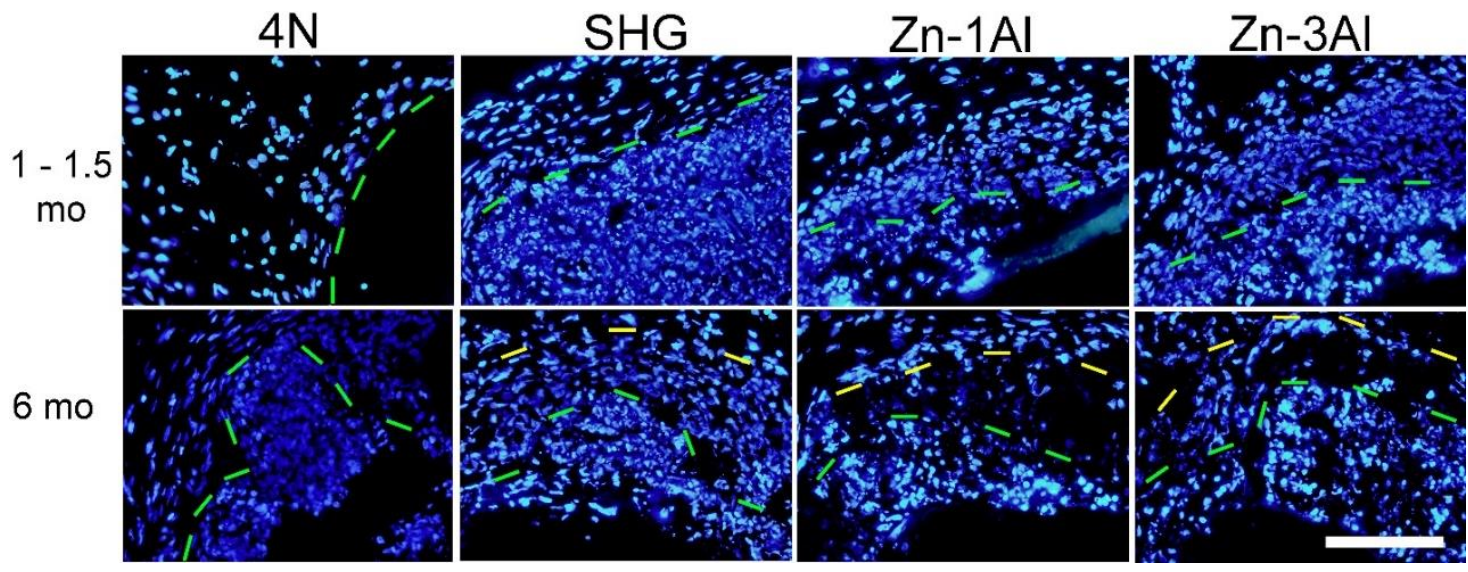

\subsubsection{DAPI Staining}

Fig. 1-4. Representative DAPI images showing corrosion layer and tissue interface for $4 \mathrm{~N}, \mathrm{SHG}, \mathrm{Zn}-1 \mathrm{Al}$, and $\mathrm{Zn}-3 \mathrm{Al}$ implants at $1-1.5$ and 6 months. The green dashed line identifies the interface between corrosion layer and tissue. The yellow dashed line identifies the location of the old interface. The scale bar at lower-right is $100 \mu \mathrm{m}$.

Because it was sometimes challenging to clearly visualize cells present within and near the corrosion layer in the H\&E stains, DAPI staining was used to help improve the nuclear characterization of this cell population for the different zinc materials. Representative images for each zinc composition, excluding $\mathrm{Zn}-5 \mathrm{Al}$, are shown at approximately 1-1.5 months and 6 months (Fig. 1-4). The $4 \mathrm{~N}$ material exhibited a slight presence of intact mononuclear cells directly at the rigid interface at 1 month. At 6 months, a dense and uniform population of both intact and dying cells was present within the corrosion layer. Dying cells are evident throughout this layer by the appearance of nuclear fading, shrinkage, and fragmentation. Cells in the surrounding tissue are highly aligned. For SHG zinc at 1.5 months, a dense and uniform population of both intact and dying cells was present within the corrosion layer. Surrounding cells displayed some degree of alignment as well as the general absence of inflammatory cells. At 6 months, 
the corrosion layer-tissue interface appeared to move closer to the zinc implant, potentially indicative of a dynamic interface. The space between the new and old interface was uniformly populated with viable cells, indicative of healthy tissue regeneration having taken place. At 1.5 months for $\mathrm{Zn}-1 \mathrm{Al}$ and $\mathrm{Zn}-3 \mathrm{Al}$, a high density of mononuclear cells is present immediately adjacent to the tissue interface with the corrosion layer, symptomatic of an intense inflammatory response in the surrounding tissue. The coverage of cells within the corrosion layer was not uniform, with open spaces evident at numerous locations, revealing an environment that may be toxic to cells. These cells displayed a greater degree of intense nuclear staining relative to what was seen surrounding the SHG material, consistent with nucleic acid condensation $\frac{13}{}$. At 6 months, the interface had advanced closer to the implant. However, the space between the old and new interface was largely devoid of cell nuclei, symptomatic of poor tissue regeneration. In general, the results indicate moderately healthy tissue regeneration around the SHG implant in contrast to toxicity in combination with poor tissue regeneration around the $\mathrm{Zn}-1 \mathrm{Al}$ and $\mathrm{Zn}-3 \mathrm{Al}$ implants due to apparent differences in the inflammatory response. Greater detail is presented in Fig.s 1-S9 and 1-S10. 


\subsubsection{Toluidine Blue}
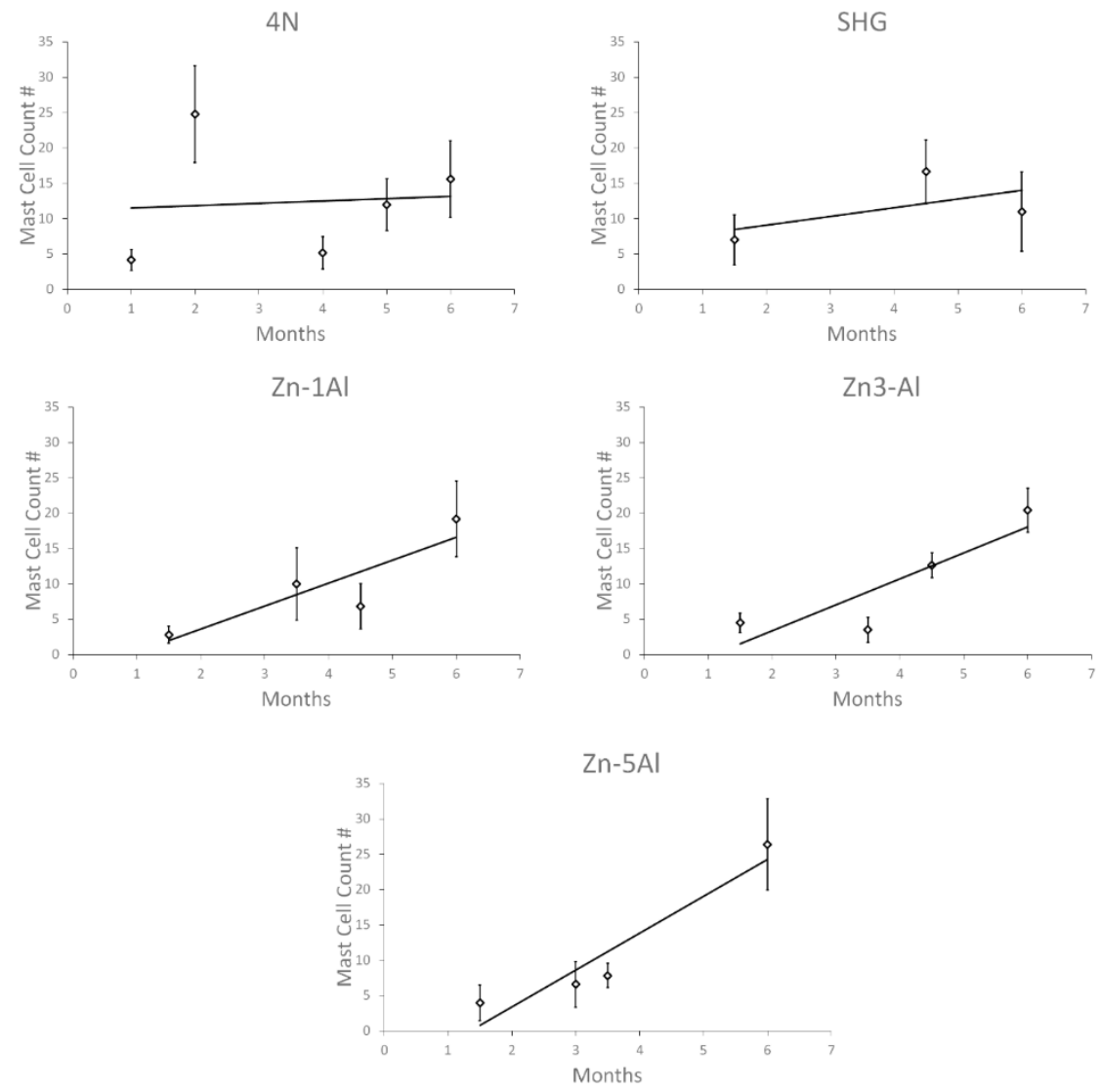

Fig. 1-5. Quantification of average number of mast cells via multiple sections per sample.

Mast cell counting via toluidine blue staining was performed for all alloy compositions up to 6 months as shown in Fig. 1-5. A slightly higher mast cell average is seen for the initial months of the SHG specimen, relative to the $\mathrm{Al}$ alloys, with a slightly increasing trend over a period of 1 to 6 months. The average number of mast cells counted for the $\mathrm{Zn}-\mathrm{Al}$ alloys increased more sharply over 6 months compared with the $4 \mathrm{~N}$ and SHG material, revealing a temporal increase of inflammatory intensity for the $\mathrm{Zn}-\mathrm{Al}$ alloys. Average mast cell counts for the SHG specimen at 6 months $( \pm 11 \pm 6)$ is slightly lower when compared to $4 \mathrm{~N}$ at the same time $( \pm 16 \pm 5)$. The $\mathrm{Zn}$-Al alloys' average mast cell 
count after 6 months was $\pm 19 \pm 5, \pm 20 \pm 3$, and $\pm 6.526 \pm 7$ for $2 \mathrm{Al}, 4 \mathrm{Al}$, and $8 \mathrm{Al}$ respectively. The $\mathrm{Zn}-\mathrm{Al}$ alloy counts were all higher than for the SHG and $4 \mathrm{~N}$ samples.

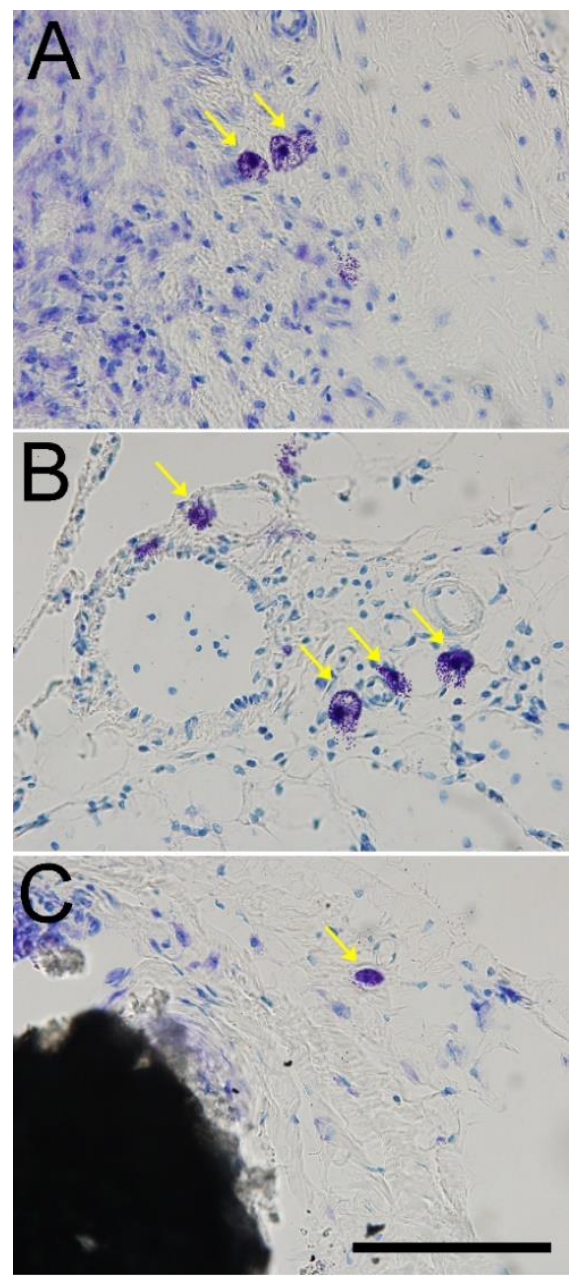

Fig. 1-6. Toluidine Blue staining of $4 \mathrm{~N}$ (panels $\mathrm{A}, \mathrm{B}$ ), and SHG (panel C) implants at 6 months. The image shown in panel $A$ is from a region in close proximity to the implant. The image shown in panel $B$ is from the peripheral tissue, approximately $1 \mathrm{~mm}$ from the implant surface. Yellow arrows denote mast cells. The scale bar in the lower image is $100 \mu \mathrm{m}$.

Toluidine Blue staining revealed the presence of active (degranulating) mast cells near the 6 month $4 \mathrm{~N}$ implant (Fig. 6, panel A) and in the surrounding tissue (panel B). Panel C in Fig. 1-6 shows a positive but inactive (quiescent) mast cell staining near the 6-month 
SHG implant. All mast cells (degranulating and quiescent) were included in counting for completeness.

\subsubsection{CD68,CD11b, and CD163 fluorescence}

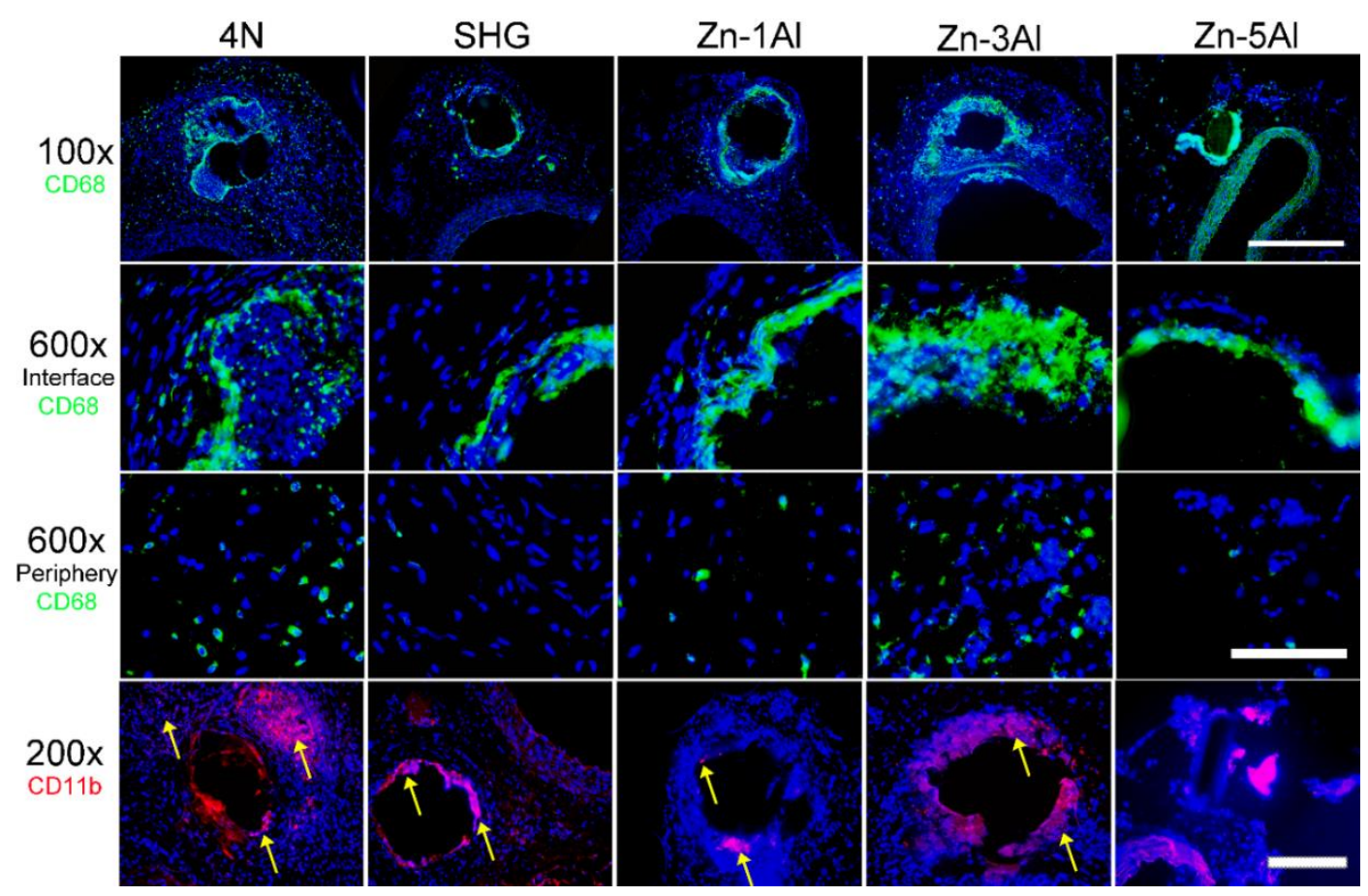

Fig. 1-7. CD68 (green) and CD11/b (red) labeling of cross-sections containing the explanted artery and the $4 \mathrm{~N}, \mathrm{SHG}, \mathrm{Zn}-1 \mathrm{Al}, \mathrm{Zn}-3 \mathrm{Al}$ and $\mathrm{Zn}-5 \mathrm{Al}$ compositions at 6 months. Cell nuclei were counterstained with DAPI (blue). High magnification CD68 images at the tissue-material interface and in the peripheral tissue are shown in the 2nd and 3rd rows for each specimen. The scale bar for the 100x

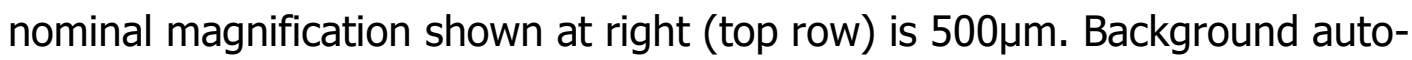
fluorescence of the elastin rich media layer is seen in the $\mathrm{Zn}-5 \mathrm{Al}$ specimen. The scale bar for the $600 \times$ nominal magnification images shown at right ( 3 rd row) is $100 \mu \mathrm{m}$. The scale bar for the $200 x$ nominal magnification images shown at the right of the bottom row is $200 \mu \mathrm{m}$. 
Fluorescent antibody labeling was performed to identify the response of $\mathrm{CD} 68^{+}$and $\mathrm{CD}_{11 \mathrm{~b}^{+}}$cells to the different zinc compositions (Fig. 1-7). The CD68 glycoprotein is highly expressed in monocyte/macrophage populations $(\mathrm{M} \phi)^{14} \mathrm{~A}$ large population of CD68 ${ }^{+}$cells surrounded both the $4 \mathrm{~N}$ and $\mathrm{Zn}-3 \mathrm{Al}$ implants. A thick $\mathrm{CD} 68^{+}$cell layer was established at the interface of these two materials, although the positive cell layer at the $\mathrm{Zn}-3 \mathrm{Al}$ interface was more continuous and uniform relative to a more irregular $4 \mathrm{~N}$ interface that did not surround the entire metal specimen. The media layer in the $\mathrm{Zn}-3 \mathrm{Al}$ specimen showed intense positive staining for CD68. A more detailed examination revealed substantial damage to the ECM of the media layer in the $\mathrm{Zn}-3 \mathrm{Al}$ section (data not shown). In contrast, the SHG and Zn-1 Al compositions exhibited moderate CD68 labeling at the interface and sharply decreasing numbers of positive cells in peripheral tissues, although a modest increase in positive labeling was apparent in the $\mathrm{Zn}-1 \mathrm{Al}$ periphery relative to SHG. 

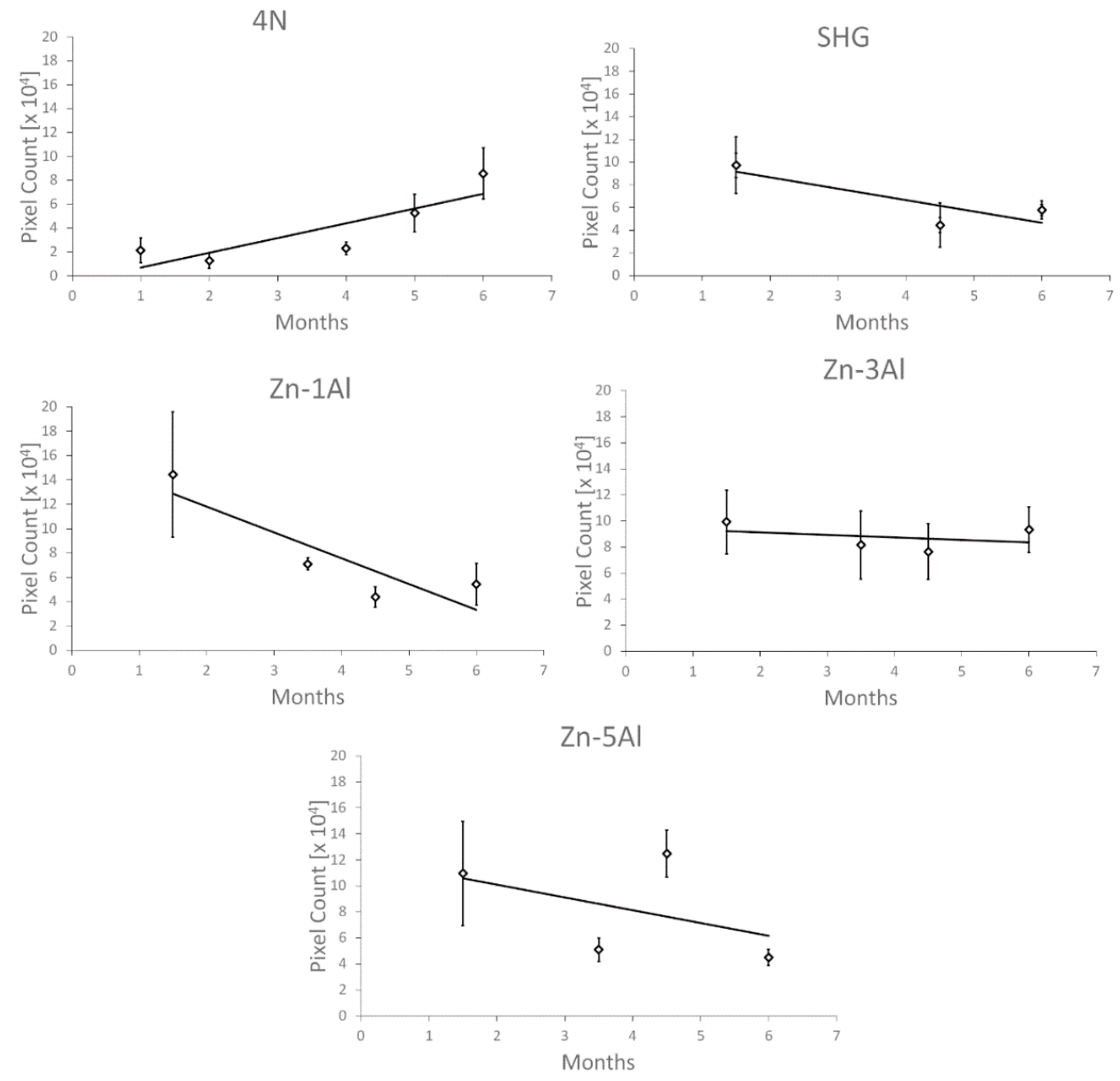

Fig. 1-8. Quantification of CD68 staining using average pixel counting of multiple tissue sections per sample.

Trends of relative CD68 expression for each sample was evaluated and quantified (Fig. 18). Interestingly, the $4 \mathrm{~N} \mathrm{CD} 68$ expression at the 1 month time point, $(2 \pm 1) \times 10^{4}, \times$ $10^{4} \pm \times 10^{4}$ is substantially lower than the SHG response at 1.5 months, $(10+3) \times 10^{4}, \times$ $10^{4} \pm \times 10^{4}$ )with increasing vs. decreasing counts respectively.

CD11b and CD163 labeling was performed on all specimens and quantified up to 6 months (Fig.s S1 and S2). Cells that express CD11b are mostly granulocytes, such as neutrophils. Macrophages can also express CD11b, but to a reduced extent relative to 
neutrophils. The presence and distribution of $\mathrm{CD} 11 \mathrm{~b}^{+}$cells provide fundamental information related to the stage of inflammation at a wound site. In our 6 month samples, $\mathrm{CD} 11 \mathrm{~b}^{+}$cells were present near the interface of the $4 \mathrm{~N}$ and $\mathrm{Zn}-3 \mathrm{Al}$ compositions, while the SHG and $\mathrm{Zn}-1 \mathrm{Al}$ compositions experienced a reduced presence of $\mathrm{CD} 11 \mathrm{~b}$ positive cells both near and far from the interface. $\mathrm{CD} 163^{+}$cells were believed to be alternatively activated macrophages, commonly known as M2 macrophages, and generally decrease the inflammatory response around a wound site. They also encourage tissue repair by releasing urea, polyamines, and ornithine ${ }^{15}$. Tissue with a more resolved state of inflammation around an implant will contain $\mathrm{CD} 163^{+}$cells, while a chronic inflammatory response will most likely have little to no $\mathrm{CD} 163^{+}$cells $\frac{16}{}$. In our 6 month samples, $\mathrm{CD} 163^{+}$cells were seen very far away from the $4 \mathrm{~N}$ implant, but not near the interface. The SHG and $\mathrm{Zn}-1 \mathrm{Al}$ implants were the only implants to show $\mathrm{CD}_{163^{+}}$cells at the tissue-implant interface. The $\mathrm{Zn}-3 \mathrm{Al}$ implant was largely void of $\mathrm{CD} 163^{+}$cells.

At 6 months, the 4N and SHG materials elicited a similar expression level (positive pixel count, Fig. 1-S1) of CD163 labeling, $(4 \pm 2) \times 10^{4}$ and $(3 \pm 2) \times 10^{4}$, respectively. $\times 10^{4} \pm$ $\times 10^{4} \times 10^{4} \pm 2.4 \times 10^{4}$ This was the case, although they demonstrated dissimilar early time point expression values, $(0.5 \pm 0.3) \times 10^{4}$ and $(4 \pm 2) \times 10^{4}$, respectively. $\times$ $10^{3} \pm \times 10^{3} \times 10^{4} \pm \times 10^{4} \mathrm{The} \mathrm{Zn}-1 \mathrm{Al}, \mathrm{Zn}-3 \mathrm{Al}$, and $\mathrm{Zn}-5 \mathrm{Al}$ alloys elicited relatively low expression levels of CD163 at 6 months, $(1.6 \pm 0.9) \times 10^{4},(1.2 \pm 0.3) \times 10^{4}$, and $(2.4 \pm$ $0.5) \times 10^{4}$ respectively. $\times 10^{4} \pm \times 10^{3} \times 10^{4} \pm \times 10^{3} \times 10^{4} \pm \times 10^{3}$

According to Fig. 1-S2, the $\mathrm{Zn}-1 \mathrm{Al}, \mathrm{Zn}-3 \mathrm{Al}$, and $\mathrm{Zn}-5 \mathrm{Al}$ alloys elicited an initially high CD11b presence, $(5 \pm 1) \times 10^{4},(4 \pm 1) \times 10^{4},(5 \pm 1) \times 10^{4}, \times 10^{4} \pm \times 10^{4} \times 10^{4} \pm \times 10^{4} \times$ $10^{4} \pm \times 10^{4}$ when compared to the $4 \mathrm{~N}$ and SHG materials, $(3 \pm 1) \times 10^{4}$ and $(1.8 \pm$ $0.5) \times 10^{4}$, respectively. $\times 10^{4} \pm \times 10^{4} \times 10^{4} \pm 4.7 \times 10^{3}$ 


\subsection{Discussion}

An interesting finding in this work is that the inflammatory response evolution differed dramatically between five zinc-dominant materials with minor additions of non-zinc impurities or alloying elements. The compositional impurities that ranged from $\sim 0.01$ (4N) to $\sim 0.3 \%$ (SHG) produced dramatic differences in inflammation. This was the case despite a similar cross sectional area reduction by 6 months for all examined materials. Surprisingly, the highest purity $4 \mathrm{~N}$ zinc implant elicited the strongest negative immune response. Minor impurity introductions of $0.3 \mathrm{wt} \%$ (SHG) produced the best performing zinc implant of the five, with a highly biocompatible immune response. The results demonstrate that the immune response against zinc implants was not related to zinc per $s e$, but is rather shaped by the material's intrinsic corrosion behavior. Because zinc $4 \mathrm{~N}$ corrosion rate at early times was substantially lower than that of SHG zinc, we speculate that the initial corrosion rate may be a critical regulator of inflammation.

An analysis of the histological and metallographic data provides insight into the potential mechanism by which the corrosion rate may regulate the inflammatory response. For the 4N composition, a necrotic response at 5, 6, and 8 months (Fig. 1-2, panels D-F respectively) is highly indicative of inflammation-induced matrix remodeling around the implant. The necrosis is believed to have occurred due to the generation of a mature fibrotic capsule between 4 and 6 months, coupled with what appear to be intense, localized corrosion events that damage the fibrotic capsule. We have reported that the stable $\mathrm{ZnO}$ layer present around the $4 \mathrm{~N}$ implant increases into a bulk corrosion product at 4.5 and 6 months ${ }^{9}$. The temporal trend of necrosis appearing by 5 months and a bulk $\mathrm{ZnO}$ product appearing by 4.5 months is associated with our recent report that the $4 \mathrm{~N}$

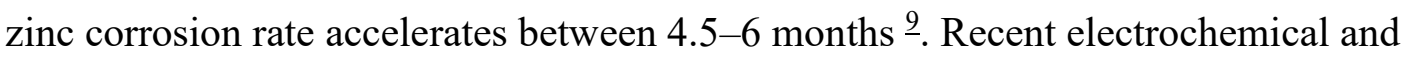
thermodynamic characterization of zinc corrosion as a function of $\mathrm{pH} \frac{17}{}$ has identified ionic zinc as the most stable species of zinc at low $\mathrm{pH}$ conditions $(\mathrm{pH} \sim 1-4) \mathrm{Zn}^{2+17}$, while $\mathrm{ZnO}$ is the most thermodynamically stable phase of zinc at physiological $\mathrm{pH}$. The presence of necrotic tissue at the $4 \mathrm{~N}$ interface (Fig. 1-2) at the time points where we 
found an increased corrosion rate [8]. Such an environment could have materialized if frustrated phagocytosis by foreign body giant cells (FBGCs) and resident $\mathrm{M} \phi \mathrm{s}$ at the $4 \mathrm{~N}$ implant lowered the interfacial $\mathrm{pH}$ to acidic conditions. The resulting ionic $\mathrm{Zn}^{2+}$ release could induce massive cell death near the implant surface, due to a low reported cell

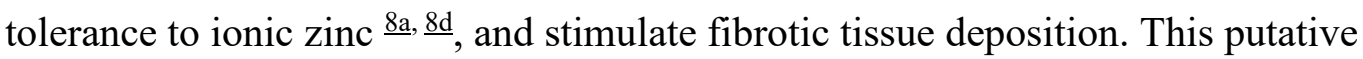
mechanism may explain the widespread cell death seen at 5,6 and 8 months and the ensuing fibrotic capsule growth near the surface of the $4 \mathrm{~N}$ material, at which time points we have also found a high CD68 and CD11b signal. Similar reasoning could also explain the lack of cell death in the SHG material. In this case, downward temporal trending of $\mathrm{CD} 68+\mathrm{M} \phi$ (Fig. 1-8) around the SHG implant may reflect a near neutral $\mathrm{pH}$ interfacial environment and effective $\mathrm{M} \phi$ clearance of porous $\mathrm{ZnO}$ and $\mathrm{ZnOH}$ corrosion products.

It is well known that foreign particle size and scaffold porosity affect $\mathrm{M} \phi$ signaling, phagocytosis, and fusion $\frac{18}{2}$. We speculate that the initial low corrosion rate of the $4 \mathrm{~N}$ material may have elicited macrophage fusion (observed, but not shown) by presenting a rigid interface and eventually large corrosion particles or a low porosity scaffold to the surrounding tissue. $\mathrm{M} \phi$ fusion / foreign body giant cells can cause tissue damage by releasing powerful enzymes and reactive oxygen species in an effort to degrade the material into smaller, digestible particles $\frac{19}{}$. Interestingly, the $4 \mathrm{~N}$ corroding implant elicited the degranulation of mast cells (Fig.s 1-5 and 1-6), The findings of inflammatory events at 6 months for the $4 \mathrm{~N}$ material that are normally associated with acute inflammation (Fig. 1-5) are consistent with the concept of an increased $M \phi$ activity that accelerates corrosion and increases toxicity to cells near the implant surface.

In contrast, the SHG and Zn-Al metals all appear to degrade earlier than the $4 \mathrm{~N}$ material and produce a relatively thick and porous corrosion layer (Fig. 1-3). This also correlates well with temporal CD68 expression, as presented in Fig. 1-8. The porosity is apparent due to the increased consistency of cellular infiltration within the corrosion layer for SHG and the $\mathrm{Zn}-\mathrm{Al}$ alloys. The ability of inflammatory cells to penetrate the corrosion layer at early times may explain the reduced thickness of the fibrous capsule in SHG relative to $4 \mathrm{~N}$. Interestingly, the $4 \mathrm{~N}$ material was the only implant to elicit an acellular fibrotic 
encapsulation response, despite the similar material compositions and preponderance of zinc amongst the SHG and Zn-Al wire implants.

Surprisingly, the SHG material was the only material to elicit a relatively moderate amount of CD163 expression through the entire 6-month implantation time (Fig. 1-S1). The $\mathrm{Al}$ alloys all had consistently low expression of CD163 over the time course, with a large increase of expression over 6 months for the $4 \mathrm{~N}$ material. The consistent presence of CD163+ cells around the SHG material could explain its positive tissue remodeling and integration, while the $4 \mathrm{~N}$ response could be explained as an effort to reconFig. the arterial damage due to the necrotic response shown in Fig. 1-2.

Although cells had penetrated the corrosion layer at early time points in the SHG and ZnAl materials, a decrease in cell viability can be seen from SHG, to Zn-1Al, and then Zn$3 \mathrm{Al}$, respectively, with the $\mathrm{SHG}$ corrosion layer exhibiting the fewest irregular cells. The graded differences in cell viability within the corrosion layer of the $\mathrm{Zn}$ - $\mathrm{Al}$ alloys may explain the increasing capsule thickness from SHG and $\mathrm{Zn}-1 \mathrm{Al}$ to $\mathrm{Zn}-3 \mathrm{Al}$, as well as the lack of a dense collagenous capsule for the SHG material, as seen in Fig. 1-3. Fig. 1-8 clearly shows high initial CD68 presence for the $\mathrm{Al}$ additions, which could be indicative of a highly aggressive macrophage attack in the early weeks of implantation. The graded difference in cell viability was associated with the degree of CD68 labeling, suggesting that $\mathrm{M} \phi$ activity may have contributed to the reduced cell viability within the corrosion layer. Although the presence of aluminum appeared to have elicited a greater inflammatory response, the $\mathrm{Al}^{3+}$ is not believed to be harmful, because there was no indication of toxicity to cells away from the regions of tissue where the macrophages had clustered. Potentially, the aluminum addition may have produced corrosion products (possibly $\mathrm{Al}_{2} \mathrm{O}_{3}$ ) that are too small or chemically stable for efficient phagocytosis, resulting in an increased residence time and build-up of $M \phi s$ in and around the corrosion layer. Another explanation is that the intergranular mode of corrosion may elicit a more robust inflammatory response relative to the surface corrosion experienced by the SHG material. For instance, oxygen gradients established by its transport into the metal interior through grain boundaries may stimulate secretion of harmful reactive oxygen 
species by M $\phi$ s. Lastly, $\mathrm{Zn}-\mathrm{Al}$ corrosion may increase the $\mathrm{pH}$ near the implant $\stackrel{20}{ }$, resulting in cell toxicity due to hydroxide ion transport from the material to the biological tissue.

\subsection{Conclusions}

Zinc implants produced dramatic differences in inflammation in rat arteries. The differences in biocompatibility of zinc materials are attributed in this study to their compositional dissimilarities that cause variations in implant corrosion activities and zinc ion fluxes. We conclude that the corrosion behavior (surface vs. intergranular), corrosion rate, corrosion product porosity, and the presence of a dynamic tissue-material interface regulate the inflammatory progression and remodeling of surrounding and penetrating tissue. $M \phi s$ and their monocyte precursor are known to orchestrate the fibrotic response in an up/down regulatory manner $\frac{21}{2}$, making them the key cells that mediate inflammation and implant degradation. The ability of $M \phi s$ to penetrate and remain viable within the corrosion layer at early times may be of fundamental importance for eliciting long-term biocompatible inflammatory responses around corrodible metals. Future work will aim to further clarify the mechanisms by which zinc metallic implants regulate $M \phi$ activity and viability with the aim to control inflammatory responses around vascular implants made from zinc base materials and predict long-term biocompatibility from early stage corrosion behavior.

\subsection{Acknowledgements}

This work was funded by the National Institutes of Health R15HL129199 and R21EB019118.

\subsection{References}

1. (a) Onuma, Y.; Serruys, P. W., Bioresorbable scaffold the advent of a new era in percutaneous coronary and peripheral revascularization? Circulation 2011, 123 (7), 779-797; (b) Patrick K. Bowen, E. R. S., Shan Zhao, Roger J. Guillory II, Feng Zhao, Jeremy Goldman, and 
Jaroslaw W. Drelich, Cardiovascular stents made of biodegradable zinc-current status. Advanced Healthcare Materials 2016.

2. Rizik, D. G.; Shah, M. G.; Burke, R. F., First US experience following FDA approval of the ABBOTT vascular bioresorbable vascular scaffold for the treatment of coronary artery disease. Catheterization and Cardiovascular Interventions 2016.

3. Heublein, B.; Rohde, R.; Kaese, V.; Niemeyer, M.; Hartung, W.; Haverich, A., Biocorrosion of magnesium alloys: a new principle in cardiovascular implant technology? Heart 2003, $89(6), 651-656$.

4. $\quad$ Erbel, R.; Di Mario, C.; Bartunek, J.; Bonnier, J.; de Bruyne, B.; Eberli, F. R.; Erne, P.; Haude, M.; Heublein, B.; Horrigan, M., Temporary scaffolding of coronary arteries with bioabsorbable magnesium stents: a prospective, non-randomised multicentre trial. The Lancet 2007, 369 (9576), 1869-1875.

5. Moravej, M.; Prima, F.; Fiset, M.; Mantovani, D., Electroformed iron as new biomaterial for degradable stents: Development process and structure-properties relationship. Acta biomaterialia 2010, 6(5), 1726-1735.

6. Pierson, D.; Edick, J.; Tauscher, A.; Pokorney, E.; Bowen, P.; Gelbaugh, J.; Stinson, J.; Getty, H.; Lee, C. H.; Drelich, J., A simplified in vivo approach for evaluating the bioabsorbable behavior of candidate stent materials. Journal of Biomedical Materials Research Part B: Applied Biomaterials 2012, 100 (1), 58-67.

7. Bowen, P. K.; Guillory, R. J.; Shearier, E. R.; Seitz, J.-M.; Drelich, J.; Bocks, M.; Zhao, F.; Goldman, J., Metallic zinc exhibits optimal biocompatibility for bioabsorbable endovascular stents. Materials Science and Engineering: C 2015, 56, 467-472.

8. (a) Emily R. Shearier, P. K. B., Weilue He, Adam Drelich, Jaroslaw Drelich, Jeremy Goldman, and Feng Zhao, In Vitro Cytotoxicity, Adhesion, and Proliferation of Human Vascular Cells Exposed to Zinc ACS Biomaterials Science \& Engineering 2016; (b) Hiebl, B.; Nennig, E.; Schiestel, S.; Kovacs, A.; Jung, F.; Fischer, H., Biocompatibility of a novel zinc stent with a closed-cell-design. Clinical hemorheology and microcirculation 2015, 61 (2), 205-211; (c) Liu, X.; Sun, J.; Yang, Y.; Pu, Z.; Zheng, Y., In vitro investigation of ultra-pure Zn and its mini-tube as potential bioabsorbable stent material. Materials Letters 2015, 161, 53-56; (d) Ma, J.; Zhao, N.; Zhu, D., Endothelial cellular responses to biodegradable metal zinc. ACS Biomaterials Science \& Engineering 2015, 1 (11), 1174-1182; (e) Törne, K.; Larsson, M.; Norlin, A.; 
Weissenrieder, J., Degradation of zinc in saline solutions, plasma, and whole blood. Journal of Biomedical Materials Research Part B: Applied Biomaterials 2015.

9. Bowen, P. K.; Drelich, J.; Goldman, J., Zinc exhibits ideal physiological corrosion behavior for bioabsorbable stents. Advanced Materials 2013, 25 (18), 2577-2582.

10. P.K. Bowen, J.-M. S., R.J. Guillory II, J.P. Braykovich, S. Zhao, J. Goldman, and J.W. Drelich, Evaluation of Wrought $\mathrm{Zn}-\mathrm{Al}$ alloys (1, 3, and $5 \mathrm{wt} \% \mathrm{Al})$ Through Mechanical and In Vivo Corrosion Testing for Stent Applications. Journal of Biomedical Materials Research: Part B - Applied Biomaterials-submitted 2016.

11. Brandes, E.; Smithells, C., Metals reference handbook. Butterworths, London 1983.

12. Drelich*, A. J. D. P. K. B. L. L. J. G. J. W., Importance of Oxide Film in Endovascular Biodegradable Zinc Stents. Surface Innovations 2016.

13. Kapuscinski, J., DAPI: a DNA-specific fluorescent probe. Biotechnic \& Histochemistry 2009.

14. Pulford, K. A.; Sipos, A.; Cordell, J. L.; Stross, W. P.; Mason, D. Y., Distribution of the CD68 macrophage/myeloid associated antigen. International immunology 1990, 2 (10), 973-980.

15. Galván-Peña, S.; O’Neill, L. A., Metabolic reprograming in macrophage polarization. Frontiers in immunology 2014, 5.

16. (a) Brown, B. N.; Valentin, J. E.; Stewart-Akers, A. M.; McCabe, G. P.; Badylak, S. F., Macrophage phenotype and remodeling outcomes in response to biologic scaffolds with and without a cellular component. Biomaterials 2009, 30 (8), 1482-1491; (b) Badylak, S. F.; Valentin, J. E.; Ravindra, A. K.; McCabe, G. P.; Stewart-Akers, A. M., Macrophage phenotype as a determinant of biologic scaffold remodeling. Tissue Engineering Part A 2008, 14 (11), 1835 1842; (c) Moestrup, S. K.; Møller, H. J., CD163: a regulated hemoglobin scavenger receptor with a role in the anti-inflammatory response. Annals of medicine 2004, 36 (5), 347-354.

17. Thomas, S.; Birbilis, N.; Venkatraman, M.; Cole, I., Corrosion of zinc as a function of pH. Corrosion, The Journal of Science and Engineering 2012, 68 (1), 015009-1-015009-9.

18. (a) Champion, J. A.; Walker, A.; Mitragotri, S., Role of particle size in phagocytosis of polymeric microspheres. Pharmaceutical research 2008, 25 (8), 1815-1821; (b) Chono, S.; Tanino, T.; Seki, T.; Morimoto, K., Uptake characteristics of liposomes by rat alveolar macrophages: influence of particle size and surface mannose modification. Journal of pharmacy and pharmacology 2007, 59 (1), 75-80; (c) Foged, C.; Brodin, B.; Frokjaer, S.; Sundblad, A., Particle size and surface charge affect particle uptake by human dendritic cells in an in vitro 
model. International journal of pharmaceutics 2005, 298 (2), 315-322; (d) Shanbhag, A. S.; Jacobs, J. J.; Black, J.; Galante, J. O.; Glant, T. T., Macrophage/particle interactions: effect of size, composition and surface area. Journal of biomedical materials research 1994, 28 (1), 81-90; (e) Xia, Z.; Triffitt, J. T., A review on macrophage responses to biomaterials. Biomedical materials 2006, 1 (1), R1.

19. Anderson, J. M.; Rodriguez, A.; Chang, D. T. In Foreign body reaction to biomaterials, Seminars in immunology, Elsevier: 2008; pp 86-100.

20. Devillers, L.; Niessen, P., The mechanism of intergranular corrosion of dilute zincaluminium alloys in hot water. Corrosion Science 1976, 16 (4), 243IN12251IN13252IN14-250.

21. Wynn, T. A.; Barron, L. In Macrophages: master regulators of inflammation and fibrosis, Seminars in liver disease, NIH Public Access: 2010; p 245. 


\section{Development of novel in vivo methodology to screen degradable materials for biodegradable stent development 34}

\subsection{Introduction}

Zinc alloy development and materials characterization for vascular stent application has been facilitated by a plethora of standardized and inexpensive methods. These methods have been employed over the past several years to generate a number of candidate zinc based alloys for vascular stents [1-3]. In contrast, the biocompatibility evaluation of zinc based degradable biomaterials for vascular applications has been challenged by a lack of standardized procedures at early developmental stages. While materials that have been selected for stent prototyping are commonly evaluated in a large animal stent implantation model using procedures designed for human subjects, such an approach is prohibitive for evaluating early stage materials due to its high cost and requirements of medical-grade facilities and medical expertise. With the steady accumulation of new zinc based materials [2], a standard and inexpensive approach for the biocompatibility testing of degradable metals for vascular applications is urgently needed.

Historically, the degradable metals community has relied upon in vitro evaluation of candidate materials to assess biocompatibility. In this fashion, cell culture approaches dominate the preliminary biological testing, with investigators focusing their attention on either cytotoxic or mechanistic investigations. Cultured cells are exposed to $\mathrm{Zn}^{2+}$ ions [46] or corrosion extracts collected and concentrated from candidate materials that were corroded in one of a number of different corrosion solutions [7-10]. These have become standard approaches that can provide fundamental insight into the interactions between corrosion products and various cell types.

\footnotetext{
${ }^{3}$ This chapter contains material previously published in the Journal of Metals

${ }^{4}$ References in this section follow the author guidelines for JOM
} 
While directly investigating cell responses to corrosion products provides a fundamental understanding of the biologic response, results are not directly applicable to the compatibility of the material in the application environment. An artery is a complex tissue with three main resident cell types (media - vascular smooth muscle cells, intima endothelial cells, and adventitia - fibroblasts). While the cytotoxicity and sub-cellular response of any of these three cell types is important for determining the overall compatibility of the tissue to degrading zinc materials, molecular cross talk, differences in proximity between the three arterial compartments to the implant, and interaction of blood borne cells play an important role in macro-level vascular responses, which cannot be replicated in vitro [11]. For instance, activation of an organism's innate immune system by biomaterial implantation and tissue injury will impact the overall reaction against the material, critically affecting device performance [12]. Furthermore, the corrosion of zinc based materials varies widely between corrosion solutions and physiological conditions. A distinction must be drawn between cell toxicity (obtainable from direct exposure of cultured cells to $\mathrm{Zn}^{2+}$ or corrosion products) and application specific biocompatibility (requiring in vivo assessment of the material in the appropriate physiological environment).

Stents implanted into the arterial environment are expected to restore and maintain continued function of the artery. Harmful events, including smooth muscle cell neointimal proliferation, thrombogenesis, negative arterial remolding, and destructive inflammation contribute to the narrowing of the lumen and restenosis of the stented segment. While measurements of cytotoxicity can contribute to predicting negative stent outcomes, it cannot replace direct application-specific testing. For instance, magnesium and iron biometals perform superbly when measured for cytotoxicity, but struggle in the application environment due to drawbacks in their corrosion rates that eventually lead to device failure $[13,14]$.

In 2012, our group introduced a metal wire implantation model as a cost effective and simplified approach for the biocompatibility evaluation of degradable materials in the vascular environment [15]. Any material that can be drawn into a 0.20-0.25 mm nominal 
diameter wire can be evaluated in this model to investigate material biocompatibility. Implanting a wire into the lumen of the abdominal rat aorta rather than a stent into a pig is promising from a device testing standpoint: the wire affords a simpler geometry without balloon injury, and is less expensive in terms of animal costs, materials preparation, and corrosion analysis.

In this contribution, we aim to describe a cost effective histomorphometric standard for a pipeline-based evaluation of candidate degradable materials using the murine based wire implant model. Herein, wires made from two zinc alloys and pure zinc were implanted into the abdominal aorta of Sprague Dawley rats and collected at 6 and 11 months. Neointima tissue surrounding the wire was measured for area, thickness, and protrusion into the arterial lumen, critical metrics for quantifying vascular application based biocompatibility.

\subsection{Experimental}

\subsubsection{Materials}

Staining reagents include powdered iron hematoxylin (H3136), sodium sulfate pentahydrate $\left(\mathrm{Na}_{2} \mathrm{SO}_{4} \cdot 5 \mathrm{H}_{2} \mathrm{O}\right)$ (753599) anhydrous iron (III) chloride $\left(\mathrm{FeCl}_{3}\right)$ powder 99.99\% (157740), phosphate buffered saline pH 7.4 (PBS, P4417), Lugol's iodine solution(624-71), xylene substitute (78475), Van Gieson solution (HT254), and Eukitt quick hardening mounting medium (03989); all were obtained from Sigma Aldrich(Burlington, MA). 200 proof Ethyl alcohol (absolute ethanol) was obtained from Pharmco-Aaper. Neutral buffered formalin 10\% ( HT501128) was also obtained from Sigma Aldrich.

\subsubsection{Surgical Implantation}

For the present study, a complete histological characterization of metallic wires implanted into the rat abdominal aorta was explored. Pure zinc ( $\mathrm{Zn}$ ) (number of samples) $=10$, zinc-4 wt. \% lithium $(\mathrm{Zn}-4 \mathrm{Li})$ (number of samples) $=10$, and micro-alloyed zinc- 
magnesium (number of samples) $=12$ (either $\mathrm{Zn}-0.01$ wt. \% $\mathrm{Mg}$ or $\mathrm{Zn}-0.1$ wt. \% Mg, hereafter referred to as $\mathrm{Zn}-\mathrm{XMg}$ ) wires were implanted and collected at 6 and 11 months. Two sample sites were evaluated per wire (near both puncture sites for each wire) resulting in $\mathrm{n}=64$. Greater details of the $\mathrm{Zn}, \mathrm{Zn}-4 \mathrm{Li}$, and $\mathrm{Zn}-\mathrm{XMg}$ wires used in the present study can be found in references $[16,17]$.
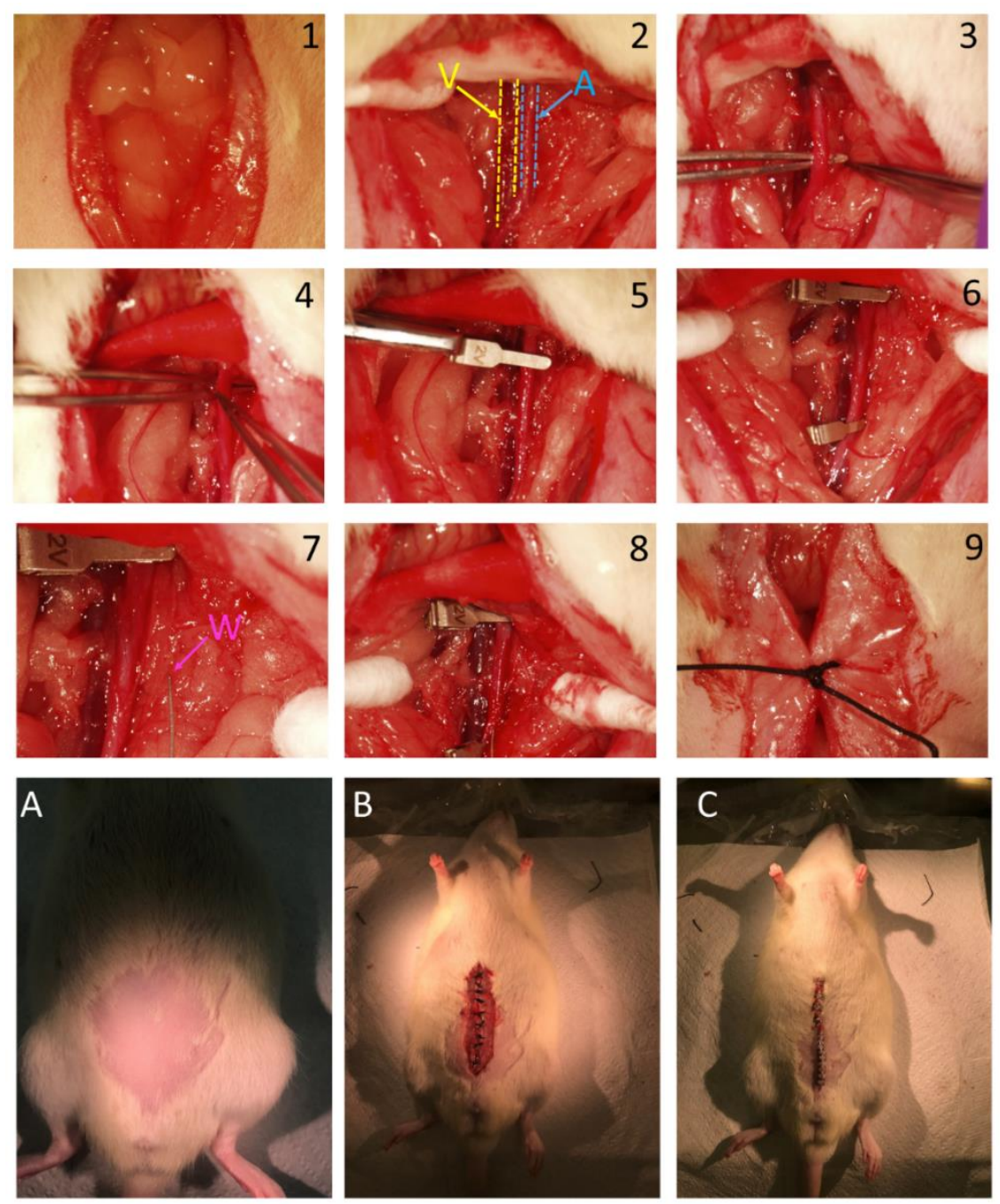

Fig.2-1 Panels 1-9 show the surgical depiction of wire implantation. Yellow "V" and blue " $A$ " denotes the inferior vena cava and descending abdominal aorta respectively. Purple "W" highlights a wire for insertion. Panel A shows the Sprague Dawley rat in the supine position with the abdominal area shaved and 
cleaned before procedure. B shows the abdominal muscle with multiple sutures. C shows the skin clamped with stainless steel staples.

All animal experiments were approved by the Michigan Technological University IUCAC board. Rats were anesthetized in the supine position using an $\mathrm{O}_{2}$ rich isoflurane vaporized gas mixture. The abdomen was shaved and cleaned using antiseptic wipes (Fig.2-1a). For the initial incision, the skin was cut with dissecting scissors through the midline starting approximately $3 \mathrm{~cm}$ above the pelvic region and terminating after approximately $5-8 \mathrm{~cm}$. The abdominal muscle was cut in a similar fashion (step 1 of Fig.1). With the viscera exposed, intestines were carefully pushed aside in order to visualize the caudal descending abdominal aorta and vena cava (step 2). Connective tissue surrounding the vessel was carefully pushed aside with Q-tips to completely expose the vessel. In order to continue with the implantation, blood flow must be briefly interrupted, which is accomplished by placing vascular clamps inferior to the renal arteries and superior to the aortic bifurcation. First, the aorta and vena cava must be carefully separated by using blunted forceps. This is accomplished by carefully poking through and then enlarging an opening between the vessels to fit the clamp. After separation, the proximal clamp is placed first, then the distal clamp applied (steps 3-6). A wire sharpened by angular cuts (using very sharp scissors) is poked into the artery at a location superior to the distal clamp (steps $7 \& 8$ ). After insertion, the wire is advanced within the artery and then punctured out of the artery, producing a second puncture site. The wire is left in place with $\sim 2-3 \mathrm{~mm}$ of wire exteriorized from the artery at both ends. Zinc wires do not need to be secured in place, because corrosion activity embeds the wire within the tissue. If biostable wires are implanted (as controls, for example), the wires must be secured in place to prevent shifting. The vascular clamps are removed (the distal clamp is removed first). The abdominal muscle is sutured together, and the skin clamped (panels b \& c). The rat is allowed to recover under observation, following a subcutaneous injection of butorphanol for pain management. Proper mobility of the lower limbs is used as an indicator of continued abdominal aorta function. All animals survived the procedure. 


\subsubsection{Implant removal and gross examination}

Rats are anesthetized using a vaporized isoflurane and $\mathrm{O}_{2}$ rich mixture. After the subject is deeply anesthetized, the abdominal aorta is isolated from the surrounding tissue. The diaphragm is punctured and the heart is removed from the thoracic cavity to ensure death. The aorta containing the wire is then collected and examined grossly for any obvious abnormalities. The aorta is then placed in a small cryo-mold, surrounded by optimum cryo-temperature cutting medium, snap frozen in liquid nitrogen, and stored at $-80^{\circ} \mathrm{C}$ until cryo-sectioning.

\subsubsection{Tissue preparation and histological evaluation}

Specimens that are snap-frozen can be stored at $-80^{\circ} \mathrm{C}$ for up to one year while maintaining tissue integrity. To prepare for histological evaluation, the implant containing aorta is oriented to ensure a cross-sectional profile of the wire/artery. The artery is then freeze-mounted on a specimen chuck, and placed onto the head of a cryomicrotome for cryo-sectioning. In our experience, a blade angle of 12 degrees and a chamber temperature of $-24^{\circ} \mathrm{C}$ to $-26^{\circ} \mathrm{C}$ is optimal for these samples. A section thickness of 5-10 $\mu \mathrm{m}$ can be reliably produced, but for the present study all sections were taken at a section thickness $\approx 8 \mu \mathrm{m}$. We initially section rapidly through the exteriorized portion of the wire while visualizing the wire in the sample (with a hand-held magnifying lens) as it moves progressively closer to the artery. Once the wire in the specimen can be seen within the wall of the artery, the sample is more carefully sectioned until the wire appears to pass the internal elastic lamina. The wire position in the sample can be verified by inspecting cross sections on an inverted microscope equipped with polarized light filters. When the wire has crossed the internal elastic lamina, sections are collected on slides for later staining, with multiple sections per sample collected. 

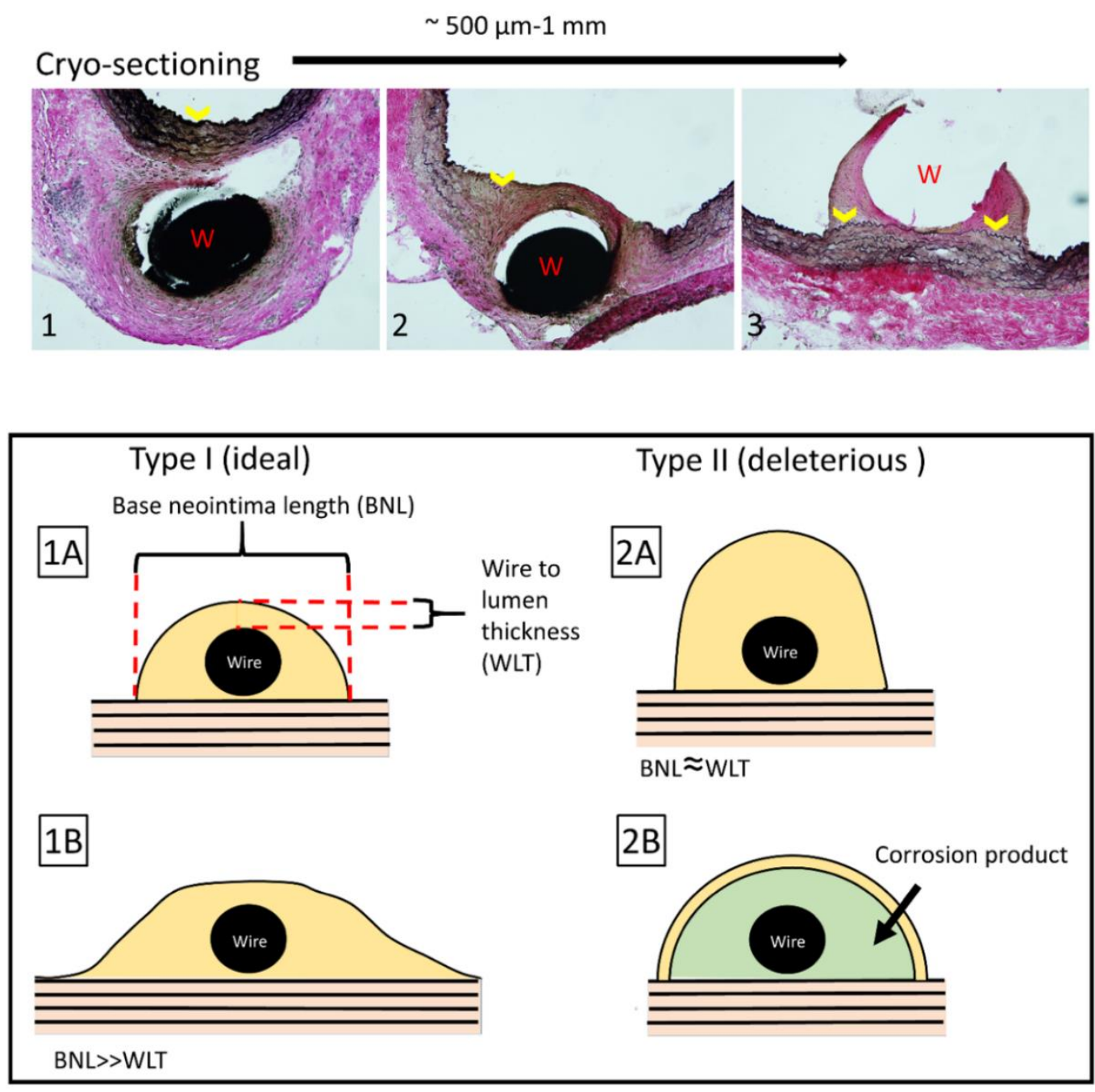

Fig.2-2 VVG staining, Panels 1-3 highlight the sectioning progression of the wire position in relation to the artery. Red " $\mathrm{w}$ " shows the $\mathrm{Zn}$ wire and yellow arrows are positioned at the first elastic fiber. Cartoon depiction displays four most common neointima types, with the explained morphometric measurement locations in relation to the neointimal formation.

This process is seen in Fig.2-2, with clear progression of the wire into the luminal space of the artery. To ensure a representative characterization is performed, cross sections are collected over a distance of $100-500 \mu \mathrm{m}$ into the specimen. As the sample is 
progressively cross-sectioned, the wire may eventually appear to "free float" in the arterial lumen. Cross sections where the wire is not in contact with the artery are not used for histological analysis. Cross sections near the second puncture site can be collected either by flipping the specimen over on the chuck and repeating the procedure described above, or by continuing to section through the sample, although this is more labor intensive. By this method, cross sections near both puncture sites from the implant can be obtained.

\subsubsection{Histological Staining and Morphometric Analysis}

Slides with frozen sections were fixed for $10 \mathrm{~min}$ at room temperature using a bufferedformalin solution. After fixation, the slides were washed 3 times for $5 \mathrm{~min}$ each in phosphate buffered saline. A hematoxylin and eosin stain (H\&E) was carried out as a general stain in order to visualize tissue constituents and qualitatively assess vessel morphology (see the following reference for procedure details [18]). A Veorohoff Van Geison elastic tissue stain (VVG) was carried out to identify the elastic laminae within the artery. This provides anatomical landmarks for the histomorphometric analysis.

For Veorohoff Van Geison staining, slides with tissue sections were fixed in 10\% neutral buffered formalin for 10 minutes. Slides were then washed in three changes of PBS for 5 min each and overstained in Verhoeff's elastic stain for $10 \mathrm{~min}(30 \mathrm{~mL}$ of absolute ethanol and hematoxylin solution $5 \% \mathrm{~m} / \mathrm{v}, 12 \mathrm{~mL}$ of deionized water and $\mathrm{FeCl}_{3}$ solution $10 \% \mathrm{~m} / \mathrm{v}$, and $12 \mathrm{~mL}$ of Lugol's solution). The slides were then differentiated using an inverted optical microscope with a $\mathrm{FeCl}_{3}$ solution diluted to $2 \% \mathrm{~m} / \mathrm{v}$ until elastic fibers in the media layer of the arterial cross- section was well defined, and non-elastic tissue became unstained. The slides were then rinsed in deionized water for $5 \mathrm{~min}$. The slides were placed in a deionized water and $\mathrm{Na}_{2} \mathrm{SO}_{4} \cdot 5 \mathrm{H}_{2} \mathrm{O}$ solution, $5 \% \mathrm{~m} / \mathrm{v}$ for 1 minute and then rinsed in running tap water for $5 \mathrm{~min}$ in order to "blue" the hematoxylin. The slides were counter stained in Van Gieson solution for $3 \mathrm{~m}$ and microscopically differentiated in a $95 \% \mathrm{v} / \mathrm{v}$ ethanol solution until there was a clear distinction between the pink Van Gieson stained adventitia and the brown stained media. The slides were dehydrated in 
two changes of absolute ethanol for five min each. The slides were then optically cleared with two changes of xylene substitute for 5 min each and mounted under a coverslip using Eukitt quick hardening mounting medium. The samples were imaged using an Olympus BX51, DP70 bright-field microscope (Upper Saucon Township, PA).

Neointimal area (NA), wire-to-lumen thickness (WLT), and base neointimal length (BNL) were measured using METAMORPH software. Cross-sections stained with VVG and imaged at 100X normal magnification were used for the measurements. The neointima was considered as tissue surrounding the implant on the luminal side of the first elastic fiber, which was highlighted by VVG staining, with the wire cross sectional area excluded. All neo-tissue growth contacting the implant was included in the measurement, as well as all significant intimal activation in nonadjacent regions. WLT was measured near the apex of the neointima, at the point where the tissue protruded furthest into the lumen. For BNL, the first elastic fiber was traced (below the wire implant) until negligible neointimal activation was observed (Fig.2-2). Multiple sections of each sample were evaluated to ensure a representative depiction of each sample (minimum of 5 sections). Only sections that satisfied quality concerns (section not torn considerably, neointima still intact, low amount of section folding) were measured.

\subsubsection{Zn Accumulation in Organs}

To provide insight into whether zinc is cleared or retained with the body during implant degradation, the organs that play a major role in the distribution, filtering and regulation of the fluid compartment and constituents were analyzed for zinc concentration. Wires were implanted within the abdominal aorta for 6 months, whereupon the heart, blood plasma, liver and kidney were collected. Using a fluorometric zinc quantification assay (ab176725) and a Bradford assay (ab102535), all purchased form Abcam (Cambridge, MA), zinc levels were determined relative to control tissue (collected from non-implant rats), and reported in $\mu \mathrm{g} / \mathrm{mL}$. 


\subsubsection{Statistical Analysis}

Histomorphometric data was uploaded into MATLAB R2018a. A Wilcoxon ranksum test was performed on all test groups within each respective time point (6 or 11 month implantation) relative to the reference $\mathrm{Zn}$ implant. A $\mathrm{p}$ value $<0.05$ was considered significant. Data plotted in boxplot graphs show the upper and lower quartiles (25\%$75 \%$ ) within the boxed region, $0 \%$ and $100 \%$ quantiles within the whiskers, with red '+' as computed outliers. For tissue derived $\mathrm{Zn}^{2+}$ organ concentration, a two-tailed student's t-test with assumed equal variances was used to determine significance between control and test groups. Bar graphs denote sample mean, with error bars in standard deviation. A $p$ value $<0.05$ was considered significant.

\subsection{Results and discussion}

The development of zinc-based metals is advancing in academic institutions although it has not yet reached the clinical stage. The potential for targeting multiple vascular applications as degradable vascular implant materials has sparked intense interest amongst metallurgists and corrosion scientists with expertise in materials development. To the authors' knowledge, most of the in vivo work published to evaluate zinc based materials in the vascular environment has emerged from our group, with a single contribution based on pure Zn stents deployed in the rabbit abdominal aorta by Yang et al, [19]. In order to develop a coherent framework for the early in vivo testing of zinc implant materials, we have provided extensive details of our small animal model, including surgical, tissue sectioning, and histological staining procedures. We have also developed metrics to quantitatively characterize the long-term histological progression of the neointima that forms around zinc based wire implants. The approach used here could become a standard in the field, which would allow direct comparison between biodegradable materials developed by different laboratories throughout the world. The metrics we have developed for the wire model have been adapted from metrics that are used to characterize the neointima that forms around vascular stents. Therefore, these 
metrics may help predict performance of the same material when deployed as a stent in a large animal model.

\subsubsection{General histological presentation}

The evaluation of neointimal characteristics provides critical insight into the biological performance of intraluminal implants. Stent performance is routinely evaluated by specific morphometric characteristics including lumen area, neointimal thickness, neointima area, and internal elastic lamina area [20-22]. The wire implantation model simulates a single stent strut placed within the lumen of an artery, without concomitant radial luminal injury or mechanical excitation of the implant. As it is known that radial force exerted during balloon catheter deployment provokes inflammation and smooth muscle cell proliferation[23], the present model allows us to investigate the host response to the experimental material without confounding effects related to deployment. Classical stent metrics such as lumen area and external elastic lamina area (used to determine positive and negative remodeling) are not useful when using the wire model, due to the wire not engaging the entire artery circumferentially. The characteristics of the developed neointima surrounding the wire implant provides the most useful information relevant to implant performance. Fig.2-2 (lower box) summarizes the various neointima types that dominate most observations in our wire model. Type 1A neointima formations are considered ideal, with a low profile and a stable, mature tissue response to the implanted wire. Type 1B formations are common at longer time points (11 months), and in our experience are believed to be the natural temporal progression of Type 1A formations. Type II formations reflect the negative responses seen in this model. The characteristics of Type $2 \mathrm{~A}$ are assumed to be analogous to negative arterial remodeling of a stented artery, which contributes to luminal area reduction and reduced vascularization. We have seen this response in our previous investigation of $\mathrm{Fe}$ wires and in a more dramatic fashion with PLLA coated $\mathrm{Zn}$ wires $[15,24]$. The failure mode of this formation consists of vascular smooth muscle cell (VSMC) neointima hyperplasia, which we confirmed by alpha actin positive staining (data not published). Type 2B presents as a thin neointima with voluminous corrosion product and a cell rich middle layer. The consistent thin tissue 
surrounding the implant could result in rupture, which is not ideal for stenting applications.

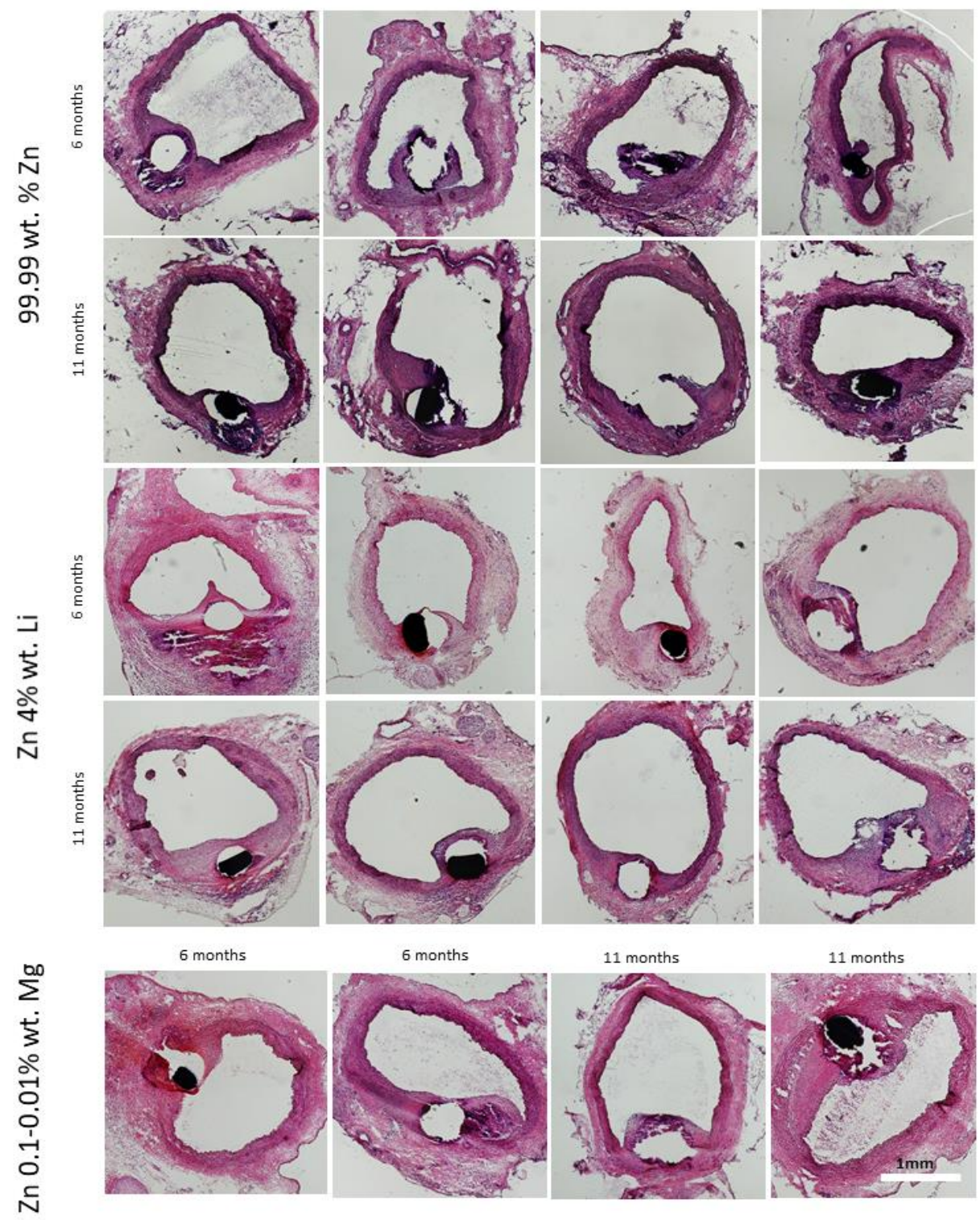

Fig.2-3 H\&E staining, 4 representative sample cross-sections of each implant at 6 and 11 month residence. Images are taken at 100X normal magnification and scale bar is approximately $1 \mathrm{~mm}$. 
In order to properly characterize neointima formations, thin tissue sections must be prepared and stained for each sample. Hematoxylin and eosin is the most common, general histological stain and we have routinely used this stain to evaluate materials implanted in the rat model [15-18, 24, 25]. Fig.3 shows multiple representative cross sections of 6 and 11 month samples that have been stained with H\&E in order to visualize neointimal formations. Inflammation and macro-level cellular responses can be readily identified at high magnification in these H\&E images, which we have demonstrated elsewhere $[18,25]$. Considering the presence and distribution of cellular type and inflammatory responses directly influence neointimal formations and contribute to the compatibility of intraluminal implants [23], a reliable and straightforward workflow of neointimal morphometric characteristics can be used to describe the overall cellular response to the material. To make measurements that describe the neointimal character, a VVG stain is more appropriate because this stain can be used to distinguish the different layers of arterial tissue.
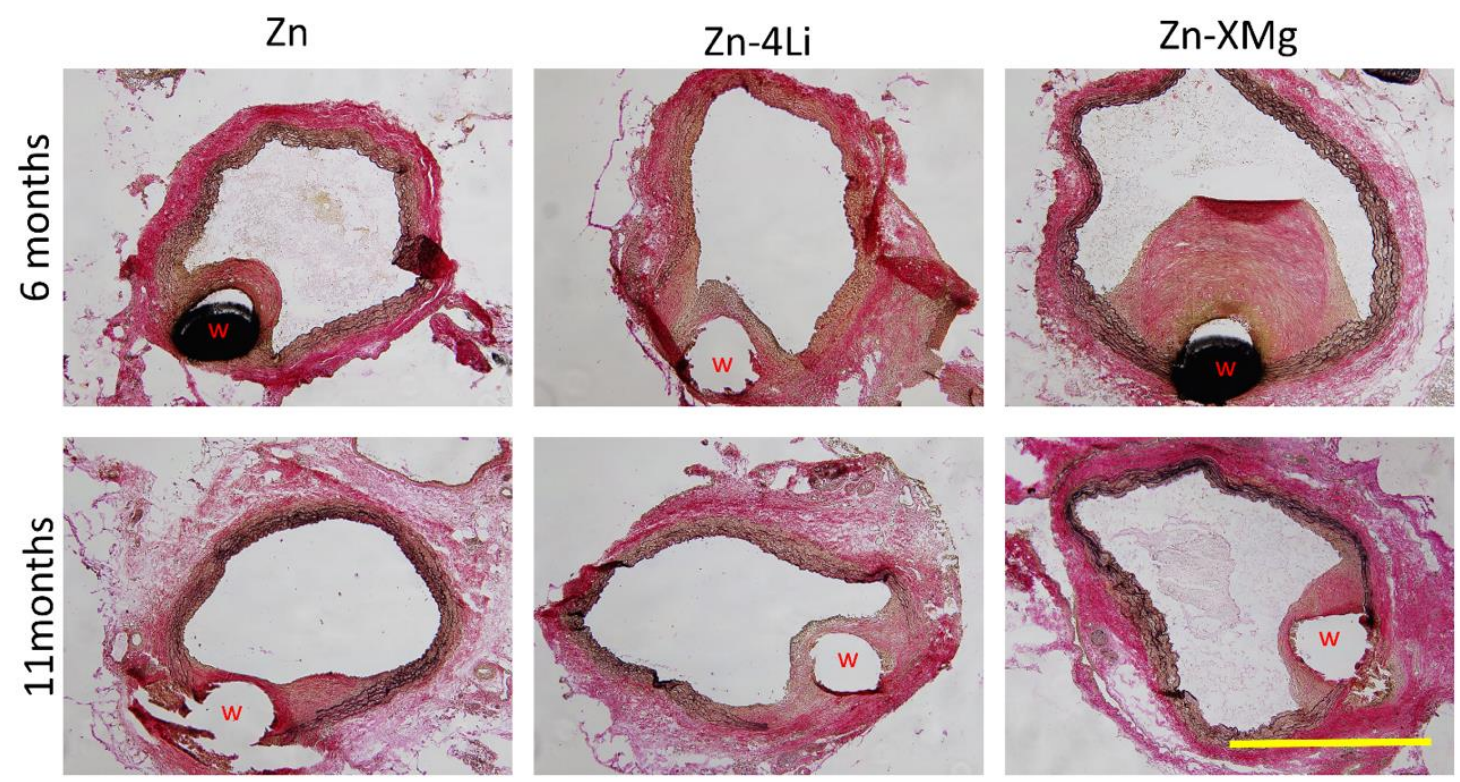

Fig.2-4 VVG staining, 1 representative sample at each time point for the alloys investigated. "W" denotes wire position. Scale bar is approximately $1 \mathrm{~mm}$ 
The elastic fibers (black) provide anatomical landmarks for NA and BNL quantifications, which are also identifiable but far less prominent on an H\&E stain. Fig.4 shows a representative VVG stain of each implanted alloy at 6 and 11 month time points. A general progression of Type $1 \mathrm{~A}$ to $1 \mathrm{~B}$ formation can be seen for the $\mathrm{Zn}$ wire. The $\mathrm{Zn}-4 \mathrm{Li}$ specimens generally display increased intimal activation away from the implant, with a reduction of elastic fibers near the implant. For Zn-XMg wires, large Type 2A and 2B formations are often seen at both time points, with a high variation in neointimal responses.

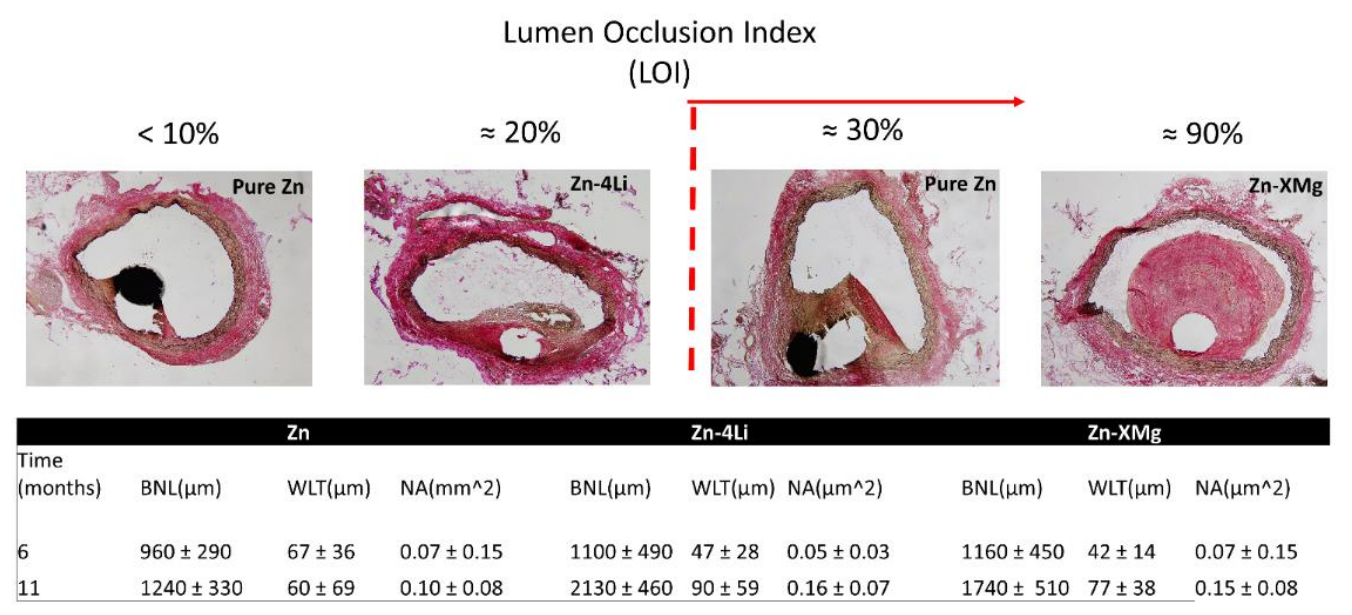

Fig.2-5 VVG staining of samples at indicated lumen obstruction index (LOI). Failure threshold is shown at $30 \%$. Tabulated morphometrics for the three alloy systems investigate at 6 and 11 months.

\subsubsection{Identification of optimal performance}

In order to quantify the direct success or failure of candidate materials, a lumen obstruction index was developed to describe negative histomorphometric appearances.

To obtain the lumen obstruction index of a sample: 


$$
\mathrm{LOI}=\left[\frac{\mathrm{WLT}}{\mathrm{BNL}-\Phi}\right] \times 100 \%
$$

where WLT is the wire to lumen thickness, BNL is the base neointimal length, and $\Phi$ is the nominal wire diameter, here as $250 \mu \mathrm{m}$. Presumably, a native artery that lacks a wire implant and has normal intima anatomy would have an LOI of $0 \%$. An index of $30 \%$ was set as the cutoff for degradable material success. This cutoff was based on the largest index measured for a pure $\mathrm{Zn}$ wire implant, out of 60 observations. Visual depiction of the failure index is presented in Fig.2-5, with the cutoff (dashed red line) identifying the failed pure $\mathrm{Zn}$ neointima. Out of all observations at both time points, one $\mathrm{Zn}$ implant at 6 months and three $\mathrm{Zn}-\mathrm{XMg}$ implants at 6 months exceeded the failure threshold. These implants were not included in the subsequent statistical analyses.

Fig.6 shows measurements from histological sections for samples at the two long-term evaluation time-points. At 6 months, no statistical differences exist between the $\mathrm{Zn}$ reference and Zn-4Li / Zn-XMg alloys for any metric. Additionally, the average WLT for $\mathrm{Zn}$ and the Zn-4Li/Zn-XMg alloys appears modest $(67 \pm 36 \mu \mathrm{m}$ vs $47 \pm 28 \mu \mathrm{m} / 42 \pm 14$ $\mu \mathrm{m}$ respectively). All three failed $\mathrm{Zn}-\mathrm{XMg}$ implants and the one failed $\mathrm{Zn}$ implant occurred at 6 months, indicating this time point could be useful as the minimum to observe failure. For the 11-month implants, all metrics for the alloys were significantly increased relative to the pure $\mathrm{Zn}$ reference material. WLT for the $\mathrm{Zn}-4 \mathrm{Li}$ and $\mathrm{Zn}-\mathrm{XMg}$ systems $(90 \pm 59 \mu \mathrm{m}$ and $77 \pm 38 \mu \mathrm{m})$ is significantly higher than for $\mathrm{Zn}, 60 \pm 69 \mu \mathrm{m}$ at 

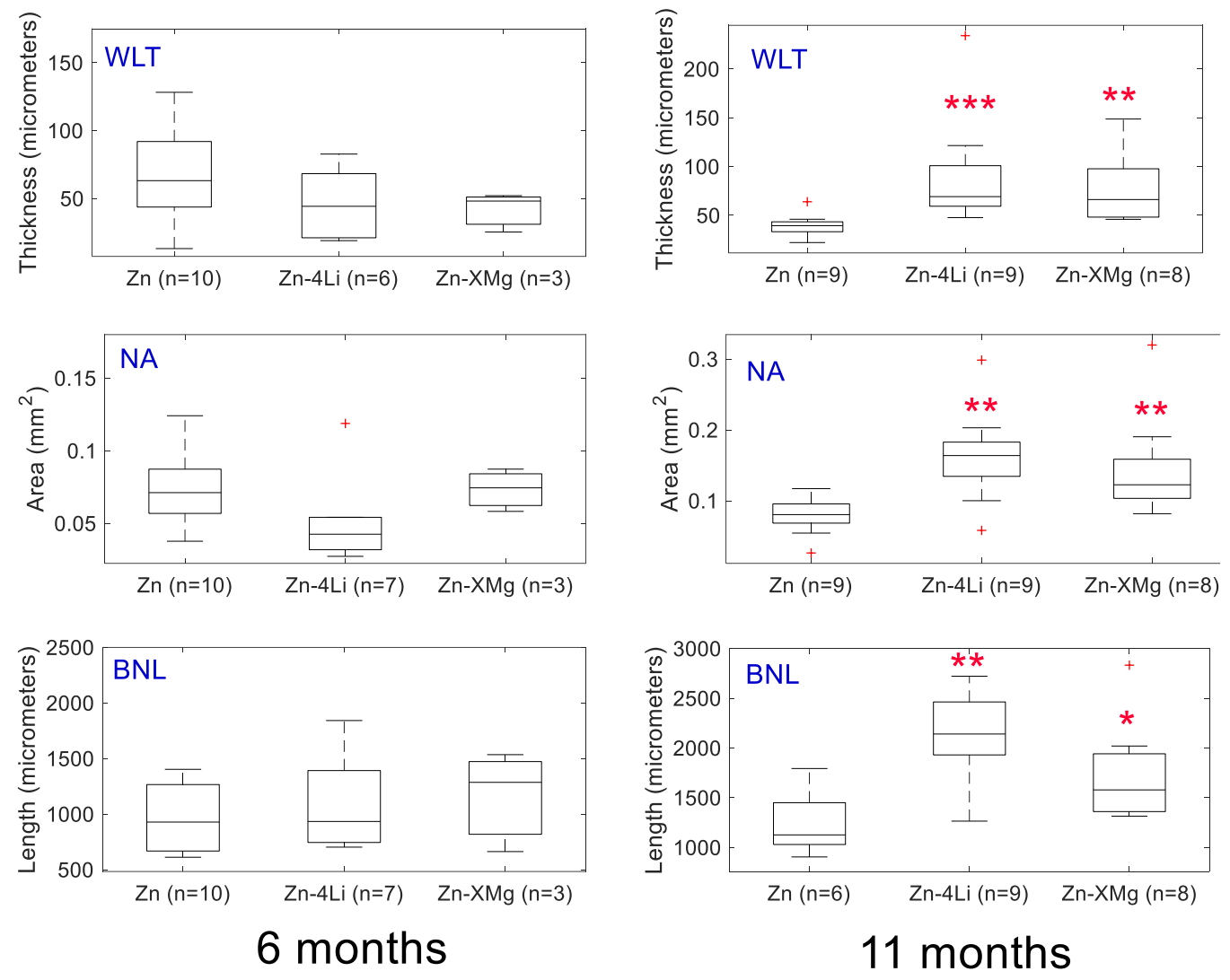

Fig.2-6 Boxplot representation of metrics at each time point, for each alloy system. The upper and lower quartiles (25\%-75\%) are within the boxed region, $0 \%$ and $100 \%$ quantiles within the whiskers, with red ' + ' as computed outliers. Significance was determined by a Wilcoxon rank sum test, with $p$ values in respect to the reference zinc sample at each time point. "*" $p<0.05$, "**" $\mathrm{p}<0.005, " * * * " \mathrm{p}<0.0005$.

11 months. Zn WLT appears unchanged from 6 to 11 months, while this metric appears to have increased markedly for $\mathrm{Zn}-4 \mathrm{Li}$ and $\mathrm{Zn}-\mathrm{XMg}$ implants.

Neointima thickness is commonly used to gauge arterial responses to stenting due to its correlation with stent failure and luminal area reduction [26-28]. In the present study, neointima thickness progression measured by WLT appears to be a reliable metric in 
determining compatibility. Furthermore, when divided by the length of neointimal activation (BNL) it can serve as an indicator of negative remodeling (closer to $100 \%$ indicates excessive protrusion within the lumen). The area of the neointima (NA) is important, but does not provide enough information to adequately describe performance of the material. For instance, two implants can have similar NA measurements but dramatically different failure indices. Therefore, NA is not recommended as a standalone metric for compatibility, but as a complementary evaluation.

Although a failure threshold of $30 \%$ is recommended, neointimal formation values greater than $20 \%$ should denote poor performance. Although none of the Zn-4Li implants met the failure threshold, multiple instances of increased WLT with failure indexes close to $20 \%$ (data not shown) suggest a reduction in compatibility for the $\mathrm{Zn}-4 \mathrm{Li}$ wires relative to pure $\mathrm{Zn}$.

\section{Zinc tissue quantification}

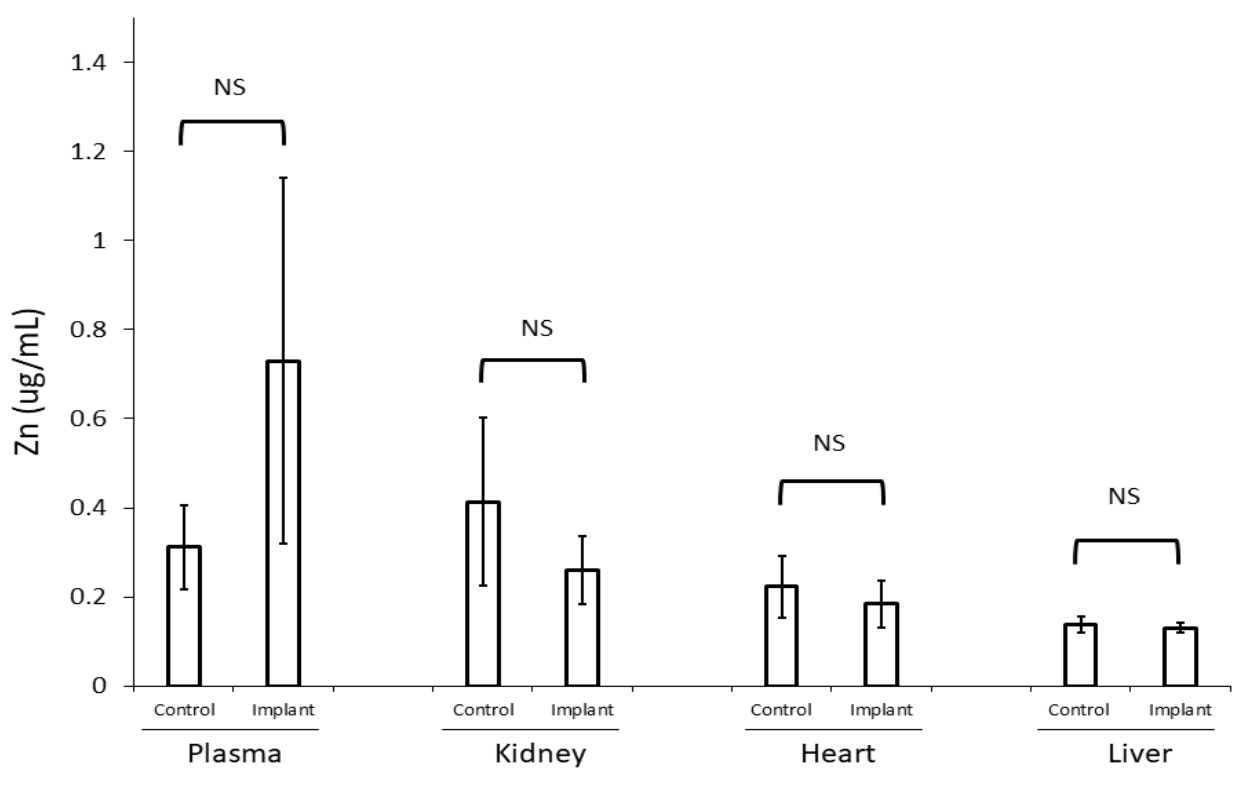


Fig.2-7 Zn Organ quantification by a flourometric assay. Bar graphs represent mean and error bars are \pm standard deviation of the samples. Minimum $n=3$ for each condition, significance was determined by a two tailed students t test, "NS" not significant

An organ analysis can also be performed on each animal, as shown in Fig.2-7. This analysis demonstrated a slight increase in plasma bearing $\mathrm{Zn}$, with no accumulation of zinc in the heart, liver, or kidney. This finding suggests that zinc removed from the implant is safely transported and excreted from the body.

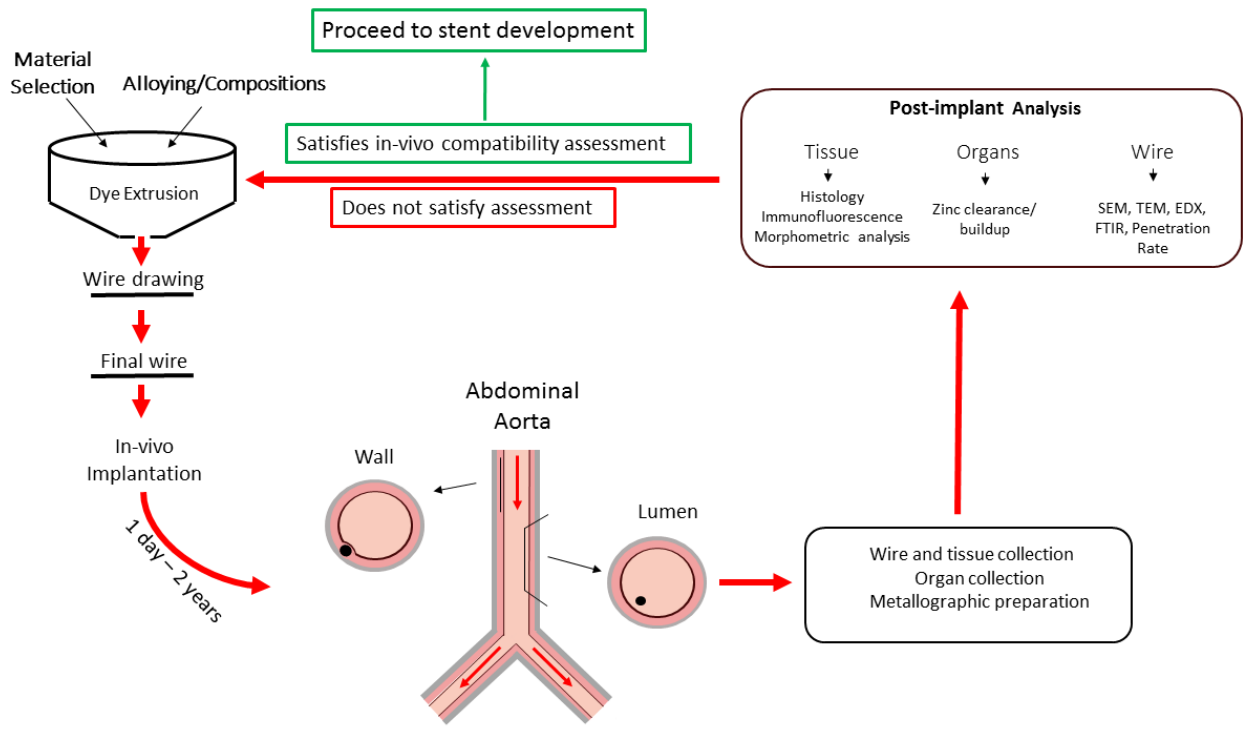

Fig.2-8 Workflow of wire model showing alloy development and biocompatibility testing 
The wide variety of $\mathrm{Zn}$-alloy systems that can serve in degradable cardiovascular stent applications has opened exciting possibilities for a number of candidate materials. The most appropriate approach for assessing the biocompatibility of degradable metals remains an important and open question for materials selection. Here, we present a robust workflow, summarized in Fig.2-8, for the testing of degradable metal candidates. Candidate materials are designed, extruded into rods, drawn to $\phi=0.25 \mathrm{~mm}$ wire, and characterized for mechanical and microstructure properties. The wires are then implanted into the lumen of Sprague Dawley rats and allowed to maintain residence for up to approximately $1-2$ years. Implants are then collected and histologically processed for biocompatibility assessment. Based upon quantification of metrics as outlined in the present contribution, informed decisions related to alloy design are iterated into the workflow, or selected materials can progress to stent testing in large animals. A summary of these proposed metric values are given below.

Table 2-1: Summary of the proposed biocompatibility values given by the wire model

\begin{tabular}{c|c}
\multicolumn{1}{c|}{ Metrics } & Biocompatibility standards (6-months) \\
\hline $\begin{array}{c}\text { Lumen obstruction index } \\
\text { (LOI) }\end{array}$ & $\leq 30 \%$ \\
Wire to lumen thickness \\
(WLT) \\
Neointima Type
\end{tabular}

Based upon the metrics developed here, pure $\mathrm{Zn}$ elicited the best biocompatibility of the three evaluated materials. An excellent biocompatibility response for pure $\mathrm{Zn}$ in the wire model is consistent with what was reported for pure zinc stents implanted within the 
rabbit abdominal aorta [19]. Therefore, the metrics we have developed based on implant materials in wire geometry may be predictive for the biocompatibility of stents deployed in large animals. Zn-4Li exhibited a reduction in biocompatibility at 11 months relative to pure $\mathrm{Zn}$ due to the progression of its WLT. Although no Zn-4Li wires incurred a LOI > $30 \%$, numerous implants incurred elevated LOI measurements above $10 \%$. The present finding of a non-obstructive neointima is consistent with previous preliminary observations of $\mathrm{Zn}-4 \mathrm{Li}$ wires made by our group [17].

$\mathrm{Zn}-\mathrm{XMg}$ performed the worst of the three implanted materials, based on three failures at 6 months, as determined by LOI, and a significant increase of WLT at 11 months relative to pure $\mathrm{Zn}$. This reduction in compatibility was also reported previously, and may be due to an increase of inflammation in $\mathrm{Zn}-\mathrm{XMg}$ implants [16]. Due to the well-known compatibility of $\mathrm{Mg}$, the authors speculate that the performance reduction was unrelated to the inclusion of Mg directly. Rather, the worsened response may have been caused by changes in corrosion behavior or the generation of intermetallics of reduced biocompatibility. These factors gave rise to Type $2 \mathrm{~A}$ and $2 \mathrm{~B}$ neointima formations, even within the same sample (not shown), confirming our earlier work highlighting the importance of corrosion behavior for regulating biological responses[18].

\subsection{Conclusions}

We conclude that:

Biocompatibility can be reliably determined using the rodent wire implant model

Six months is the minimum viable time point to confidently determine biocompatibility

Quantifications of neointima characteristics, including wire to lumen thickness and base neointima length, can describe the overall performance of degradable implants. 
A reduction in biocompatibility relative to pure zinc is seen for the $\mathrm{Li}$ and $\mathrm{Mg}$ alloy systems.

\subsection{Acknowledgments}

U.S. National Institute of Health (Grant \#R21EB024034-01A1) is acknowledged for funding this work. Roger J. Guillory II was supported by the National Science Foundation Graduate Research Fellowship Program.

\subsection{References}

[1] P.K. Bowen, E.R. Shearier, S. Zhao, R.J. Guillory, F. Zhao, J. Goldman, J.W. Drelich, Adv. Healthcare Mater. 5 1121-1140.

[2] E. Mostaed, M. Sikora-Jasinska, J.W. Drelich, M. Vedani, Acta biomater. 71 (2018) 1-23.

[3] G. Katarivas Levy, J. Goldman, E. Aghion, Metals 7 (2017) 402.

[4] J. Ma, N. Zhao, D. Zhu, ACS Biomater. Sci. Eng. 1 (2015) 1174-1182.

[5] E.R. Shearier, P.K. Bowen, W. He, A. Drelich, J. Drelich, J. Goldman, F. Zhao, ACS Biomater. Sci. Eng. 2 (2016) 634-642.

[6] D. Zhu, Y. Su, M.L. Young, J. Ma, Y. Zheng, L. Tang, ACS Appl. Mater. Interfaces 9 (2017) 27453-27461.

[7] J. Kubásek, D. Vojtěch, E. Jablonská, I. Pospíšilová, J. Lipov, T. Ruml, Mater. Sci. Eng., C 58 (2016) 24-35.

[8] H. Li, X. Xie, Y. Zheng, Y. Cong, F. Zhou, K. Qiu, X. Wang, S. Chen, L. Huang, L. Tian, Sci. Rep. 5 (2015) 10719.

[9] Z. Tang, H. Huang, J. Niu, L. Zhang, H. Zhang, J. Pei, J. Tan, G. Yuan, Mater. Des. 117 (2017) 84-94.

[10] Z. Tang, J. Niu, H. Huang, H. Zhang, J. Pei, J. Ou, G. Yuan, J. Mech. Behav. Biomed. Mater. 72 (2017) 182-191.

[11] P. Libby, G.K. Hansson, Circ. Res. 116 (2015) 307-311.

[12] T. Inoue, K. Croce, T. Morooka, M. Sakuma, K. Node, D.I. Simon, J. Am. Coll. Cardiol. 4 (2011) 1057-1066.

[13] Y. Chen, Z. Xu, C. Smith, J. Sankar, Acta biomater. 10 (2014) 4561-4573. 
[14] M. Peuster, C. Hesse, T. Schloo, C. Fink, P. Beerbaum, C. von Schnakenburg, Biomaterials 27 (2006) 4955-4962.

[15] D. Pierson, J. Edick, A. Tauscher, E. Pokorney, P. Bowen, J. Gelbaugh, J. Stinson, H. Getty, C.H. Lee, J. Drelich, J. Biomed. Mater. Res., Part B 100 (2012) 58-67.

[16] H. Jin, S. Zhao, R. Guillory, P.K. Bowen, Z. Yin, A. Griebel, J. Schaffer, E.J. Earley, J. Goldman, J.W. Drelich, Mater. Sci. Eng., C 84 (2018) 67-79.

[17] S. Zhao, J.-M. Seitz, R. Eifler, H.J. Maier, R.J. Guillory II, E.J. Earley, A. Drelich, J. Goldman, J.W. Drelich, Mater. Sci. Eng., C 76 (2017) 301-312.

[18] R.J. Guillory, P.K. Bowen, S.P. Hopkins, E.R. Shearier, E.J. Earley, A.A. Gillette, E. Aghion, M. Bocks, J.W. Drelich, J. Goldman, ACS Biomater. Sci. Eng. 2 (2016) 2355-2364. [19] H. Yang, C. Wang, C. Liu, H. Chen, Y. Wu, J. Han, Z. Jia, W. Lin, D. Zhang, W. Li, Biomaterials 145 (2017) 92-105.

[20] M. Joner, A.V. Finn, A. Farb, E.K. Mont, F.D. Kolodgie, E. Ladich, R. Kutys, K. Skorija, H.K. Gold, R. Virmani, J. Am. Coll. Cardiol. 48 (2006) 193-202.

[21] A. Murata, D. Wallace-Bradley, A. Tellez, C. Alviar, M. Aboodi, A. Sheehy, L. Coleman, L. Perkins, G. Nakazawa, G. Mintz, J. Am. Coll. Cardiol. 3 (2010) 76-84.

[22] G. Nakazawa, A.V. Finn, M. Vorpahl, E.R. Ladich, F.D. Kolodgie, R. Virmani, J. Am. Coll. Cardiol. 57 (2011) 390-398.

[23] R. Kornowski, M.K. Hong, F.O. Tio, O. Bramwell, H. Wu, M. Leon, J. Am. Coll. Cardiol. 31 (1998) 224-230.

[24] A.A. Shomali, R.J. Guillory, D. Seguin, J. Goldman, J.W. Drelich, Surf. Innovations 5 (2017) 211-220.

[25] P.K. Bowen, R.J. Guillory II, E.R. Shearier, J.-M. Seitz, J. Drelich, M. Bocks, F. Zhao, J. Goldman, Mater. Sci. Eng., C 56 (2015) 467-472.

[26] A.W. Heldman, L. Cheng, G.M. Jenkins, P.F. Heller, D.-W. Kim, M. Ware Jr, C. Nater, R.H. Hruban, B. Rezai, B.S. Abella, Circulation 103 (2001) 2289-2295.

[27] R.S. Schwartz, K.C. Huber, J.G. Murphy, W.D. Edwards, A.R. Camrud, R.E. Vlietstra, D.R. Holmes, J. Am. Coll. Cardiol. 19 (1992) 267-274.

[28] S. Windecker, M. Haude, F.-J. Neumann, K. Stangl, B. Witzenbichler, T. Slagboom, M. Sabaté, J. Goicolea, P. Barragan, S. Cook, Circ.: Cardiovasc. Interventions 8 (2015) e001441. 


\section{Contributions of the surface characteristics of degradable zinc implants to the neointimal response and subsequent biocompatibility 56}

\subsection{Introduction}

Zinc ( $\mathrm{Zn})$-based metals are emerging materials for biodegradable cardiovascular devices ${ }^{1-}$ ${ }^{4}$. Although many questions remain, a number of recent preclinical studies have reported an encouraging efficacy for pure zinc and Zn alloy based stents ${ }^{5-7}$. Additionally, Zn-based metallic implants are steadily closing in on the mechanical benchmarks required for stenting human arteries ${ }^{7-9}$. However, in addition to mechanical strength and ductility, degradable Zn-based stents may require an engineered surface oxide film to initiate a relatively uniform corrosion and biocompatible host response in early stages of implanttissue interactions ${ }^{10-12}$. While there has been considerable progress in the development of novel Zn-based degradable alloys with improved mechanical and structural properties, the development of surface films has consistently lagged behind ${ }^{10,13}$. This is probably due to widely accepted standards for permanent stents related to smooth and electropolished surfaces, which may not apply directly to degradable metals.

A key feature of degradable $\mathrm{Zn}$ is an in vivo corrosion rate that is sufficient for device dissolution within 1-2 years ${ }^{11}$. Interaction with the host occurs at the $\mathrm{Zn}$ implant-tissue interface throughout the service lifetime, although the Zn-based substrate interface changes over time ${ }^{14}$. Continuous corrosion at the implant interface and the production of various biodegradation products elicit a host response that is sustained until the implant is fully degraded. It is well known that a change in the passivity or susceptibility to corrosion is determined largely by the surface film characteristics ${ }^{15-17}$. However, the ideal surface properties for degradable $\mathrm{Zn}$ materials remain an open question. Critically, a

\footnotetext{
5 This chapter contains material previously published in ACS Applied Materials and Interfaces

${ }^{6}$ References in this chapter follow ACS author guidelines
} 
surface that inadvertently provokes a rapid or non-uniform corrosion of the underlying degradable Zn substrate could result in localized metal dissolution and changes in $\mathrm{pH}$. These processes, if not controlled, may be harmful to surrounding cells, initiate inflammation and/or neointimal growth, and increase susceptibility of implant to stress corrosion cracking. In contrast, an improved biological response and mechanical performance could be imparted from engineered $\mathrm{Zn}$ surfaces that increase corrosion resistance and corrosion uniformity.

Conventional biostable stents are commonly surface treated by electropolishing (EP) to create a smooth surface finish with a thin, stable oxide layer that protects the underlying metal from corrosive attack ${ }^{18}$. Since degradable metals are designed to corrode, a thin EP surface layer may be highly sensitive to local variations in cells and tissue composition. In contrast to $\mathrm{EP}$, the anodization (AD) of metal surfaces may increase the stability of the surface layer and therefore reduce its sensitivity to non-uniform penetration from local variations in the biological milieu ${ }^{19,20}$. Both EP and AD processes are widely used in the biomedical industry as surface finishing treatments ${ }^{10,21}$. During EP, the surface material is removed by anodic dissolution leaving a smoother surface with reduced roughness and improved cleanability ${ }^{22}$. AD consists of an electrolytic passivation, which is used to increase the thickness of the natural oxide layer of the implant. Both EP and AD procedures have been reported to dramatically improve the biological performance of permanent ${ }^{23-26}$ and degradable implants.

The EP and AD protocols are well developed for inert implant materials such as stainless steel, titanium alloys and cobalt alloys. However, they are in their infancy for Zn-based implant materials. Here, we describe methods to exert control over the formation and uniformity of surface oxide films on Zn material surfaces using electropolishing and anodization processes. Zinc materials with engineered surfaces were characterized by electrochemical methods. They were then implanted into Sprague Dawley rats for 2-8 weeks in an effort to correlate neointimal responses to surface oxide film characteristics. We found that anodization of $\mathrm{Zn}$ implants, although not commonly applied to vascular stents, could benefit the biocompatibility of Zn-based stents if engineered appropriately. 


\subsection{Experimental}

\subsubsection{Surface preparation}

High purity $(99.99 \%) \mathrm{Zn}$ wires $(\mathrm{d}=0.25 \mathrm{~mm})$ and discs $(\mathrm{d}=8 \mathrm{~mm})$, ethanol $\left(\mathrm{C}_{2} \mathrm{H}_{5} \mathrm{OH}\right)$, butanol $\left(\mathrm{C}_{4} \mathrm{H}_{9} \mathrm{OH}\right)$, aluminum chloride hexahydrate $\left(\mathrm{AlCl}_{3} \cdot 6 \mathrm{H}_{2} \mathrm{O}\right)$, zinc chloride $\left(\mathrm{ZnCl}_{2}\right)$, oxalic acid $\left((\mathrm{COOH})_{2}\right)$, and industrial detergent $\left(\mathrm{Citranox}^{\circledR}\right)$ were purchased from Sigma Aldrich (St. Louis, MO, USA). The EP process was optimized bearing in mind criteria that provide reduced surface roughness and dimensional precision aimed to minimize thickness loss, which is of paramount importance for thin wires and stents. The electrolyte was designed based on the indications of ASTM E1558-09 standard ${ }^{27}$. Regarding anodization, the process parameters were selected to allow maximum surface coverage with minimum coating thickness. The study consisted of $\mathrm{Zn}$ wires and discs treated under a combination of different voltages, mixing speed, electrolyte concentration and treatment duration. The EP and AD sample surface finish was assessed by microscopic observations. Subsequently, the samples exhibiting desired surface properties were selected for in vitro and in vivo degradation study. $\mathrm{Zn}$ samples were cleaned with a detergent, then subjected to an ultrasonic cleaning process in pure acetone, followed by rinsing with deionized water. After that, the specimens were electropolished with an electrolytic solution comprised of $\mathrm{C}_{2} \mathrm{H}_{5} \mathrm{OH}(885 \mathrm{~mL}), \mathrm{C}_{4} \mathrm{H}_{9} \mathrm{OH}(100 \mathrm{~mL})$, $\mathrm{AlCl}_{3} \cdot 6 \mathrm{H}_{2} \mathrm{O}(109 \mathrm{~g}), \mathrm{ZnCl}_{2}(250 \mathrm{~g})$, and distilled water (120mL). A 20 x $20 \mathrm{~mm} 316 \mathrm{~L}$ stainless steel sheet was used as a cathode. The distance between the electrodes was $\sim 60$ $\mathrm{mm}$. The current was $0.45 \mathrm{~A}$, corresponding to a voltage of $\sim 26-28 \mathrm{~V}$, and the temperature was $\sim 21-23{ }^{\circ} \mathrm{C}$. The duration of the procedure was $\sim 90 \mathrm{~s}$. Throughout the course of electropolishing the solution was stirred with magnetic agitation to avoid waviness and streaks on the surface. Prior to anodization, $\mathrm{Zn}$ discs and wires were electropolished. The samples were submerged in $1 \mathrm{~L}$ of $0.5 \mathrm{M}(\mathrm{COOH})_{2} .(\mathrm{COOH})_{2}$ was selected as an electrolyte because of the oxalate ability to bind strongly to the metallic surface, thus improving the substrate corrosion resistance ${ }^{28}$. Anodization occurred for 1 min at room temperature using a 316L stainless steel cathode. The power supply was set to a current of $\sim 4 \mathrm{~A}$ corresponding to a voltage of $\sim 10 \mathrm{~V}$. During anodization, the 
electrolyte solution was constantly stirred with magnetic agitation to reduce the thickness of the double layer at the metal-electrode interface to obtain uniform local current densities on the $\mathrm{Zn}$ electrode. All samples were cleaned in an ultrasonically stirred acetone bath for $3 \mathrm{~min}$ and in a $75 \%(\mathrm{v} / \mathrm{v})$ ethanol bath for $5 \mathrm{~min}$ after each stage of preparation.

\subsubsection{Surface characterization}

A FEI Philips XL 40 equipped with a tungsten filament and operated with an acceleration voltage of $15 \mathrm{kV}$ was used for scanning electron microscopy (SEM) analyses. Micrographs were acquired with a probe current in the range of $1 \cdot 10^{-10}$ to $1 \cdot 10^{-8} \mathrm{~mA}$. Xray photoelectron spectroscopy (XPS) characterization was carried out to determine the chemical composition of the EP and AD surfaces (PHI 5800 spectrometer, Physical Electronics U.S.A), with an incident angle of $45^{\circ}$ with respect to the surface, and a residual pressure of $5 \cdot 10^{-9}$ Torr. A survey spectrum was recorded using a standard magnesium X-ray source for survey spectra $(0-1400 \mathrm{eV})$ at $400 \mathrm{~W}$ to identify all elements present at the surface. High resolution spectra (HR) of C1s, O1s and $\mathrm{Zn} \mathrm{p} 3 / 2$ regions were recorded with a standard $\mathrm{Mg} \mathrm{K}_{\alpha} \mathrm{X}$-ray source $(1253.6 \mathrm{eV})$. The spectrometer work function was adjusted to give $284.8 \mathrm{eV}$ for the main C1s peak. Curve fittings were determined using the Gaussian-Lorentzian (80-20) function and a Shirley type background and performed using the software Multipack ${ }^{\circledR}$. Each peak was decomposed into Gaussian/Laurentian components, which have been attributed to the presence of oxides, hydroxides, and carbonates/water. C 1s peak fitting was carried out with following components: peak I C-C/C-H $(\sim 284.8 \mathrm{eV})$; peak II: -O-C=O- $(\sim 289.0 \mathrm{eV})$, and peak III: carbonate groups and/or adsorbed $\mathrm{CO}$ and $\mathrm{CO}_{2}\left(\sim 290.1 \mathrm{eV}^{29-31}\right)$. The oxygen peak was composed of contributions located at: peak I: $\sim 530 \mathrm{eV}$ (oxide); peak II $\sim 531.5 \mathrm{eV}$ (hydroxide); peak III: $\sim 533.5 \mathrm{eV}$ (carboxyl groups, carbon dioxide and/or adsorbed $\left.\mathrm{H}_{2} \mathrm{O}^{31,32}\right)$. For $\mathrm{Zn} 2 \mathrm{p}_{3 / 2}$, the subsequent peaks were identified: peak I ( 1021.8) metallic zinc, peak II ( 1022.) zinc oxide and peak III ( 1022.7) zinc hydroxide and/or carbonate. 


\subsubsection{In vitro degradation behavior}

The corrosion of EP and AD samples was studied in Hanks' solution supplemented with $0.35 \mathrm{~g} \mathrm{NaHCO}_{3} / \mathrm{L}$. The $\mathrm{pH}$ of the solution was adjusted to 7.4 using $1 \mathrm{M} \mathrm{NaOH}$ or $\mathrm{HCl}$. Open circuit potential (OCP), electrochemical impedance spectroscopy (EIS), and potentiodynamic polarization tests were performed to study the corrosion behavior of the investigated samples. The potentiodynamic test was performed using a conventional three-electrode cell (Princeton Applied Research Model K47) with a platinum counter electrode of $1 \mathrm{~cm}^{2}$ in surface area, a saturated calomel reference electrode and the prepared Zn-based working electrode. The OCP vs the SCE RE was monitored without applying any outside source for 3600 s until equilibrium was reached at the corrosion potential $\mathrm{E}_{\text {corr. }}$ A scan rate of $0.166 \mathrm{mV} / \mathrm{s}$, with an applied potential range of $1 \mathrm{~V}$, was used. The experiments were carried in an aerated environment at $37 \pm 1^{\circ} \mathrm{C}$. The solution was stirred with magnetic agitation ( 80 rounds/min) during the test. For each type of material, three specimens were tested using the same conditions. The electrodes were connected to a potentiostat (PARSAT4000, Princeton Applied Research, PA, USA) and monitored using the VersaStudio ${ }^{\circledR}$ software. The corrosion rates were obtained based on the calculated corrosion current density $\left(i_{\text {corr }}\right)$, using the following equation:

$$
\mathrm{CR}=3.27 \cdot 10^{-3} \frac{\mathrm{i}_{\text {corr } \mathrm{EW}}}{\rho} \quad \text { (Eq. 1) }
$$

where $C R$ is the corrosion rate $\left(\mathrm{mm} \mathrm{year}^{-1}\right), i_{\text {corr }}$ is the corrosion current density $\left(\mu \mathrm{A} \mathrm{cm}^{-2}\right)$ obtained on the base of potentiodynamic curves using Tafel extrapolation method, EW is the weight equivalent and $\rho$ is the material density $\left(\mathrm{g} \mathrm{cm}^{-3}\right)$.

Impedance spectra were separately collected from $100 \mathrm{kHz}$ to $0.1 \mathrm{~Hz}$ with $5 \mathrm{mV}$ perturbation amplitude. Because of reactive nature of zinc, no data points were collected below $0.1 \mathrm{~Hz}$. The OCP potential was recorded for 30 min prior to the EIS measurement to allow the interface to reach a steady state. EIS spectra were recorded after $1 \mathrm{~h}, 24 \mathrm{~h}$, $72 \mathrm{~h}, 120 \mathrm{~h}$ and $168 \mathrm{~h}$. The results were adjusted according to equivalent electric circuits using the software ZSimpWin ${ }^{\circledR}$. 
For static immersion tests, the Zn-based discs with $0.8 \mathrm{~cm}^{2}$ exposed surface area were immersed for 28 days in $40 \mathrm{~mL}$ of Hanks' solution. The whole volume of solution was changed every 7 days to keep the $\mathrm{pH}$ value close to 7.4 and to maintain conditions as constant as possible. The containers were stored in a controlled temperature $(\mathrm{T}=$ $37 \pm 1^{\circ} \mathrm{C}$ ), Three specimens were tested for each condition. The samples were subsequently washed with $200 \mathrm{~g} \mathrm{~L}^{-1}$ of chromium oxide in distilled water to remove corrosion products. The surface morphologies and chemical compositions before corrosion products removal were examined by SEM and energy dispersion spectrometry (EDS).

\subsubsection{In vivo wire implantation}

Adult female Sprague Dawley rats were purchased from Harlan Laboratories. Nine samples per treatment group were harvested over 2,4 , and 8 weeks ( 3 samples at each time point), using a surgical procedure described previously ${ }^{33}$. At the euthanization time point, the aorta containing the wire was dissected and excised, surrounded in polyfreeze cryo medium, snap frozen in liquid nitrogen, and stored in a -80 centigrade freezer until cryo-sectioning.

\subsubsection{Tissue preparation, histological, and morphometric analysis}

200x magnified H\&E images were uploaded to MetaMorph image analysis software. The thickest region of the neointimal growth was measured and recorded from cross sections taken from at least three different locations along the wire, spanning approximately 0.2 $\mathrm{mm}$. A detailed description of the methods for the morphometric analysis are described in our previous publication ${ }^{33}$.

\subsubsection{Statistical Analysis}

All data was uploaded into MATLAB with a statistics machine learning toolbox package. A Wilcoxon rank sum test was used to compare experimental groups. The lumen occlusion index was used as a cutoff to exclude outliers (LOI $\geq 30 \%)^{33}$. 


\subsection{Results}

\subsubsection{Surface characterization}
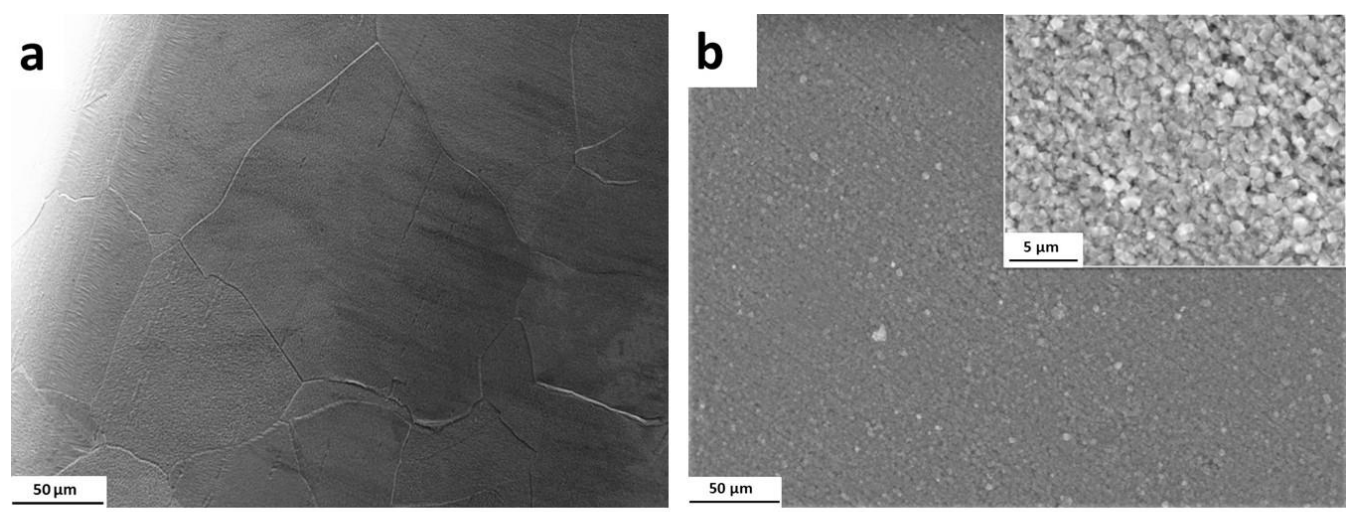

C

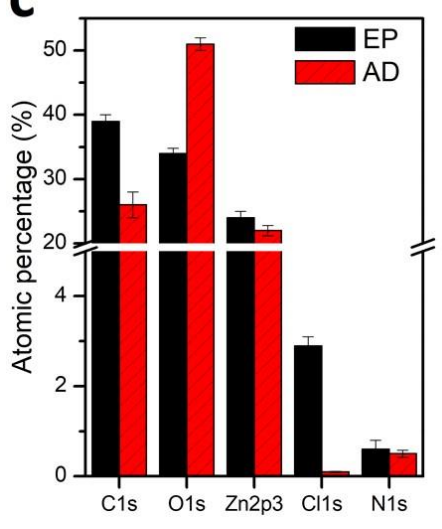

d

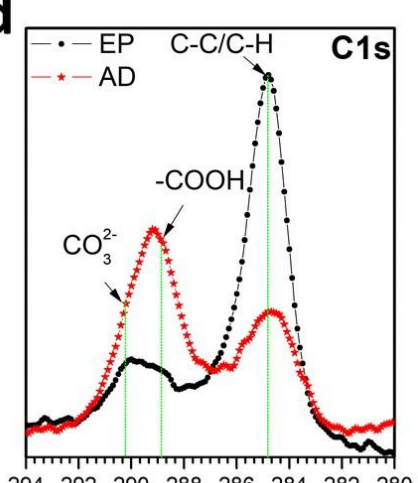

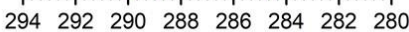

Binding energy (eV)

e

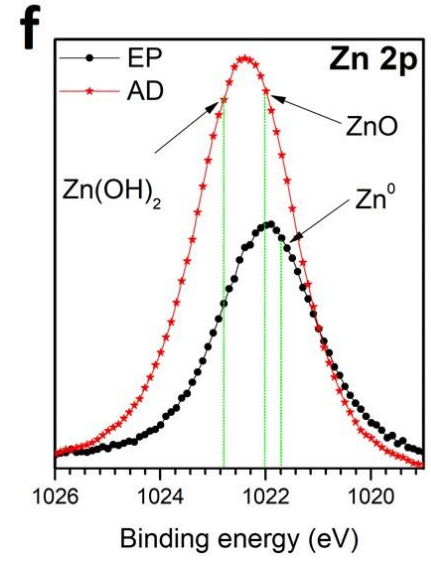

g

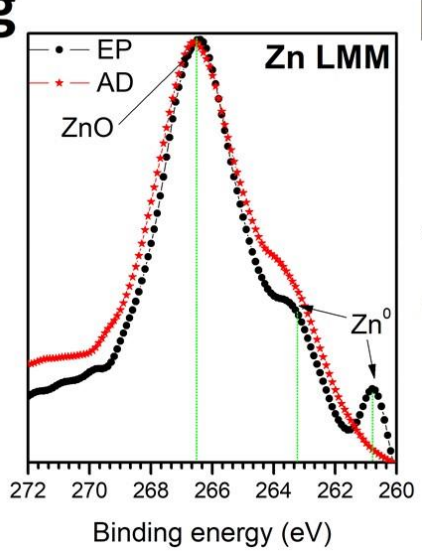

h
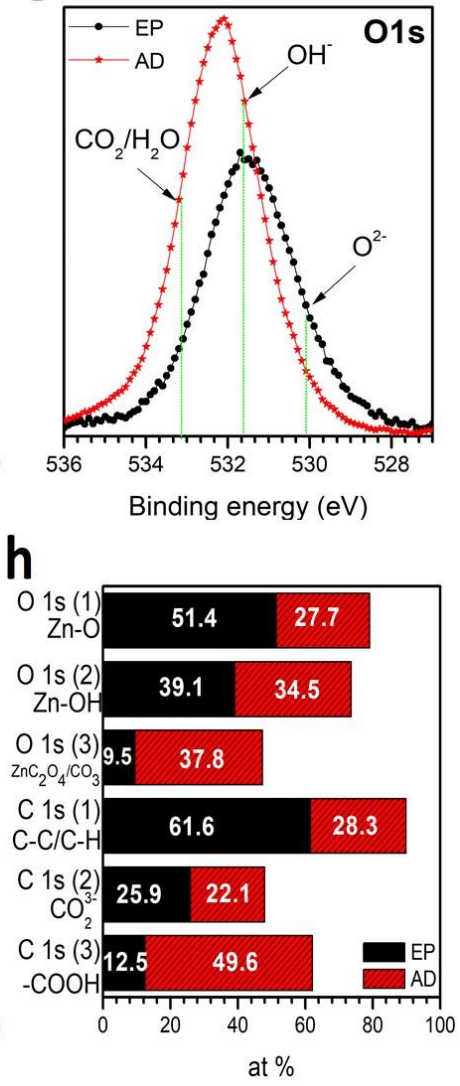
Fig.3-1. SEM micrographs of (a) EP and (b) AD samples, (c) Chemical composition of EP and AD Zn surfaces by XPS and overlay of XPS high resolution spectra and peak positions for (d) carbon - Cs1, (e) oxygen (O1s), (f) zinc (Zn $2 \mathrm{p} 3 / 2$ ) and ( $\mathrm{g}$ ) zinc auger (ZnLMM), (h) HR-XPS calculated values from the GaussLorentz fit for the $\mathrm{O} 1 \mathrm{~s}$ and Cs1 peaks.

SEM micrographs of the EP and AD surfaces are shown in Fig. 3-1. A smooth surface, similar to the industry standard of bare metal biostable stents, is evident on degradable $\mathrm{Zn}$ due to the EP process. In contrast, the Zn AD surface (Fig. 3-1b) is composed mainly of prismatic particles (with sizes in the range of $\sim 500-800 \mathrm{~nm}$ ), similar in morphology to what has been produced by other researchers following $\mathrm{Zn}$ anodization in oxalic acid ${ }^{34}$, 35. An ultra-thin oxide film ( $\sim 4 \mathrm{~nm})$ is formed on the EP surface (Fig. 3-2a), estimated using an XPS depth profile. The AD coating is relatively uniform, $\sim 6 \mu \mathrm{m}$ thick, and indicated by green two-headed arrow in Fig. 3-2c.

According to the XPS survey spectra, shown in Fig. 3-1c, Zn is present on both EP and $\mathrm{AD}$ surfaces. Non-metallic elements such as $\mathrm{C}, \mathrm{N}$, and $\mathrm{Cl}$ were detected on the surface of the both samples. Their presence is most likely a consequence of the surface preparation, as EP and AD treatments involve the use of different acids and solvents. Fig. 3-1d-g show the high-resolution scan data for C 1s, O 1s, Zn 2p $3 / 2$, and Zn LMM peaks. The amount of oxygen on the surface is significantly higher for the AD sample, implying a higher content of oxygen-containing compounds. Contamination from hydrocarbon compounds was observed on both surfaces. Its source is constituents of the ambient air. The presence of carbon has been observed on clean surfaces of most materials, even under moderately high vacuum conditions ${ }^{36}$. These carbon species are not involved in any chemical interaction with the underlying virgin surface species, as they are not very reactive by their inherent chemical nature ${ }^{37}$. The content of carbon was lower for $\mathrm{AD}$ samples as seen in Fig. 3-1c. In contrast, the air exposure of the EP surface resulted in rapid accumulation of $\mathrm{C}$ containing compounds, because chemically evolving clean metallic surfaces are more vulnerable to opportunistic carbon contamination ${ }^{37}$. The $\mathrm{C} 1 \mathrm{~s}$ 
HR spectra for EP samples reveal the main component as a C-C/C-H peak at $284.8 \mathrm{eV}$. The AD surface treatment resulted in a carbon presence, with the main peak appearing at a binding energy of $289.4 \mathrm{eV}$. This peak can be divided into two components: $\mathrm{C}$ in the carboxyl group (-O-C=O-) ascribed to $\mathrm{Zn}$ oxalate and/or carbonate groups and carbon at $290.1 \mathrm{eV}$. The peak at this binding energy is often reported as demonstrating evidence of bonding between carboxylic groups and the metallic surface ${ }^{38-40}$. The XPS Zn $2 \mathrm{p}_{3 / 2}$ spectra for EP show a peak at $1021.5 \mathrm{eV}$, corresponding to $\mathrm{Zn} / \mathrm{ZnO}$. This peak has a shoulder at higher binding energies, associated with the presence of $\mathrm{Zn}(\mathrm{OH})_{2}$ and/or $\mathrm{ZnCO}_{3}$. However, only a single component is present on the AD surface, which can be ascribed to zinc oxalate. The $\mathrm{Zn}$ Auger LMM peak revealed the presence of three peaks for EP sample. These peaks are related to metallic zinc and were not clear in the case of the AD surface. This might be due to the thicker zinc oxide film for the AD sample. The HR-O1s peak of samples has been fitted with three peaks: O1s(1) at a binding energy of $531.5 \mathrm{eV}$ assigned to $\mathrm{Zn}-\mathrm{O}, \mathrm{O} 1 \mathrm{~s}(2)$ at a binding energy of $531.6 \mathrm{eV}$ assigned to $\mathrm{Zn}-\mathrm{OH}$ and $\mathrm{O} 1 \mathrm{~s}$ (3) at a binding energy of $533.0 \mathrm{eV}$ assigned to $\mathrm{ZnC}_{2} \mathrm{O}_{4} / \mathrm{ZnCO}_{2}$. An analysis of the $\mathrm{O} 1 \mathrm{~s}$ spectra revealed an increase in $\mathrm{Zn}$ oxalate/carbonate for the $\mathrm{AD}$ sample relative to EP. The quantified concentrations associated with the $\mathrm{C}, \mathrm{O}$ and $\mathrm{Zn}$ peaks are presented in Fig. 1h. Considering the complex nature of oxidation/precipitation on $\mathrm{Zn}$ surfaces, the fitting of multiple $\mathrm{Zn}$ species is challenging. Unreliable interpretation can be produced for the speciation of the $\mathrm{Zn}$ surface chemical state due to many possible $\mathrm{Zn}$ species with overlapping binding energies. 


\subsubsection{Corrosion behavior}

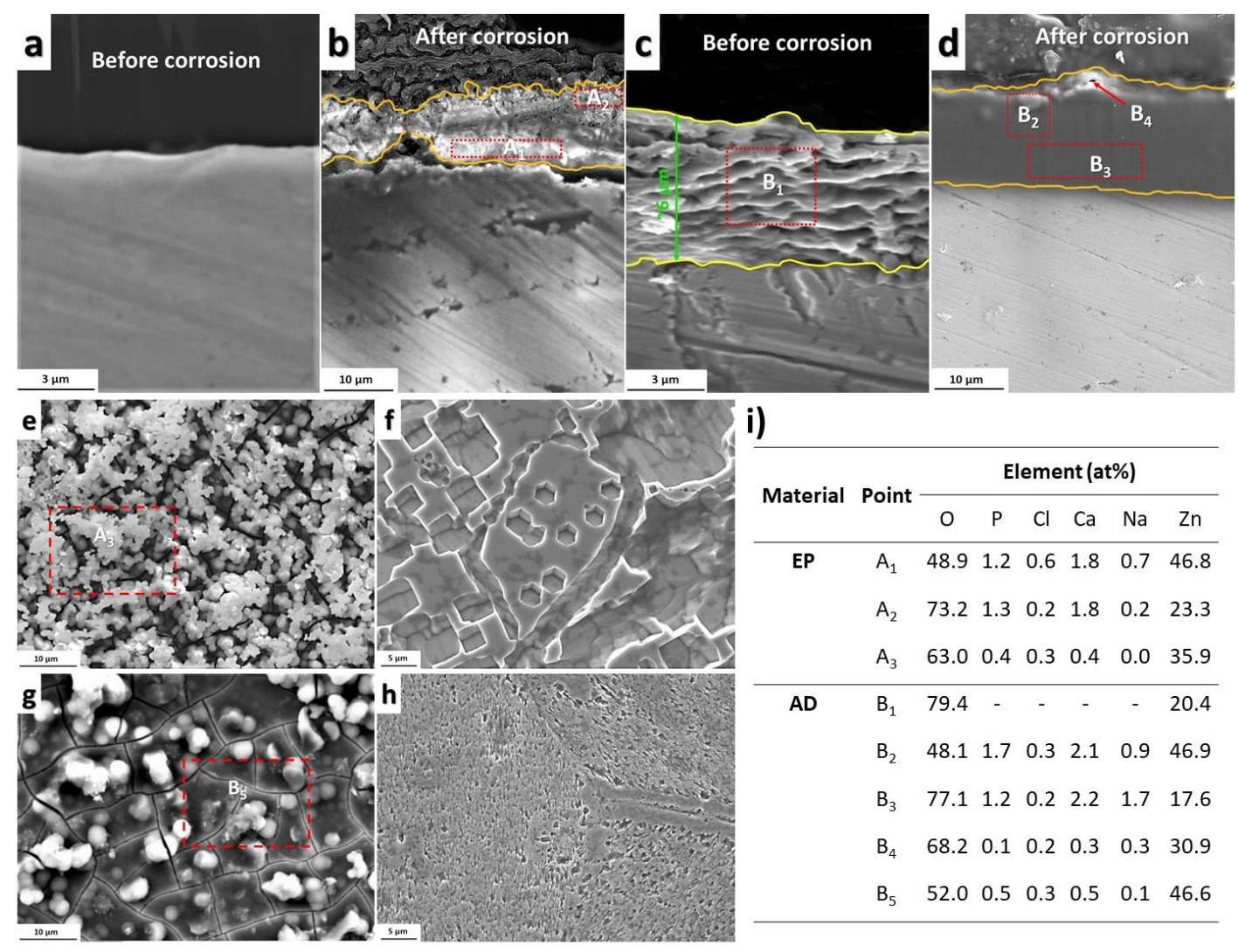

Fig.3-2. The typical cross-sectional morphologies of EP (a, b) and AD (c, d) before and after 28 days of exposure to Hanks' solution, the surface morphologies of corroded EP $(e, f)$ and $A D(g, h)$ before and after removing the corrosion products and EDS analyses (i) corresponding to the points and areas highlighted on micrographs. 
In order to clarify the corrosion mechanisms, the influence of the surface treatment on the polarization behavior of the $\mathrm{AD}$ and EP surfaces was investigated at $37^{\circ} \mathrm{C}$ in Hanks' solution by a morphological and chemical analysis before and after the corrosion experiment. Fig. 3-2 shows typical surface morphologies and cross sections of noncorroded and corroded samples. Cross sectional observation of corroded EP samples (Fig. 3-2 $\mathrm{a}, \mathrm{b})$ revealed the formation of a porous, non-uniform film with varied thickness from 2 to $10 \mu \mathrm{m}$. In contrast, the porous $\mathrm{AD}$ coating (Fig. 3-2c) underwent densification during the ongoing corrosion process. This may be due to blocked orifices in the anodic film from $\mathrm{Zn}$ degradation products, which formed a relatively dense barrier during the 28 days of exposure to Hanks' solution (Fig. 3-2d).

The effect of the surface treatment on the corrosion behavior can be more clearly assessed after removing degradation products. Signs of localized corrosion appear on the EP surface (Fig. 3-2f). Only small pits were observed on AD surface, indicating a surface attack that more than likely occurred during the deposition of the AD coating. EDS analyses of the corrosion products (which are listed in Fig. 3-2i), revealed the presence of $\mathrm{Zn}, \mathrm{Ca}, \mathrm{P}, \mathrm{O}, \mathrm{Cl}$ and $\mathrm{Na}$. Hanks' solution often produces degradation products that contain zinc oxides, hydroxides, carbonates, calcium phosphates, and carbonates ${ }^{41,42}$. The oxygen content may imply the precipitation of oxides and insoluble hydroxides. This is a result of the ionic reaction between $\mathrm{Zn}^{2+}$ and several acid radicals such as $\mathrm{Cl}^{-}, \mathrm{HCO}^{3-}, \mathrm{HPO}_{4}{ }^{2-}$, which are components of the testing solution. Corrosion products with atomic ratios of $\mathrm{Zn}: \mathrm{O}$ close to $1: 1$ are consistent with zinc oxide, a 1:3 $\mathrm{Zn}: \mathrm{O}$ ratio is typical for zinc carbonate $\left(\mathrm{ZnCO}_{3}\right)$. A ratio of $\mathrm{Zn}: \mathrm{O}$ exceeding 2 might suggest the presence of zinc hydroxide. Further, the presence of $\mathrm{ZnC}_{2} \mathrm{O}_{4}$ in anodic films (for samples before and after corrosion) is confirmed by a 1:4 Zn:O ratio. Additionally, different $\mathrm{Ca} / \mathrm{P}$ ratios on the degraded surfaces were found. The $\mathrm{Ca} / \mathrm{P}$ ratios of various calcium phosphate salts are the following: 1 for $\mathrm{CaHPO}_{4}\left(2 \mathrm{H}_{2} \mathrm{O}\right), 1.33$ for octacalcium phosphate (OCP), 1.5 for tri-calcium phosphate (TCP) and 1.67 for hydroxyapatite (HA). 
The electrochemical measurements provided further insights into the corrosion behavior of the surface treated samples. The respective polarization plots and their kinetic parameters are shown in Fig. 3-3a. According to the data, EP samples showed a minimum corrosion current density $\mathrm{I}_{\text {corr }}=17.17 \mu \mathrm{A}$ compared to $\mathrm{AD} \mathrm{I}_{\text {corr }}=20.12 \mu \mathrm{A}$. As seen in Fig. 3a, the cathodic current densities of EP and AD samples are similar. The anodic branches showed nearly the same trend. At potentials more positive than the Tafel range, the current density plateau was established at approximately $10^{-1} \mathrm{~A} \mathrm{~cm}^{-2}$. The anodic polarization curves of EP samples revealed a passivation-like region followed by an acceleration to a higher dissolution rate (Fig 3a). 

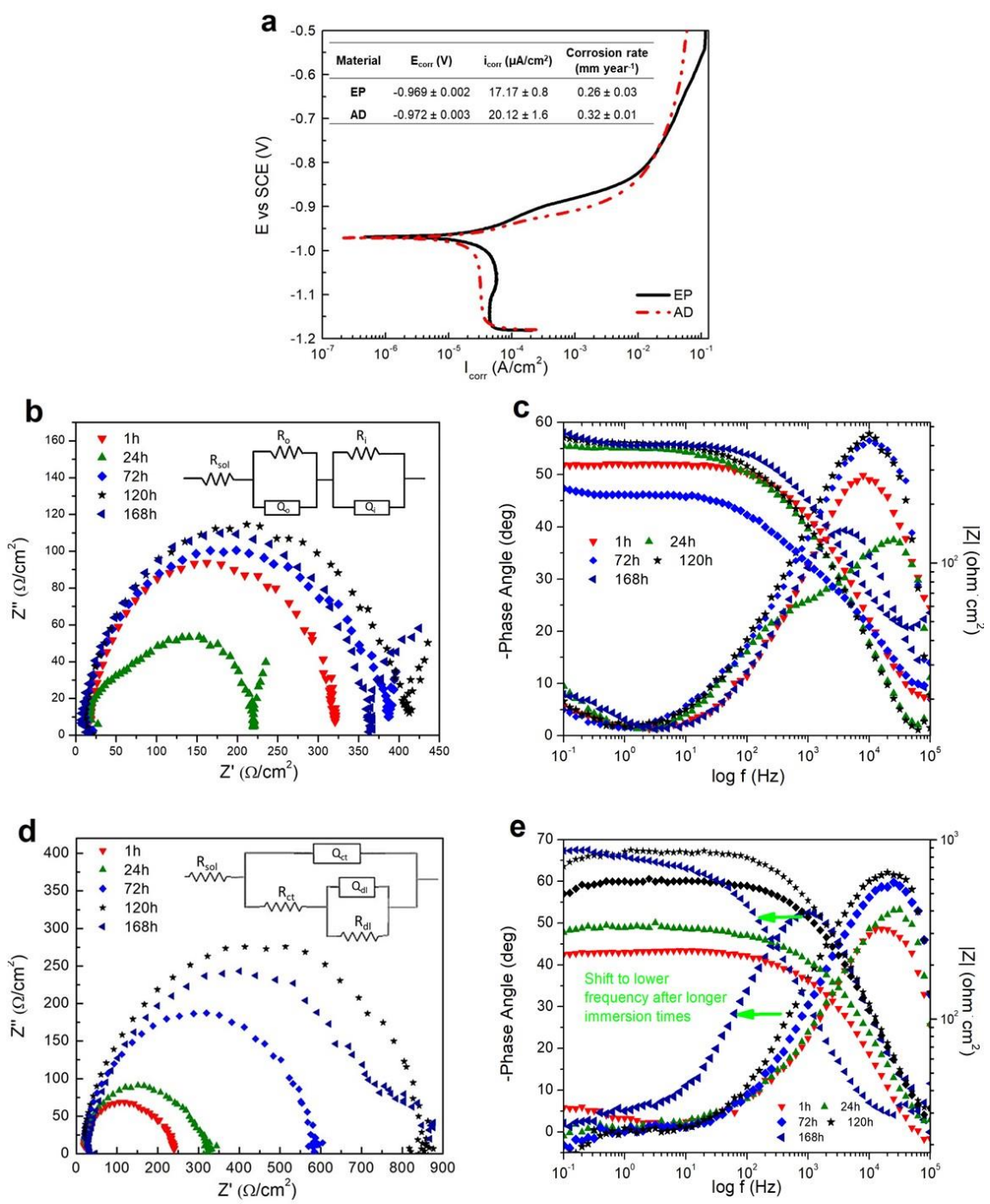

Fig.3-3. Electrochemical measurement plots for $A D$ and EP samples (a) potentiodynamic polarization curves (b) Nyquist and (c) Bode plots for EP samples, (d) Nyquist and (e) Bode plots for AD samples, after 1h, 24h, 72h, $120 \mathrm{~h}$, and $168 \mathrm{~h}$ of immersion in Hanks' solution

The evolution of the electrochemical behavior for the EP and AD was monitored by EIS as a function of time. Nyquist and Bode diagrams for both types of zinc surfaces, EP and 
AD are presented in Fig. 3-3 (b-d) for periods of exposure corresponding to 1h, 24h, 72h, $120 \mathrm{~h}$, and $168 \mathrm{~h}$. It is important to note that for 168 hours of exposure the impedances associated to the AD samples were superior to these of the EP substrate. Further, the results revealed that between 1 and 168 hours there was a large increase in the impedance of the AD samples (from $\sim 250 \Omega \cdot \mathrm{cm}^{2}$ to $\sim 900 \Omega \cdot \mathrm{cm}^{2}$, Fig. $3 \mathrm{~d}$ ), while that of the EP slightly decreased after 24 hours, reaching finally the value $400 \Omega \cdot \mathrm{cm}^{2}$ after $168 \mathrm{~h}$ (Fig. 3-3b). The results of the potentiodynamic tests refers to the sample surfaces after $1 \mathrm{~h}$ of exposure to the Hanks' solution. Accordingly, the results of EIS showed lower corrosion resistances for $\mathrm{AD}$ samples after 1h compared to EP (Fig. 3-3b, d). After 1h of exposure, a time constant to frequencies of around $10 \mathrm{kHz}$ is observed, likely due to charge transfer processes and charging of the double layer at the $\mathrm{Zn}$ surface and underneath the pores in the AD film. For 24 hours of exposure, the indication of another time constant at low frequencies associated to corrosion processes at the metallic substrate is seen on the Nyquist diagram (Fig. 3-3 b). This could be explained by the localized corrosion of the EP substrate and the formation of corrosion film. When comparing Bode plots of EP (Fig. 3-3c) and $\mathrm{AD}$ (Fig. 3-3e), both curves in impedance and phase angle plots of anodic coating showed a shift to a lower frequency. The AD Bode diagram corresponding to 168 hours of immersion test show a shoulder at frequencies around $1 \mathrm{kHz}$ and a peak at $1 \mathrm{~Hz}$ indicating a stable surface after the increasing dissolution of AD coating (Fig 3-3 e). Additionally, the part of AD coating under the influence of corrosive medium transformed into Zn-based oxides/carbonates/phosphates featuring low solubility and acting as a corrosion barrier. To quantify the changes in the impedance for EP and AD samples, the results presented in Fig. 3-3 were fitted using a circuit electric equivalent embedded in Nyquist plot (Fig. 3-3 b, d). The model providing an adequate fit of the EP data is shown in Fig 3-3b (inset). The EP/solution interface is interpreted as bi-layered corrosion product, made of an inner $\left(\mathrm{R}_{i}, \mathrm{Q}_{\mathrm{i}}\right)$ and an outer $\left(\mathrm{R}_{\mathrm{o}}, \mathrm{Q}_{\mathrm{o}}\right)$ layer. The $R_{\mathrm{i}}$ and $R_{\mathrm{o}}$ of EP displayed decreasing corrosion resistance within $24 \mathrm{~h}$, then increased gradually to 120 $\mathrm{h}$ and decreased again afterwards ${ }^{43-46}$. Both the capacitance and the resistance of the outer layer are high and the $\eta$ value indicate a non-perfect interface (0.7-0.9) (Table S1, supplementary materials), which can be attributed to a hydrated 
oxide/carbonate/phosphate layer. $\mathrm{R}_{\mathrm{i}}$ and $\mathrm{R}_{\mathrm{o}}$ decrease after the first $24 \mathrm{~h}$, indicating the disruption of the EP surface due to the corrosion attack. After 72h, the increase in resistance is observed suggesting the growth of the degradation products. The low $\mathrm{Q}_{\mathrm{i}}$ values after immersion times of $1 \mathrm{~h}, 24 \mathrm{~h}$ and $72 \mathrm{~h}$ are typical for porous films. Increasing $\mathrm{Q}_{\mathrm{i}}$ after $120 \mathrm{~h}$, suggest the formation of more stable, dense corrosion products layer. Accordingly, the corrosion resistance increases with degradation products formation and their accumulation on the EP sample surface. The high frequency arc on Nyquist plot was assigned to the charge transfer process, whereas the low frequency arc was allotted to the formation of unevenly distributed $\mathrm{Zn}$ corrosion products and species adsorbed on EP surface. The equivalent circuit depicted in Fig. 3-3d was used to fit spectra of AD sample. $R_{\text {sol }}$ was the solution resistance, $R_{\mathrm{ct}}$ and $Q_{\mathrm{dl}}$ represented the resistance of charge transfer and capacitance of the electrical double layer; $Q$ (constant phase element) was used here in place of a capacitor to compensate for the non-homogeneity of the system ${ }^{46,47}$. The $\mathrm{AD}$ film became homogeneous in terms of defects distribution, with lower number of micro-pores resulting in reduced roughness (and the actual surface area of the coating) leading to a decrease in $\mathrm{R}_{\text {sol }}$. The $\mathrm{R}_{\mathrm{ct}}$ value increases with progressing corrosion, suggesting the densification of the AD film. The diffusion resistance can be mainly attributed to the inner, denser layer of the coating. The reduction in $Q_{\mathrm{ct}}$ (reflecting the nature of the inner layer structure) comes from the thickening of the double layer, suggesting that corrosion products are formed at the surface/electrolyte interface. The rise of $\mathrm{R}_{\text {sol }}$ and $\mathrm{R}_{\mathrm{ct}}$ indicates the formation of a compact layer on the AD surface, and charge transfer reactions in the protective film, respectively, resulting in the densification of the $\mathrm{AD}$ layer and the homogenized, more corrosion resistant surface structure. The solution resistance $\left(R_{\text {sol }}\right)$ remained relatively stable during the entire experiment and the reproducibility was good for all three samples. 


\subsubsection{Histomorphometry and biocompatibility}

A representative image of the neointimal tissue that formed around the implants is shown for each condition in Fig.3-4. Thin, non-protruding neointima is observed for the AD implants, with a variable response to the EP implants.
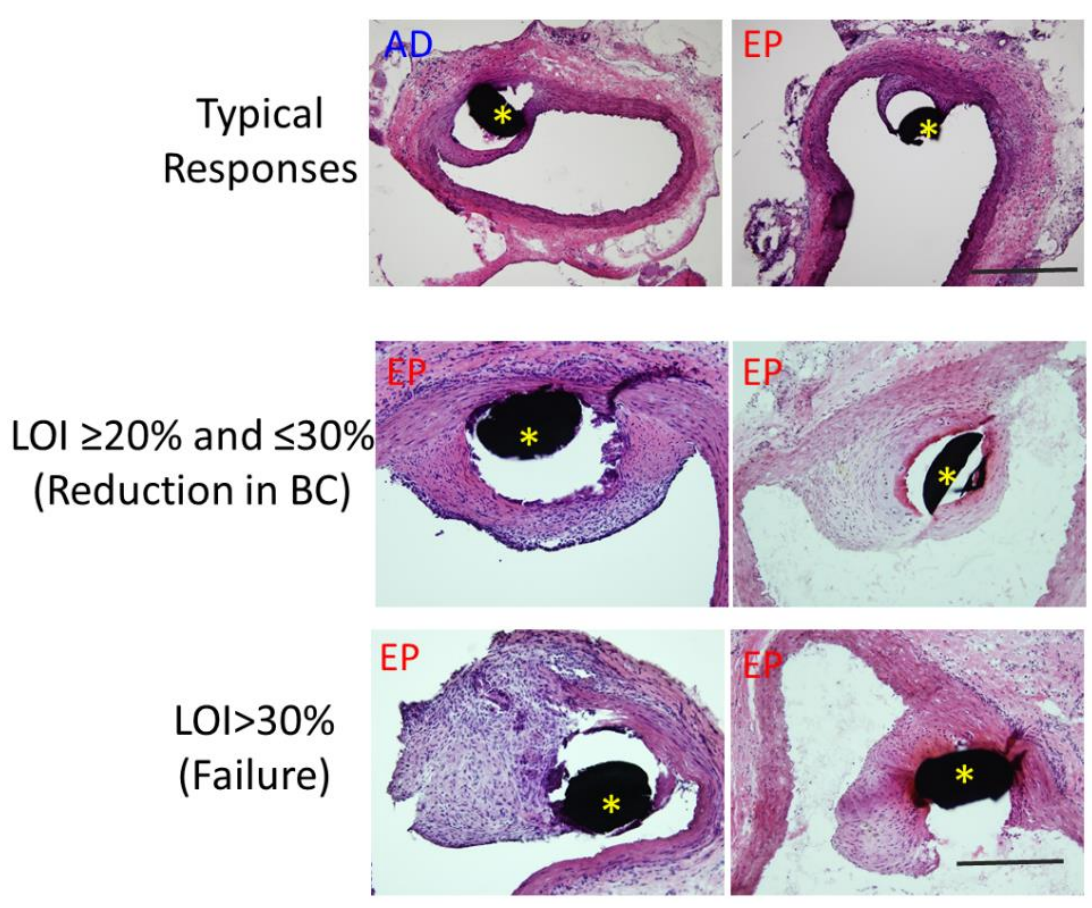

Fig.3-4. H\&E staining depicting typical (top row) and deleterious responses (bottom row)

The following equation was used to obtain the lumen occlusion index of a sample ${ }^{33}$,

$$
\mathrm{LOI}=\left[\frac{\mathrm{WLT}}{\mathrm{BNL}-\Phi}\right] \times 100 \%
$$

The lumen occlusion index (LOI), where WLT is the wire to lumen thickness, BNL is the base neointimal length (in micrometers), and $\Phi$ is the wire diameter (in micrometers). 

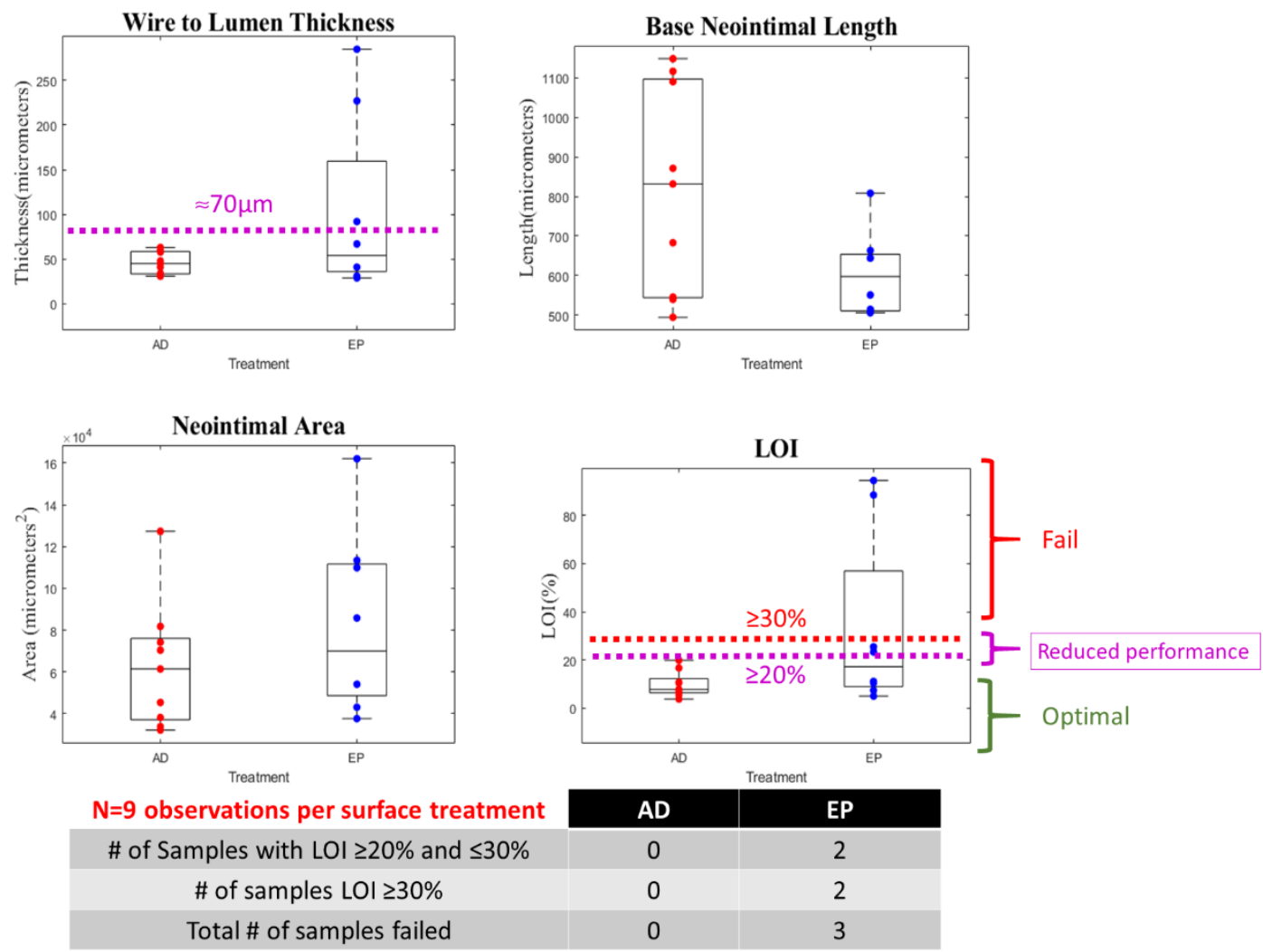

Fig.3-5. Quantification of in vivo histomorphometric analysis. Boxplots show the average with the boxed region ranging from $25 \%-75 \%$. Whiskers extend from $0 \%-100 \%$ with each dot representing one sample point.

While there were neither failures nor reductions in biocompatibility (BC) for any of the 9 $\mathrm{AD}$ treated samples, the EP condition produced 1 failure based on lack of endothelialization, 2 failures based on LOI, and 2 reductions in BC, as shown in Fig.5. The reductions in $\mathrm{BC}$ are defined as any neointimas with a $\mathrm{LOI} \geq 20 \%$ and $\leq 30 \%{ }^{33}$. Failures are defined as any samples with LOI of $\geq 30 \%$. The quantified histomorphometric analysis is shown in Fig. 3-5. One EP sample was excluded from the statistical analysis due to a lack of endothelialization. With this exclusion, no statistical difference between WLT $(45 \mu \mathrm{m} \pm 12 \mu \mathrm{m}$ vs $99 \mu \mathrm{m} \pm 100 \mu \mathrm{m})$, BNL $(813 \mu \mathrm{m} \pm 262 \mu \mathrm{m}$ vs 
$604 \mu \mathrm{m} \pm 105 \mu \mathrm{m})$ or NA $\left((6 \pm 8) \times 10^{4}\right.$ vs $\left.(8 \pm 5) \times 10^{4}\right)$ is observed. There is no difference between the two groups in terms of LOI $(10 \pm 5 \%$ vs $33 \pm 37 \%)$, although there is a trend towards significance $(\mathrm{p}=0.118)$.

\subsection{Discussion}

Although a degradable surface film is designed to rupture and eventually dissolve, it serves as the initial interface between the medical implant and biological environment. In this central position, the surface film regulates the host response and potentially direct long-term neointimal remodeling. Although the degradation products of zinc implants have been shown to inhibit harmful neointimal responses ${ }^{48}$, the surface film characteristics necessary to achieve optimal suppressive effects have not been explored in the scientific literature. Here, we have developed surface films through electropolishing and anodization processes with different characteristics to test the hypothesis that the surface film character of degradable zinc implants regulates neointimal responses. The character of $\mathrm{AD}$ and EP zinc surfaces was described based on surface features, corroded surface morphologies, cross sectional study, and electrochemical analyses. AD and EP surfaces prepared on $\mathrm{Zn}$ wires were then implanted into the abdominal aorta of Sprague Dawley rats for a direct comparison in terms of fundamental biocompatibility metrics.

We successfully prepared a smooth and defect-free surface (EP), similar to industry standard stents manufactured for clinical use. In marked contrast, the AD surface was rougher, with a bumpy nano-scale pattern and a thickness of about $\sim 6 \mu \mathrm{m}$ (Fig. 3-2c). The AD surface possessed a higher atomic percentage of oxygen and carbon, with potentially a presence of zinc carbonate and oxalate. In contrast, an ultra-thin oxide film was present on the EP sample. After in vitro corrosion, a porous corrosion layer was present on the EP sample, while a densified corrosion layer was recorded on the AD sample (Fig.3-2b, 2d). After removal of the corrosion products, large areas of localized corrosion were observed on the EP surface, while only small defects were visible on the AD surface that are ascribed to the surface attack during anodization process (Fig. 3-2f, 
2h). Although the EP and AD surfaces possessed similar corrosion potential values with similar corrosion rates (Fig.3-3a), the anodic region of the EP curve on Fig.3-3a displays a tendency towards dissolution when compared to $\mathrm{AD}$, revealing a slight passivation of the $\mathrm{AD}$ surface. The $\mathrm{AD}$ surface was resistant to corrosion overall, as shown by the initially high impedance values on the comparable Nyquist plots (Fig.3-3b, 3d) and the densified corrosion layer that formed on the AD sample. In contrast, the Nyquist and Bode plots of the EP condition revealed inferior corrosion resistance. Indeed, the development of localized corrosion was confirmed from the 28-day immersion in Hanks' solution (Fig.3-2f). 


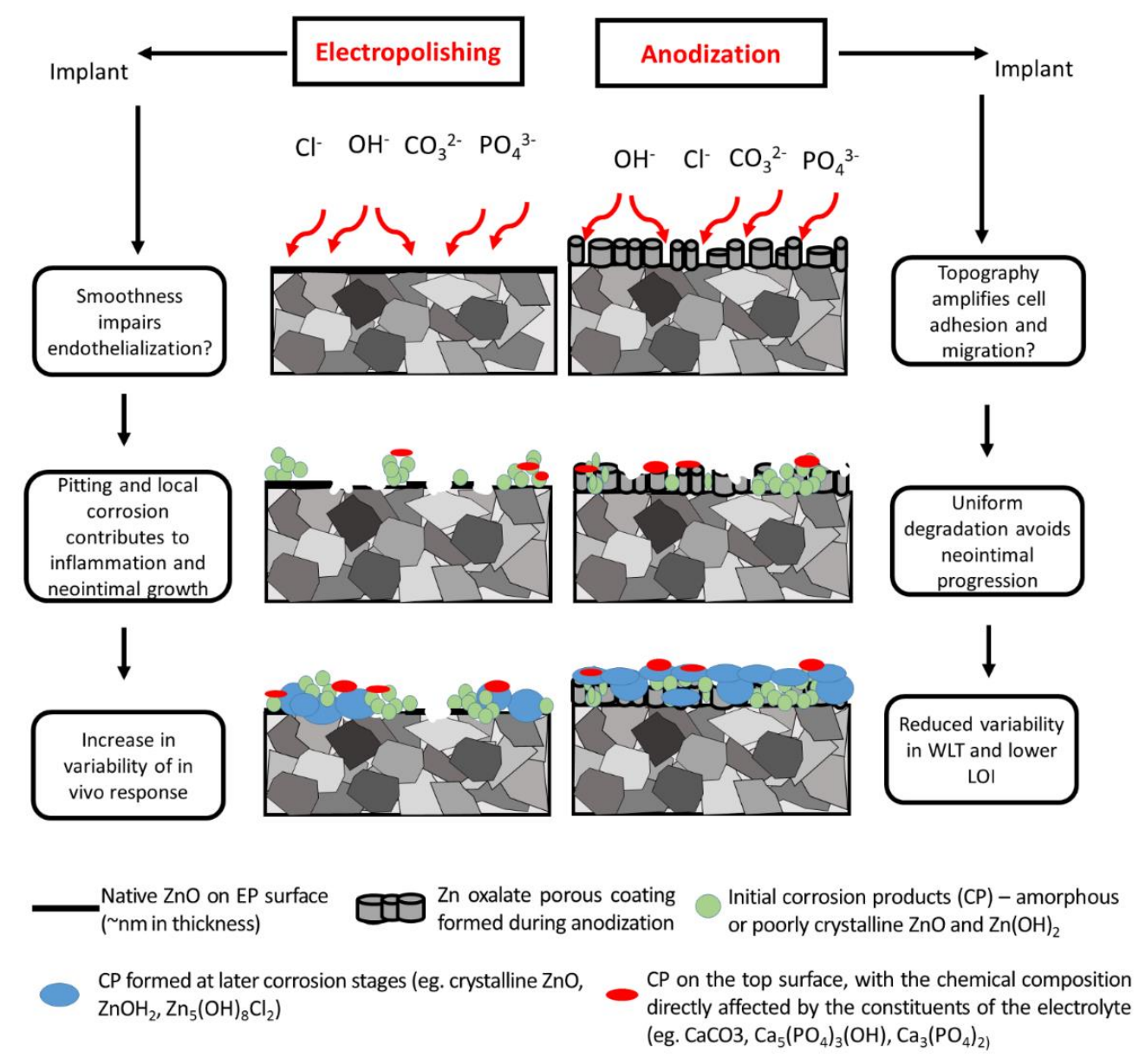

Fig.3-6. Schematic illustration of corrosion process of AD and EP samples.

The contributions of the prepared surface films to the corrosion behavior of the $\mathrm{Zn}$ materials can be described based on the presented in vitro pre and post corrosion analysis. With increasing exposure time, the reaction mechanism for carbonates, phosphates, and hydroxides is significantly slower, and mutual diffusion from the $\mathrm{Zn}$ substrate and Hanks' solution is reduced. Principal diffusion pathways are provided by the capillaries in the porous corrosion layers (and anodic film in the case of AD samples, Fig.3-6), where $\mathrm{Zn}$ ions diffuse from the bulk material toward the solution/surface interface. Ions present in the body fluid diffuse in the opposite direction (shown in Fig. 3-6). Thus, the degradation rates are reduced with corrosion product thickening and its densification. 
The surface oxide-hydroxide-phosphate-carbonate film on AD and EP samples cycle through a process of partial dissolution and re-precipitation in Hanks' solution. When the dissolution rate is larger than that of re-precipitation, ions are gradually released. When the metallic surfaces are covered with a thick and compact film, the corrosion resistance increases. However, the composition of this protective film might change even though these precipitates are macroscopically stable. The AD samples exhibited a higher corrosion resistance compared to EP, during longer exposure to Hanks' solution as shown by EIS measurements (Fig. 3-3). The Zn degradation precipitates blocked pores and capillaries in the $\mathrm{AD}$ film, protecting the $\mathrm{Zn}$ metal during longer exposures to physiological solution. In contrast, the protective film formed on EP samples was less compact, with varied thickness. Thus, it provided limited corrosion protection.

Since the noted differences in surface films influence corrosion behavior, we asked whether the different oxide film characteristics could provoke changes in the biocompatibility of zinc implants in the abdominal aorta of rats. Based on nine observations per treatment group, the $\mathrm{AD}$ surface produced stable, non-obstructing neointimal growth, while the EP preparations evoked a high rate of harmful responses (Fig. 3-4). Specifically, we observed two reductions in biocompatibility for the EP condition, and two outright failures in terms of LOI $\geq 30 \%$ (Fig.3-5). Another failure of one EP specimen was due to lack of endothelialization. The lack of roughness in the EP preparation vs. AD may explain the delay in endothelialization, as 3-dimensional surface features may aid in cell adhesion and migration ${ }^{49,50}$. In total, three out of the nine observations failed for the EP condition, and an additional two had a reduction in biocompatibility. In contrast, the nine $\mathrm{AD}$ specimens were free from failures or reductions in biocompatibility.

When evaluating the biocompatibility of vascular materials that are destined for human use, the numerical clustering of the $\mathrm{BC}$ metrics is an important consideration, even when the average response is acceptable from an application standpoint. The large spread in the tabulated data for the wire to lumen thickness (WLT) and lumen occlusion index (LOI) 
for EP specimens raises serious concerns, as it suggests that the in vivo response is nonuniform. The variability in biocompatibility responses could be a consequence of the non-uniform in- vitro corrosion behavior. Critically, corrosion product stability is reduced on the EP surface, leading to localized corrosion and pitting. The increase in substrate attack (apparent in Fig. 3-2) caused by a porous, loosely compacted corrosion layer that aids in solute diffusion to the metal-product interface could promote localized sites of inflammation along the wire that initiate neointimal growth and smooth muscle cell proliferation. Thus, increased variability from localized corrosion due to surface film character directly translates to impaired in vivo performance. Overall, the EP condition increased variability in the WLT metric, and produced a near significant increase in the LOI ( $\mathrm{p}=0.118)$. The EP surface produced a $22 \%$ failure rate based on the LOI exclusion criteria ( 2 out of 9 ), with only $44 \%$ of the observations meeting the full biocompatibility benchmarks (4 out of 9 ). $100 \%$ of the AD samples ( 9 out of 9 ) met the full biocompatibility benchmarks, leading to a failure rate of $0 \%$ (0 out of 9$)$. To provide context with clinical observations of stent performance, bare metal stents incur a restenosis (failure) rate of between 20\%-30\%, with drug eluting stents reducing this value to $<10 \%$. Using the restenosis rate of present generation drug eluting stents $(\sim 10 \%)$ as a guideline, the AD surface is seen as potentially improving outcomes while the EP surface falls outside expectations for success. Although the AD coating employed here may not be appropriate for stents due to potential cracking of the layer during balloon crimping and deployment, this investigation highlights the critical importance of oxide films for determining the biocompatibility of degradable zinc metal implants.

\subsection{Conclusions}

An electropolished and anodized surface oxide film with widely varying characteristics were prepared on degradable zinc implants and compared, both in vitro and in vivo, to clarify the relationship between surface film properties and biocompatibility. The following conclusions were reached: 
The anodized samples exhibited a higher corrosion resistance compared to electropolished during longer exposure to the physiological solution due to the densification of the oxalate film, which acted as a barrier limiting the contact of the substrate with electrolyte.

The corrosion product film formed on electropolished samples was less compact, with varied thickness. Thus, it provided limited corrosion protection.

The electropolishing produced a smooth and thin oxide film surface that elicited a high variability in biocompatibility, with a high rate of failure, possibly due to surface corrosion heterogeneity.

In contrast, the corrosion stability of the anodized surface film corresponded to improved biocompatibility outcomes.

In particular, the ability to tune the in vivo biodegradation behavior and biocompatibility of the $\mathrm{Zn}$ biomaterial as a function of surface properties was experimentally verified

\subsection{Funding Sources}

U.S. National Institute of Health - National Institute of Biomedical Imaging and Bioengineering, Grant \#R21 EB 024034-01A1 and \#1R21EB019118-01A1

\subsection{ACKNOWLEDGMENT}

Research reported in this publication was supported by the U.S. National Institute of Health - National Institute of Biomedical Imaging and Bioengineering, Grant \#R21 EB 024034-01A1 and \#1R21EB019118-01A1. The content is solely the responsibility of the authors and does not necessarily represent the official views of the National Institutes of Health. Roger J. Guillory II was supported by the National Science Foundation Graduate Research Fellowship. 
The authors acknowledge Dr. Timothy Leftwich for his assistance with XPS, Mrs. Avishan Arab-Shomali for her assistance in the surface treatment procedures, and Dr. Ehsan Mostaed for assistance in SEM micrograph collection. The authors acknowledge the Applied Chemical and Morphological Analysis Laboratory at Michigan Tech for use of the instruments and staff assistance.

\subsection{References}

1. Bowen, P. K.; Shearier, E. R.; Zhao, S.; Guillory, R. J.; Zhao, F.; Goldman, J.; Drelich, J. W., Biodegradable Metals for Cardiovascular Stents: From Clinical Concerns to Recent Zn-Alloys. Advanced Healthcare Materials 2016, 5 (10), 1121-1140.

2. Seitz, J. M.; Durisin, M.; Goldman, J.; Drelich, J. W., Recent Advances in Biodegradable Metals for Medical Sutures: A Critical Review. Advanced Healthcare Materials 2015, 4 (13), 1915-1936.

3. Mostaed, E.; Sikora-Jasinska, M.; Drelich, J. W.; Vedani, M., Zinc-Based Alloys for Degradable Vascular Stent Applications. Acta Biomaterialia 2018, 71, 1-23.

4. Katarivas Levy, G.; Goldman, J.; Aghion, E., The Prospects of Zinc as a Structural Material for Biodegradable Implants-A Review Paper. Metals 2017, 7 (10), 402.

5. Hehrlein, C.; Schorch, B.; Kress, N.; Arab, A.; von zur Mühlen, C.; Bode, C.; Epting, T.; Haberstroh, J.; Mey, L.; Schwarzbach, H., Zn-Alloy Provides a Novel Platform for Mechanically Stable Bioresorbable Vascular Stents. PloS One 2019, 14 (1), e0209111. 6. Lin, S.; Ran, X.; Yan, X.; Yan, W.; Wang, Q.; Yin, T.; Zhou, J. G.; Hu, T.; Wang, G., Corrosion Behavior and Biocompatibility Evaluation of a Novel Zinc-Based Alloy Stent in Rabbit Carotid Artery Model. Journal of Biomedical Materials Research Part B: Applied Biomaterials 2018.

7. Yang, H.; Wang, C.; Liu, C.; Chen, H.; Wu, Y.; Han, J.; Jia, Z.; Lin, W.; Zhang, D.; Li, W., Evolution of the Degradation Mechanism of Pure Zinc Stent in the One-Year Study of Rabbit Abdominal Aorta Model. Biomaterials 2017, 145, 92-105.

8. Mostaed, E.; Sikora-Jasinska, M.; Mostaed, A.; Loffredo, S.; Demir, A.; Previtali, B.; Mantovani, D.; Beanland, R.; Vedani, M., Novel Zn-Based Alloys for Biodegradable Stent 
Applications: Design, Development and In Vitro Degradation. Journal of the Mechanical Behavior of Biomedical Materials 2016, 60, 581-602.

9. Sikora-Jasinska, M.; Mostaed, E.; Mostaed, A.; Beanland, R.; Mantovani, D.; Vedani, M., Fabrication, Mechanical Properties and In Vitro Degradation Behavior of Newly Developed ZnAg Alloys for Degradable Implant Applications. Materials Science and Engineering: C 2017, $77,1170-1181$.

10. Drelich, A. J.; Bowen, P. K.; LaLonde, L.; Goldman, J.; Drelich, J. W., Importance of Oxide Film in Endovascular Biodegradable Zinc Stents. Surface Innovations 2016, 4 (3), 133 140.

11. Drelich, A. J.; Zhao, S.; Guillory II, R. J.; Drelich, J. W.; Goldman, J., Long-Term Surveillance of Zinc Implant in Murine Artery: Surprisingly Steady Biocorrosion Rate. Acta Biomaterialia 2017, 58, 539-549.

12. Levy, G. K.; Kafri, A.; Ventura, Y.; Leon, A.; Vago, R.; Goldman, J.; Aghion, E., Surface Stabilization Treatment Enhances Initial Cell Viability and Adhesion for Biodegradable Zinc Alloys. Materials Letters 2019.

13. Shomali, A. A.; Guillory, R. J.; Seguin, D.; Goldman, J.; Drelich, J. W., Effect of PLLA Coating on Corrosion and Biocompatibility of Zinc in Vascular Environment. Surface Innovations 2017, 5 (4), 211-220.

14. Guillory, R. J.; Bowen, P. K.; Hopkins, S. P.; Shearier, E. R.; Earley, E. J.; Gillette, A. A.; Aghion, E.; Bocks, M.; Drelich, J. W.; Goldman, J., Corrosion Characteristics Dictate the Long-Term Inflammatory Profile of Degradable Zinc Arterial Implants. ACS Biomaterials Science \& Engineering 2016, 2 (12), 2355-2364.

15. Cipriano, A. F.; Lin, J.; Miller, C.; Lin, A.; Alcaraz, M. C. C.; Soria Jr, P.; Liu, H., Anodization of Magnesium for Biomedical Applications-Processing, Characterization, Degradation and Cytocompatibility. Acta Biomaterialia 2017, 62, 397-417.

16. Yuan, W.; Li, B.; Chen, D.; Zhu, D.; Han, Y.; Zheng, Y., Formation Mechanism, Corrosion Behavior, and Cytocompatibility of Microarc Oxidation Coating on Absorbable HighPurity Zinc. ACS Biomaterials Science \& Engineering 2018.

17. Yun, Y.; Dong, Z.; Lee, N.; Liu, Y.; Xue, D.; Guo, X.; Kuhlmann, J.; Doepke, A.; Halsall, H. B.; Heineman, W., Revolutionizing Biodegradable Metals. Materials Today 2009, 12 (10), 22-32. 
18. Asri, R.; Harun, W.; Samykano, M.; Lah, N.; Ghani, S.; Tarlochan, F.; Raza, M., Corrosion and Surface Modification on Biocompatible Metals: A Review. Materials Science Engineering: $C$

2017, 77, 1261-1274.

19. Mousa, H. M.; Park, C. H.; Kim, C. S., Surface Modification of Magnesium and its Alloys Using Anodization for Orthopedic Implant Application. In Magnesium Alloys, InTech: 2017.

20. Diamanti, M.; Pedeferri, M., The Anodic Oxidation of Titanium and Its Alloys. In Encyclopedia of Interfacial Chemistry: Surface Science and Electrochemistry, 2018; pp 41-54.

21. Gellér, Z. E.; Albrecht, K.; Dobránszky, J. In Electropolishing of Coronary Stents, Materials Science Forum, Trans Tech Publ: 2008; pp 367-372.

22. Cobley, A., Electropolishing: Electrolytic Brightening, Smoothing and Deburring of Stainless Steel, Brass, Copper, Aluminium, Titanium and Magnesium. Taylor \& Francis: 2017; Vol. 95, p 1.

23. Tepe, G.; Wendel, H. P.; Khorchidi, S.; Schmehl, J.; Wiskirchen, J.; Pusich, B.; Claussen, C. D.; Duda, S. H., Thrombogenicity of Various Endovascular Stent Types: An In Vitro Evaluation. Journal of Vascular and Interventional Radiology 2002, 13 (10), 1029-1035. 24. Kaufmann, C.; Mani, G.; Marton, D.; Johnson, D.; Agrawal, C. M., Long-Term Stability of Self-Assembled Monolayers on Electropolished L605 Cobalt Chromium Alloy for Stent Applications. Journal of Biomedical Materials Research Part B: Applied Biomaterials 2011, 98 (2), 280-289.

25. Chen, C.-Y.; Ozasa, K.; Katsumata, K.-i.; Maeda, M.; Okada, K.; Matsushita, N., Bioactive Titanium Oxide-Based Nanostructures Prepared by One-Step Hydrothermal Anodization. The Journal of Physical Chemistry C 2012, 116 (14), 8054-8062.

26. Zhou, R.; Wei, D.; Cao, J.; Feng, W.; Cheng, S.; Du, Q.; Li, B.; Wang, Y.; Jia, D.; Zhou, Y., Synergistic Effects of Surface Chemistry and Topologic Structure From Modified Microarc Oxidation Coatings on Ti Implants for Improving Osseointegration. ACS Applied Materials \& Interfaces 2015, 7 (16), 8932-8941.

27. ASTM, Standard Guide for Electrolytic Polishing of Metallographic Specimens. In E1558-09, West Conshohocken,PA, 2014. 
28. Sanz, O.; Echave, F. J.; Odriozola, J. A.; Montes, M., Aluminum Anodization in Oxalic Acid: Controlling the Texture of A12O3/Al Monoliths for Catalytic Applications. Industrial \& Engineering Chemistry Research 2011, 50 (4), 2117-2125.

29. Feliu Jr, S.; Barranco, V., XPS Study of the Surface Chemistry of Conventional Hot-Dip Galvanised Pure Zn, Galvanneal and Zn-Al Alloy Coatings on Steel. Acta Materialia 2003, 51 (18), 5413-5424.

30. Mutel, B.; Taleb, A. B.; Dessaux, O.; Goudmand, P.; Gengembre, L.; Grimblot, J., Characterization of Mixed Zinc-Oxidized Zinc Thin Films Deposited by a Cold Remote Nitrogen Plasma. Thin Solid Films 1995, 266 (2), 119-128.

31. Ferreira Jr, J.; Souza, K.; Queiroz, F.; Costa, I.; Tomachuk, C., Electrochemical and Chemical Characterization of Electrodeposited Zinc Surface Exposed to New Surface Treatments. Surface Coatings Technology 2016, 294, 36-46.

32. Deroubaix, G.; Marcus, P., X-Ray Photoelectron Spectroscopy Analysis of Copper and Zinc Oxides and Sulphides. Surface Interface Analysis 1992, 18 (1), 39-46.

33. Guillory, R. J.; Oliver, A. A.; Davis, E. K.; Earley, E. J.; Drelich, J. W.; Goldman, J., Preclinical In Vivo Evaluation and Screening of Zinc-Based Degradable Metals for Endovascular Stents. Journal of Metals 2019, 1-11.

34. Sinha, M. K.; Pramanik, S.; Sahu, S. K.; Prasad, L. B.; Jha, M. K.; Pandey, B. D., Development of An Efficient Process for the Recovery of Zinc and Iron as Value Added Products From the Waste Chloride Solution. Sep Purif Technol 2016, 167, 37-44.

35. Ramirez-Canon, A.; Miles, D. O.; Cameron, P. J.; Mattia, D., Zinc Oxide Nanostructured Films Produced Via Anodization: A Rational Design Approach. Rsc $A d v$ 2013, 3 (47), 25323-25330.

36. Miller, D.; Biesinger, M.; McIntyre, N., Interactions of CO2 and CO at Fractional Atmosphere Pressures With Iron and Iron Oxide Surfaces: One Possible Mechanism for Surface Contamination? Surface Interface Analysis 2002, 33 (4), 299-305.

37. Barr, T. L.; Seal, S., Nature of the use of Adventitious Carbon as a Binding Energy Standard. Journal of Vacuum Science Technology A: Vacuum, Surfaces, Films 1995, 13 (3), 1239-1246.

38. Bai, X.; Tran, T. H.; Yu, D.; Vimalanandan, A.; Hu, X.; Rohwerder, M., Novel Conducting Polymer Based Composite Coatings for Corrosion Protection of Zinc. Corrosion Science 2015, 95, 110-116. 
39. Aramaki, K., Effects of Organic Inhibitors on Corrosion of Zinc in an Aerated 0.5 M $\mathrm{NaCl}$ Solution. Corrosion Science 2001, 43 (10), 1985-2000.

40. Lebrini, M.; Fontaine, G.; Gengembre, L.; Traisnel, M.; Lerasle, O.; Genet, N., Corrosion Protection of Galvanized Steel and Electroplating Steel by Decanoïc Acid in Aqueous Solution: Electrochemical Impedance Spectroscopy, XPS and ATR-FTIR. Corrosion Science 2009, 51 (6), 1201-1206.

41. Bowen, P. K.; Drelich, J.; Goldman, J., Zinc Exhibits Ideal Physiological Corrosion Behavior For Bioabsorbable Stents. Advanced Materials 2013, 25 (18), 2577-2582.

42. Liu, X.; Yang, H.; Liu, Y.; Xiong, P.; Guo, H.; Huang, H.-H.; Zheng, Y., Comparative Studies on Degradation Behavior of Pure Zinc in Various Simulated Body Fluids. Journal of Metals 2018, 71 (4), 1414-1425.

43. Champagne, S.; Mostaed, E.; Safizadeh, F.; Ghali, E.; Vedani, M.; Hermawan, H., In Vitro Degradation of Absorbable Zinc Alloys in Artificial Urine. Materials 2019, 12 (2), 295.

44. Törne, K.; Larsson, M.; Norlin, A.; Weissenrieder, J., Degradation of Zinc in Saline Solutions, Plasma, and Whole Blood. Journal of Biomedical Materials Research Part B: Applied Biomaterials 2016, 104 (6), 1141-1151.

45. Törne, K. B.; Örnberg, A.; Weissenrieder, J., Characterization of the protective layer formed on zinc in whole blood. Electrochimica Acta 2017, 258, 1476-1483.

46. Zhang, X. G., Corrosion and Electrochemistry of Zinc. Springer Science \& Business Media: 2013.

47. Compere, C.; Frechette, E.; Ghali, E., The Corrosion Evaluation of Painted and Artificially Damaged Painted Steel Panels by Ac-Impedance Measurements. Corrosion Science 1993, 34 (8), 1259-1274.

48. Bowen, P. K.; Guillory II, R. J.; Shearier, E. R.; Seitz, J.-M.; Drelich, J.; Bocks, M.; Zhao, F.; Goldman, J., Metallic Zinc Exhibits Optimal Biocompatibility for Bioabsorbable Endovascular Stents. Materials Science and Engineering: C 2015, 56, 467-472.

49. Lu, J.; Rao, M. P.; MacDonald, N. C.; Khang, D.; Webster, T. J., Improved Endothelial Cell Adhesion and Proliferation on Patterned Titanium Surfaces With Rationally Designed, Micrometer to Nanometer Features. Acta Biomaterialia 2008, 4 (1), 192-201.

50. Potthoff, E.; Franco, D.; D’Alessandro, V.; Starck, C.; Falk, V.; Zambelli, T.; Vorholt, J. A.; Poulikakos, D.; Ferrari, A., Toward a Rational Design of Surface Textures Promoting Endothelialization. Nano Letters 2014, 14 (2), 1069-1079. 


\section{In-Vivo Biocompatibility of Next Generation Zn-Ag Based Stent Materials ${ }^{7}$}

\subsection{Introduction}

Since the introduction of zinc as a degradable metallic candidate for bioresorbable stents in 2013[1], numerous zinc alloy formulations with improved mechanical properties and microstructure relative to pure zinc have been investigated [2-5]. A central feature of the zinc-based stent materials being developed is their gradual degradation in the arterial environment, until their eventual disappearance $[6,7]$. The biodegradable feature of zincbased materials makes them highly interactive with the host environment. Although bioresorbable implants differ considerably from conventional biostable implants in terms of their interaction with the host, most studies provide limited details regarding the biological effects evoked by the implant's presence and degradation. Consequently, there remains a wide deficit in our understanding of the impact of alloying elements and bulk processing on the tissue response to zinc-based implants. The biointeractivity of zincbased materials needs to be thoroughly characterized in order to engineer zinc-based implants with improved safety and efficacy relative to their bioinert metallic counterparts.

Our group has worked to clarify the relationship between the material composition, properties and surface finish of degrading zinc implants and the biologic responses they evoke in the arterial environment [8-15]. We make use of an implant model wherein a wire made of the material in question is implanted into the abdominal aorta of healthy Sprague Dawley rats and allowed to degrade for 3-20 months[9, 12, 13]. Recently, we developed a histomorphometric approach to quantify the performance of bioresorbable materials in the arterial environment [11]. This work has identified thresholds that distinguish between acceptable materials and those that stimulate a harmful smooth muscle cell proliferative response, a hallmark failure of long-term implanted stents.

\footnotetext{
${ }^{7}$ Material in this chapter is in preparation to be submitted to a journal for publication
} 
Of all the zinc-based implants evaluated so far, pure zinc generally evokes the most favorable response within the vascular environment. A stable neointima forms around pure zinc that is restricted in terms of area, thickness, and progression and generally avoids inflammatory infiltrates for up to 11 months. We recently reported that alloying with magnesium contributes to the biological failure of zinc implants through excessive tissue growth [11]. Although the implantation of a $\mathrm{Zn}-4 \mathrm{Li}$ alloys into the arterial environment did not produce outright failures, this alloy experienced a reduced biocompatibility metric relative to pure zinc due to an increased degree of inflammation. In addition to modifications to the bulk matrix, we have also shown that the initial surface oxide film characteristics dramatically affect the early neointimal growth [9][16]. For instance, thin surface oxide films produced by conventional electropolishing are susceptible to large variations in corrosive attack that lead to excessive variations in biological responses[9]. In contrast, relative stability of the surface oxide film (imparted for example through anodization) promotes a more homogenous corrosion along the length of the wire, which results in more positive and reproducible biological responses. In contrast to some success seen in modifications of the surface[9, 16], manipulating the bulk metal through alloying to improve the mechanical properties has tended to reduce the overall biocompatibility of the implant material relative to pure zinc.

As metallurgical modifications continue to advance and alloy compositions become more complex, it has become more urgent to clarify the relationship between material characteristics and tissue response. Such clarifications may provide predictive power for metallurgists to aid in the design of zinc-based medical implants. More recent zinc systems include 2-4 alloying elements to refine the grain structure, increase microstructural stability, and improve the mechanical properties of zinc to acceptable levels for stenting applications [2, 4]. The addition of each element impacts corrosion behavior and products, secondary phases within the microstructure, and surface characteristic, all of which can affect the overall device biocompatibility at various stages after implantation. A deeper understanding of the relationships between material modifications and biological responses is needed to identify desirable material 
characteristics from a biocompatibility standpoint, providing a foundation for metallurgical engineering of bioresorbable medical alloys.

Recently, a new $\mathrm{Zn}$ alloy system has been introduced with the addition of $\mathrm{Ag}[17]$. This system benefits from an increased volume fraction of $\mathrm{AgZn}_{3}$ particles that precipitate at the grain boundary and provide grain boundary strengthening due to impaired propagation of dislocation slip plains, as well as increased grain refinement (grain size $1.5 \mu \mathrm{m})[18]$. The Ag addition dramatically increases the ultimate tensile strength to 280$290 \mathrm{MPa}$ without sacrificing elongation to failure, and provides a beneficial antibacterial effect $[2,18]$.

Here we leverage high sample sizes to investigate the biocompatibility of 3 novel $\mathrm{Zn}$ based Ag-containing alloys, ranging from binary to quinary alloy systems. This work was intended to clarify the relationship between elemental profile and biocompatibility for the $\mathrm{Zn}-\mathrm{Ag}$ system. Selected binary and quinary Zn-Ag-based alloys underwent thermal treatment (TT) to increase the solubility of Ag-rich phases within the $\mathrm{Zn}$ bulk matrix, yielding 2 different microstructures with the same elemental composition. A side-by-side comparison of implant materials with and without TT was intended to clarify the effect of microstructural features on biocompatibility. The insight gained from these experiments is needed in order to integrate biological considerations into the metallurgical design of fine/ultrafine-grained structure Zn-based materials through alloying and processing.

\subsection{Methods}

\subsubsection{Alloy Formulation}

High-purity starting metals $(>99.99 \%)$ were melted at $700^{\circ} \mathrm{C}$ in a sealed cylindrical graphite crucible in an electric resistance furnace to minimize oxidation and ensure that sound castings are produced. The ingots had a cylindrical shape with a nominal diameter of $28 \mathrm{~mm}$ and a length of $100 \mathrm{~mm}$. The bars were machined to a nominal diameter of 25 $\mathrm{mm}$ and homogenized for 8 hours at approximately $400^{\circ} \mathrm{C}$ followed by water quenching. 
Nominal elemental formulations were used as outlined below:

$$
\begin{array}{ll}
\mathrm{Zn}-4 \mathrm{Ag}(\mathrm{wt} \%) & \text { (binary) } \\
\mathrm{Zn}-4 \mathrm{Ag}-0.6 \mathrm{Mn}(\mathrm{wt} \%) & \text { (ternary) } \\
\mathrm{Zn}-4 \mathrm{Ag}-0.8 \mathrm{Cu}-0.6 \mathrm{Mn}-0.15 \mathrm{Zr}(\mathrm{wt} \%) & \text { (quinary) }
\end{array}
$$

The homogenized alloys were subsequently extruded at $310^{\circ} \mathrm{C}$ with an extrusion rate and speed of 39:1 and $0.5 \mathrm{~mm} / \mathrm{min}$, respectively, to obtain cylindrical rods with a diameter of $4 \mathrm{~mm}$. The extruded rods were cold drawn at Fort Wayne Metals (Fort Wayne, IN) to a wire with a diameter of $0.25 \mathrm{~mm}$.

The binary and quinary alloys were heat treated in order to minimize the Ag-rich precipitates. For the binary alloy, TT was performed at $360^{\circ} \mathrm{C}$ for $10 \mathrm{~s}$, while the quinary alloy, due to its multiplicity of alloying elements, required higher temperature and soaking time $\left(390^{\circ} \mathrm{C}\right.$ for $\left.20 \mathrm{~min}\right)$.

\subsubsection{TEM preparation}

Transmission electron microscopy (TEM) samples were prepared using standard routes, including grinding and polishing the wires to $<40 \mathrm{~mm}$ in thickness followed by ion beam milling to electron transparency. Low angle-ion beam milling was performed at liquid nitrogen temperature by a GATAN precision ion polishing (PIPS) II using Ar ${ }^{+}$initially at $5 \mathrm{keV}$ and final milling at $1 \mathrm{keV}$. TEM data were obtained with JEOL JEM F200 transmission electron microscopy operating at $200 \mathrm{kV}$.

\subsubsection{Implantation and collection}

Implantations, implant retrieval, and histological processing were performed as previously described [11]. Wires were implanted into female Sprague Dawley rats for 6 months. Sixteen samples were produced for each material. 


\subsubsection{Statistical analysis}

All data was tabulated in Excel 2016 and uploaded to MATLAB 2016Ra. Raw data was transformed into log space for statistical comparisons. An unpaired two sample Welch's $t$ test was used to investigate the experimental groups. When appropriate, an F test was used to identify differences in the variance. All data was approximately normal.

\subsection{Results and Discussion:}

\subsubsection{Microstructural Characterization}
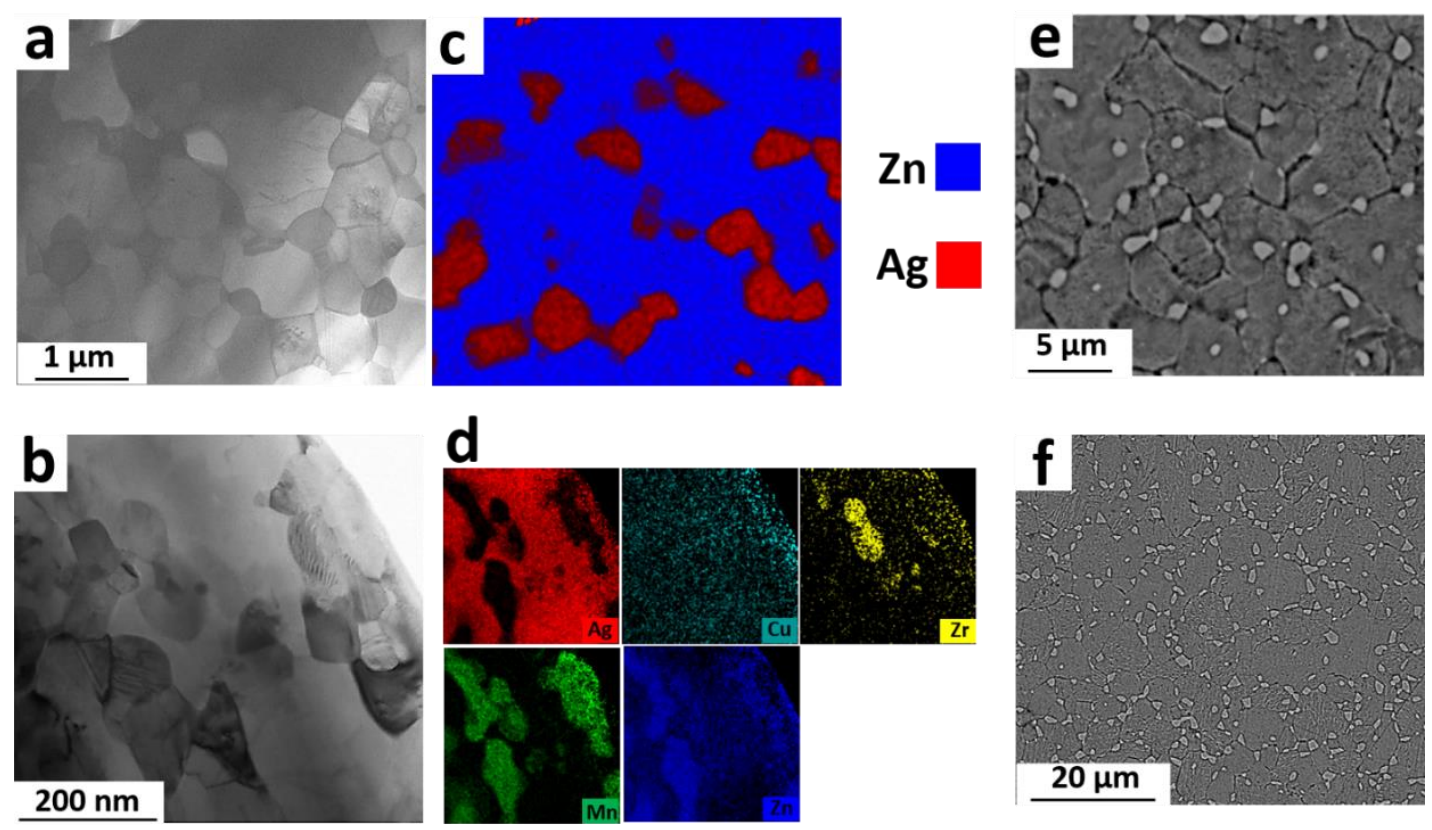

Fig. 4-1. (a, b) TEM images of as-drawn binary and quinary alloys, (c, d) their corresponding EDS elemental 
Fig. 4-1 shows the transmission electron microscopy (TEM) images of the binary and quinary alloys before and after the thermal treatments. It can be clearly seen form Fig. 4$1 \mathrm{a}$ and $\mathrm{b}$ that for both alloys the wire drawing results in the formation of an ultrafinegrained (UFG) structure (grain size of $600 \mathrm{~nm}$ and 200nm for binary and ternary alloys, respectively). Formulated alloys underwent tensile testing and microstructure analysis. However, because mechanical properties of alloys are irrelevant to biocompatibility study they will be presented in a separate publication. Only selected mechanical data are provided in this section to guide general correlations between microstructure and mechanical properties of the alloys.

Fig. 4-1 shows the transmission electron microscopy (TEM) images of the binary and quinary alloys before and after the thermal treatments. For both alloys the wire drawing resulted in the formation of an ultrafine-grained (UFG) structure with grain size of $600 \mathrm{~nm}$ and $200 \mathrm{~nm}$ for binary and ternary alloys, respectively (Fig. 4-1a\&b). An EDS elemental map of the as-drawn binary alloy wire demonstrates the formation of submicron deformation induced- $\mathrm{AgZn}_{3}$ precipitates within a size range of $100 \mathrm{~nm}-500 \mathrm{~nm}$ (red particles) uniformly dispersed in the $\mathrm{Zn}$ matrix (Fig. 4-1c). The formation of these precipitates markedly enhances the ductility such that the material exhibits exceptional room temperature superplasticity (fracture elongation $>400 \%$ ). However, this superplasticity is accompanied by a remarkable loss of mechanical strength $(\sim 73 \%)$. In the case of the quinary alloy (Fig. 4-1b and d) the considerably smaller grain size relative to the binary alloy (200 nm vs $600 \mathrm{~nm}$ ) is attributed to the extremely refined and uniformly dispersed MnZr-rich particles. Such particles hinder the growth of the newly recrystallized UFG grains during the drawing process, resulting in markedly smaller grain size. The EDS elemental map of the quinary alloy shows that the addition of $\mathrm{Cu}$ appreciably decreases the fraction of $\mathrm{AgZn}_{3}$ precipitates (Fig. 4-1d). This results in a lower fracture elongation of the quinary relative to the binary alloy (90\% vs $430 \%)$. To restore the mechanical properties, thermal treatments were carried out on the as-drawn alloys. Fig. 4-1e shows that for the binary alloy, $\mathrm{TT}$ at $360^{\circ} \mathrm{C}$ for $10 \mathrm{~s}$ leads to dissolution of a large number of the $\mathrm{AgZn}_{3}$ precipitates as well as a significant grain growth. In fact, the remarkable decrease in the 
volume fraction of $\mathrm{AgZn}_{3}$ precipitates in TT binary alloy could restore almost $73 \%$ of the mechanical strength. In the case of the quinary alloy, TT was conducted at a significantly higher temperature and for a longer soaking time $\left(390^{\circ} \mathrm{C}\right.$, which corresponds to $96 \%$ of the alloy's melting point, for $20 \mathrm{~min}$ ). Fig. 4-1f reveals that, surprisingly, despite the very high temperature, only a small grain growth occurs (from $200 \mathrm{~nm}$ to $2.4 \mu \mathrm{m}$ ). This is attributed to the greatly fine and uniformly distributed MnZr-rich intermetallic particles at grain boundaries, which are outstandingly thermally stable, that prevent grain coarsening even at high temperatures. Indeed, the TT quinary alloy could substantially restore its mechanical strength (around 84\%) while exhibiting a high fracture elongation of $32 \%$.

\subsubsection{General Histologic Presentation}
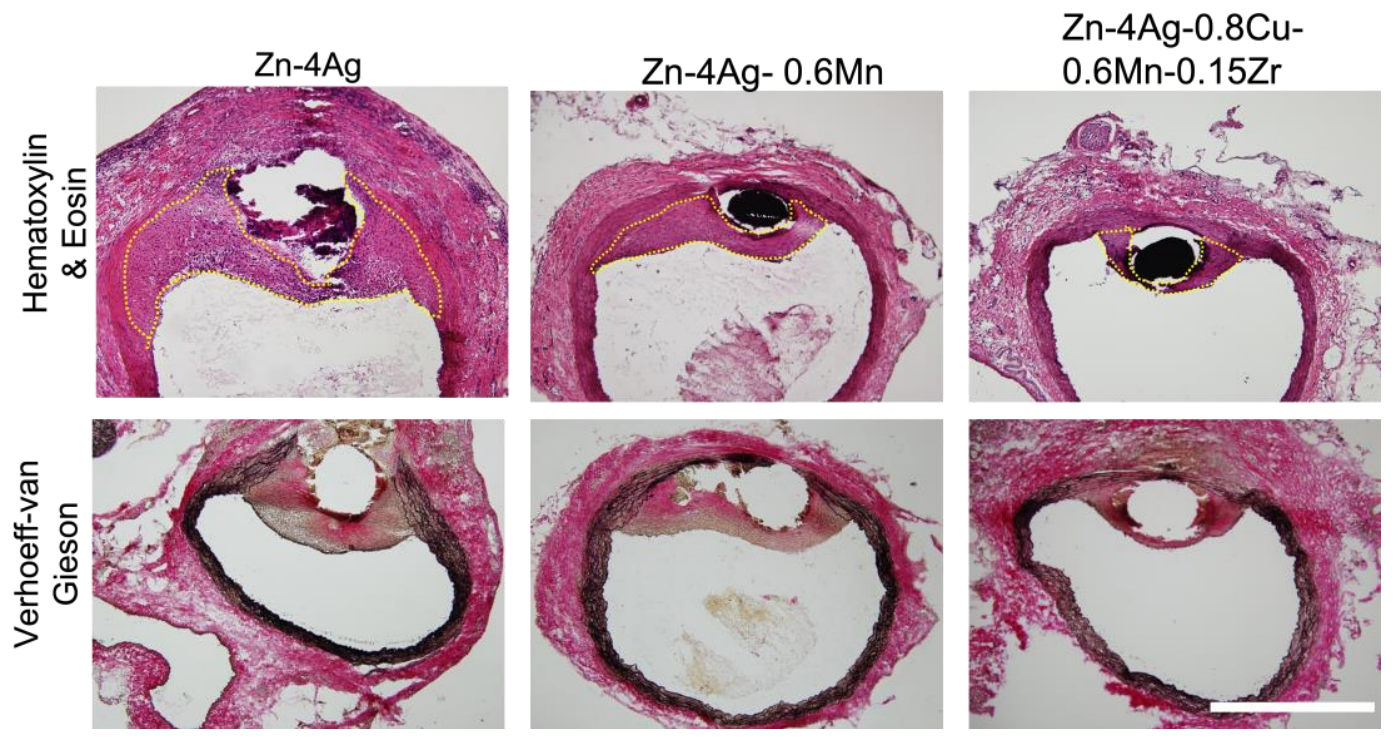

Fig.4-2. Representative H\&E and VVG stains of the binary, ternary, and quinary alloys. The neointima is outlined in yellow to increase clarity. Scale bar is approximately $500 \mu \mathrm{m}$

The general histological presentation of the tested alloys is shown in Fig. 4-2. Typical binary $\mathrm{Zn}-4 \mathrm{Ag}$, ternary $\mathrm{Zn}-4 \mathrm{Ag}-0.6 \mathrm{Mn}$, and quinary $\mathrm{Zn}-4 \mathrm{Ag}-0.8 \mathrm{Cu}-0.6 \mathrm{Mn}-0.15 \mathrm{Zr}$ implants are shown with $\mathrm{H} \& \mathrm{E}$ and VVG stains. H\&E staining is ideal for microscopic cell identification and characterization of the general tissue reaction, while the VVG technique clearly differentiates elastic lamina fibers and neointimal tissue. Since we are 
mainly concerned with the macro neointimal development around each implanted alloy, the VVG stain is primarily used for ease of histomorphometric measurements.

The binary and ternary alloys display excessive neointmal thickness and area, with the quinary alloy showing a reduced, ideal neointimal type at 6 months. The binary and ternary alloys show evidence of increased inflammation within the neointima, by H\&E identification of inflammatory cells (not shown). This was largely absent for the quinary alloy, with a reduced cell density and stable neointima growth that does not appear to be progressive. 


\subsubsection{Effects of alloying}
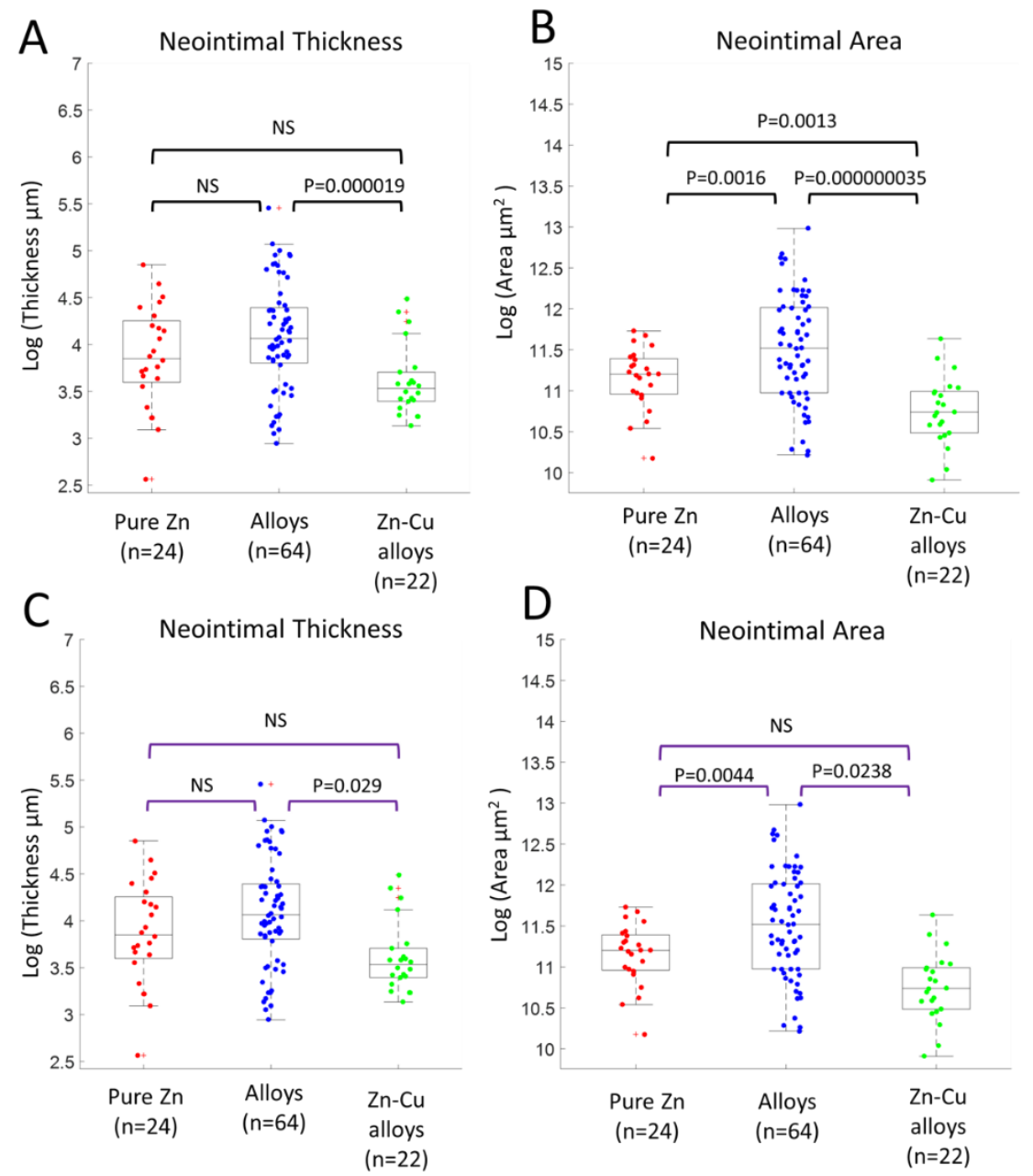

Fig.4-3. Boxplots of Pure $\mathrm{Zn}$, copper-free alloys, and Cu containing alloys for histomorphometrics. Panels A and B show comparisons using a Welch's two sample t test. Panels $C$ and $D$ show comparisons of the variance with a two sample $F$ test.

The effects of alloying and processing on the in vivo performance of degradable zinc materials was compared in terms of histomorphometry parameters, using an approach previously described [11]. Fig. 4-3 shows a high sample size pooled performance of alloys implanted from 3-12 months, including pure zinc, multiple copper-free alloy 
systems (Zn-4Ag, Zn-4Ag-0.6Mn, Zn-4Li, and Zn-XMg [0.1,0.001,0.001wt\%]), and the $\mathrm{Zn}-4 \mathrm{Ag}-0.8 \mathrm{Cu}-0.6 \mathrm{Mn}-0.15 \mathrm{Zr}$ alloy developed by our group. In vivo performance was quantified by neointimal thickness (WLT) and neointmal area (NA). Interestingly, a similar WLT was found between the copper-free alloys and the quinary system relative to pure zinc (Fig. 4-3A). However, there is a clear superior performance of the coppercontaining quinary alloys relative to the copper-free alloys, $(\mathrm{P}=0.000019)$.

The copper-free alloys generally incur a reduced biocompatibility relative to pure $\mathrm{Zn}$ in terms of NA, with a higher sample mean $(\mathrm{P}=0.0016)$. The NA for the copper-containing alloy is significantly reduced relative to the copper-free alloys ( $\mathrm{P}=0.0000000035)$, and even improved over that of pure zinc. We attribute the reduction in NA to a reduced inflammation at the interface of the $\mathrm{Cu}$ containing alloy (Fig. 4-2).

A previously described lumen occlusion index (LOI) was used to compare novel alloys in terms of negative proliferative responses that occludes the lumen. Surprisingly, none of the new alloys exceeded the biocompatibility limit LOI value of $\geq 30 \%$, or the reduction in compatibility limit LOI of or $\geq 20 \%$. The excellent LOI values for the $\mathrm{Zn}-\mathrm{Ag}$ alloys may be due to their extended base neointimal length (BNL), along with typical WLT values (which could indicate positive remodeling). Although none of the implants failed outright, the large NA produced by the binary and ternary alloys is concerning. This large increase in area is primarily due to an increase in inflammation, which is a well-known contributor to negative stent outcomes. The LOI measurement does not take into account NA, but rather relates to the protrusive growth of the neointima into the luminal space. Our lab has found that a neointimal protrusion metric is generally related to smooth muscle cell proliferation, while a large increase in NA is generally indicative of excessive inflammation[11]. Both factors should be considered when gauging the biocompatibility of degradable materials, especially since satisfactory performance on the LOI scale does not demonstrate optimal performance in all histological metrics.

Sample variance between the experimental materials is a major consideration when evaluating histological samples. Tighter data spread is associated with more uniform 
performance. This feature is especially valuable in the intra luminal environment, which is subject to occlusion at any point along the length of the implant [9]. Pure zinc generally exhibits an ideal histological variance, avoiding corrosion behavior that provokes large changes in neointimal growth along the length of the implant. While there are generally no differences in the variance of WLT between pure zinc implants and the copper-free alloys, the copper-containing quinary alloys demonstrate a much tighter data spread than copper-free alloys $(\mathrm{P}=0.029$, Fig.4-3c $)$. NA measurements for the copper-free alloys are generally more variable relative to both pure $\mathrm{Zn}(\mathrm{P}=0.0044)$ and coppercontaining alloys $(\mathrm{P}=0.0238)$. The quinary alloy and pure $\mathrm{Zn}$ exhibit a similar variance, suggesting a more ideal performance from copper-containing alloys in terms of generating consistent neointimal morphomety.

Elemental composition is a serious consideration when designing alloys. When the binary $\mathrm{Zn}$-Ag composition was alloyed with Mn, the biocompatibility (BC) was considerably reduced. The $\mathrm{BC}$ then recovered following the addition of $\mathrm{Zr}$ and $\mathrm{Cu}$. While it can be difficult to isolate the effects of the Mn addition (which can alter corrosion behavior and form new inter metallics), it is apparent from our results that changes in the elemental profile of a degradable zinc implant can dramatically impact the BC. 


\subsubsection{Effects of nano-precipitate phases}

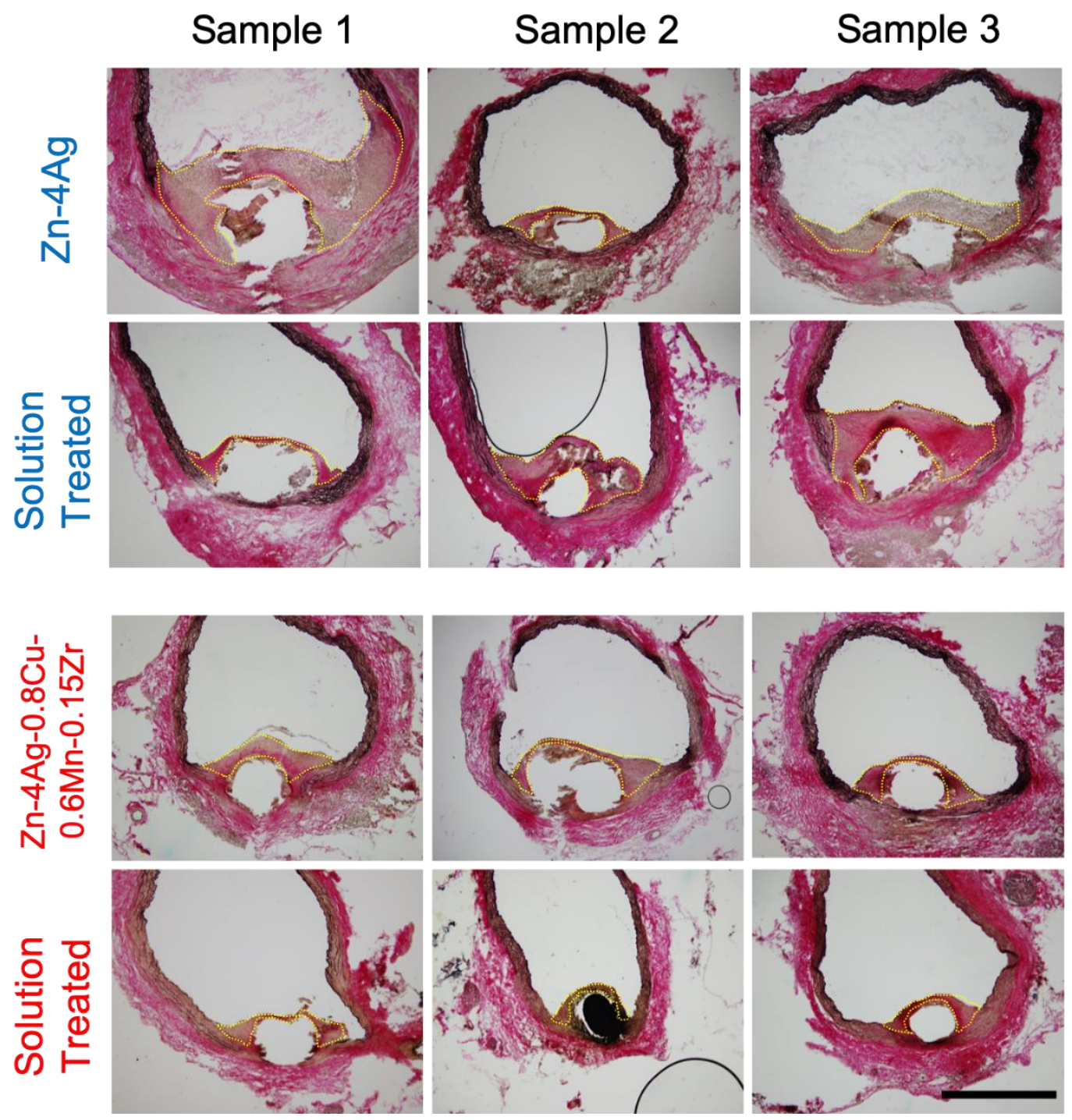

Fig.4-4 Three representative VVG stained sections of the binary and quinary alloys. High variability in neointimal size can be seen in both the binary and its solution treated counterpart. A decrease in overall NA is seen in the quinary alloy, with an even greater reduction in its solution treated counterpart. Scale bar is approximately $500 \mu \mathrm{m}$. 
In order to clarify the effects of nano-precipitate phases on neointimal growth, the binary and quinary $\mathrm{Zn}-\mathrm{Ag}$ alloys were thermally processed to dissolve the insoluble deformation induced-AgZn 3 nano-precipates. Applying TT has the benefit of reducing the amount of precipitates and thereby restoring the mechanical strength of the alloy [19].

Fig.4-4 shows the in vivo performance of both as drawn and solution treated binary and quinary alloys. For each alloy, three representative samples are depicted to demonstrate typical responses of the alloy system. For the binary system, large sample-to-sample differences are present in the NA (outlined in each image). The variability between samples does not appear to change after solution treatment (Fig.4-4 second row). The quinary system shows a marked reduction in NA relative to the binary system, along with a decreased variance. Solution treatment of the quinary alloy further reduces the NA and WLT, demonstrating the benefit of the solution treatment to biocompatibility.

The complete histomorphometric analysis of these samples is shown in Fig. 4-5. As shown in Figs 4-5A and 4B, WLT (but not NA) slightly benefitted from the solution treatment of the Zn-Ag binary alloy. The Zn-Ag and Zn-Ag TT materials both evoked an increased inflammation relative to pure zinc, which we attribute to the presence of $\mathrm{Ag}$ precipitates. It is well known that Ag nanoparticles contribute to inflammatory phenotypes in macrophages and elicit direct cell toxicity [20,21]. Therefore, a more pronounced reduction in Ag precipitates (obtainable by increased treatment time or temperature) may be beneficial in terms of biocompatibility.

The quinary alloy containing $\mathrm{Ag}$ and $\mathrm{Cu}$ possesses the same $\mathrm{Ag}$ nano precipates. Interestingly, after thermal treatment of the $\mathrm{Zn}-\mathrm{Cu}$ containing alloy, in vivo performance was dramatically improved, and even superior to pure zinc. WLT $(\mathrm{P}=0.023)$ and NA $(\mathrm{P}=0.000028)$ from the $\mathrm{Zn}-\mathrm{Cu}-\mathrm{TT}$ alloy readily outperforms pure zinc. It is interesting that the solution treatment for the binary alloy did not improve the $\mathrm{BC}$ as dramatically as for the quinary system. The solution treated binary alloy microstructure retains 
substantial Ag precipitates, possibly a consequence of the low ST time that was chosen in order to optimize the mechanical properties. Although the untreated quinary alloy also possesses Ag nano-precipitates, we speculate that the beneficial cellular effects from the addition of $\mathrm{Cu}$ overwhelms the negative response that is generated from these harmful phases.

Fig.4-5. Comparisons of the histomorphometric performance of binary $\mathrm{Zn}-\mathrm{Ag}$

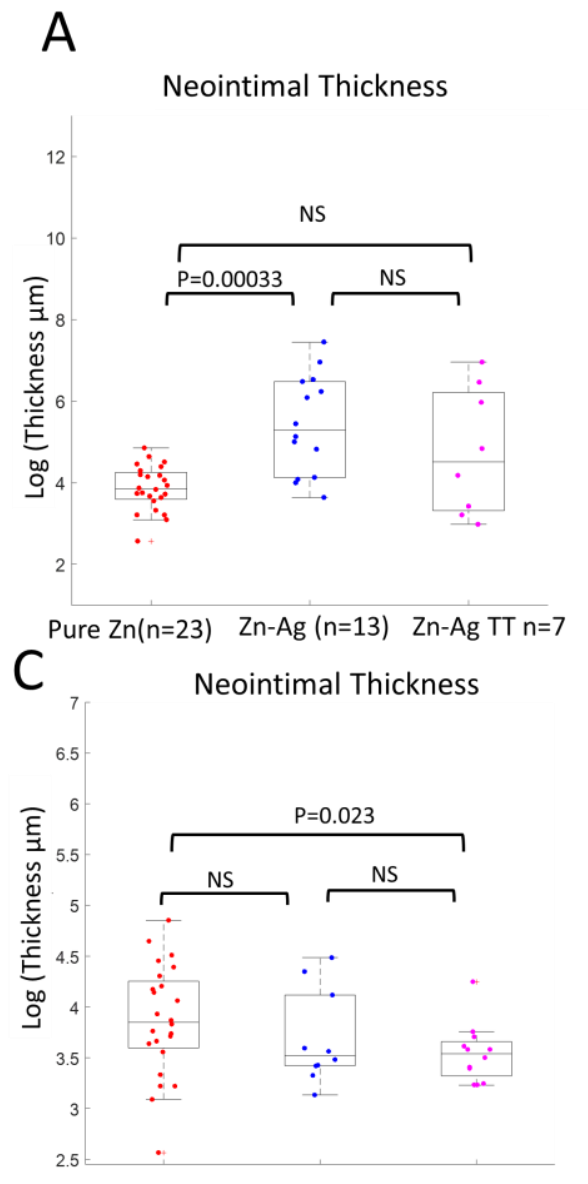

Pure Zn(n=23) Zn-Cu (n=7) Zn-Cu TT $n=11$

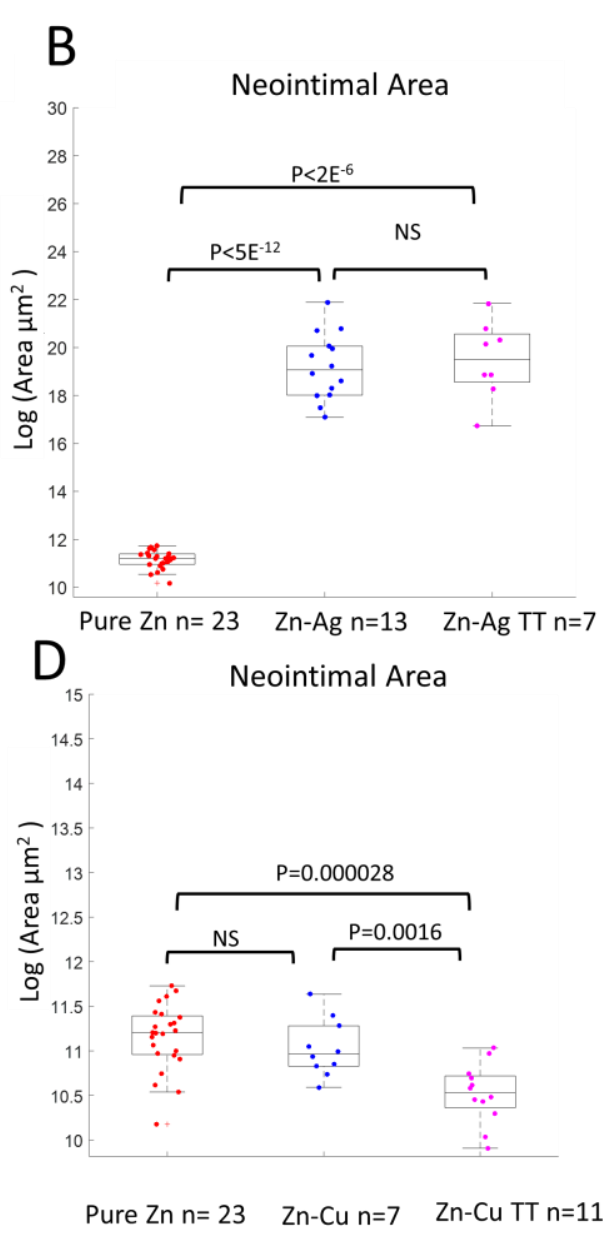

and quinary $\mathrm{Zn}-\mathrm{Ag}-\mathrm{Mn}-\mathrm{Zr}-\mathrm{Cu}$ alloys in the as-drawn form and after solution heat treatment. All comparisons were made with a two sample Welch's t test. 
We are the first to report a zinc-based alloy $(\mathrm{Zn}-4 \mathrm{Ag}-0.8 \mathrm{Cu}-0.6 \mathrm{Mn}-0.15 \mathrm{Zr}(\mathrm{wt} \%))$ that performs better than pure zinc in terms of biocompatibility. We speculate that the $\mathrm{Cu}$ inclusion has a beneficial impact on inflammatory development and neointimal growth. $\mathrm{Cu}$ has been shown to increase the proliferation of endothelial cells, but not smooth muscle cells [22]. In a proinflamatory environment such as a newly injured artery, increased scavenging of reactive oxygen species could have a beneficial effect on neointimal progression and inflammation. $\mathrm{Cu}$ can also directly act as a catalyst for the generation of NO from endogenous RSNO's, which could also inhibit inflammation [23] and SMC growth [24]. Furthermore, increased superoxide dismutase (SOD) activity in EC's could promote a favorable balance towards additional NO release [25].

\subsection{Conclusions}

The findings of the study conclude:

- Addition of alloying elements to zinc usually decreases the biocompatibility of zinc implant in vascular environment by increasing inflammation responses;

- Ag-rich nano-precipatites decrease biocompatibility, a phenomenon that can be counteracted by dissolving the $\mathrm{AgZn}_{3}$ precipates in the bulk $\mathrm{Zn}$ matrix;

- $\mathrm{Cu}$ addition to $\mathrm{Zn}-\mathrm{Ag}$ alloy reduces inflammation and substantially increases biocompatibility, even in the prescence of harmful $\mathrm{AgZn}_{3}$ precipitates, an effect that is enhanced when the precipitates are dissolved in the $\mathrm{Zn}$ matrix; and

- Neointimal forming cells are responsive to elemental additions and microstructural changes in degradable zinc based materials.

\subsection{Acknowledgments}

Research reported in this publication was supported by the U.S. National Institute of Health - National Institute of Biomedical Imaging and Bioengineering, grant \#R21 EB 024034-01A1 and \#1R21EB019118-01A1. The content is solely the responsibility of the authors and does not necessarily represent the official views of the National Institutes of 
Health. R.J.G. was supported by the National Science Foundation Graduate Research Fellowship.

\subsection{References}

[1] P.K. Bowen, J. Drelich, J. Goldman, Zinc exhibits ideal physiological corrosion behavior for bioabsorbable stents, Advanced Materials 25(18) (2013) 2577-2582.

[2] E. Mostaed, M. Sikora-Jasinska, J.W. Drelich, M. Vedani, Zinc-based alloys for degradable vascular stent applications, Acta Biomaterialia 71 (2018) 1-23.

[3] G. Katarivas Levy, J. Goldman, E. Aghion, The prospects of zinc as a structural material for biodegradable implants-A review paper, Metals 7(10) (2017) 402.

[4] D. Hernández-Escobar, S. Champagne, H. Yilmazer, B. Dikici, C.J. Boehlert, H. Hermawan, Current status and perspectives of zinc-based absorbable alloys for biomedical applications, Acta Biomaterialia (2019).

[5] G. Li, H. Yang, Y. Zheng, X.-H. Chen, J.-A. Yang, D. Zhu, L. Ruan, K. Takashima, Challenges in the use of zinc and its alloys as biodegradable metals: perspective from biomechanical compatibility, Acta Biomaterialia (2019).

[6] P.K. Bowen, E.R. Shearier, S. Zhao, R.J. Guillory, F. Zhao, J. Goldman, J.W. Drelich, Biodegradable metals for cardiovascular stents: from clinical concerns to recent $\mathrm{Zn}$-Alloys, Advanced healthcare materials 5(10) (2016) 1121-1140.

[7] H. Yang, C. Wang, C. Liu, H. Chen, Y. Wu, J. Han, Z. Jia, W. Lin, D. Zhang, W. Li, Evolution of the degradation mechanism of pure zinc stent in the one-year study of rabbit abdominal aorta model, Biomaterials 145 (2017) 92-105.

[8] R.J. Guillory, P.K. Bowen, S.P. Hopkins, E.R. Shearier, E.J. Earley, A.A. Gillette, E. Aghion, M. Bocks, J.W. Drelich, J. Goldman, Corrosion characteristics dictate the long-term inflammatory profile of degradable zinc arterial implants, ACS Biomaterials Science \& Engineering 2(12) (2016) 2355-2364.

[9] R.J. Guillory II, M. Sikora-Jasinska, J.W. Drelich, J. Goldman, In Vitro Corrosion and In Vivo Response to Zinc Implants with Electropolished and Anodized Surfaces, ACS applied materials \& interfaces (2019).

[10] D. Pierson, J. Edick, A. Tauscher, E. Pokorney, P. Bowen, J. Gelbaugh, J. Stinson, H. Getty, C.H. Lee, J. Drelich, A simplified in vivo approach for evaluating the bioabsorbable behavior of 
candidate stent materials, Journal of Biomedical Materials Research Part B: Applied Biomaterials 100(1) (2012) 58-67.

[11] R.J. Guillory, A.A. Oliver, E.K. Davis, E.J. Earley, J.W. Drelich, J. Goldman, Preclinical In Vivo Evaluation and Screening of Zinc-Based Degradable Metals for Endovascular Stents, JOM 71(4) (2019) 1436-1446.

[12] S. Zhao, J.-M. Seitz, R. Eifler, H.J. Maier, R.J. Guillory II, E.J. Earley, A. Drelich, J. Goldman, J.W. Drelich, Zn-Li alloy after extrusion and drawing: structural, mechanical characterization, and biodegradation in abdominal aorta of rat, Materials Science and Engineering: C 76 (2017) 301-312.

[13] A.J. Drelich, S. Zhao, R.J. Guillory II, J.W. Drelich, J. Goldman, Long-term surveillance of zinc implant in murine artery: Surprisingly steady biocorrosion rate, Acta biomaterialia 58 (2017) 539-549.

[14] H. Jin, S. Zhao, R. Guillory, P.K. Bowen, Z. Yin, A. Griebel, J. Schaffer, E.J. Earley, J. Goldman, J.W. Drelich, Novel high-strength, low-alloys Zn-Mg $(<0.1 \mathrm{wt} \% \mathrm{Mg})$ and their arterial biodegradation, Materials Science and Engineering: C 84 (2018) 67-79.

[15] P.K. Bowen, R.J. Guillory II, E.R. Shearier, J.-M. Seitz, J. Drelich, M. Bocks, F. Zhao, J. Goldman, Metallic zinc exhibits optimal biocompatibility for bioabsorbable endovascular stents, Materials Science and Engineering: C 56 (2015) 467-472.

[16] A.J. Drelich, P.K. Bowen, L. LaLonde, J. Goldman, J.W. Drelich, Importance of oxide film in endovascular biodegradable zinc stents, Surface Innovations 4(3) (2016) 133-140.

[17] E. Mostaed, M. Sikora-Jasinska, A. Mostaed, S. Loffredo, A. Demir, B. Previtali, D. Mantovani, R. Beanland, M. Vedani, Novel Zn-based alloys for biodegradable stent applications: design, development and in vitro degradation, Journal of the Mechanical Behavior of Biomedical Materials 60 (2016) 581-602.

[18] M. Sikora-Jasinska, E. Mostaed, A. Mostaed, R. Beanland, D. Mantovani, M. Vedani, Fabrication, mechanical properties and in vitro degradation behavior of newly developed $\mathrm{ZnAg}$ alloys for degradable implant applications, Materials Science and Engineering: C 77 (2017) 11701181.

[19] E. Mostaed, M.S. Ardakani, M. Sikora-Jasinska, J.W. Drelich, Precipitation induced room temperature superplasticity in Zn-Cu alloys, Materials Letters 244 (2019) 203-206.

[20] P. AshaRani, G. Low Kah Mun, M.P. Hande, S. Valiyaveettil, Cytotoxicity and genotoxicity of silver nanoparticles in human cells, ACS nano 3(2) (2008) 279-290. 
[21] K. Beaussant Törne, F.A. Khan, A. Örnberg, J. Weissenrieder, Zn-Mg and Zn-Ag degradation mechanism under biologically relevant conditions, Surface Innovations 6 (2018) 8192.

[22] G.f. Hu, Copper stimulates proliferation of human endothelial cells under culture, Journal of cellular biochemistry 69(3) (1998) 326-335.

[23] C.W. McCarthy, R.J. Guillory, J. Goldman, M.C. Frost, Transition-metal-mediated release of nitric oxide (NO) from S-nitroso-N-acetyl-d-penicillamine (SNAP): potential applications for endogenous release of $\mathrm{NO}$ at the surface of stents via corrosion products, ACS applied materials \& interfaces 8(16) (2016) 10128-10135.

[24] T.L. Cornwell, E. Arnold, N.J. Boerth, T.M. Lincoln, Inhibition of smooth muscle cell growth by nitric oxide and activation of cAMP-dependent protein kinase by cGMP, American Journal of Physiology-Cell Physiology 267(5) (1994) C1405-C1413.

[25] R. Gryglewski, R. Palmer, S. Moncada, Superoxide anion is involved in the breakdown of endothelium-derived vascular relaxing factor, Nature 320(6061) (1986) 454. 


\section{Zinc Based Materials Regulate the Vascular Biologic Response to Implants by Releasing Therapeutic Transition Metal Ions ${ }^{8}$}

\subsection{Introduction}

Zinc (Zn) based degradable scaffolds under development for cardiovascular applications have garnered increasing attention in recent years [1-5]. Metallurgical innovations that increase the mechanical properties have positioned this class of degradable metals to move forward in preclinical testing of stent prototypes [6]. However, while there has been extensive research and development with regards to alloying and materials refinement, the biological consequences of a degrading zinc-based arterial implant have not been well addressed in both fundamental and clinical studies.

The concept of biodegradation is straightforward; physiological fluids break the material down into byproducts that are either metabolized or cleared from the implant site. As the implant degrades, the space occupied by the material is gradually infiltrated by cells and matrix and reintegrated into the host environment. As with all implanted materials, the initial cells arriving at the material-tissue interface are predominantly macrophages and foreign body giant cells that help clear the implanted material [7]. For arterial implants, smooth muscle cells (SMCs) eventually form a dominant cell type within a developing neointima [8]. For materials that are designed to degrade, an added consideration beyond the original material is the effect of degradation byproducts on the behavior of infiltrating cells. Magnesium (Mg) alloys and polymers such as polylactic acid (PLLA) remain the most widely studied materials for fully degradable stent devices in terms of the biological action of byproducts. Magnesium degrades into $\mathrm{Mg}^{2+}, \mathrm{Mg} / \mathrm{O}$, and $\mathrm{MgOH}_{2}$, eventually leaving behind a $\mathrm{Ca} / \mathrm{P}$ rich product [9-11]. PLLA degrades by hydrolysis into nontoxic biocompatible products [12]. Similar to the selection of bioinert metals for conventional

\footnotetext{
${ }^{8}$ Material in this chapter is in preparation to be submitted to a journal for publication
} 
biostable stents, a primary consideration behind selecting bioresorbable materials for stenting has been to avoid negative biological responses. Indeed, Mg and PLLA materials have advanced through pre-clinical studies and entered into clinical trials in large part because their degradation byproducts do not interfere with the normal functioning of the native surrounding cells and are highly unlikely to promote systemic effects[11-13].

Because the byproducts of degradation can diffuse away from the implant interface, clarifying the biological action of degradation byproducts on vascular smooth muscle cells is of paramount importance. Early work with zinc uncovered $\mathrm{LD}_{50}$ values that were well below that of $\mathrm{Mg}$ and $\mathrm{Fe}$ (iron) $[14,15]$. This raised the prospect that ionic $\mathrm{Zn}$ might exert a strongly toxic effect on arterial cells. However, the exact opposite way of thinking was suggested by early in vivo findings. Low inflammation, the absence of any overt signs of necrosis, and a stable neointima predominated around $\mathrm{Zn}$ implanted into rat arteries out to $\sim 6$ months[16]. Based on the in vitro findings, one would have expected to see a strong inflammatory response, the clear presence of necrotic regions, and a negative neointimal progression. Together, the in vitro and in vivo findings suggest that the biological effects of zinc on neointimal progression may not be mediated by toxicity, but rather by programmed intracellular signaling pathways.

Due to zinc's inherently low mechanical properties, a Zn stent material requires the addition of alloying elements[1-5]. Alloying of zinc with non-toxic elements at their specified concentrations can be used to improve mechanical properties and refine microstructure of the implant. If an element exerts beneficial bioactive effects on arterial cells, its incorporation into the bulk matrix ensures its elution as the implant degradation progresses. In such a manner, an advanced bioresorbable stent metal can be engineered to release multiple beneficial bioactive elements at optimized concentrations for the lifetime of the implant. Alloying with copper, for instance, can improve the structural and mechanical properties of zinc implants[17]. In this communication, we introduce a new concept of using $\mathrm{Zn}^{2+}$ and $\mathrm{Cu}^{2+}$ ions as potential therapeutic elutants that are supplied to a host site from degradable zinc-based implant. 


\subsection{Experimental}

\subsubsection{Materials}

ACS reagent grade methanol was purchased from Millipore Sigma (St. Louis, MO). Bradford assay kit (ab102535), caspase-3,caspase- 8 and caspase- 9 multiplex activity assay kit fluorometric (ab219915), TUNEL assay kit (ab206386), anti-cleaved caspase-3 antibody (ab2302), anti-alpha smooth muscle actin antibody (ab5694), and goat antirabbit IgG Alexa Fuor 488 were all purchased from Abcam (Cambridge, MA). 4',6Diamidino-2-phenylindole dihydrochloride (DAPI) (D8417), Dulbecco's modified eagles medium-low glucose (DMEM) (D6046), Dulbecco's phosphate buffered saline (PBS) (D8662), penicillin-streptomycin (P4333), goat serum (G9023), zinc acetate (383317), D-glucose(G8270), and fetal bovine serum (F2442) were purchased from Sigma-Aldrich ( St. Louis, MO). 3-morpholin-4-ylpropane-1-sulfonic acid (MOPS)(172630250) was purchased from Fisher Scientific ( Toronto, ON). High purity Zn wires (4N-99.99wt\%) were purchased from Goodfellow (Coraopolis, PA) Platinum wires were purchased from Fort Wayne Metals (IN).

\subsubsection{Methods}

\subsubsection{In vivo Implantation}

Pure zinc $(\mathrm{Zn})$ and platinum $(\mathrm{Pt})$ wires were implanted into the abdominal aorta of adult female Sprague Dawley rats for 3 months, $n=5$. A detailed description of the surgical procedure was provided in our earlier publication [18]. The wire and tissue explants were collected, surrounded by optimal cutting media, and snap frozen in liquid $\mathrm{N}_{2}$. The samples were stored at $-80^{\circ} \mathrm{C}$ until cryo-sectioning. 


\subsubsection{General Histology, immunofluorescence and in-situ apoptosis detection}

Wire/tissue samples were cross-sectioned with a cryo-microtome at $10 \mu \mathrm{m}$ section thickness and placed on histobond glass slides. Multiple sections spanning a $\sim 0.2 \mathrm{~mm}$ distance were collected for each sample. For hematoxylin and eosin staining (H\&E) and TUNEL labeling, sections were fixed in paraformaldehyde for $10 \mathrm{~min}$. H\&E staining was carried out by protocols described by the authors previously $[18,19]$, and TUNEL labeling was performed following manufactures' protocols.

For immunofluorescence, sections were fixed in ice cold methanol for $5 \mathrm{~min}$ and allowed to air dry before washing. The slides were rinsed $3 \mathrm{X}$ in PBS, then blocked in a $10 \%(\mathrm{v} / \mathrm{v})$ goat serum solution in PBS for 30 min. After blocking, slides were either incubated with an anti-cleaved caspase-3 antibody (1/100 dilution) overnight at $4{ }^{\circ} \mathrm{C}$, or an anti- smooth muscle alpha actin antibody (1/250 dilution) for $1 \mathrm{hr}$ at room temperature (RT). After incubation, the slides were rinsed $3 \mathrm{x}$ with $\mathrm{PBS}$ and incubated with goat anti-rabbit $\operatorname{IgG}$ Alexa fluor 488 (1/300 dilution) for $1 \mathrm{hr}$ at RT. The secondary solution was rinsed with PBS $3 x$, then stained with a DAPI solution (1/1000 dilution of $1 \mathrm{mg} / \mathrm{mL}$ stock) for $2 \mathrm{~min}$ at RT. DAPI was rinsed off with PBS and the slides were mounted with a glass coverslip and aqueous mounting media, and imaged immediately with an upright BX51 Olympus epifluorescence microscope.

\subsubsection{Ex-vivo arterial ring tissue culture}

In order to investigate the potential of $\mathrm{Zn}^{2+}$ to activate caspase-3 in smooth muscle cells directly, an ex vivo arterial culture model was developed. The thoracic aorta was collected from donor adult female Sprague Dawley rats. Each thoracic aorta was cut into rings of approximately 5-8 mm in length in Dulbeco's PBS supplemented with $1 \%$ penicillin-streptomycin (PS) (v/v). The arterial rings were randomized and allowed to recover overnight in low glucose DMEM supplemented with $10 \% \mathrm{FBS}$ and $1 \% \mathrm{PS}$, at 5\% $\mathrm{CO}_{2}$ and $37^{\circ} \mathrm{C}$. After overnight recovery (to allow cells to adjust to culture conditions and recover from injury), the arterial rings were randomly immersed in a physiological saline solution (131 mM NaCl, $4 \mathrm{mM} \mathrm{KCl}, 2.5 \mathrm{mM} \mathrm{CaCl}_{2}$, $5.5 \mathrm{mM}$ glucose, and $1 \mathrm{mM} \mathrm{MgCl}_{2}$ ) 
buffered by $10 \mathrm{mM}$ MOPS at a $\mathrm{pH}$ of 7.25 (pH adjusted with sodium hydroxide), or the same physiological saline solution with the addition of $0.5 \mathrm{mM} \mathrm{Zn}$ acetate, $\mathrm{MgCl}_{2}, \mathrm{FeCl}_{3}$, or $\mathrm{CuCl}_{3}$. The arterial rings were incubated in the control and insult loaded saline solutions for 12 hours.

After incubation, the smooth muscle cell containing media compartment was mechanically separated from the other arterial compartments. The adventitia compartment for each arterial ring was bluntly dissected from the media layer using forceps under a stereomicroscope. Removal of the endothelial cells was confirmed with en-face DAPI imaging (not shown), and the media layer was immediately washed in PBS containing no magnesium and calcium and stored in lysis buffer at $-80^{\circ} \mathrm{C}$ for further processing.

\subsubsection{Multi-caspase activity assay}

Caspase- 3 , caspase-8, and caspase- 9 activity was detected for the media compartment of the arterial rings using a plate based fluorescent assay. The compartment was homogenized in a lysis buffer provided by the manufacturer, and protein determination was carried out using a Bradford assay kit. The multiplex caspase activity kit (abcam ab219915) provides each substrate used to detect its respective caspase in a conjugated form to fluorophores with wide spectral separation, allowing multi-caspase activity to be determined in the same lysate. There was a minimum of 4 samples per condition $(n=4)$.

\subsubsection{Confocal microscopy}

Confocal microscopy was used to inspect the in situ localization of active caspase-3 within the zinc-insulted arterial segments. After collection, thoracic aortas were allowed to recover in supplemented DMEM overnight at $37^{\circ} \mathrm{C}$. The segments were then rinsed in PBS and incubated in either a $0.5 \mathrm{mM}$ zinc solution or control saline solution. The segments were then rinsed in PBS, surrounded by optimal cutting medium and snap frozen in liquid $\mathrm{N}_{2} .50 \mu \mathrm{m}$ thick cryosections were taken at five evenly spaced locations. These sections were stained for active caspase- 3 as described above, imaging three 
locations per section with an Olympus FluoViewTM FV1000 laser scanning confocal microscope. $30 \mu \mathrm{m}$ volume stacks were taken for each 600x magnified field.

\subsubsection{Corrosion of alloys and ICP-OES analysis}

Ternary $\mathrm{Zn}-4 \mathrm{Ag}-0.6 \mathrm{Mn}$ and quinary $\mathrm{Zn}-4 \mathrm{Ag}-0.8 \mathrm{Cu}-0.6 \mathrm{Mn}-0.15 \mathrm{Zr}$ ultrafine zinc wires were corroded in Hanks balanced salt solution [20] for 14 and 28 days. The solutions supernatant were then sterile filtered with a $0.22 \mu \mathrm{m}$ syringe filter. After filtration, the solutions were acid digested with trace metal basis nitric acid, and analyzed using inductively coupled optical emission spectroscopy (ICP-OES). The detection limit for copper is $10 \mathrm{ppb}$.

\subsubsection{Statistical Methods}

An unpaired two sample student's $t$ test with unequal variance was used to evaluate experimental groups. Error bars are given as sample standard error. A minimum of 4 sample replicates $(\mathrm{n}=4)$ was included for all experimental groups. A one-way ANOVA was used to compare multiple groups. 


\subsection{Results}

\subsubsection{Morphometric presentation}

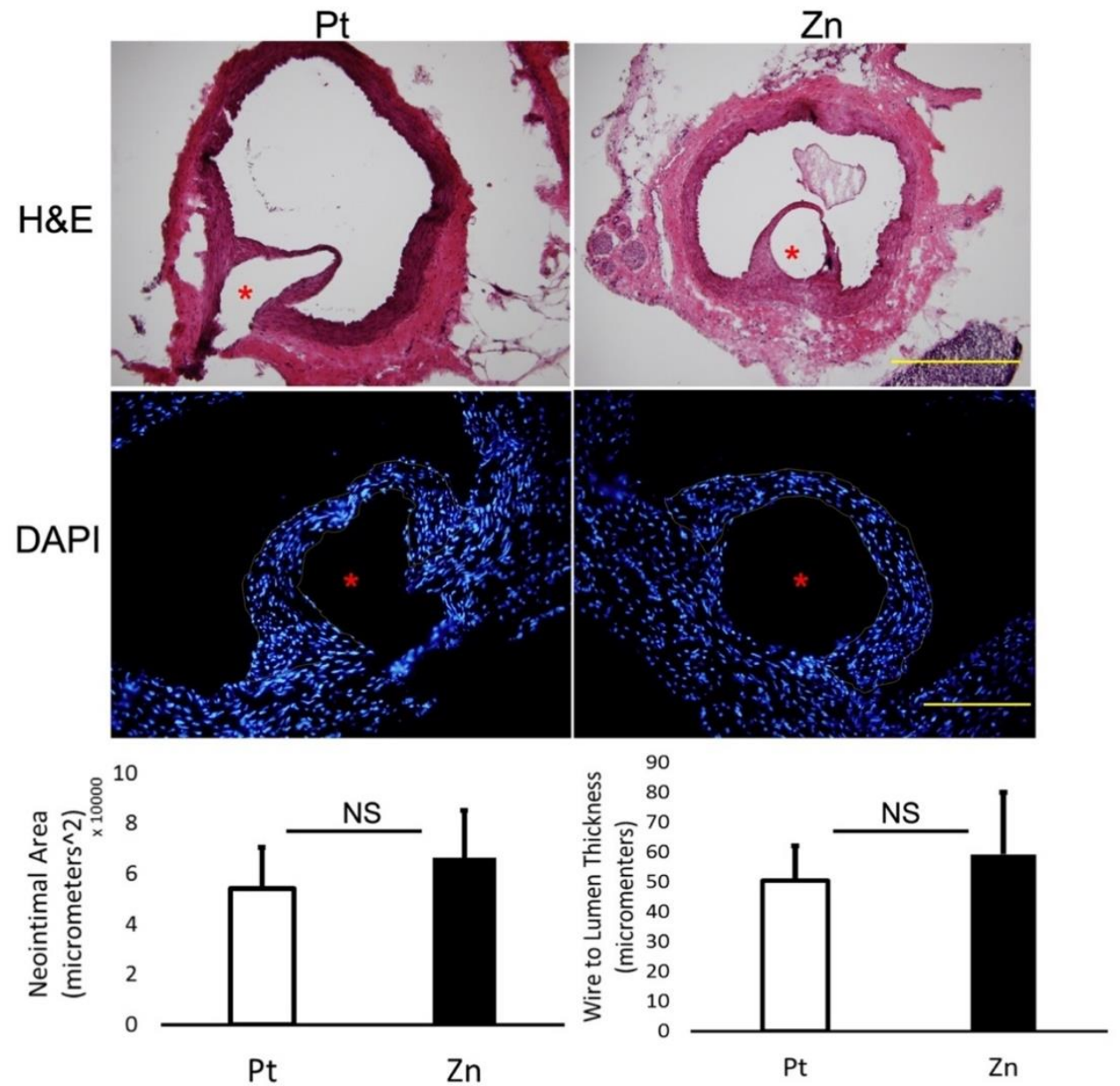

Fig.5-1 General histomorphometry and neointimal cellularization of Pt and Zn The typical appearance of the neointimas (NI) that developed on $\mathrm{Zn}$ and Pt wires is shown in Fig.5-1. H\&E staining at 100x normal magnification depicts thin, continuous NI formation over both wires. In order to compare the cellularity of the NI produced by the two materials, DAPI staining was performed, as shown in the bottom two panels of Fig.1. Both NIs appear to be cellularized with a similar spatial distribution of cells. Furthermore, histomorphometric parameters, including NI area for Pt vs. Zn, $(5.4 \pm 1.6) \times 10^{4} \mu \mathrm{m}^{2}$ vs. $(6.6 \pm 1.9) \times 10^{4} \mu \mathrm{m}^{2}$ and 
wire to lumen thickness ( $50 \pm 12 \mu \mathrm{m}$ vs. $59 \pm 21 \mu \mathrm{m}$ ) show a slight but nonsignificant elevation for $\mathrm{Zn}(\mathrm{p}=0.308$ and $p=0.433$, for area and thickness, respectively) ( $n=5$ per condition).

\subsubsection{TUNEL labeling of $\mathrm{NI}$ and $\alpha-\mathrm{SM} /$ active caspase-3 presence}

Cross sections of the Pt and $\mathrm{Zn}$ implants were labeled for TUNEL+ identification. The Pt specimens displayed low TUNEL+ staining in both the luminal and mural regions of the NI. In stark contrast, all Zn specimens demonstrated strong TUNEL+ staining within both the mural and luminal portions of the NI. When taken as a percentage of area covered by threshold analysis, the NI surrounding $\mathrm{Zn}$ implants was significantly more TUNEL $+(2.4 \pm 2.1 \%$ vs $6.5 \pm 1.5 \%, p=0.008, P t n=5$ vs $Z n n=5$, respectively).

In addition to higher TUNEL + staining within the NI surrounding Zn implants, we observed a reduced amount of $\alpha$-SMA area coverage in Zn NI tissue compared to the platinum control ( $23 \pm 2 \%$ vs $14 \pm 6 \%$, p $=0.017$, Pt vs $Z n$, respectively). This reduced amount of $\alpha$-SMA area coverage for $\mathrm{Zn}$ specimens was associated with an increased amount of area coverage in the NI for active caspase-3 $(4.3 \pm 2.7 \%$ vs. $8.6 \pm 2.0 \%$, $\mathrm{p}=0.032, \mathrm{Pt} \mathrm{n}=5$ vs $\mathrm{Zn} \mathrm{n}=4$ respectively). 

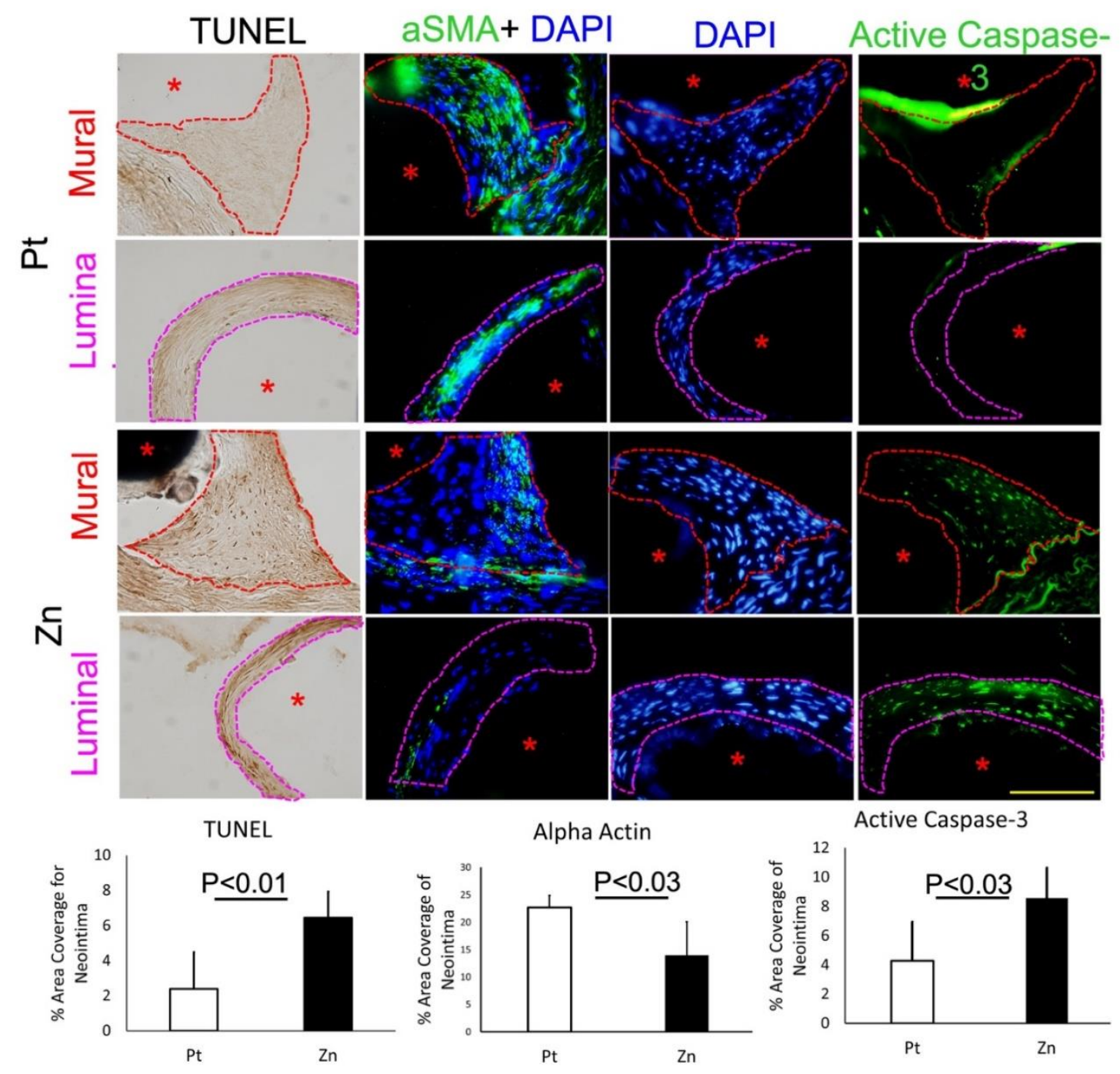

Fig.5-2 Neointima cellular and protein expression of Pt and Zn. TUNEL, alpha smooth muscle actin, active caspase 3 and DAPI staining were performed for a minimum 3 sections per sample ( $n=5$ samples per condition). Data error is reported in standard sample error and statistical significance is shown by a Welch's T test. Scale bar is set at approximately $100 \mu \mathrm{m}$. 


\subsubsection{Dose dependent caspase $-3,-8$, and -9 activation by $\mathrm{Zn}$}

In order to determine whether $\mathrm{Zn}$ may promote caspase activation in smooth muscle cells, we incubated arterial segments in multiple concentrations of $\mathrm{Zn}$ for 12 hours, ex vivo. We found that smooth muscle cell caspase-9 is not activated by $0.5 \mathrm{mM} \mathrm{Zn} \mathrm{(Fig} \mathrm{5-3a).}$ Interestingly, higher $\mathrm{Zn}$ concentrations significantly suppress caspase-9 activity from basal levels ( $1 \mathrm{mM}$ and $1.5 \mathrm{mM}, \mathrm{p}<0.00005)$. An increase in caspase- 8 activity is detected at $0.5 \mathrm{mM}$ relative to the control condition, Fig.5-3b ( $p<0.0005)$. Higher concentrations of zinc significantly inhibit caspase- 8 relative to the $0.5 \mathrm{mM}$ condition (1 $\mathrm{mM}$ and $1.5 \mathrm{mM}, \mathrm{p}<0.00005)$. Caspase- 3 activity was significantly elevated at all treatment conditions (Fig. 3c, $\mathrm{p}<0.05$ at $0.5 \mathrm{mM}, \mathrm{p}<0.0005$ at $1 \mathrm{mM}$, and $\mathrm{p}<0.005$ at 1.5 $\mathrm{mM}$ ) relative to the control group. Activity significantly increased from $0.5 \mathrm{mM}$ to $1 \mathrm{mM}$ and from $0.5 \mathrm{mM}$ to $1.5 \mathrm{mM}$ ( $\mathrm{p}<0.05)$. Therefore caspase- 3 activity was not suppressed by any of the zinc concentrations evaluated.

\subsubsection{Effect of transition metal ions on caspase activity}

In order to investigate whether the transition metal ions of common degradable metals stimulate caspase activity at excess concentration, we performed activity assays with arteries that were incubated in $0.5 \mathrm{mM}$ solutions of either $\mathrm{Fe}, \mathrm{Mg}$, or $\mathrm{Zn}$. Zn elicits the strongest increase of caspase- 3 activity as compared to $\mathrm{Mg}$ and Fe transition metal ions (Fig.5-3.D, $\mathrm{p}<0.0005$ ). 

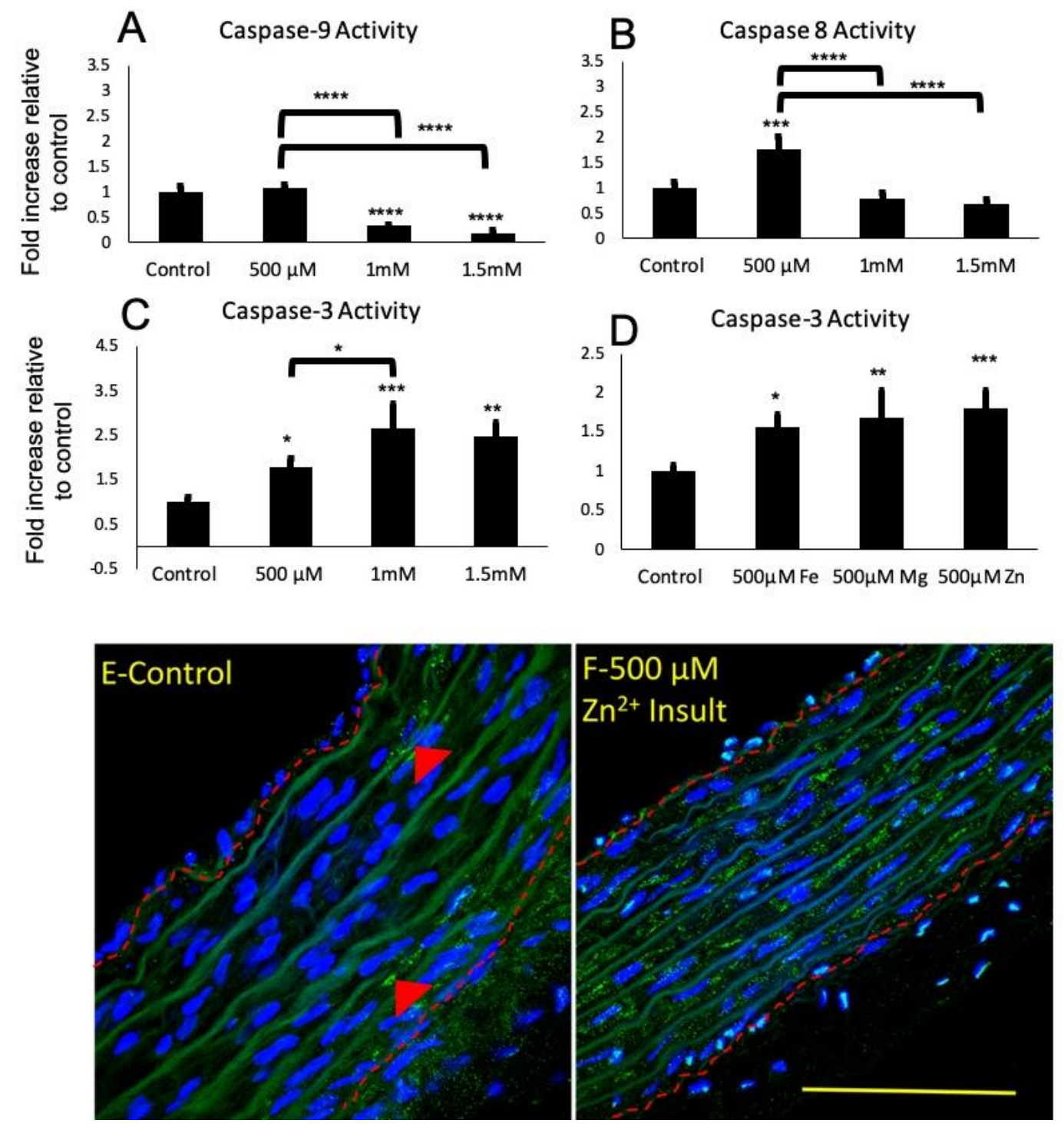

Fig.5-3 Caspase activation in the ex vivo arterial culture system. Caspase -9 (A), $-8(B)$, and $-3(C)$ activity in 3 different concentrations is shown. Caspase-3 activation in $500 \mu \mathrm{M} \mathrm{Fe}, \mathrm{Mg}$, and $\mathrm{Zn}$ is shown in (D). E and $\mathrm{F}$ represent confocal images from control and $\mathrm{Zn}$ treatment groups respectfully of active caspase 3 staining present in cross-sections. Data error is presented in sample standard error and $*=p<0.05, * * p<0.005, * * * p<0.0005$, and $* * * * p<0.00005$. 


\subsubsection{Addition of $\mathrm{Cu}$ to bulk zinc materials}

A promising $\mathrm{Zn}$ material alloyed with copper $(\mathrm{Cu})$ was selected to evaluate the potential biological effect of its eluted $\mathrm{Cu}$ constituent on caspase activity. Fig.5-4 shows the neointimal response to $\mathrm{Zn}-4 \mathrm{Ag}-0.6 \mathrm{Mn}$, and $\mathrm{Zn}-4 \mathrm{Ag}-0.8 \mathrm{Cu}-0.6 \mathrm{Mn}-0.15 \mathrm{Zr}$ alloy wires implanted within the abdominal aorta for up to 6 months. The mural (Fig.5-4a) and luminal (Fig.5-4b) portions of Zn-4Ag-0.6Mn wire neointimas (outlined by the blue arrows) failed to endothelialize. In contrast, a stable endothelium is clearly seen for the $\mathrm{Zn}-4 \mathrm{Ag}-0.8 \mathrm{Cu}-0.6 \mathrm{Mn}-0.15 \mathrm{Zr}$ wires (Fig. $5-4 \mathrm{C}$ and D - identified by the red arrows).

ICP analysis in Fig. 5-4E shows the elemental profile of in vitro corrosion fluid for the two respective alloys at 14 and 28 days. Significant $\mathrm{Cu}$ release can be seen for the $\mathrm{Cu}$ containing alloy, with no $\mathrm{Cu}$ release for the $\mathrm{Cu}$-free control (red line denotes detection limit for the ICP system to $\mathrm{Cu}$ ). The ICP results demonstrate the release of $\mathrm{Cu}$ ions into the corrosion fluid from the $\mathrm{Cu}$-containing alloy. To evaluate whether $\mathrm{Cu}$ could induce caspase activity, we incubated arterial segments in $5 \mu \mathrm{M}$ and $50 \mu \mathrm{M} \mathrm{Cu}$ solutions for 12 hours and probed for caspase-3 activity. Neither condition elicited significant caspase activity within smooth muscle cells, indicative of caspase-independent mechanisms of biologic interaction. 

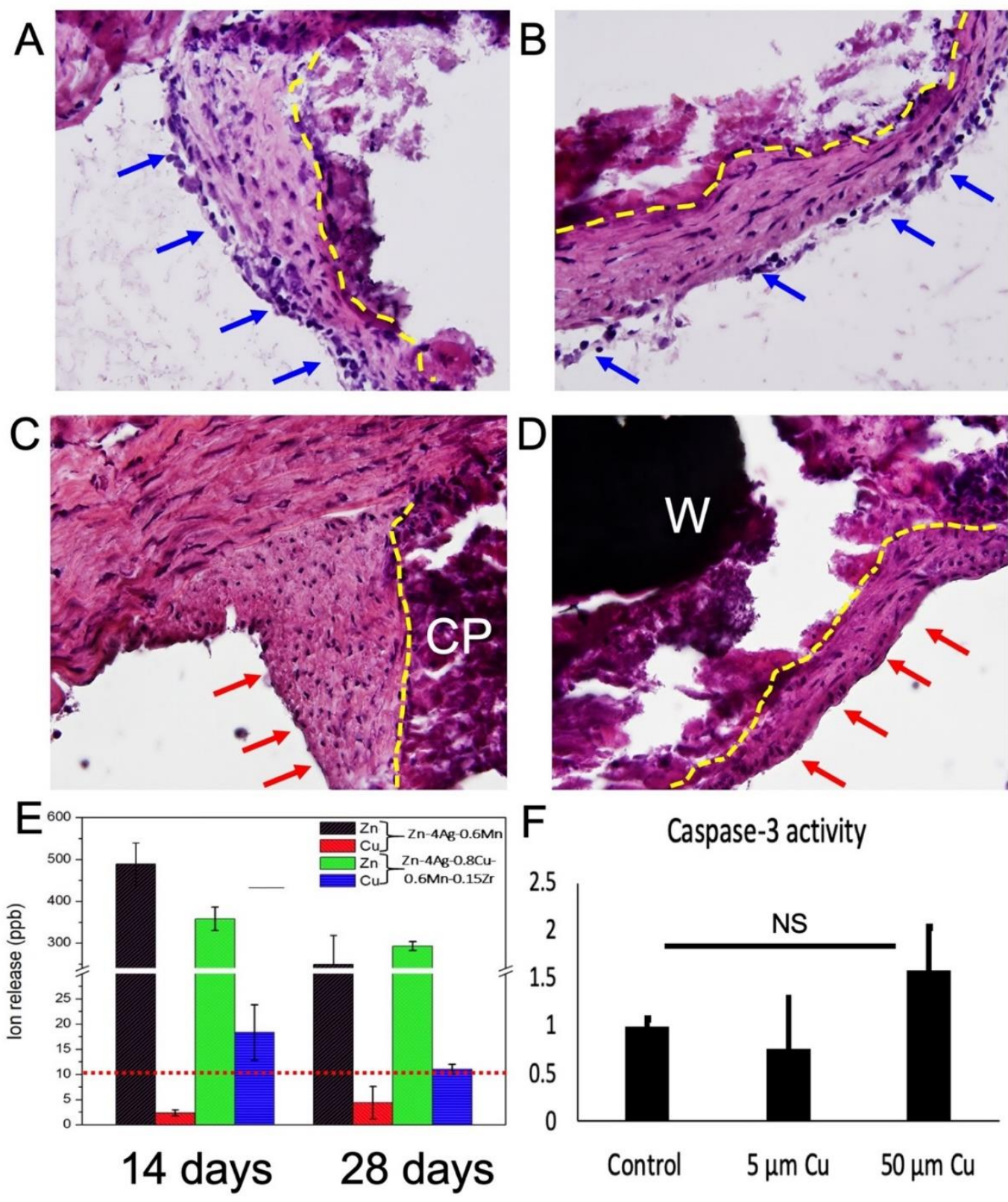

Fig.5-4 Copper addition in advance zinc alloys effects the vascular response through non caspase dependent pathways. A and B show mural and luminal 600X normal magnification H\&E images of $\mathrm{Zn}-4 \mathrm{Ag}-0.6 \mathrm{Mn}$ wires at 6 months within the abdominal aorta. Blue arrows depict inflamed endothelium. $C$ and $D$ 
show luminal and mural views $\mathrm{Zn}-4 \mathrm{Ag}-0.8 \mathrm{Cu}-0.6 \mathrm{Mn}-0.15 \mathrm{Zr}$ wires at 6 months in histological cross-section. Red arrows show confluent, non-inflamed endothelium. E shows ICP analysis of corrosion media

\subsection{Discussion}

The present study explored the similarities and differences between the neointimas that develop around degradable (zinc) and non-degradable (platinum) implant metals. From a histomorphometric perspective, platinum and zinc materials placed into healthy murine arteries form neointimas with similar characteristics (Fig.5-1). From a broad view, the similar cell densities, neointimal area, and neointimal thickness measurements demonstrate the promise for use of zinc-based metals as vascular implants.

Materials selected in the past to serve as stents, either as biostable or bioresorbable materials, are intended to be highly bioinert in order to avoid interaction with local and systemic systems. Although these materials perform superbly when tested in healthy animal arteries, they often fail to prevent progressive intimal hyperplasia and restenosis when deployed into the atherogenic environment of diseased human arteries. The limitations of this approach have necessitated the incorporation of drug-eluting coatings, in an effort to impart suppressive properties to bioinert stent materials.

Here we show that zinc ions elution from metallic zinc platforms potently suppresses neo-intimal hyperplasia of smooth muscle cells (SMCs), widely recognized as the primary cause of failure for stented human arteries [21-23]. We have recently reported on the promising in vivo behavior of metallic zinc implants in the abdominal aorta of Sprague Dawley rats, out to 6 months [16]. Confluent endothelialization was found along the luminal surface of the stable neointima, with a decreasing gradient of smooth muscle cells (SMCs) close to the implant surface [16]. Based on these preliminary observations, we hypothesized that the byproducts of zinc corrosion may exert suppressive effects on arterial SMCs. The present findings implicating caspase enzymatic activity and apoptosis signaling pathways in mediating the suppressive effects strongly suggest that the 
response to ionic zinc is programmed, rather than a response to injury. If zinc ions were toxic to SMCs, we would have observed inflammation, necrosis, and progressive intimal hyperplasia.

Zinc is a well-known inhibitor of caspase-3, 8, and 9 activity when evaluated in concentrations exceeding $100 \mu \mathrm{M}$ [24-26]. Although this inhibitory effect has been described extensively in literature using cell-free systems [26], the relationship between excess zinc and caspase enzymatic activity using intact cell systems is less clear. For instance, it has been shown that excess zinc at high concentrations can provoke mixed forms of cell death in cancer cell lines, mediated by caspase cascade activation [27] . However, studies focusing on the effects excess zinc in vascular smooth muscle cells is relatively lacking. Because the concentration of zinc is expected to be high directly at the interface of a zinc-based implant, we sought to measure caspase activity in neointimal cells at the interface of zinc wires implanted into the arterial environment and in arterial media layers cultured ex vivo in simulated high zinc environments.

Caspases 3 and 8, but not 9 become significantly elevated in high concentration zinc conditions, as shown by Fig.5-3. Surprisingly, at concentrations of zinc exceeding 0.5 $\mathrm{mM}$, caspases 8 and 9 dramatically decrease in activity relative to both the control and $0.5 \mathrm{mM}$ conditions. Conversely, caspase- 3 continues to increase in activity with zinc concentration, consistent with the extensive apoptosis we have detected in the neointimas of zinc wires. This is also not surprising, as caspase- 3 is the most robust caspase in respect to inhibition of the proteolytic activity, requiring $1 \mathrm{mM}$ for complete inhibition of the enzyme [26]. Caspase-9 is known to be potently inhibited by zinc [24], which could explain its lack of activation in the lower concentration condition and suppressed activity at the higher zinc levels. Caspase- 8 may also be inhibited in some manner at high concentrations of ionic zinc. The data suggests a complex interplay of caspase activation and zinc concentration, which ultimately yields caspase- 3 activation and apoptosis execution. 
We also found an anti-inflammatory effect from the added elution of copper ions, which significantly reduced neointimal thickness and inflammation around the implant. This effect is independent of the caspase-3 induced activation of zinc, as shown in Fig.5-4f. It has been reported that both $\mathrm{Zn}^{2+}$ and $\mathrm{Cu}^{2+}$ can stimulate NO release from endogenous and synthetic S-nitrosothiols (RSNOs) [28-30]. We recently reported that the amount of NO released by $\mathrm{Cu}^{2+}$ from the synthetic RSNO, SNAP, was an order of magnitude higher than that of $\mathrm{Zn}^{2+}[31]$. Free endogenous RSNOs circulate in the blood and are constantly replenishing [32], providing a continuous source of $\mathrm{NO}$ generation for degradable materials that elute $\mathrm{Zn}^{2+}$ and/or $\mathrm{Cu}^{2+}$. Local $\mathrm{NO}$ production from the release of $\mathrm{Cu}^{2+}$ from biodegradable materials in addition to $\mathrm{Zn}^{2+}$ may improve the biological response, owing to the critical physiological roles of NO in regulating vascular function. Beneficial effects of NO include inhibition of platelet aggregation [33], leukocyte adhesion [34, 35], and vascular SMC proliferation [36], all contributing factors to the restenosis of stented arteries. Interestingly, it has been shown in peripheral blood mononuclear cells that zinc protects against copper induced DNA damage, and substantially raises the LD50 value of copper [37]. Copper also increases the proliferation rate of endothelial cells, but not SMC's [38].Fig.5-4 nicely shows a confluent, endothelium that is largely lacking in the inflammatory ridden ternary alloy.

The present findings have major implications for the future of bioresorbable stent development. Drug eluting polymer coated stents (DES) have become the main approach for the revascularization of occluded coronary arteries. These stents combine mechanical scaffolding with the release of pharmaceutical agents from a polymer coating on the stent surface that act to inhibit smooth muscle cell proliferation [39]. Although second generation DES have reduced the rates of late stage thrombosis relative to bare metal stents (BMS) [39], these stents still experience a $\sim 10 \%$ incidence of in-stent restenosis (ISR) [40]. Furthermore, ISR for DES begins after $6-9$ months and increases up to 2 years following implantation, as opposed to peaking between $3-6$ months for BMS [40, 41]. The time course suggests limitations related to the drug in addition to the inherent limitations of permanent stents. For instance, the drug release time course is limited to 
several months. Of potentially major importance when considering drug safety, paclitaxel has recently been shown to sharply increase the risk of death many years after stent deployment, likely due to systemic side effects [42]. It is an open question whether other commonly used drugs for stent elution elicit similar systemic effects.

Recognized limitations with DESs have given rise to the concept of fully bioresorbable polymeric scaffolds (BRS), which are in ongoing development to replace their permanent metallic counterparts [43]. This feature is expected to restore positive arterial remodeling while removing sources of thrombogenesis, inflammation, and neointimal activation. However, all the polymers that have been selected for scaffolding exhibit substantially reduced mechanical properties relative to metals, necessitating thicker struts. This worsens malapposition, reduces endothelial regeneration, and leads to an increased risk of thrombosis [43]. Bioresorbable metallic alternatives are further behind in development, yet with appropriate modification can achieve similar mechanical properties as conventional stent metals and have achieved some preliminary success [43]. Unfortunately, $\mathrm{Fe}$ and $\mathrm{Mg}$ based scaffolds will require drug eluting polymer coatings when deployed into small diameter arteries.

Due to the limitations of anti-proliferative eluting drugs, the development of a bioresorbable metallic stent that does not require synthetic drug elution is urgently needed. In marked contrast to what has been shown previously for $\mathrm{Fe}$ and $\mathrm{Mg}$, we have shown here that the byproducts of zinc implant biocorrosion suppress the harmful activities of neo-intimal smooth muscle cells. $\mathrm{Zn}^{2+}$ and $\mathrm{Cu}^{2+}$, like $\mathrm{Mg}^{2+}$ and $\mathrm{Fe}^{2+}$, are present in the body naturally and the low quantities released from a stent that biodegrades over a year, in particular with copper included as a low concentration alloying addition, are unlikely to substantially alter their systemic levels. Thus, $\mathrm{Zn}^{2+}$ and $\mathrm{Cu}^{2+}$ may act in a similar fashion as synthetic eluting drugs to protect against intimal hyperplasia of smooth muscle cells, yet for a longer duration and without the harmful systemic side effects of synthetic drugs. We propose, consequently, that the development of bioresorbable metallic zinc stents could proceed without polymer coatings or synthetic drug elution. 


\subsection{Conclusions}

In the present paradigm, byproducts of implant degradation are seen largely as systemic pollutants to be cleansed from the body without promoting toxic effects. We propose a new paradigm, wherein the appropriate degradation byproducts are seen as therapeutic agents, similar to the synthetic drugs impregnated into modern day drug-eluting stents, yet naturally found in the body. Due to the physiological presence of the element, an elevated local concentration can be achieved to deliver therapy near the implant without promoting systemic effects. It is straightforward to embed multiple components within the zinc matrix and control their concentrations through conventional alloying approaches. Such an approach would serve both to improve the structural and mechanical properties of the material and to elute multiple therapeutic agents into the local environment.

\subsection{Acknowledgments}

Research reported in this publication was supported by the U.S. National Institute of Health—National Institute of Biomedical Imaging and Bioengineering, grant \#R21 EB 024034-01A1 and \#1R21EB019118-01A1. The content is solely the responsibility of the authors and does not necessarily represent the official views of the National Institutes of Health. R.J.G. was supported by the National Science Foundation Graduate Research Fellowship.

\subsection{References}

[1] P.K. Bowen, E.R. Shearier, S. Zhao, R.J. Guillory, F. Zhao, J. Goldman, J.W. Drelich, Biodegradable metals for cardiovascular stents: from clinical concerns to recent Zn-Alloys, Advanced healthcare materials 5(10) (2016) 1121-1140.

[2] D. Hernández-Escobar, S. Champagne, H. Yilmazer, B. Dikici, C.J. Boehlert, H. Hermawan, Current status and perspectives of zinc-based absorbable alloys for biomedical applications, Acta Biomaterialia (2019). 
[3] G. Katarivas Levy, J. Goldman, E. Aghion, The prospects of zinc as a structural material for biodegradable implants-A review paper, Metals 7(10) (2017) 402.

[4] G. Li, H. Yang, Y. Zheng, X.-H. Chen, J.-A. Yang, D. Zhu, L. Ruan, K. Takashima, Challenges in the use of zinc and its alloys as biodegradable metals: perspective from biomechanical compatibility, Acta biomaterialia (2019).

[5] E. Mostaed, M. Sikora-Jasinska, J.W. Drelich, M. Vedani, Zinc-based alloys for degradable vascular stent applications, Acta Biomaterialia 71 (2018) 1-23.

[6] H. Yang, C. Wang, C. Liu, H. Chen, Y. Wu, J. Han, Z. Jia, W. Lin, D. Zhang, W. Li, Evolution of the degradation mechanism of pure zinc stent in the one-year study of rabbit abdominal aorta model, Biomaterials 145 (2017) 92-105.

[7] Z. Xia, J.T. Triffitt, A review on macrophage responses to biomaterials, Biomedical materials 1(1) (2006) R1.

[8] H.-z. Bai, J. Masuda, Y. Sawa, S. Nakano, R. Shirakura, Y. Shimazaki, J. Ogata, H. Matsuda, Neointima formation after vascular stent implantation. Spatial and chronological distribution of smooth muscle cell proliferation and phenotypic modulation, Arteriosclerosis and thrombosis: a journal of vascular biology 14(11) (1994) 1846-1853.

[9] P.K. Bowen, J. Drelich, J. Goldman, Magnesium in the murine artery: Probing the products of corrosion, Acta biomaterialia 10(3) (2014) 1475-1483.

[10] F. Witte, N. Hort, C. Vogt, S. Cohen, K.U. Kainer, R. Willumeit, F. Feyerabend, Degradable biomaterials based on magnesium corrosion, Current opinion in solid state and materials science 12(5-6) (2008) 63-72.

[11] P. Zartner, M. Buettner, H. Singer, M. Sigler, First biodegradable metal stent in a child with congenital heart disease: evaluation of macro and histopathology, Catheterization and Cardiovascular Interventions 69(3) (2007) 443-446.

[12] S. Nishio, K. Kosuga, K. Igaki, M. Okada, E. Kyo, T. Tsuji, E. Takeuchi, Y. Inuzuka, S. Takeda, T. Hata, Long-term ( $>10$ years) clinical outcomes of first-in-human biodegradable poly1-lactic acid coronary stents: Igaki-Tamai stents, Circulation 125(19) (2012) 2343-2353.

[13] R. Erbel, C. Di Mario, J. Bartunek, J. Bonnier, B. de Bruyne, F.R. Eberli, P. Erne, M. Haude, B. Heublein, M. Horrigan, Temporary scaffolding of coronary arteries with bioabsorbable magnesium stents: a prospective, non-randomised multicentre trial, The Lancet 369(9576) (2007) $1869-1875$.

[14] E.R. Shearier, P.K. Bowen, W. He, A. Drelich, J. Drelich, J. Goldman, F. Zhao, In vitro cytotoxicity, adhesion, and proliferation of human vascular cells exposed to zinc, ACS biomaterials science \& engineering 2(4) (2016) 634-642.

[15] F. Feyerabend, J. Fischer, J. Holtz, F. Witte, R. Willumeit, H. Drücker, C. Vogt, N. Hort, Evaluation of short-term effects of rare earth and other elements used in magnesium alloys on primary cells and cell lines, Acta biomaterialia 6(5) (2010) 1834-1842.

[16] P.K. Bowen, R.J. Guillory, 2nd, E.R. Shearier, J.M. Seitz, J. Drelich, M. Bocks, F. Zhao, J. Goldman, Metallic zinc exhibits optimal biocompatibility for bioabsorbable endovascular stents, Mater Sci Eng C Mater Biol Appl 56 (2015) 467-72. 
[17] E. Mostaed, M.S. Ardakani, M. Sikora-Jasinska, J.W. Drelich, Precipitation induced room temperature superplasticity in Zn-Cu alloys, Materials Letters 244 (2019) 203-206.

[18] R.J. Guillory, A.A. Oliver, E.K. Davis, E.J. Earley, J.W. Drelich, J. Goldman, Preclinical In Vivo Evaluation and Screening of Zinc-Based Degradable Metals for Endovascular Stents, JOM 71(4) (2019) 1436-1446.

[19] R.J. Guillory, P.K. Bowen, S.P. Hopkins, E.R. Shearier, E.J. Earley, A.A. Gillette, E. Aghion, M. Bocks, J.W. Drelich, J. Goldman, Corrosion characteristics dictate the long-term inflammatory profile of degradable zinc arterial implants, ACS Biomaterials Science \& Engineering 2(12) (2016) 2355-2364.

[20] R.J. Guillory II, M. Sikora-Jasinska, J.W. Drelich, J. Goldman, In Vitro Corrosion and In Vivo Response to Zinc Implants with Electropolished and Anodized Surfaces, Acs Appl Mater Inter (2019).

[21] M.F. Brancati, F. Burzotta, C. Trani, O. Leonzi, C. Cuccia, F. Crea, Coronary stents and vascular response to implantation: literature review, Pragmat Obs Res 8 (2017) 137-148.

[22] Z.Z. Dai, G.L. Xu, Restenosis after carotid artery stenting, Vascular 25(6) (2017) 576-586.

[23] S.J. Park, S.J. Kang, R. Virmani, M. Nakano, Y. Ueda, In-Stent Neoatherosclerosis A Final Common Pathway of Late Stent Failure, J Am Coll Cardiol 59(23) (2012) 2051-2057.

[24] K.L. Huber, J.A. Hardy, Mechanism of zinc-mediated inhibition of caspase-9, Protein Science 21(7) (2012) 1056-1065.

[25] D.K. Perry, M.J. Smyth, H.R. Stennicke, G.S. Salvesen, P. Duriez, G.G. Poirier, Y.A. Hannun, Zinc is a potent inhibitor of the apoptotic protease, caspase-3 a novel target for zinc in the inhibition of apoptosis, Journal of Biological Chemistry 272(30) (1997) 18530-18533.

[26] H.R. Stennicke, G.S. Salvesen, Biochemical characteristics of caspases-3,-6,-7, and-8, Journal of Biological Chemistry 272(41) (1997) 25719-25723.

[27] M. Hamatake, K. Iguchi, K. Hirano, R. Ishida, Zinc induces mixed types of cell death, necrosis, and apoptosis, in molt-4 cells, The Journal of Biochemistry 128(6) (2000) 933-939.

[28] J. Mcaninly, D.L.H. Williams, S.C. Askew, A.R. Butler, C. Russell, Metal-Ion Catalysis in Nitrosothiol (Rsno) Decomposition, J Chem Soc Chem Comm (23) (1993) 1758-1759.

[29] D.L.H. Williams, The chemistry of S-nitrosothiols, Accounts Chem Res 32(10) (1999) 869876.

[30] A.P. Dicks, H.R. Swift, D.L.H. Williams, A.R. Butler, H.H. AlSadoni, B.G. Cox, Identification of $\mathrm{Cu}+$ as the effective reagent in nitric oxide formation from S-nitrosothiols (RSNO), J Chem Soc Perk T 2 (4) (1996) 481-487.

[31] C.W. McCarthy, R.J. Guillory, J. Goldman, M.C. Frost, Transition-Metal-Mediated Release of Nitric Oxide (NO) from S-Nitroso-N-acetyl-D-penicillamine (SNAP): Potential Applications for Endogenous Release of NO at the Surface of Stents Via Corrosion Products, Acs Appl Mater Inter 8(16) (2016) 10128-10135.

[32] M.W. Foster, J.R. Pawloski, D.J. Singel, J.S. Stamler, Role of circulating S-nitrosothiols in control of blood pressure, Hypertension 45(1) (2005) 15-17. 
[33] J.E. Freedman, R. Sauter, E.M. Battinelli, K. Ault, C. Knowles, P.L. Huang, J. Loscalzo, Deficient platelet-derived nitric oxide and enhanced hemostasis in mice lacking the NOSIII gene, Circ Res 84(12) (1999) 1416-1421.

[34] P. Kubes, M. Suzuki, D.N. Granger, Nitric-Oxide - an Endogenous Modulator of Leukocyte Adhesion, P Natl Acad Sci USA 88(11) (1991) 4651-4655.

[35] D.J. Lefer, S.P. Jones, W.G. Girod, A. Baines, M.B. Grisham, A.S. Cockrell, P.L. Huang, R. Scalia, Leukocyte-endothelial cell interactions in nitric oxide synthase-deficient mice, Am J Physiol-Heart C 276(6) (1999) H1943-H1950.

[36] U.C. Garg, A. Hassid, Nitric Oxide-Generating Vasodilators and 8-Bromo-Cyclic Guanosine-Monophosphate Inhibit Mitogenesis and Proliferation of Cultured Rat Vascular Smooth-Muscle Cells, J Clin Invest 83(5) (1989) 1774-1777.

[37] R.P. Singh, S. Kumar, R. Nada, R. Prasad, Evaluation of copper toxicity in isolated human peripheral blood mononuclear cells and it's attenuation by zinc: ex vivo, Molecular and cellular biochemistry 282(1-2) (2006) 13 .

[38] G.f. Hu, Copper stimulates proliferation of human endothelial cells under culture, Journal of cellular biochemistry 69(3) (1998) 326-335.

[39] A. Sakamoto, H. Jinnouchi, S. Torii, R. Virmani, A.V. Finn, Understanding the Impact of Stent and Scaffold Material and Strut Design on Coronary Artery Thrombosis from the Basic and Clinical Points of View, Bioengineering (Basel) 5(3) (2018).

[40] N. Pal, J. Din, P. O'Kane, Contemporary Management of Stent Failure: Part One, Interv Cardiol 14(1) (2019) 10-16.

[41] R.A. Byrne, M. Joner, A. Kastrati, Stent thrombosis and restenosis: what have we learned and where are we going? The Andreas Gruntzig Lecture ESC 2014, Eur Heart J 36(47) (2015) 3320-31.

[42] K. Katsanos, S. Spiliopoulos, P. Kitrou, M. Krokidis, D. Karnabatidis, Risk of Death Following Application of Paclitaxel-Coated Balloons and Stents in the Femoropopliteal Artery of the Leg: A Systematic Review and Meta-Analysis of Randomized Controlled Trials, J Am Heart Assoc 7(24) (2018) e011245.

[43] H. Jinnouchi, S. Torii, A. Sakamoto, F.D. Kolodgie, R. Virmani, A.V. Finn, Fully bioresorbable vascular scaffolds: lessons learned and future directions, Nat Rev Cardiol (16) (2018) 286-304. 


\section{Concluding Remarks}

This work illuminates the complex interplay between degrading zinc-based implant materials and the vascular environment. Current simplistic conceptualizations of the foreign body response must be reconsidered when examining degradable materials. The field of biomaterials has witnessed a fevered introduction of degradable materials over the past 20 years, including metals, polymers, composites, additively manufactured matrices, and natural substrates. With the addition of zinc as a new class of biomaterials, our understanding of how the innate and adaptive immune systems interact with implanted materials must evolve. In the body of evidence provided in chapter 1, we have shown that the inflammatory response is capable of accommodating degrading zinc materials. Identifying links between cellular driving forces that increase corrosion activity and vice versa could allow for formulating a theoretical foundation that further enhances our abilities to engineer advanced degradable biomaterials.

When designing zinc-based biomaterials for cardiovascular use, it is imperative to observe the material in the "application environment". Conventional 2-D cell culture methodology does not provide meaningful insight when applied to materials intended to operate in the complex environment of the intraluminal arterial space. Conversely, manufacturing of stents and implantation in pig models is prohibitively expensive. To bridge the gap, we developed a simple and effective methodology to accelerate the invention of novel degradable biomaterials and accomplish their screening based on their quantified performance in a realistic experimental rat model. Using this approach, presented in detail in chapter 2 , material characteristics in a wire implant geometry can be described in terms of their contribution to neointimal growth. To explore this approach, the author evaluated different surfaces with varied surface oxide layer stability engineered on zinc substrates, presented in chapter 3 . The reduction in biocompatibility for one of the surfaces was related to a relatively low surface oxide stability that increased susceptibility for pitting corrosion. We found that pitting corrosion vs. uniform corrosion leads to localized bursts of zinc corrosion, producing in turn a highly localized inflammatory reaction that worsens neointimal growth. This led us to conceptualize the 
engineering of zinc implants with a "tunable" neointimal response by controlling the surface oxide film stability. Taken together, the chapter illustrates how relating material characteristics to the micro cellular and macro tissue development provides fundamental insight into the neointimal responses, which in turn can impact the metallurgical engineering of the material.

In addition to exploring relationships between material properties and biological responses, the framework of testing that we developed in chapter 2 can be applied to the purpose of identifying the best performing materials fabricated by our research group. By statistical comparisons of biocompatibility metrics using high sample sizes, we have identified the quinary alloy $(\mathrm{Zn}-4 \mathrm{Ag}-0.8 \mathrm{Cu}-0.6 \mathrm{Mn}-0.15 \mathrm{Zr})$ as the best performing material, following solution treatment to maximize mechanical properties. This is the first alloyed zinc material that performs better than pure zinc. The identification of this alloy is described in detail in chapter 4 . The mechanisms by which this material generates outstanding biological responses is clarified in chapter 5 and summarized as follows:

$\mathrm{I}$ - the addition of $\mathrm{Cu}$ reduces inflammation and increases endothelilaization

II - the provocation of inflammation by $\mathrm{AgZn}_{3}$ precipitates is decreased by solution treatment

III - zinc and copper exert a synergistic effect to suppress neointimal growth

In chapter 5 , the author begins to clarify the mechanisms by which zinc based degradable materials may suppress neointimal growth. When the neointimal development around platinum and zinc wires was compared, there was a reduced smooth muscle cell presence and an increased cellular apoptosis in the zinc neointima. The author hypothesized that the release of $\mathrm{Zn}^{2+}$ from degrading zinc-based implants induces programmed cell death, or apoptosis, of smooth muscle cells. The role of $\mathrm{Zn}^{2+}$ was confirmed by exposing arterial smooth muscle cells to high concentrations of $\mathrm{Zn}^{2+}$, ex vivo, in order to isolate the effects of ionic zinc from variables related to implant materials. The activity of the executioner caspase (3) was found to be elevated in high zinc conditions. This controlled form of cell 
death is beneficial to a progressively developing neointima, as it would shift the deathproliferation balance to stabilize against the progressive growth that eventually occludes stented arteries. This is an extremely important discovery since the proliferation of smooth muscle cells represents the hallmark mode of failure for arterial stents.

In summation, the work begins with the development of a new methodology to qualitatively and quantitatively evaluate the in vivo biocompatibility of degradable implant metals. This methodology was used to relate material characteristics to biological responses, including surface oxide film character, alloying composition, and bulk processing conditions. In addition to generating insights into fundamental relationships between materials and biocompatibility, the approach can be used to rank materials in terms of biocompatibility. From this approach, beneficial alloying elements and processing conditions can be identified. Beneficial elements identified by these approaches can be further investigated for their therapeutic value in the form of transition metals, since all the elements in the implant will be released due to biocorrosive activity.

Future studies will need to explore important questions that were generated by the present work. The present work evaluates materials in a normal, healthy animal system. While necessary to clarify fundamental relationships between material characteristics and neointimal formation and begin to clarify mechanisms of suppression by ionic zinc, a diseased arterial environment is more representative of the clinical environment. In contrast to a healthy artery, the diseased atherosclerotic environment is pro-inflammatory, more complex and unique per individual, and lipid laden. The performance of degradable zinc materials will need to be evaluated in a dynamic and pro-inflammatory microenvironment. The most suitable modeling systems for arterial disease have been developed decades ago by molecular biologists, using transgenic mice. It should be possible to adapt the implant surgery and our quantitative morphometric approach to the mouse. Positive performance of degradable zinc-based materials in diseased arteries would increase confidence in the successful clinical translation of materials selected using the approaches described in the present dissertation. 


\section{A Appendix for Chapter 1}
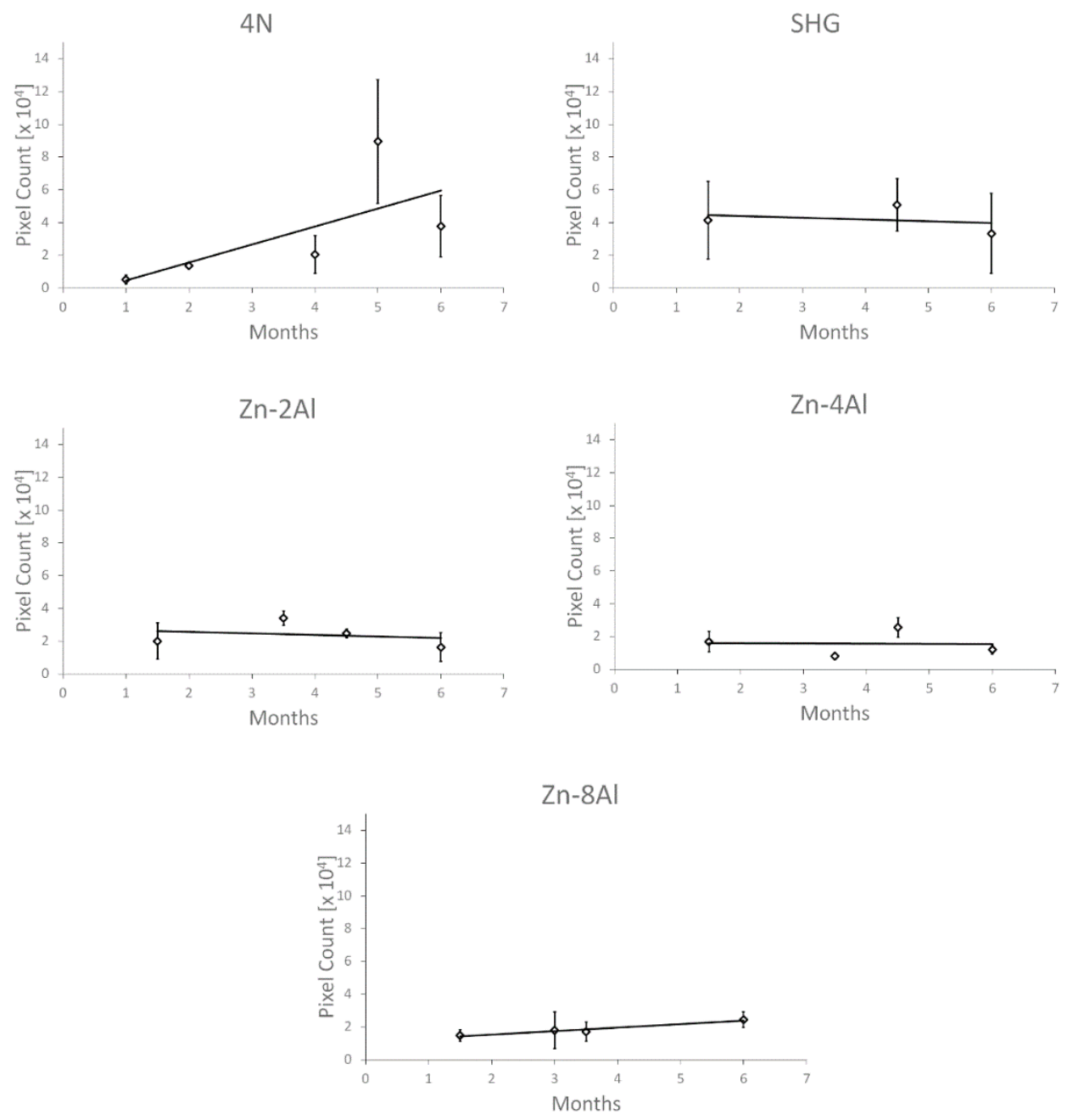

Fig. 1-S1. Quantification of CD163 staining using average pixel counting of multiple tissue sections per sample. 

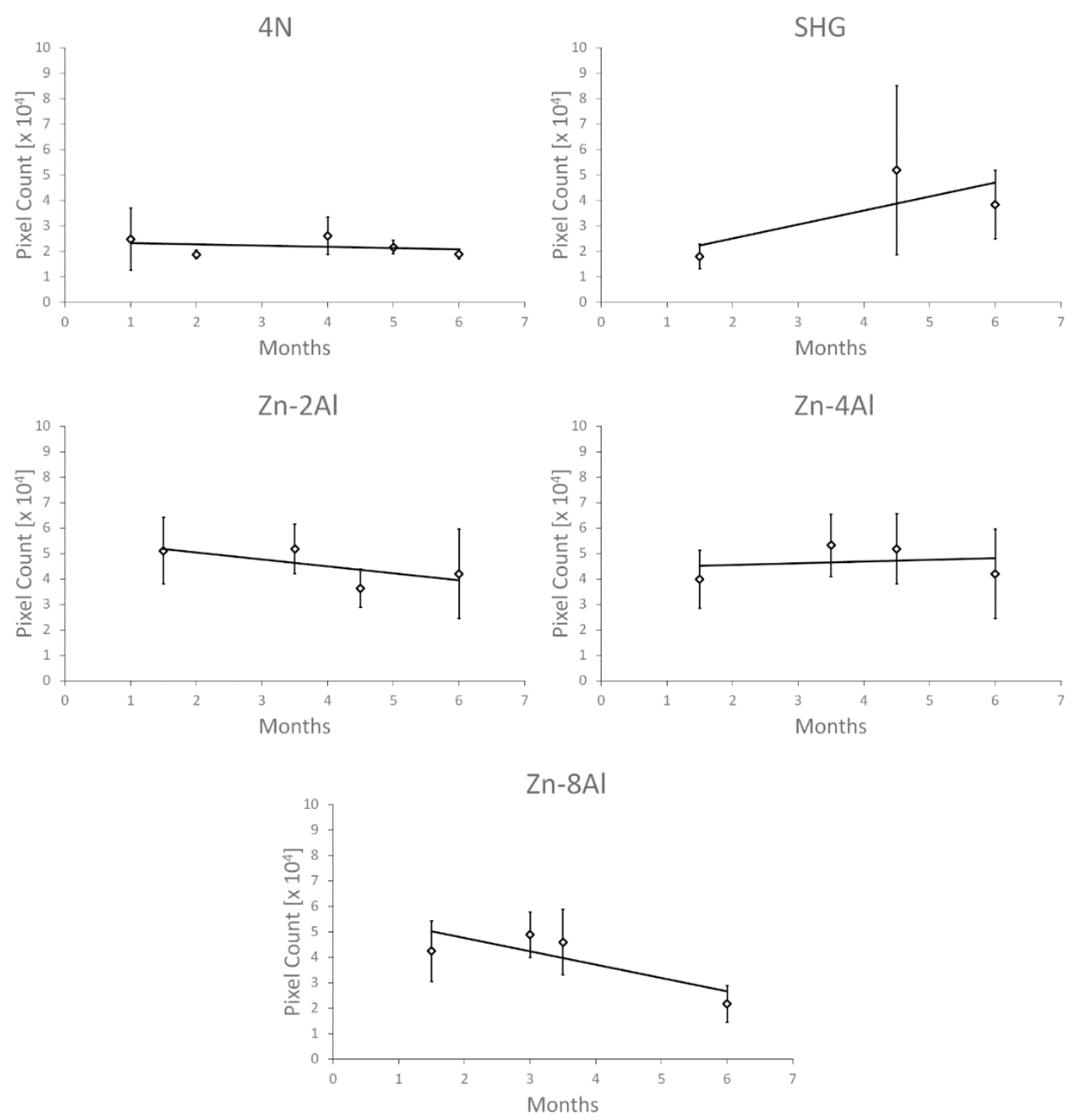

Fig. 1-S2. Quantification of $C D 11 b / c$ staining using average pixel counting of multiple tissue sections per sample. 

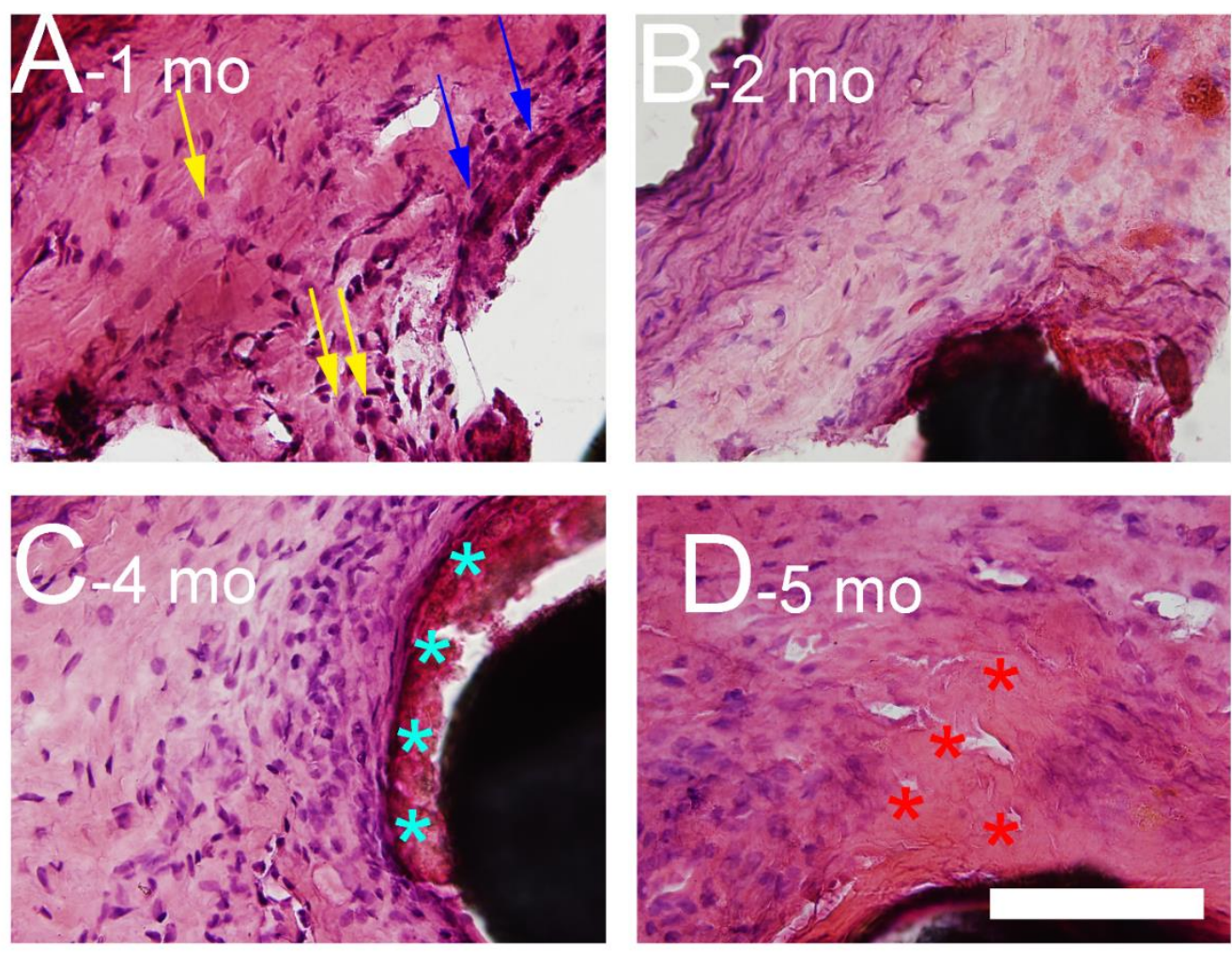

Fig. 1-S3. In depth clarification for Fig. 2. Yellow arrows in panel A show mononuclear cells. Cyan asterisk in $\mathrm{C}$ show region of compact corrosion product with no nuclear staining. Red asterisk in $D$ depict region of necrosis. Scale bar is set to $100 \mu \mathrm{m}$. 

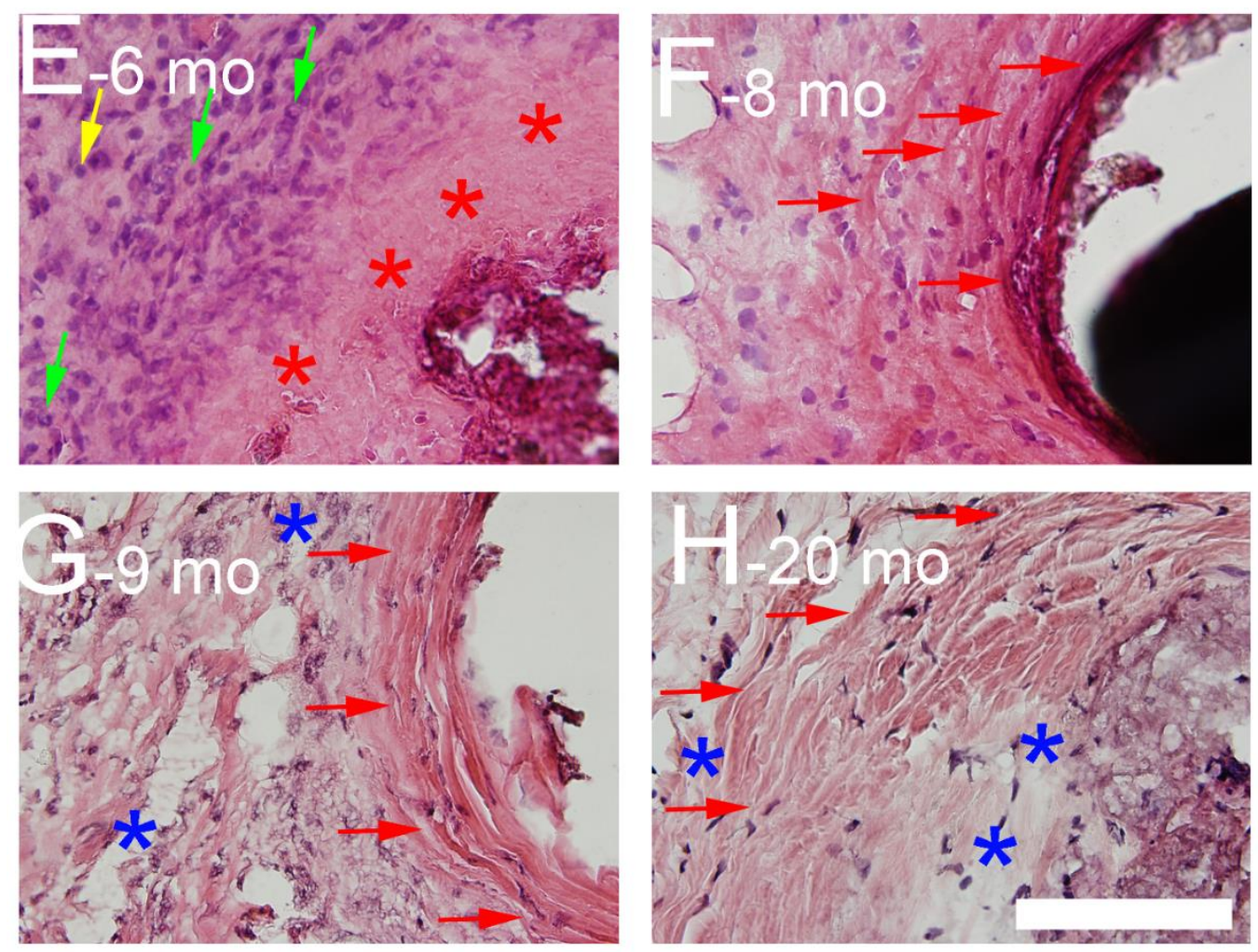

Fig. 1-S4. Second clarification of Fig. 2. Red asterisk in E show necrotic regions, yellow arrow displays a representative normal nuclear morphology, and the green arrows depict representative abnormal nuclear morphology. Red arrows in $F$ identify thick, circumferentially aligned collagenous fibers, while in panels $G$ and $\mathrm{H}$ show a distinct collagenous capsule. Blue asterisks in panel $\mathrm{G}$ identify chronic inflammation outside of the capsule, while in panel $\mathrm{H}$ they identify the chronic inflammation both inside and outside the capsule. Scale bar is set at $100 \mu \mathrm{m}$. 

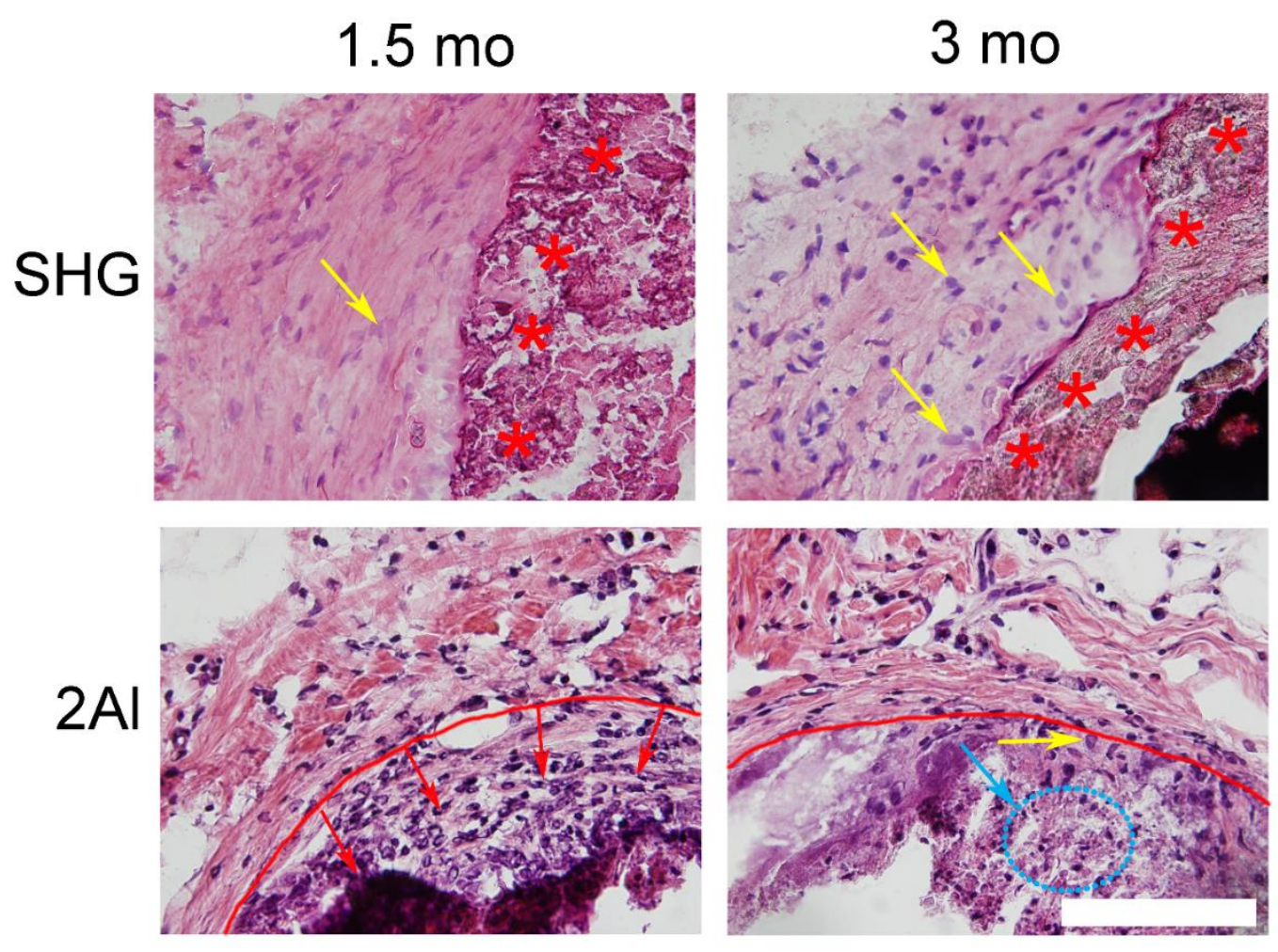

Fig. 1-S5. Red asterisks for the SHG explant at 1.5 and 3 months show large amounts of corrosion product. Yellow arrows show representative viable mononuclear cells. The red line for the bottom two panels depict the original border of corrosion pocket, with the red arrows showing the direction of cellular penetration. Light blue arrow in bottom right panel shows a representative region of pyknosis and karyorrhexis. Scale bar is at $100 \mu \mathrm{m}$ 

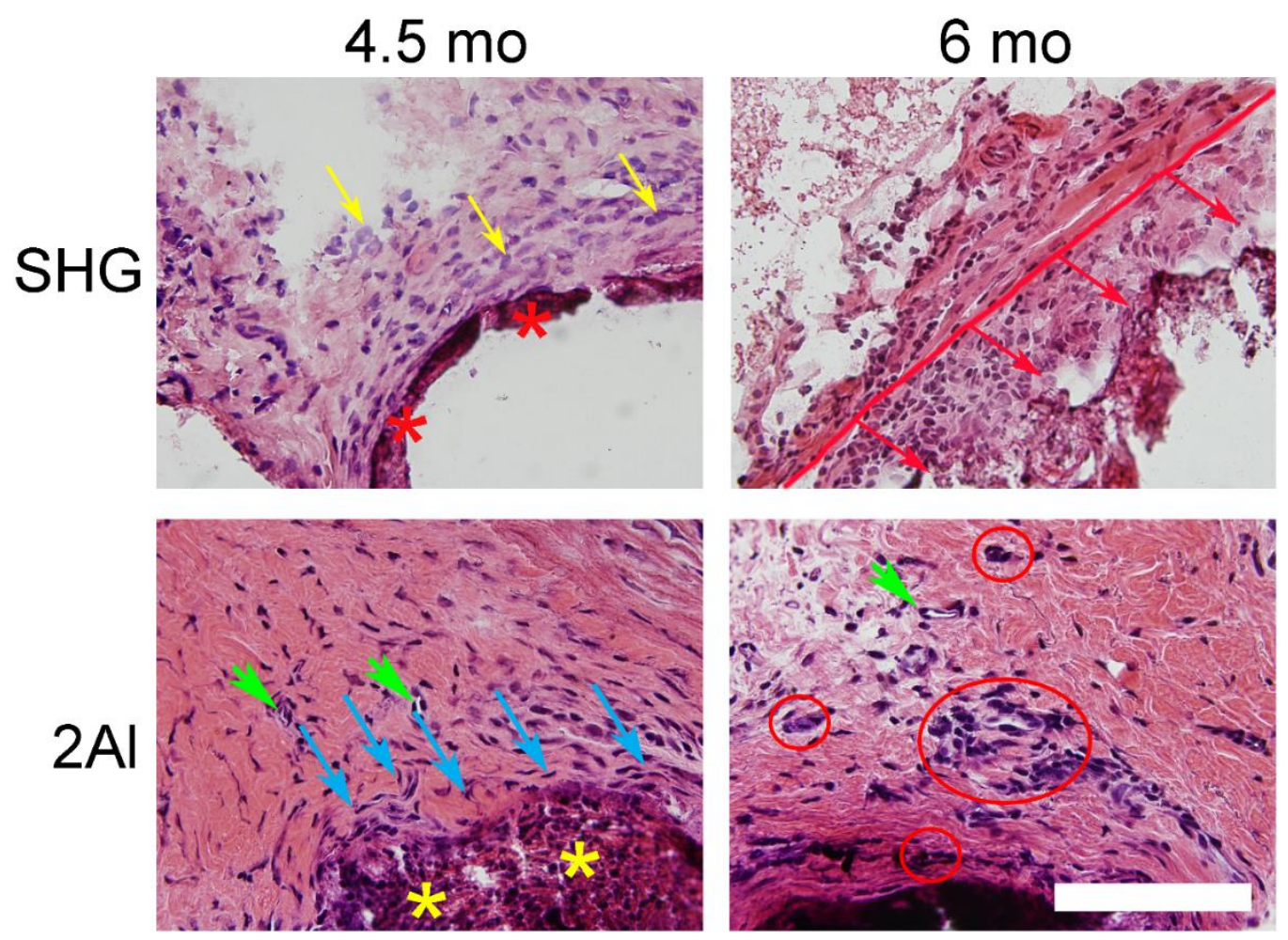

Fig. 1-S6. Yellow arrows in top left panel show representative mononuclear cells, while the red arrows in the top right panel portray the cellular movement from the original tissue - corrosion product border inwards towards the corroding implant. The green arrows in the bottom panels show neovascularization, and the cyan arrows identify representative fibroblasts. The yellow asterisks in the 2Al 4.5month panel shows corrosion product impregnated with cell nuclei. The red circles in the bottom right panel show sites of macrophage fusion. Scale bar is set at $100 \mu \mathrm{m}$. 

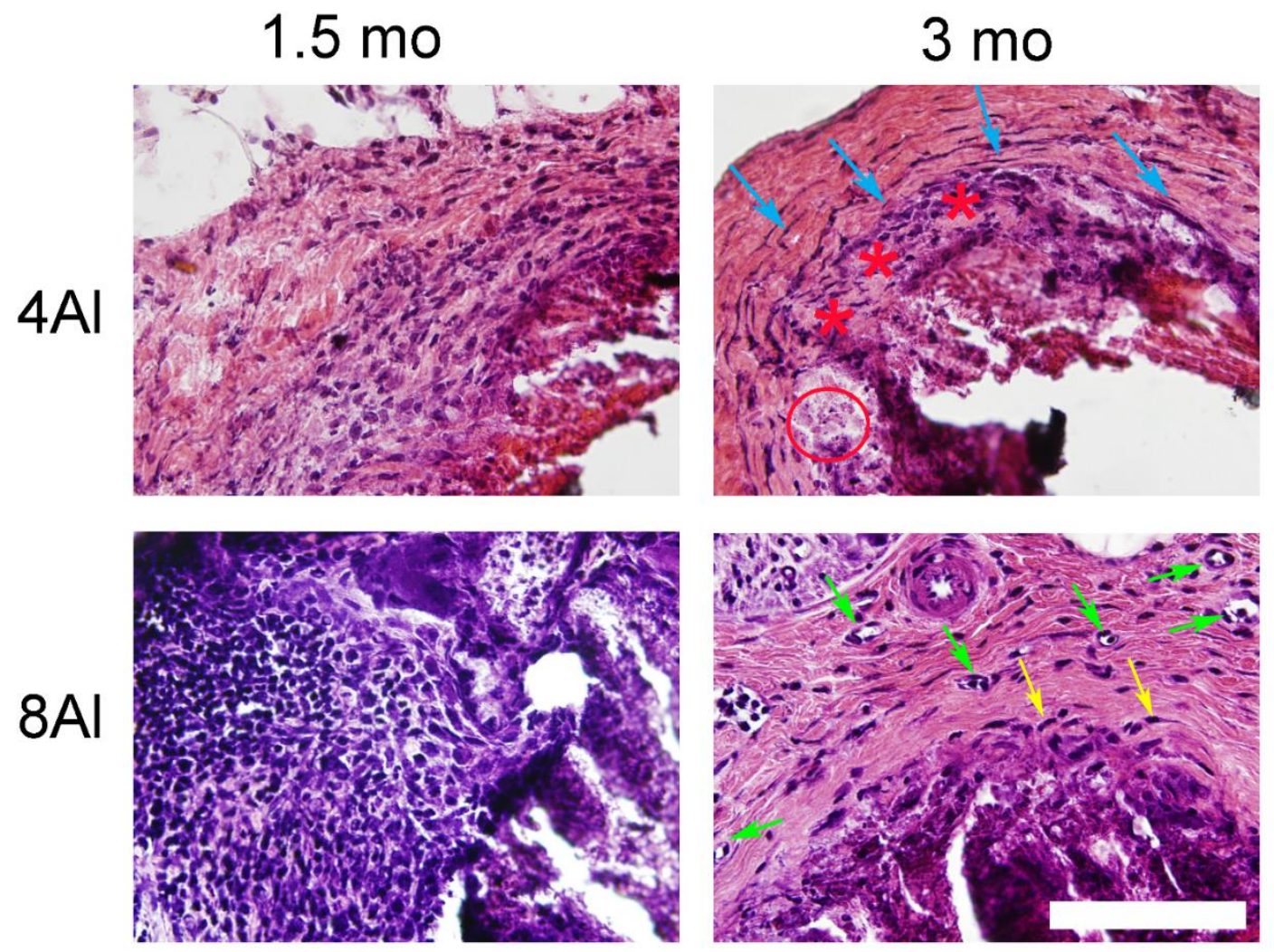

Fig. 1-S7. Cyan arrows in the 4-Al 3 month panel show representative mature fibroblasts at the interface. Red asterisks show representative inflammatory cells. Green arrows in the bottom right panel show neovascularization. Yellow arrows depict aligning fibroblasts. Scale bar is set at $100 \mu \mathrm{m}$. 

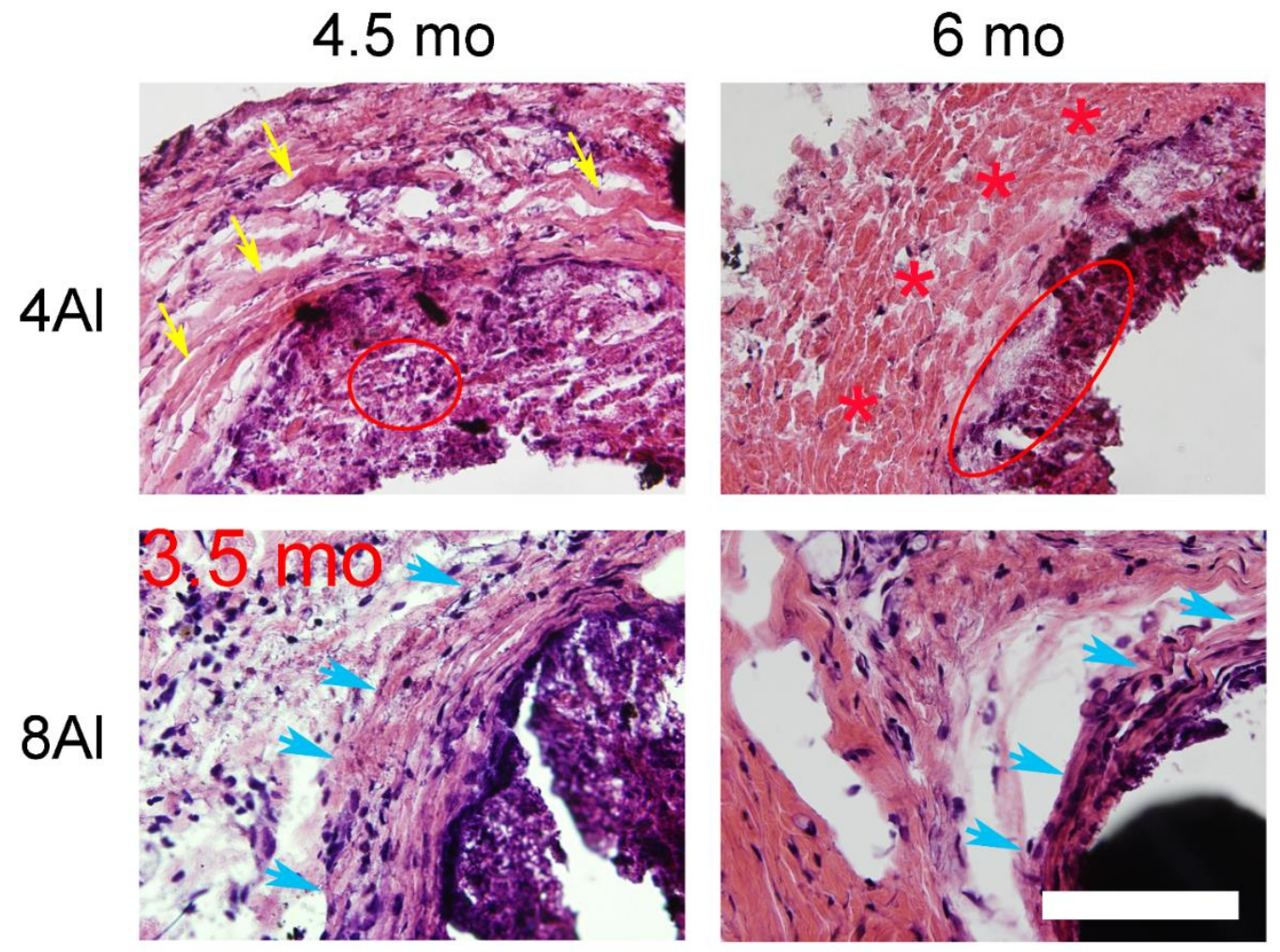

Fig. 1-S8. Yellow arrows in the top left panel show acellular collagenous bundles, with the red circle identifying representative dying inflammatory cells within the corrosion layer. The 4-Al 6 month panel's red asterisks show a thick, acellular collagenous capsule, with the red oval identifying inflammatory cells. The cyan arrows in the Zn-8Al 3.5 and 6 month panels identify the fibrous capsule. The scale bar is set at $100 \mu \mathrm{m}$. 

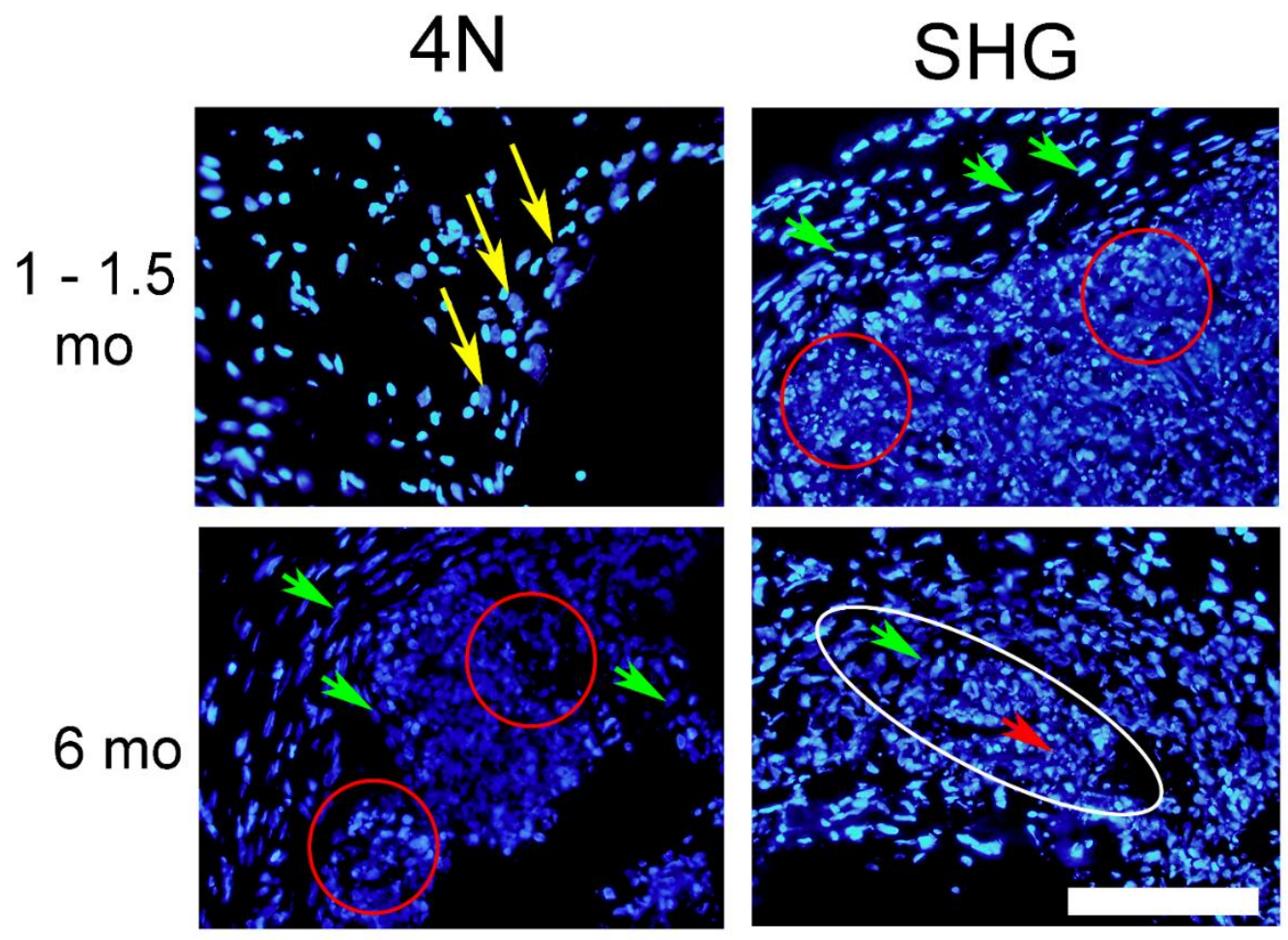

Fig. 1-S9. The top left panel's yellow arrows identify representative large mononuclear cells. In the 1.5 month SHG panel, the green arrows identify representative intact cells, while the red circles identify characteristic piknosis and karyorrhexis. The green arrows in the 6 month $4 \mathrm{~N}$ panel also show viable cells, while the red circles show areas of cell death. In the 6 month SHG panel, the white oval shows a region incorporating both viable and non-viable nuclei (green and red arrows respectively). Scale bar is set to $100 \mu \mathrm{m}$. 

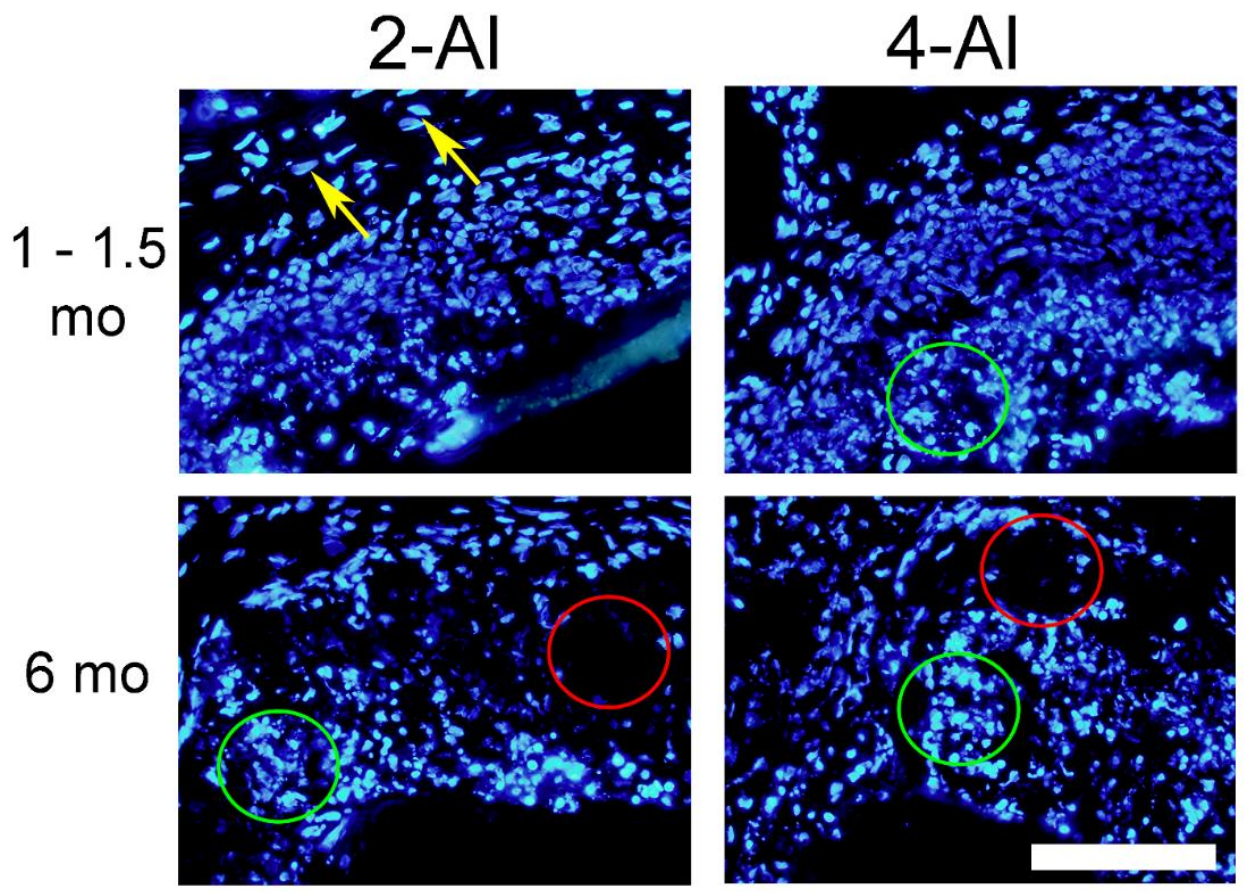

Fig. 1-S10. Yellow arrows in 1.5-month 2-Al panel identify cells of normal nuclear morphology. Green circles of all panels show highly irregular nucleic features with increased DAPI strength. Red circles show areas void of cell nuclei. Scale bar is set at $100 \mu \mathrm{m}$ 


\section{B Appendix for Chapter 3}

Table 3-S1. Fitting parameters of EP and AD samples after different immersion times

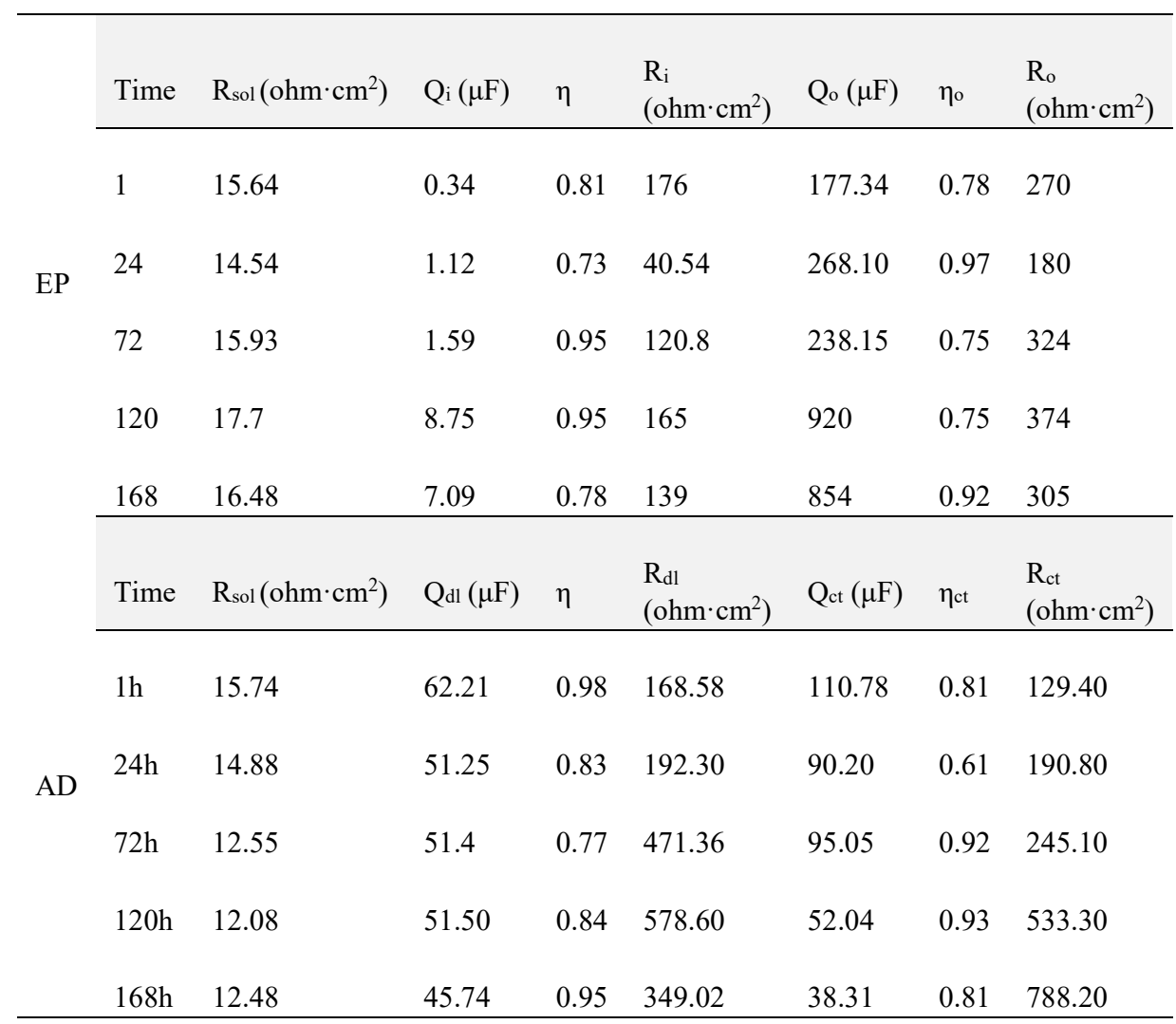




\section{Permission for Chapter 1}

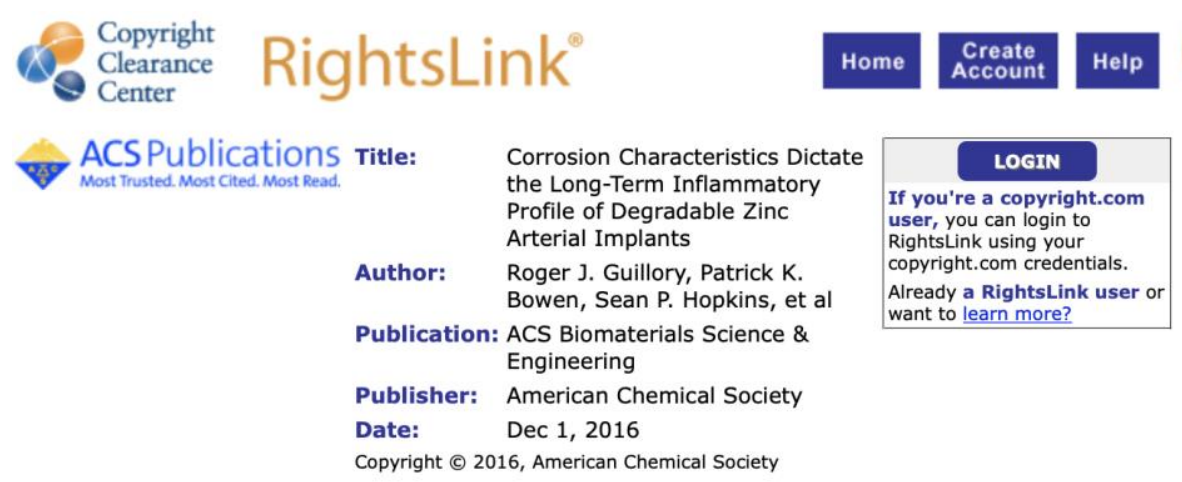

\section{PERMISSION/LICENSE IS GRANTED FOR YOUR ORDER AT NO CHARGE}

This type of permission/license, instead of the standard Terms \& Conditions, is sent to you because no fee is being charged for your order. Please note the following:

- Permission is granted for your request in both print and electronic formats, and translations.

- If figures and/or tables were requested, they may be adapted or used in part.

- Please print this page for your records and send a copy of it to your publisher/graduate school.

- Appropriate credit for the requested material should be given as follows: "Reprinted (adapted) with permission from (COMPLETE REFERENCE CITATION). Copyright (YEAR) American Chemical Society." Insert appropriate information in place of the capitalized words.

- One-time permission is granted only for the use specified in your request. No additional uses are granted (such as derivative works or other editions). For any other uses, please submit a new request. 


\section{D $\quad$ Permission for Chapter 2}

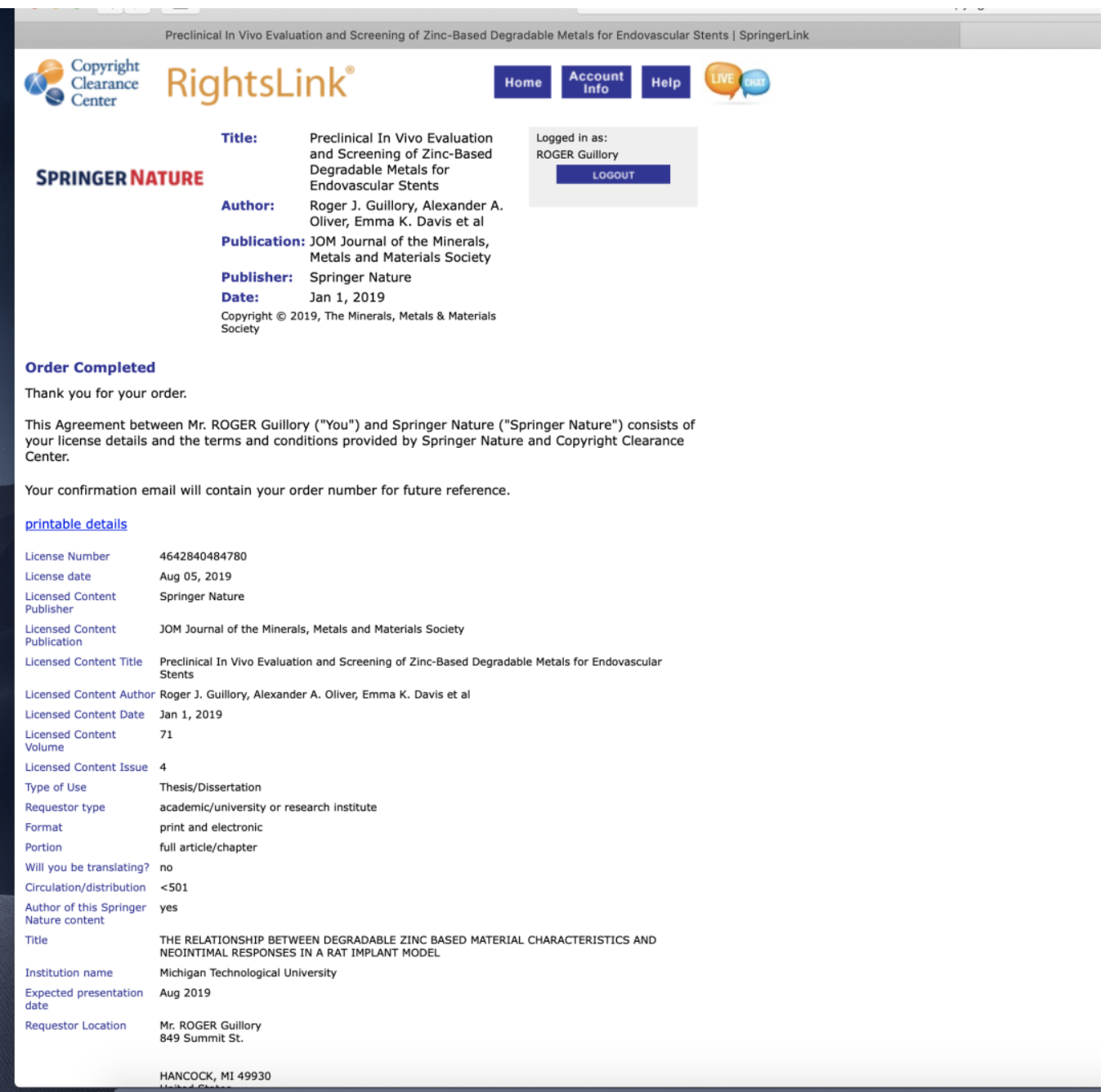




\section{E Permission for Chapter 3}

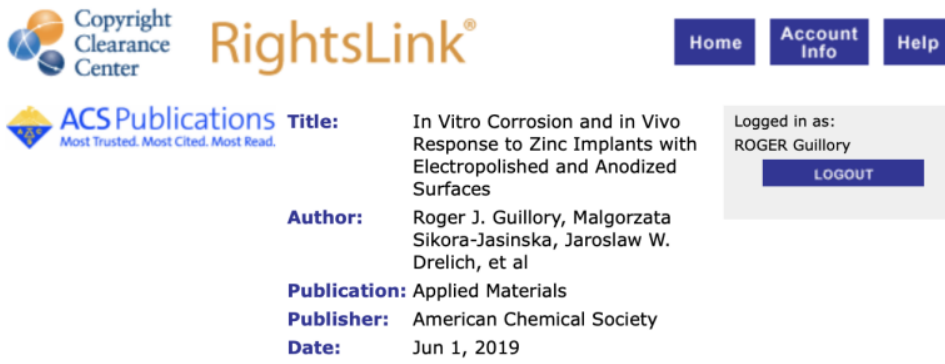

PERMISSION/LICENSE IS GRANTED FOR YOUR ORDER AT NO CHARGE

This type of permission/license, instead of the standard Terms \& Conditions, is sent to you because no fee is being charged for your order. Please note the following:

- Permission is granted for your request in both print and electronic formats, and translations.

- If figures and/or tables were requested, they may be adapted or used in part.

- Please print this page for your records and send a copy of it to your publisher/graduate school.

- Appropriate credit for the requested material should be given as follows: "Reprinted (adapted) with permission from (COMPLETE REFERENCE CITATION). Copyright (YEAR) American Chemical Society." Insert appropriate information in place of the capitalized words.

- One-time permission is granted only for the use specified in your request. No additional uses are granted (such as derivative works or other editions). For any other uses, please submit a new request. 